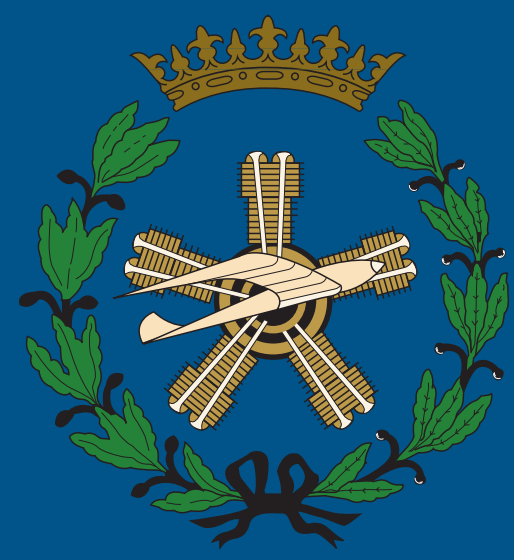

UNIVERSIDAD POLITÉCNICA DE MADRID

ESCUELA TÉCNICA SUPERIOR DE

INGENIEROS AERONÁUTICOS

\title{
OPTIMAL ENERGY HARVESTING FROM VORTEX-INDUCED AND TRANSVERSE GALLOPING VIBRATIONS
}

Doctoral Thesis

David Vicente Ludlam

Ingeniero Aeronáutico 
Departamento de Mecánica de Fluidos y Propulsión Aeroespacial

ESCUELA TÉCNICA SUPERIOR DE INGENIEROS AERONÁUTICOS

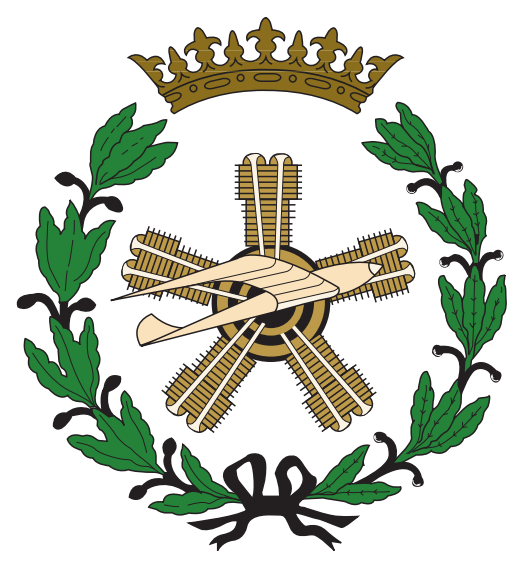

\title{
OPTIMAL ENERGY HARVESTING FROM VORTEX-INDUCED AND TRANSVERSE GALLOPING VIBRATIONS
}

Doctoral Thesis

\author{
Author: \\ David Vicente Ludlam \\ Ingeniero Aeronáutico
}

Directed by:

Antonio Barrero Gil

Doctor Ingeniero Industrial

2017 


\section{Acknowledgments}

First and foremost, I would like to express my sincere gratitude to my thesis director Professor Antonio Barrero Gil for the continuous support provided during the research, for his patience, motivation, and immense knowledge. His guidance helped me in all the time of research and writing of this thesis. I could not have imagined having a better director and mentor for my Ph.D study. Apart from my director, I would like to thank the rest of the members of the research group including Professor Ángel Velázquez López and Profesor Juan Ramón Arias Pérez for their insightful comments and relentless support.

My sincere thanks also goes to Miguel de las Heras, Ricardo de las Heras and José Sánchez for their continuous support in the laboratory, which made such outstanding experimental facilities possible. They would always surpass the desired quality of the experimental hardware required. I would also like to thank my fellow labmates, Miguel Reyes Mata and Francisco Sastre Matesanz with whom I have spent so many years and Ji Xu Xu who was always willing to give me a hand whenever I needed.

This research has been funded by the international company REPSOL S.A., through the REPSOLINSPIRE program and under contract P120130288. Through this program I was able to personally meet great professionals and people including Bernardo del Amo Fernández and Oscar Prieto Acedo.

Last but not least, I would like to thank my parents Enrique and Gloria for their encouragement and support throughout my life. I would not have been able to reach my goals without them. Also my girlfriend Paula for her understanding, endless patience and encouragement when it was most needed. 


\section{Abstract}

The increase in awareness of the negative impact of fossil fuels on the environment has been the main driver behind the development of renewable energy technologies in the last few years. Renewable energy sources are well suited for a distributed generation concept, in which generation and storage of energy is located close to the final load with low power capacities (usually $10 \mathrm{MW}$ or less), which enables the use of different sources leading to lower environmental impact. Some of the main renewable sources typically used include biomass, biogas, solar power, geothermal power and geophysical flows power. In particular, geophysical flows constitute an immense natural reservoir of energy present worldwide, being present as wind, river, oceanic or tidal flows among others. Most of the concepts operating nowadays that take advantage of fluid flows to harness energy rely on horizontal-axis turbines. However, conventional wind or water turbines cannot provide efficient power conversion to low-power applications, thus encouraging to seek new technologies to extract energy from fluid flows which can present big differences in their characteristics (namely density, mean velocity, turbulence intensity, etc.) efficiently.

Flow-Induced Vibrations (FIV) arise as a promising way of harvesting energy from such geophysical flows efficiently. Coupling phenomena between the dynamics of flexibly-mounted structures and the surrounding flows can lead to self-sustained oscillations of a solid body which correspondingly can be transformed into electrical energy by means of a transducer. Many fluid-solid interactions have been considered recently as a mean to extract energy from current flows including Vortex-Induced Vibrations (VIV), transverse or torsional galloping, flutter or wake-induced vibrations among others. In the present thesis, VIV and transverse galloping oscillations are investigated and optimal configurations will be sought so as to maximize energy production.

In first place, VIV of a circular cylinder is investigated. VIV is a resonant type of FIV where large-scale vortices are shed periodically from the body surface which leads to an alternating fluid force on the body. As such, oscillations only occur for a given range of inflow velocities where vortex emission frequency coincides with the natural frequency of oscillation of the body. In particular, it is investigated if through an active rotation of the circular cylinder along its axis proportional to the dynamics of the cylinder (namely, proportional to its transverse displacement or its transverse velocity) it is possible to increase the maximum amplitude of oscillation of the cylinder as well as increasing the range of velocities of the incident flow that yield large amplitude oscillations (broadband character) with the purpose of enhancing energy extraction. Also, as a side result the possibility of reducing oscillations in order to protect structures not meant to suffer from oscillations is evaluated. The effect of rotating the circular cylinder undergoing FIV oscillations is investigated both numerically for the low end Reynolds regime (through a Lattice Boltzmann Method) and experimentally at the "Antonio Barrero Ripoll" free-surface recirculating water channel for the medium range of Reynolds number. Additionally, Particle Image Velocimetry (PIV) technique has been used to visualize the main wake structures appearing behind the rotating cylinder in order to qualitatively determine the effect such rotation has on the vortical structures appearing. 
Secondly, energy harvesting from transverse galloping is studied. Transverse galloping is a selfinduced instability, in which a small displacement of the body immersed in a cross-flow leads to an oscillatory motion with increasing amplitude once a critical velocity is surpassed. Differently from VIV, transverse galloping is not a resonant type of FIV, thus, once transverse galloping shoots, amplitude continues growing with the incident velocity. Generally speaking, it has been proven that transverse galloping can be correctly described through a non-linear quasi-steady description of the fluid forces as a result of the disparity between the characteristic timescale of motion of the body and the convective timescale. This allows to analytically treat the problem. Here, an integral analytical treatment of the energy harvesting problem through transverse galloping is investigated. In particular, two types of transducers are considered (electromagnetic/piezoelectric) in order to obtain optimal configurations. Also a dual-mass system is investigated. A secondary (out of the flow) mass is elastically mounted to the galloping prism to determine if efficiency can be enhanced. Finally, the possibility of rotating the galloping body is considered to increase energy extraction through a quasi-steady model and compared to numerical simulations of a D-section cross-section at Reynolds 100. 


\section{Resumen}

La creciente conciencia sobre los efectos negativos de los combustibles fósiles en el medio ambiente se ha convertido en el principal motor de desarrollo de tecnologías asociadas a la generación de energías renovables en los últimos años. Así mismo, dichas fuentes se han adecuado paulatinamente al concepto de generación distribuida, en el que la generación y almacenamiento de dicha energía se ubica cerca del destinatario final con capacidades de baja potencia (usualmente $10 \mathrm{MW}$ o menos), lo que permite utilizar diferentes fuentes que conduzcan a un menor impacto ambiental. Algunas de las principales fuentes renovables que se usan típicamente incluyen la biomasa, biogás, energía solar, energía geotérmica y energía a partir de corrientes geofísicas. Estas corrientes constituyen un inmenso reservorio natural de energía que se encuentra presente en todo el planeta, pudiendo aparecer como corrientes de viento, ríos, corrientes en el océano o de marea entre otros. La mayoría de los conceptos que operan hoy en día y que usan las corrientes geofísicas para extraer energía, se basan en turbinas de eje horizontal. Sin embargo, los aerogeneradores o turbinas de agua convencionales no pueden proporcionar una conversión de energía eficiente para aplicaciones de baja potencia. Esta limitación ha fomentado la búsqueda de nuevas tecnologías que puedan extraer energía de corrientes con grandes diferencias en sus características (densidad, velocidad media, intensidad de turbulencia, etc.) de manera eficiente.

Las vibraciones inducidas por flujo (FIV por sus siglas en inglés) surgen como un método prometedor para extraer energía de dichas corrientes geofísicos eficientemente. Los fenómenos de acoplamiento entre las estructuras elásticas y las corrientes circundantes pueden conducir a oscilaciones autosostenidas del cuerpo, que a su vez y de manera apropiada, se pueden transformar en energía eléctrica mediante el empleo de un transductor. Recientemente se ha considerado la posibilidad de utilizar diversos fenómenos de interacción fluido-sólida para extraer energía a partir de corrientes geofísicas, incluyendo entre otros fenómenos las vibraciones inducidas por vórtices (VIV por sus siglas en inglés), galope transversal o torsional, flameo o vibraciones inducidas por la estela. En la presente tesis se analizan las oscilaciones a partir de VIV y galope transversal para la búsqueda de configuraciones óptimas con el objetivo de maximizar la producción de energía.

En primer lugar, se explora el fenómeno de VIV de un cilindro circular. VIV es un tipo de resonancia dentro de los FIV, donde los vórtices de gran escala se desprenden periódicamente de la superficie del cuerpo que conduce a la aparición de una fuerza fluida alterna sobre el cuerpo. Como tal, las oscilaciones sólo ocurren para un rango dado de velocidades incidente de la corriente, en donde la frecuencia de emisión de vórtices coincide con la frecuencia natural de oscilación del cuerpo. En particular, se busca evidenciar si mediante una rotación activa del cilindro circular a lo largo de su eje, proporcional a la dinámica de dicho cilindro (es decir, proporcional a su desplazamiento transversal o a su velocidad transversal) es posible aumentar tanto la amplitud máxima de oscilación del cilindro, así como el rango de velocidades incidente de la corriente donde ocurre dicha resonancia, con el objeto de mejorar al máximo posible la extracción de energía. Además, como resultado secundario, se evalúa la posibilidad de reducir las oscilaciones para proteger posibles estructuras que no estén destinadas a oscilar. El efecto de la rotación del cilindro circular sometido a oscilaciones de FIV se investiga 
tanto numéricamente para el régimen de bajo número de Reynolds (mediante un esquema de Lattice Boltzmann Method), como experimentalmente en el canal de agua de recirculación de superficie libre "Antonio Barrero Ripoll" para el rango medio del número de Reynolds. Adicionalmente, se ha utilizado la técnica de Velocimetría de Imagen de Partículas (PIV) para visualizar las estructuras principales de la estela que aparecen tras el cilindro y determinar cualitativamente el efecto que tiene dicha rotación sobre los vórtices que aparecen.

En segundo lugar, se examina la generación de energía a partir del galope transversal. El galope transversal es una inestabilidad autoinducida, en la que un pequeño desplazamiento del cuerpo inmerso en un flujo transversal conduce a un movimiento oscilatorio con amplitud creciente, una vez superada una velocidad crítica. A diferencia de VIV, el galope transversal no es un tipo de resonancia dentro de los FIV, por lo tanto, una vez que aparece el galope transversal, la amplitud continúa aumentando con la velocidad incidente. En general, se ha demostrado que el galope transversal se puede describir correctamente a través de una descripción cuasi-estática no lineal de las fuerzas fluidas, como resultado de la disparidad entre la escala del tiempo característico de oscilación del cuerpo y la escala de tiempo de residencia fluida. Esto permite tratar analíticamente el problema. En la presente tesis se estudia un tratamiento analítico integral del problema de extracción de energía a partir del fenómeno de galope transversal. En un primer momento, se han considerado dos tipos de transductores (electromagnéticos / piezoeléctricos) para la obtención de configuraciones óptimas que maximicen la extracción energética. También se analiza un sistema de doble masa. Una masa secundaria (fuera del flujo) se monta elásticamente en el prisma galopante para determinar si se puede mejorar la eficiencia. Finalmente, se considera la posibilidad de girar el cuerpo galopante con el objeto de aumentar la extracción de energía analizándose a través de un modelo cuasi-estático y comparándolo con simulaciones numéricas a Reynolds 100. 


\section{Contents}

$\begin{array}{lc}\text { Acknowledgments } & \text { i }\end{array}$

$\begin{array}{ll}\text { Abstract } & \text { iii }\end{array}$

$\begin{array}{ll}\text { Resumen } & \mathbf{V}\end{array}$

1 Introduction $\quad 1$

1.1 Introduction . . . . . . . . . . . . . . . . . . . . . . 1

1.2 A new paradigm for flow induced vibrations . . . . . . . . . . . . . . . . . 2

1.2.1 Considerations on energy harvesting via FIV . . . . . . . . . . . . . . 4

1.3 VIV and transverse galloping . . . . . . . . . . . . . . . . . . . 6

1.3.1 Parameters governing VIV and transverse galloping . . . . . . . . . . . . . 7

$1.3 .2 \mathrm{VIV} \ldots \ldots \ldots \ldots \ldots \ldots$

1.3 .3 Transverse galloping . . . . . . . . . . . . . . . . 10

1.4 Objectives, outline and main results of the thesis . . . . . . . . . . . . . . 11

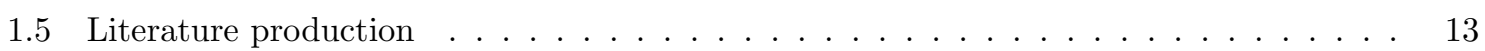

2 Numerical Flow-Induced Vibration of a rotating circular cylinder using position and velocity feedback 15

2.1 Introduction . . . . . . . . . . . . . . . . . . . . . . . 15

2.2 Numerical Method . . . . . . . . . . . . . . . . . . . . . 17

2.2 .1 Fluid-structure model . . . . . . . . . . . . . . . . . 17

2.2 .2 Numerical simulation method . . . . . . . . . . . . . . . . 18

2.2.3 Computational domain and boundary conditions . . . . . . . . . . . . . 19

2.2 .4 Force evaluation . . . . . . . . . . . . . . . . . . . 21

2.2 .5 Model validation . . . . . . . . . . . . . . . . . . 21

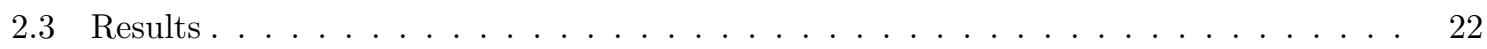

2.3.1 Rotation proportional to cylinder's displacement $\left(\theta=\tilde{k}_{1} Y\right) \ldots \ldots 23$

2.3.2 Rotation proportional to the cylinder's velocity $\left(\theta=\tilde{k}_{2} Y^{\prime}\right) \ldots \ldots$

2.4 Quasi-steady analysis . . . . . . . . . . . . . . . . . . . 36

2.5 Discussion and comparison between the two rotating laws . . . . . . . . . . . 38

2.5.1 Maximum angle of rotation . . . . . . . . . . . . . . . 38

2.5.2 On the intensity of the vortices shed . . . . . . . . . . . . 38

2.6 Concluding remarks . . . . . . . . . . . . . . . . . . . . . 39

3 Experimental investigation of flow-induced vibrations of a rotating circular cylinder using position and velocity feedback $\quad 43$

3.1 Introduction . . . . . . . . . . . . . . . . . . . . . . . 43

3.1.1 Differences in the VIV response emerging as a result of the Reynolds number . 44

3.2 Experimental setup and validation . . . . . . . . . . . . . . . . . 46 
3.2.1 Experimental setup . . . . . . . . . . . . . . . . 46

3.2 .2 Data reduction and rotation laws evaluated . . . . . . . . . . . . . 50

3.2 .3 PIV measurements . . . . . . . . . . . . . . . . . . . . 51

3.2 .4 Validation test . . . . . . . . . . . . . . . . . . . 54

3.3 Results . . . . . . . . . . . . . . . . . . . . . . . . . 54

3.3.1 Rotation proportional to position . . . . . . . . . . . . . 55

3.3.2 Rotation proportional to velocity . . . . . . . . . . . . . . . . 62

3.4 Concluding remarks . . . . . . . . . . . . . . . . . . . . . . . . 64

4 Optimal energy harvesting from transverse galloping $\quad 69$

4.1 Introduction . . . . . . . . . . . . . . . . . . . . 69

4.1.1 Brief introduction of the galloping phenomenon . . . . . . . . . . . . . . . 69

4.1.2 Galloping as a mechanism for energy harvesting . . . . . . . . . . . . . . . 72

4.2 Formulation of the problem . . . . . . . . . . . . . . . . . . . . . 74

4.2 .1 Mathematical model . . . . . . . . . . . . . . . . . . . . 74

4.2 .2 Transverse fluid force coefficient $C_{y} \ldots \ldots \ldots \ldots$. . . . . . . . 75

4.2.3 Analytical approximation . . . . . . . . . . . . . . . . . 75

4.2 .4 Energy harvesting efficiency . . . . . . . . . . . . . . . . . . 77

4.3 Optimal electromagnetic energy extraction . . . . . . . . . . . . . . . . . . . 79

4.3.1 Mathematical model . . . . . . . . . . . . . . . . . . . . . . . . 79

4.3.2 Galloping response, energy efficiency, and optimal electrical load resistance . . 80

4.3 .3 Concluding remarks . . . . . . . . . . . . . . . . . 88

4.4 Optimal piezoelectric energy extraction . . . . . . . . . . . . . . . . . . 89

4.4 Mathematical model . . . . . . . . . . . . . . . . . . . 89

4.4 .2 Galloping response and energy efficiency . . . . . . . . . . . . . . . . . 92

4.4.3 Role of $U^{*}$ and $\beta$ on the efficiency of the piezoelectric power harvester. . . . . 93

4.4 .4 Model validation with experimental results . . . . . . . . . . . . . 96

4.4 .5 Practical design application . . . . . . . . . . . . . . . . . . 96

4.4 .6 Concluding remarks . . . . . . . . . . . . . . . . . 98

4.5 Enhancing mechanical energy extraction using a DM system . . . . . . . . . . . . . 100

4.5.1 Mathematical model study for TG using a dual mass system for configuration C1100

4.5.2 Mathematical model study for TG using a dual mass system for configuration

$\mathrm{C} 2$ and $\mathrm{C} 3 \ldots \ldots \ldots \ldots \ldots \ldots \ldots$

4.5.3 Concluding remarks . . . . . . . . . . . . . . . . . . . . . 112

4.6 Enhancing energy extraction by rotating galloping body . . . . . . . . . . . . . . . . . 114

4.6 .1 Mathematical model . . . . . . . . . . . . . . . . . . . . . . 114

4.6.2 Rotation of the prism proportional to the angle of attack $\alpha_{F} \ldots \ldots$. . . . . 116

4.6.3 Rotation of the prism proportional to the displacement $\theta=K_{2} Y / U^{*} \ldots$. . . 122

4.6.4 Concluding remarks ......................... 125

5 Conclusions and future research $\quad 127$

$\begin{array}{lr}\text { A Effect of cantilevered beams } & 131\end{array}$

Bibliography 


\section{List of Figures}

1.1 Simplified schematics of the energy harvesting process from FIV . . . . . . . . . . 4

1.2 Elastically-mounted body free to oscillate tranversely to the cross-flow. . . . . . . . . . 7

1.3 (a) Amplitude response and (b) frequency response from VIV oscillations at $m^{*}=$ 2.2 from Nemes et al. (2012). Superimposed non-dimensional vorticity contours for different wake patterns from Morse and Williamson (2009). . . . . . . . . . . .

1.4 (a) Averaged transverse aerodynamic force coefficient, normal to the incident current for a square section prism measured in conditions of low turbulence of the incident current. (b) Transverse galloping response (LCO) of the same prism of square section with different values of mass and damping in a low level turbulence stream. (Parkinson and Smith, 1964). . . . . . . . . . . . . . . . .

2.1 Schematics of the rotating spring-mounted circular cylinder. . . . . . . . . . . . . 18

2.2 Schematics of the computational domain and boundary conditions. . . . . . . . . . . 20

2.3 (a) D2Q9 lattice model and (b) schematics of the moving boundary condition treatment of the circular cylinder. . . . . . . . . . . . . . . . . .

2.4 Comparison of a VIV amplitude curve for $m^{*}=12.7, \zeta=0$ at $\operatorname{Re}=100$ for Shiels et al. (2001) and the present study. . . . . . . . . . . . . . .

2.5 (a) In-line and (b) transverse mean force coefficients for a fixed rotating cylinder as a function of the rotation rate at $\mathrm{Re}=100$. Results from Bourguet and Jacono (2014) are used as reference. . . . . . . . . . . . . . . . . . .

2.6 (a) Maximum non-dimensional amplitude oscillation as a function of the reduced velocity for positive values of the rotating parameter $\tilde{k}_{1}$ and (b) for negative values of

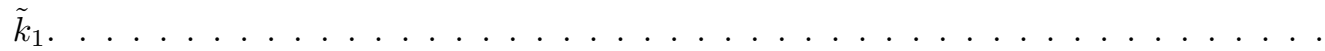

2.7 (a) Oscillating frequency of the cylinder as a function of the reduced velocity for positive values of the rotating parameter $\tilde{k}_{1}$ and (b) for negative values of $\tilde{k}_{1}$. Strouhal law is shown in bold line. . . . . . . . . . . . . . . . . . . . . . . . . .

2.8 (a) Non-dimensional amplitude of oscillation and (b) vorticity field at four different values of the reduced velocity for a rotating parameter of $\tilde{k}_{1}=0 . \ldots$. . . . . .

2.9 (a) Normalized frequency of oscillation $f^{*}$ (dashed line) and normalized frequency of vortex emission $f_{v}^{*}$ (solid line), (b) total transverse force coefficient $C_{y}$ (bold solid line), potential force coefficient $C_{p}$ (dashed line) and vortex force coefficient $C_{v}$ (solid line) and (c) FFT of the normalized vortex force coefficient $C_{v}$ at four values of the reduced velocity for a rotating parameter of $\tilde{k}_{1}=0 \ldots \ldots \ldots \ldots \ldots$

2.10 (a) Non-dimensional amplitude of oscillation and (b) vorticity field at four different values of the reduced velocity for a rotating parameter of $\tilde{k}_{1}=1.0 . \ldots$. . . . . 
2.11 (a) Normalized frequency of oscillation $f^{*}$ (dashed line) and normalized frequency of vortex emission $f_{v}^{*}$ (solid line), (b) total transverse force coefficient $C_{y}$ (bold solid line), potential force coefficient $C_{p}$ (dashed line) and vortex force coefficient $C_{v}$ (solid line) and (c) FFT of the normalized vortex force coefficient $C_{v}$ at four values of the reduced velocity for a rotating parameter of $\tilde{k}_{1}=1.0 \ldots \ldots \ldots \ldots$

2.12 (a) Non-dimensional amplitude of oscillation and (b) vorticity field at four different values of the reduced velocity for a rotating parameter of $\tilde{k}_{1}=-0.5$. . . . . . . . .

2.13 (a) Normalized frequency of oscillation $f^{*}$ (dashed line) and normalized frequency of vortex emission $f_{v}^{*}$ (solid line), (b) total transverse force coefficient $C_{y}$ (bold solid line), potential force coefficient $C_{p}$ (dashed line) and vortex force coefficient $C_{v}$ (solid line) and (c) FFT of the normalized vortex force coefficient $C_{v}$ at four values of the reduced velocity for a rotating parameter of $\tilde{k}_{1}=-0.5 \ldots \ldots \ldots \ldots$. . . . . . . .

2.14 (a) Non-dimensional amplitude of oscillation and (b) vorticity field at four different values of the reduced velocity for a rotating parameter of $\tilde{k}_{1}=-1.2 . \ldots . .$. .

2.15 (a) Normalized frequency of oscillation $f^{*}$ (dashed line) and normalized frequency of vortex emission $f_{v}^{*}$ (solid line), (b) total transverse force coefficient $C_{y}$ (bold solid line), potential force coefficient $C_{p}$ (dashed line) and vortex force coefficient $C_{v}$ (solid line) and (c) FFT of the normalized vortex force coefficient $C_{v}$ at four values of the reduced velocity for a rotating parameter of $\tilde{k}_{1}=-1.2 \ldots \ldots \ldots \ldots . . . \ldots$

2.16 (a) Maximum non-dimensional amplitude oscillation as a function of the reduced velocity for positive values of the rotating parameter $\tilde{k}_{2}$ and (b) for negative values of

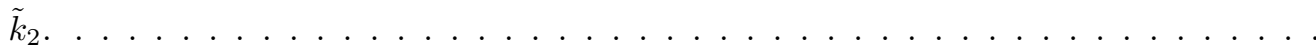

2.17 (a) Oscillating frequency of the cylinder as a function of the reduced velocity for positive values of the rotating parameter $\tilde{k}_{2}$ and (b) for negative values of $\tilde{k}_{2}$. Strouhal law is shown in bold line. . . . . . . . . . . . . . . . . . . . .

2.18 (a) Non-dimensional amplitude of oscillation and (b) vorticity field at four different values of the reduced velocity for a rotating parameter of $\tilde{k}_{2}=0.25$. . . . . . . . .

2.19 (a) Normalized frequency of oscillation $f^{*}$ (dashed line) and normalized frequency of vortex emission $f_{v}^{*}$ (solid line), (b) total transverse force coefficient $C_{y}$ (bold solid line), potential force coefficient $C_{p}$ (dashed line) and vortex force coefficient $C_{v}$ (solid line) and (c) FFT of the normalized vortex force coefficient $C_{v}$ at four values of the reduced velocity for a rotating parameter of $\tilde{k}_{2}=0.25 \ldots \ldots \ldots \ldots$. . . . . . . .

2.20 (a) Non-dimensional amplitude of oscillation and (b) vorticity field at four different values of the reduced velocity for a rotating parameter of $\tilde{k}_{2}=-0.25 . \ldots$. . . .

2.21 (a) Normalized frequency of oscillation $f^{*}$ (dashed line) and normalized frequency of vortex emission $f_{v}^{*}$ (solid line), (b) total transverse force coefficient $C_{y}$ (bold solid line), potential force coefficient $C_{p}$ (dashed line) and vortex force coefficient $C_{v}$ (solid line) and (c) FFT of the normalized vortex force coefficient $C_{v}$ at four values of the reduced velocity for a rotating parameter of $\tilde{k}_{2}=-0.25 \ldots \ldots \ldots \ldots$. . . . . . . .

2.22 (a) Non-dimensional amplitude of oscillation and (b) vorticity field at four different values of the reduced velocity for a rotating parameter of $\tilde{k}_{2}=-0.375 \ldots$. . . . .

2.23 (a) Normalized frequency of oscillation $f^{*}$ (dashed line) and normalized frequency of vortex emission $f_{v}^{*}$ (solid line), (b) total transverse force coefficient $C_{y}$ (bold solid line), potential force coefficient $C_{p}$ (dashed line) and vortex force coefficient $C_{v}$ (solid line) and (c) FFT of the normalized vortex force coefficient $C_{v}$ at four values of the reduced velocity for a rotating parameter of $\tilde{k}_{2}=-0.375 \ldots \ldots \ldots \ldots$

2.24 (a) Schematics of the forces acting on the cylinder in a quasi-steady situation. (b) Comparison between quasi-steady solutions and LBM numerical analysis for different values of $\tilde{k}_{1} \ldots \ldots \ldots \ldots \ldots \ldots \ldots \ldots \ldots \ldots$ 
2.25 (a) Maximum angle for rotation as a function of $U^{*}$ and $k_{1}^{*}$. (b) Maximum angle for rotation as a function of $U^{*}$ and $k_{2}^{*} \ldots \ldots \ldots \ldots \ldots \ldots$

2.26 Non-dimensional circulation of the vortices shed as a function of the reduced velocity $U^{*} .40$

3.1 Boundary separating the two types of amplitude response (three branch versus two branch) as a function of the combined mass damping parameter $\left(m^{*} \zeta\right)$ and Reynolds number. The line dividing the two regimes is defined by the non-dimensional amplitude of oscillation being $A^{*}=0.6$. It suggests approximately that below $R e \approx 500$ only the two branch response will occur independently of the value of $m^{*} \zeta$ (Govardhan and Williamson, 2006). Superimposed are shown the values of Reynolds number and $m^{*} \zeta$ for the numerical investigation led in the previous chapter $\left(R e=100\right.$ and $\left.m^{*} \zeta=0\right)$ and for the current experimental setup ( $R e=3200$ at the peak amplitude and $\left.m^{*} \zeta=0.0506\right)$. 45

3.2 Map of vortex-shedding regimes obtained from forced vibrations at $R e=4000$ as a function of the reduced velocity and the reduced amplitude of oscillation. Also, nondimensional vorticity contours of the main vortex shedding modes have been recovered from Morse and Williamson (2009). Free vibrations of circular cylinder undergoing VIV obtained in the "Antonio Barrero Ripoll" have been superimposed for $R e=3200$ at the peak amplitude and $m^{*}=11.7 \ldots \ldots \ldots \ldots \ldots$

3.3 The Griffin plot comparing peak amplitude of oscillation versus the Skop-Griffin parameter, Skop and Balasubramanian (1997) . . . . . . . . . . . . . . . . 47

3.4 Schematic elevation and plan of the free-surface water channel "Antonio Barrero Ripoll". 47

3.5 (a) Non-dimensional mean velocity contour map. (b) Turbulence intensity level contour map. These maps have been obtained at a temporary and spatially averaged velocity of $U=0.350 \mathrm{~m} / \mathrm{s} . \ldots \ldots \ldots \ldots \ldots \ldots \ldots$

3.6 Sketch of the experimental set-up (dimensions are in millimeters) of the water channel. (a) Schematical top view and side view of the water channel. (b) Photograph of a close view of the elastic system. (c) Detail of the elastic system's deformation when a translation in the transverse direction is applied. . . . . . . . . . . . . . 49

3.7 Histogram analysis for finding the most probable displacement. . . . . . . . . . . . . 53

3.8 Comparison of experimental results for the normalized amplitude variation with reduced velocity. Triangles are from Khalak and Williamson (1999) $\left(m^{*}=10.3, \zeta=0.0017\right)$, open circles are from Klamo $(2007)\left(m^{*}=6.5, \zeta=0.0015\right)$, squares are from Assi et al. (2006) $\left(m^{*}=8.1, \zeta=0.0020\right)$ and solid circles stand for present results $\left(m^{*}=11.7\right.$, $\zeta=0.0043) \ldots \ldots \ldots \ldots \ldots \ldots \ldots \ldots \ldots \ldots$

3.9 (a) Maximum non-dimensional amplitude oscillation as a function of the reduced velocity for positive values of the rotating parameter $\tilde{k}_{1}$ and (b) for negative values of

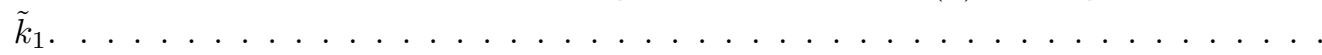

3.10 (a) Non-dimensional oscillating frequency of the cylinder as a function of the reduced velocity for positive values of the rotating parameter $\tilde{k}_{1}$ and (b) for negative values of $\tilde{k}_{1} .57$

3.11 Non-dimensional vorticity contours $\omega D / U$ for two values of reduced velocity $\left(U^{*}=5.2\right.$, $7.5)$ and four values of $\tilde{k}_{1}\left(\tilde{k}_{1}=0,1.25,-1.0,-1.875\right)$ respectively. Red color indicates clockwise circulation and blue color indicates counter-clockwise circulation. Vortical structures are highlighted with dashed line. $\mathrm{P}$ represents a pair of vortices shed per half cycle corresponding to a $2 \mathrm{P}$ mode of vortex shedding. $\mathrm{P}_{0}$ is equivalent to the previous but with the secondary vortex being qualitatively smaller (circulation intensity wise). $\mathrm{S}$ structures represents a single vortex being shed per half cycle, which corresponds to a $2 \mathrm{~S}$ mode of vortex shedding. Note that for completeness, amplitude of oscillation for each case is indicated by an arrow line. . . . . . . . . . . . . . . 
3.12 Non-dimensional vorticity $\omega D / U$ contours for $U^{*}=17$ and $\tilde{k}_{1}=-1.875$ evaluated at four different displacements of the cylinder. Red color indicates clockwise circulation and blue color indicates counter-clockwise circulation. . . . . . . . . . . . . . . .

3.13 (a) Schematics of the forces acting on the cylinder in a quasi-steady situation. (b) Average transverse force coefficient and average in-line force coefficient as a function of the rotation rate $\alpha$. Experiments to determine $C_{L}$ and $C_{D}$ have been carried out at $\mathrm{Re} \approx 9000$ which corresponds to $U^{*}=15$ for the current setup. Dashed lines represent the linear best fits. . . . . . . . . . . . . . . . . . . . . . .

3.14 Comparison between quasi-steady solution and present experimental results for $\tilde{k}_{1}=$ -2.125 .

3.15 (a) Maximum non-dimensional amplitude oscillation as a function of the reduced velocity for positive values of the rotating parameter $\tilde{k}_{2}$ and (b) for negative values of

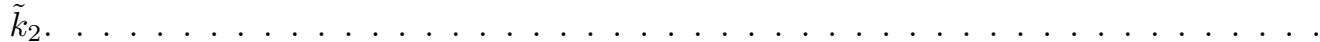

3.16 (a) Non-dimensional oscillating frequency of the cylinder as a function of the reduced velocity for positive values of the rotating parameter $\tilde{k}_{2}$ and (b) for negative values of $\tilde{k}_{2}$. 64

3.17 Non-dimensional vorticity contours for two values of reduced velocity $\left(U^{*}=5.5,7.5\right)$ and four values of $\tilde{k}_{2}\left(\tilde{k}_{2}=0,0.625,-0.625,-1.25\right)$ respectively. Red color indicates clockwise circulation and blue color indicates counter-clockwise circulation. Vortical structures are highlighted with dashed line. P represents a pair of vortices shed per half cycle corresponding to a $2 \mathrm{P}$ mode of vortex shedding. $\mathrm{P}_{0}$ is equivalent to the previous but with the secondary vortex being qualitatively smaller (circulation intensity wise). S structures represents a single vortex being shed per half cycle, which corresponds to a $2 \mathrm{~S}$ mode of vortex shedding. Note that for completeness, amplitude of oscillation for each case is indicated by an arrow line. . . . . . . . . . . . . . . . . .

3.18 Non-dimensional vorticity contours for $U^{*}=9.5$ and three values of $\tilde{k}_{2}\left(\tilde{k}_{2}=0,-0.625\right.$, $-1.25)$ respectively. Red color indicates clockwise circulation and blue color indicates counter-clockwise circulation. Vortical structures are highlighted with dashed line. P represents a pair of vortices shed per half cycle corresponding to a $2 \mathrm{P}$ mode of vortex shedding. $\mathrm{P}_{0}$ is equivalent to the previous but with the secondary vortex being qualitatively smaller (circulation intensity wise). S structures represents a single vortex being shed per half cycle, which corresponds to a $2 \mathrm{~S}$ mode of vortex shedding. Note that for completeness, amplitude of oscillation for each case is indicated by an arrow line. . . .

4.1 (a) Vorticity contour around a D-section at Re=300 with an equivalent positive angle of attack obtained through LBM method. Observe that the flow is attached in the lower part of the body inducing a lower pressure region whereas in the top part the flow if fully detached. Then, a fluid force appears in the direction of the motion thus energy is being pumped into the galloping body from the flow. (b) Steady transverse force coefficient for a square (white circles), isosceles triangle (black circles) and D-section (open squares) cross-sections. Lines correspond to the fitting curves. . . . . . . . . . .

4.2 Steady transverse force coefficients $C_{y}$ for different rectangular cross-sections with the corresponding four main characteristic amplitude responses. . . . . . . . . . . . .

4.3 Schematics of the fluid forces appearing on the galloping body and of the induced angle of attack obtained through the quasi-steady hypothesis. . . . . . . . . . . . . .

4.4 (a) Sketch of the spring-mounted galloping body in cross-flow and linked to an electromagnetic generator. (b) Equivalent electrical circuit of the electromagnetic generator.

4.5 Universal plot of the efficiency versus the flow velocity. . . . . . . . . . . . .

4.6 Variation with the reduced velocity $U^{*}$ of the optimal electrical load resistance (a), output electrical power (b), efficiency $(\mathrm{c})$, and $\beta(\mathrm{d}) \ldots \ldots \ldots \ldots$ 
4.7 (a) Efficiency variation with reduced velocity for optimal electrical load resistance (solid line) and for two different fixed values of the electrical load resistance (dashed lines). White circles denote the local maximum efficiency for a given $R_{L}$ whereas black circle denotes the absolute maximum efficiency. Note that the efficiency variation with reduced velocity for optimal electrical resistance (solid line) is the envelope of all local maximum efficiency points. (b) Output power variation with reduced velocity for optimal electrical load resistance (solid line) and for two different fixed values of the electrical load resistance (dotted line) . . . . . . . . . . . . . . . . .

4.8 (a) Optimal electrical load resistance variation with $\beta$ computed numerically (solid line) and given by analytic approximation (Eq. 4.42). It is also shown (dashed line) the optimal value predicted when inductance effects are not considered (Eq. 4.27). (b) Relative error variation with $\beta$ for the analytic approximation when inductance effects are not considered (dashed line) and for the analytic approximation when inductance effects are considered (dotted line). $U^{*}=14.94$ and parameters listed in Table 4.2. . .

4.9 Schematic of a galloping prism prone to galloping transverse to the incident airflow.

4.10 (a) Side view of the galloping prism attached to a cantilever beam with piezoelectric patches. (b) Detail of the thickness of the triple-layer piezoelectric mounted on the

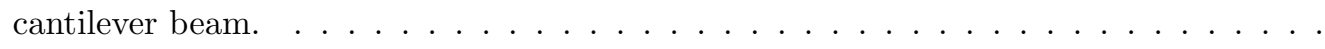

4.11 Power harnessed for three different incident velocities of the airflow. Open squares correspond to experiments from Zhao et al. (2013), solid lines represent power harnessed taking into account corrections due to the deflection of the cantilever beam's tip. . . .

4.12 (a) Power harnessed for constant electric load $\left(R_{l}=105 \mathrm{k} \Omega\right)$ over a range of incident velocities of the airflow.(b) Energy efficiency conversion for constant electric load. Open squares correspond to experiments from Zhao et al. (2013), solid lines represent power harnessed taking into account corrections due to the deflection of the cantilever beam's tip. (c) Relation between the efficiency of power harnessed taking into account the effect of the deflection at the tip of the cantilever beam with respect to that of pure transverse galloping. . . . . . . . . . . . . . . . .

4.13 (a) Contour map of the efficiency of power harnessed as a function of the incident velocity $U(\mathrm{~m} / \mathrm{s})$ and of the electric load $(\Omega)$. (b) Power harnessed given in miliWatts as a function of the incident velocity and the electric load selected. In dashed lines Eq. 4.60 is plotted which yields the maximum efficiency $\eta_{E}$ for each value of $U^{*}$. Highlighted as a black dot is given the maximum global efficiency $\eta_{E}^{G, \max }$ which is obtained at a given value of $\beta_{\max }^{G}$ and $U_{\max }^{* G}$ as given by Eqs. 4.62 and 4.63 respectively. . . . . . . . .

4.14 Defining sketch of the three possible dual-mass configurations which are differentiated by the situation of the harvesting damper. . . . . . . . . . . . . . .

4.15 Universal efficiency curve valid for all mechanical configurations. $\tilde{\eta}=\eta^{D M} / \eta_{\max }^{D M}$ and $\tilde{U}=U^{*} / U_{g}^{* D M} \ldots \ldots \ldots \ldots \ldots \ldots \ldots \ldots \ldots$

$4.16 \psi_{D M}$ dependence with mechanical properties of the dual-mass systems $(\Omega, \mu)$ for the first (a) and second (b) mode. The first mode always involves values of $\omega^{*}>1$ whereas the second one involves values of $\omega^{*}<1 \ldots \ldots \ldots \ldots \ldots$

$4.17 \chi_{D M}$ dependence with mechanical properties of the dual-mass systems $(\Omega, \mu)$ for the first mode $\omega^{*}>1$ (a) and for the second mode $\omega^{*}<1(\mathrm{~b}) \ldots \ldots \ldots$. . . . . . .

4.18 Left: Efficiency variation with the reduced velocity for the single mass system with the dual-mass system and dual mass-system (HBM and numerical solution). Right: power extracted for the single mass and dual-mass configurations. . . . . . . . . . . . 
4.19 Sketch showing a potential practical realization, which includes a galloping prism (A), springs (B), a linear guide (C) transverse to the flow direction, the secondary mass (D), the stator (coil) part of the electromagnetic generator, and a permanent magnet array (F). A 3D view is shown in (a) whereas a front view is shown in (b). . . . . . . . . . 109

4.20 (a) Variation of $\psi_{D M}$ with $\Omega$ and $\mu$ for the $\mathrm{C} 2$ configuration ( $\omega^{*}>1$ mode). (b) Variation of $\psi_{D M}$ with $\Omega$ and $\mu$ for the C2 configuration $\left(\omega^{*}<1\right.$ mode). . . . . . . 112

4.21 Variation of $\chi_{D M}$ with $\Omega$ and $\mu$ for the $\mathrm{C} 2$ configuration ( $\omega^{*}>1$ mode) . (b) Variation

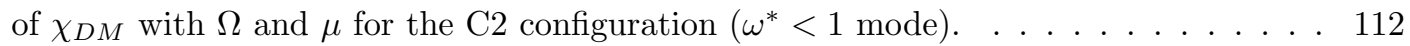

4.22 Schematics of the forces acting on the galloping prism in a quasi-steady situation. . . . 114

$4.23 C_{D}$ and $C_{L}$ static static coefficients dependent on the angle of attack computed through numerical LBM computations for a D-section at $R e=100$. For comparison purposes, results from Bhinder et al. (2012) are presented. . . . . . . . . . . . . . . . 115

4.24 Transverse force coefficient $\tilde{C}_{y}$ as a function of $\alpha_{F}$ for different values of $K_{1} \ldots \ldots$

4.25 Variation of $\tilde{a}_{1}$ and $\tilde{a}_{3}$ as a function of $K_{1}$. . . . . . . . . . . . . . . . . . . . . . . 117

4.26 Comparison of the amplitude of oscillation $A^{*}$ as a function of the reduced velocity for different values of $K_{1}$ and $\zeta \ldots \ldots \ldots \ldots \ldots \ldots \ldots \ldots$

4.27 Slope of $A^{*}$ for high enough values of $U^{*}$ as a function of $K_{1}$ calculated from the quasisteady model. Superimposed are given the slope obtained through LBM computations. 119

4.28 Maximum efficiency of energy harvesting as a function of $K_{1}$. . . . . . . . . . . . . . . 120

4.29 Slope of $A^{*}$ for high enough values of $U^{*}$ as a function of $K_{1}$ for different values of $n$. 121

4.30 Maximum efficiency of energy harvesting as a function of $K_{1}$ for different values of $n$. 121

4.31 Vorticity contour for a D-section at $R e=100$ without actuation for a reduced velocity of $U^{*}=4$, a reduced mass $m^{*}=10$ and a non-dimensional damping parameter of

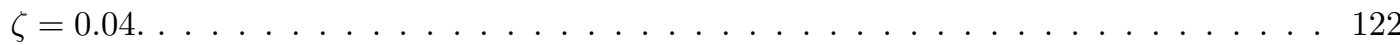

4.32 Vorticity contour for a D-section at $R e=100$ with actuation $\left(K_{1}=1, n=1\right)$ for a reduced velocity of $U^{*}=4$, a reduced mass $m^{*}=10$ and a non-dimensional damping parameter of $\zeta=0.04 \ldots \ldots \ldots \ldots \ldots \ldots \ldots$

4.33 Variation of $\tilde{a}_{1}, \tilde{a}_{3}, \tilde{b}_{1}$ and $\tilde{b}_{3}$ as a function of $K_{2} \ldots \ldots \ldots \ldots \ldots \ldots$

4.34 Maximum efficiency of energy harvesting as a function of $K_{1}$ for different values of $n$. 124

A.1 Reduced velocity at which the maximum efficiency is obtained as a function of $\delta$ times the initial reduced galloping velocity. Dashed line represents the asymptotic solution for $\delta U_{g}^{*} \gg 1 \ldots \ldots \ldots \ldots \ldots \ldots \ldots \ldots \ldots \ldots$ 


\section{List of Tables}

2.1 Comparison of Strouhal number, in-line average force coefficient and maximum transverse force coefficient for a static cylinder at $R e=100 \ldots \ldots$. . . . . . . . . . 21

4.1 Static fluid dynamic characteristics of different croos-sections. . . . . . . . . . . . . 75

4.2 Physical properties of galloping body and the electromagnet generator. Values of the coefficients $a_{1}$ and $a_{3}$ are taken from Barrero-Gil et al. (2010). . . . . . . . . . . . . 84

4.3 Physical properties considered in the example for the galloping body and the piezoelectric beam as obtained from Zhao et al. (2013). Values of the coefficients $a_{1}$ and $a_{3}$ are taken from Table 4.1 for the square section. . . . . . . . . . . . . . . . . .

4.4 Physical parameters for the single mass and dual-mass systems considered in the presented practical example. . . . . . . . . . . . . . . . . . 108 


\section{Chapter 1}

\section{Introduction}

\section{$1.1 \quad$ Introduction}

Flow induced vibrations (FIV) of bluff bodies (bodies in which as a result of its shape, have a separated flow over a substantial part of its surface) are common in nature as well as in man-made constructions. To name a few examples of the former, the wind-induced fluttering of leaves and tree branches as well as waving motions of wheat fields (De Langre, 2008), or in the locomotion of animals (birds or fishes) could be mentioned. Man-made examples include a wide range of cases belonging to different fields of application: musical instruments; aerospace engineering: flutter of aircraft surfaces, blades of turbo-machinery, wind turbine blades; civil engineering: light long-span bridges, high-rise buildings, traffic signs, slender chimneys or skyscrapers, lines of power transmission, tubes of steam generators and heat exchangers used at nuclear power-generation; naval engineering: mooring lines , oil drilling, flexible structures in petroleum production, etc.

From a historical perspective, interest in FIV stretches back perhaps 3000 years, where the aeolian harp might be one of the first examples of exploitation of these vibrations. The first documented realizations of the existence of the phenomena of vortex shedding was first documented back in the $14^{\text {th }}$ century in a painting attributed to Giovanni da Modena in Bologna or in the $15^{\text {th }}$ century, when Leonardo da Vinci sketched vortices in the wake of a pile in a stream, but it was with the arrival of new lighter materials that the field of FIV experienced its main development. One might recall the episode of the Tacoma Narrows bridge. Since the time the deck was built, it began to oscillate vertically for incident wind velocities well under the design one and finally, the bridge's main span collapsed in the morning of November 7, 1940, only four months after the bridge was inaugurated and opened to traffic. The bridge's collapse had a long-term effect on both science and engineering. Early investigations presented the event as a forced resonance, with vortices being shed at a frequency that matched the bridge's natural structural frequency. Since then it was proven that the ultimate cause of failure was a self-excited phenomenon, the so called torsional galloping (Parkinson, 1989). Its failure became an incentive on the research of aerodynamics and aeroelasticity of bluff bodies, not only of long-span bridges but also for other slender structures in which FIV may lead to the appearance of self-excited oscillations which could potentially arise complications.

From the point of view of basic research, FIV of bluff bodies is a very interesting field with very rich phenology appearing due to the complex interaction between the flow around the body (usually detached flow with large-scale vortex formation) and the elastic response of the body. Non-linear resonances, hysteresis phenomena, lock-in, quasi-periodicity or other phenomena has been traditionally observed. The flow around the body (either stationary or in motion) induces fluid forces on the body's 
surface. These fluid forces might modify the shape or dynamics of the body (orientation, velocity) which successively changes the flow around the body, thus a strong coupling between the flow and the body in question appears, requiring an integral description of the problem. The number of parameters involved in the problem are usually not small since the problem is governed by parameters describing the flow as well as parameters describing the body's dynamics. Usually many parameters have to be taken into account in order to fully describe the phenomena. Among others one could mention here the mass ratio (mean density of the body over fluid density), mechanical dissipation of the body, the reduced velocity, the Reynolds number, roughness of the body, flow confinement, aspect ratio, etc. The number of contributions to the field, both analytical-numerical and experimental, is enormous and has increased exponentially with time. An all-inclusive introduction to the field is almost not possible, and to some extent without sense for the scope of this thesis. In this thesis only some specific aspects of the FIV field will be investigated, so that a review of the specific subject will be presented at the time. For an introduction to the field of FIV the reader is referred to the books of Blevins (1990), Naudascher and Rockwell (1994), or Paidoussis, De Langre, and Price (2012).

From the practical side, FIV are a main concern in many engineering structures, since they can reduce their lifetime by fatigue loading, compromise their service, or put at risk their integrity. Just as an example, we can cite here that the cumulative damages incurred over a decade associated to the fluid-elastic instabilities of cylinder arrays in cross-flow was estimated at 1000 million of dollars (Païdoussis, 2006). Recently, the field of interest has been notoriously enlarged since FIV have been considered as a mean to extract useful energy. If the elastic body is correctly designed, it can extract efficiently energy from the surrounding flow. Through a FIV phenomenon part of the incoming kinetic energy in the flow is transformed into mechanical energy of the body's oscillations, then the mechanical energy of the body can be converted into electrical energy if some kind of transducer is present to extract mechanical energy from the oscillations. In essence, it is possible to extract useful energy from the fluid flows by transforming kinetic energy of the incident flow into mechanical energy as oscillations of the structure which in turn can be converted into electrical energy by means of electromagnetic, electrostatic or piezoelectric transducers.

\subsection{A new paradigm, flow induced vibrations as an energy harvesting mechanism}

In the last few years there has been an increase on the awareness of the environmental impact of fossil fuels, leading to a major effort in developing and diversifying the energy production through renewable energy technologies.

Geophysical flows have been found to be an immense natural reservoir of energy present throughout the world, being present as wind, river, oceanic or tidal flows among others. Most of the concepts operating nowadays that take advantage from fluid flows to harness energy rely on horizontal-axis turbines. The lift forces that appear on the turbine blades produce a torque which energizes an electromagnetic generator. One of the characteristics of geophysical flows (independently of the method used to extract energy) is that the energy density in these flows is relatively low (being the kinetic energy of the order of $1-10 \mathrm{~kW} / \mathrm{m}^{2}$ ) making it difficult to replace conventional power-plants (coal, nuclear, combined cycle), unless large systems are employed. However, it is well suited for a distributed energy distribution concept, where small to medium power plants are built next to the destination of the consumption of the required power. For these reduced scales, turbine technologies are inefficient (due to the operating flow velocities), thus encouraging to seek for new technologies to harvest energy from fluid flows with big differences in their characteristics (namely, density of the fluid, mean velocities, turbulence, etc.). 
With this motivation in mind, FIV have been investigated as promising candidates in order to extract fluid kinetic energy of geophysical flows through electromagnetic or piezoelectric harvesting systems. Several concepts introduced in the last decade work in this direction and should be referenced here. From a fundamental point of view, where the flow-induced vibration phenomenon is analyzed without any specific transduction system, we must mention the work of Bernitsas et al. (2008) who described a way to use the oscillations induced by vortex shedding from a spring-mounted circular cylinder under the action of a water current with their VIVACE design. The influence of some key parameters like the mass ratio, the mechanical damping, the Reynolds number (Raghavan et al., 2007), and the cylinder's aspect ratio (length to diameter ratio) in the electrical power was experimentally investigated in a re-circulating water channel. VIV potential for energy extraction has also been studied by Grouthier et al. (2013), Mackowski and Williamson (2013), Barrero-Gil et al. (2012), or Sanchez-Sanz et al. (2009). In past years, several research has been carried out considering a piezoelectric material as a transduction means to produce small quantities of electrical power (of the order of mili-watts) from VIV of a circular cylinder under the action of an airstream. For example, Abdelkefi et al. (2012a) presented a theoretical analysis using a wake-oscillator model to describe fluid forces on the oscillating circular cylinder which is appropriately coupled with the cylinder and piezoelectric dynamics. Additionally, Akaydin et al. (2012) also presented an experimental approach. Energy harvesting from oscillations of a circular cylinder in the wake of a fixed upstream cylinder has been experimentally studied by Nishi et al. (2014) and by Jung and Lee (2011), where electromagnetic conversion was used. Abdelkefi et al. (2013a) carried out experiments to investigate the effects of wake galloping on the range of flow speeds over which a galloping-based piezo-aeroelastic energy harvester can be effectively used. Two different upstream cylinders and a wide range of spacing between the upstream and downstream cylinders were considered. The use of flutter phenomena for energy extraction has also been studied by Tang et al. (2009) or Zhu et al. (2009). Modeling and experimental results are given by Bryant and Garcia (2011), where piezoelectric conversion was employed. Zhu and Peng (2009) in their STINGRAY concept took advantage of the coupled-mode flutter of a rigid heaving and pitching airfoil (similar concept from Kinsey and Dumas (2008) should be acknowledged). The energy-harvesting eel proposed by Allen and Smits (2001) should be mentioned, where a piezoelectric membrane is placed in the wake of a bluff body (Taylor et al. (2001)). When the mass and elastic properties of the membrane are appropriately chosen, the large-scale vortex street formed behind the body induces significant oscillations in the membrane that can be converted into electricity. Finally, the idea of using transverse galloping as a way of extracting energy in a renewable way from a flow was first proposed by Barrero-Gil et al. (2010), proving higher energy transfer and for a larger set of reduced velocities compared to VIV. As a result, the GALOPE project appeared with the objective to design energy harvesters taking advantage of transverse galloping (Barrero-Gil et al. UPM P120130288 and patented under EP 2932091, US 2015330358) in order to investigate the main parameters and to optimize the energy harvesting from transverse galloping phenomenon.

Note that traditionally FIV were undesired and generally considered to be dangerous, since oscillations appearing on structures not meant to might provoke from short-term destruction of the structure up to long-term wear problems like fatigue. Therefore, most of the research lead on fluidsolid instabilities in the past dealt with the pernicious effects of these oscillations on the structures and were motivated by the identification of the phenomenon in order to avoid or control its presence. Thus, most of the existing literature in the past on such fluid-solid interactions emphasize on linear analysis as one was interested on determining when would such oscillations appear and on minimizing the amplitude of oscillations (and the energy transfer from the flow to the body). Also, much of the previous research dealt with weakly damped systems as it is a usual characteristic of civil engineering structures. On the other hand, energy harvesting from FIV phenomena looks for maximizing the energy transfer from the flow to the body and with situations of high damping with high amplitude level 
of oscillations. This requires a non-linear analysis of importantly damped bodies where amplitude of oscillation ought to be enhanced and promoted. While the fundamental notions responsible for the vibrations are well known, non-linear dynamics and their susceptibility to parameters involved, including the energy extraction process, remains in many cases to be better investigated. During the design process of an energy harvester, one ought to seek for maximum power production, which correspondingly produces a loss in the mechanical energy of the fluid-solid system, and consequently modifies its dynamics. Two main goals are therefore sought in this research topic, (i) increase the fundamental knowledge on large amplitude FIV phenomena and (ii) to properly design the energy harvesting device in order to maximize its efficiency as well as to better design optimization strategies.

\subsubsection{Considerations on energy harvesting via FIV}

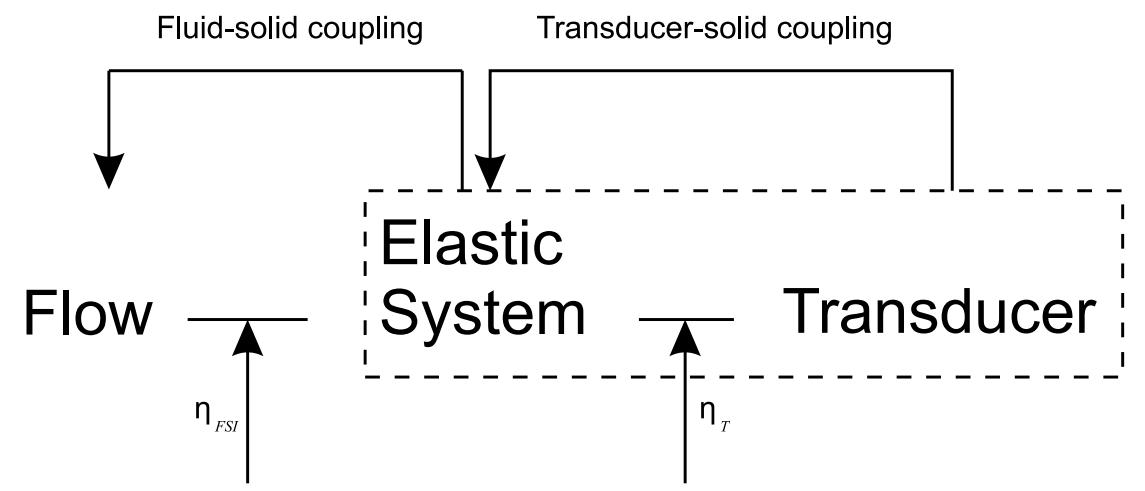

Figure 1.1: Simplified schematics of the energy harvesting process from FIV.

As previously mentioned, energy harvesting from FIV phenomena is a fully coupled non-linear problem with many parameters being involved. Fig. 1.1 shows schematically the process of energy extraction. As can be seen, a typical energy harvester system can be depicted for its three main elements, (i) the flow from where it is sought to extract its kinetic energy, (ii) the mechanical system, namely the bluff body immersed in the flow, prone to suffering fluid forces and whose design will mark very importantly the maximum efficiency of energy harvesting available, with the stiffness required to provide with restoring forces (springs, cantilevered beams among others). Finally, (iii) the transducer, where the mechanical energy of the system is transformed into electrical power (through electromagnetic, electrostatic or piezoelectric means). These main elements that configure a typical harvester system are heavily coupled with backlash forces appearing between them. Mainly, two basic interactions appear: a fluid-solid and a solid-transducer interaction with an overall net transfer of energy among them.

To center the discussion, an efficiency of energy harvesting should be defined, which gathers information about the process of energy transfer from the fluid flow and the final transducer. The amount of energy that can be captured through a FIV system is related to the size of the system as well as to the incoming cross-flow (most importantly density $\rho$ and steady velocity $U$ ). Therefore, it is convenient to rely on non-dimensional analysis to establish optimal configurations regarding the power harvested. In particular, the efficiency of energy extraction can be defined as the fraction of energy harvested $\left(P_{E}\right)$ compared to the kinetic energy of the flow per unit time ${ }^{1}\left(P_{K}\right)$ as

$$
\eta_{E}=\frac{P_{E}}{P_{K}}=\frac{P_{E}}{1 / 2 \rho U^{3} A_{s}},
$$

\footnotetext{
${ }^{1}$ Other formulations for the efficiency have been described in the available literature, including scaling the harvested energy to the peak kinetic energy of the solid (Dunnmon et al., 2011), or the mean energy of the body in a period (Doaré and Michelin, 2011).
} 
where $A_{s}$ is a reference area to be taken into consideration. Defining appropriately $P_{K}$ is fundamental on the results obtained as well as on biasing optimal configurations yielded. In particular, scaling the energy production with the area swept by the body during its oscillation has a fundamental advantage as its definition is similar to horizontal-wind/water turbines, where the harnessed power is compared to the energy flux through the turbine disk. With this definition, it is also possible to explore an equivalent to the Betz limit for horizontal-wind/water turbines. Nonetheless, this formulation for FIV has a main drawback, as it requires to determine the area swept unambiguously which is not always directly achievable as it is usually the solution of the problem. Another usual formulation, as in Barrero-Gil et al. (2010) or Grouthier et al. (2013), compares the energy extracted to the kinetic energy flux to an area equivalent to the frontal surface of the body immersed in the flow and suffering FIV. Note that both efficiencies might yield different results of optimal configuration as the latter stresses the importance of the maximum power extracted while the former stresses the importance of maximizing the energy harvested per unit length in the transverse direction.

Furthermore, $\eta_{E}$, either defined with the swept area or with frontal characteristic area of the body, can be splitted as

$$
\eta_{E}=\frac{P_{M}}{P_{K}} \frac{P_{E}}{P_{M}}=\eta_{F S I} \eta_{T},
$$

where $\eta_{F S I}=P_{M} / P_{K}, \eta_{T}=P_{E} / P_{M}$ and $P_{M}$ is the energy stored as mechanical energy of the body's oscillations. Regarding $\eta_{F S I}$, it basically collects the efficiency of energy transfer from the fluid's kinetic energy into the solid's mechanical energy and therefore evaluates the capacity of the body to capture energy from the cross-flow. Necessarily, $\eta_{F S I}$ is most influenced by the type of FIV phenomena under consideration. For example, Vortex-Induced Vibrations, flutter or transverse galloping will all yield different levels of efficiency of energy conversion. Important parameters that need to be mentioned that affect heavily $\eta_{F S I}$ are the cross-section geometry, fluid speed, roughness of the body, structural mass, damping and stiffness or confinement of the flow to name but a few. It is possible to gather much information by analyzing $\eta_{F S I}$, e.g. if a given cross-section is ill-suited for a given FIV and thus yields low values of $\eta_{F S I}$, needless to say, the overall the energy extraction efficiency will consequently also be low.

With respect to $\eta_{T}$, it basically accounts for the final electrical energy harnessed from mechanical energy stored in the body namely as oscillations. The type of transducer used to harvest energy will doubtlessly account as the most important factor. As will be shown, different transducers affect the dynamics of the body differently (namely by modifying the overall stiffness or damping of the body) which correspondingly affects the fluid-solid coupling. It is decisive to match properly the transducer properties for each case and to correctly choose the type of transducer used (basically based on electromagnetic, piezoelectric or electrostatic means).

As seen in Fig. 1.1, the solid-transducer coupling may be substituted by an equivalent mechanical configuration, since it always has an overall mechanical effect from the flow's perspective. As a first step, energy harvesting accounts for a loss of net energy from the solid, hence, the coarsest way of modeling such interaction is by introducing a pure viscous damping (Peng and Zhu (2009), Tang et al. (2009) or Barrero-Gil et al., 2010). This way of representing the solid-transducer coupling is a simple way of determining the amount of energy that can be harvested for a particular fluid-solid interaction system taking into account the main physical process underlying, namely the loss of mechanical energy of the body (especially, as body's oscillations). In the limit of non-existing damping term, the energy harvested is insignificant, thus to obtain higher efficiency, one should increase the equivalent damping of the transducer, which on the other hand will affect the dynamics of the body. Basically, as the damping of the transducer is increased, the amplitude of vibration will be reduced and the body's mechanical energy will decay. If the damping is too large, the system's efficiency is penalized, the fraction of mechanical energy of the solid transformed to electrical energy is still high, however, the available mechanical energy of the solid becomes limited. Thus, an optimal configuration has to be 
sought to maximize the efficiency of energy harvesting and to do so, a better understanding of the fully coupled fluid-solid-transducer interaction has to be developed.

Let us introduce a simplified model to better illustrate the importance of correctly matching the properties of a generic transducer. Namely, if the backlash forces of the transducer on the solid can be considered to be proportional to an equivalent viscous damping and to a stiffness term as $F_{T}=c_{e q} \dot{y}+k_{e q} y$, where $y$ is a transverse displacement and $\dot{y}$ its transverse velocity. Then, the mean power extracted can be described as

$$
P_{E}=\frac{1}{T} \int_{0}^{T} F_{T} \dot{y} d t=\frac{1}{T} \int_{0}^{T} c_{e q} \dot{y}^{2} d t+\frac{1}{T} \int_{0}^{T} k_{e q} y \dot{y} d t,
$$

where $T$ accounts for a period of oscillation. If sinusoidal oscillations can be considered for the steady state solution a further level of simplification can be achieved for the mean power extracted

$$
P_{E}=\frac{1}{T} \int_{0}^{T} c_{e q} \dot{y}^{2} d t=\frac{c_{e q} A^{2} \omega^{2}}{2},
$$

where $A$ is the amplitude of oscillation of the body and $\omega$ the frequency of oscillation. Thus, in order to optimize the power output, one seeks to increase the amplitude of oscillations as well as to increase the transducer's gain $c_{e q}{ }^{2}$. Nonetheless, the latter cannot be increased without affecting the dynamics of the oscillator, namely by reducing the amplitude of oscillation. Differentiating Eq. 1.4 with respect to $A$ and $c_{e q}$ it is possible to determine how variation of different parameters affect the overall energy extraction from the current,

$$
\begin{aligned}
& \frac{d P_{E}}{d A}=\frac{\partial P_{E}}{\partial A}+\frac{\partial P_{E}}{\partial c_{e q}} \frac{\partial c_{e q}}{\partial A} \\
& \frac{d P_{E}}{d c_{e q}}=\frac{\partial P_{E}}{\partial c_{e q}}+\frac{\partial P_{E}}{\partial A} \frac{\partial A}{\partial c_{e q}}
\end{aligned}
$$

From Eq. 1.5a, generally speaking one can assess that $\partial c_{e q} / \partial A=0$ and that $\partial P_{E} / \partial A>0$, thus for an increment of the amplitude of oscillation, there is an increase in the power harnessed. As will be seen in the study of Vortex-Induced Vibrations of an alternating rotating circular cylinder (Chapters 2 and 3), one of the objectives to look for efficient energy harvesting strategies is to enhance oscillations' amplitude as they are intrinsically related to an increase in the power extracted. From Eq. 1.5b, it can commonly be assured that $\partial P_{E} / \partial c_{e q}>0$, also that $\partial P_{E} / \partial A>0$ and that $\partial A / \partial c_{e q}<0$. Therefore, it is complicated to determine unequivocally the optimal value of $c_{e q}$ to harness the maximum power. To do so, an integral evaluation of the fully couple fluid-solid-transducer has to be performed which involves modeling appropriately all of the elements involved.

\subsection{VIV and transverse galloping}

The number of different phenomena that comprise FIV is vast and several ones have been explored as a mean to extract energy from geophysical flows. This thesis will be focused on the optimization of the energy harvesting process from Vortex-Induced Vibrations (VIV) and transverse galloping phenomena. Notoriously, these two FIV problems constitute the basis for the analysis of many related problems that usually appear when an elastic bluff body (not streamlined) is immersed in a cross-flow. In real situations, the elastic body under consideration is three dimensional and the incoming flow is nonstationary and three dimensional. Having said that, both VIV and transverse galloping are normally associated to slender elastic bluff-bodies with a preferential displacement direction (transverse to the

\footnotetext{
${ }^{2}$ Though it could also be sought to increase the frequency of oscillation $(\omega)$ so as to maximize the power extraction, normally variations in $\omega$ are not as significant and could even be harmful, specifically in resonant types of FIV.
} 
incoming flow). That is, for the study of VIV or transverse galloping, via experiments or numerical simulations, it is common to consider the simplified case of a rigid body (mostly and most importantly a circular cylinder in VIV) whose degrees of freedom have been reduced from six to often one. In other words, most of the research that has been done in the past regularly considers the canonical problem of a rigid prism elastically-mounted free to oscillate transversely ( $y$ direction) to the incident current (see Fig. 1.2). To focus the discussion, a brief description of the VIV and transverse galloping phenomena is here presented. Phenomenology of VIV and transverse galloping are very rich and varied, thus it is not the goal of this brief summary to be exhaustive but to introduce some main concepts that will be useful for the rest of the thesis. For further details, the reader is referred to the authoritative reviews by Williamson and Govardhan (2004), Sarpkaya (2004) for VIV, and Parkinson (1989) for transverse galloping.

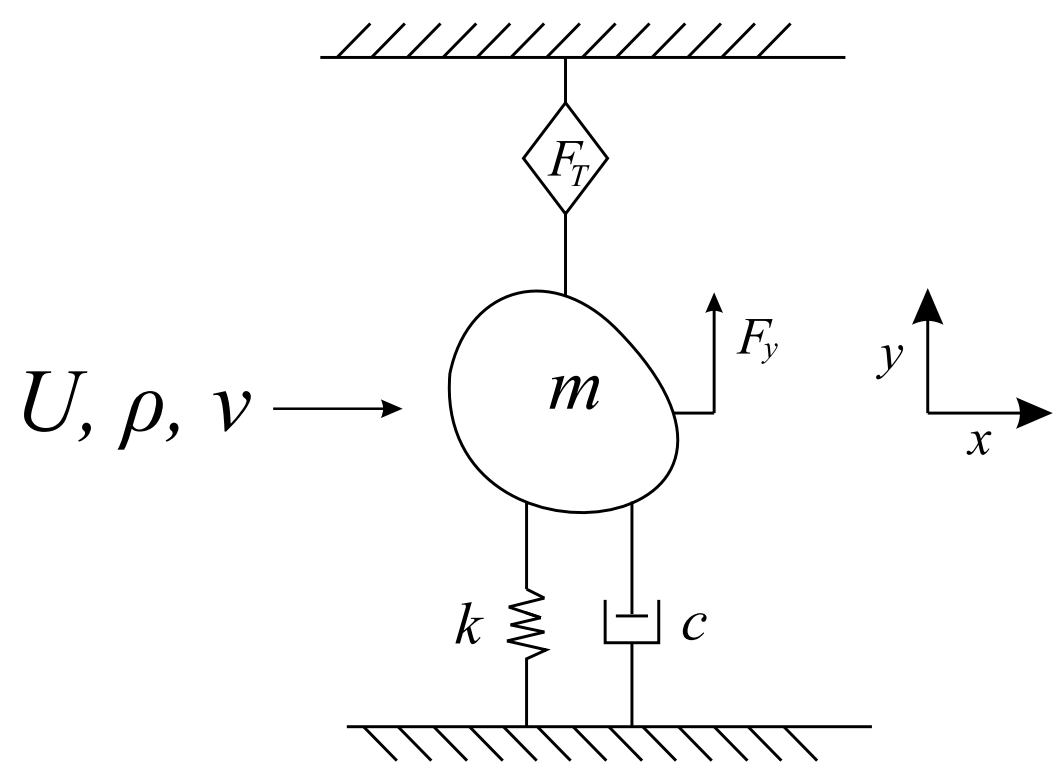

Figure 1.2: Elastically-mounted body free to oscillate tranversely to the cross-flow.

\subsubsection{Parameters governing VIV and transverse galloping}

Taking into consideration the fluid-elastic problem schematically shown in Fig. 1.2, where oscillations takes place along the transverse to the flow direction $(y)$ a brief review of the main parameters that appear in the problem may be given.

1. The incoming flow is characterized by its density $\rho$, its kinematic viscosity $\nu$, its main incident velocity $U$. The unperturbed flow is considered steady and turbulence will not be taken into account as a first approximation.

2. The main parameters that take part regarding the solid are: its geometry represented by two characteristic lengths, the characteristic length of the cross-section geometry $D$ and a perpendicular length $L$ with normally $L / D \gg 1$ for slender structures. Also, the mass per unit length of the structure $m$, its structural damping per unit length $c$ and stiffness per unit length $k$. Thus a natural frequency of oscillation can be described as $\omega_{N}=(k / m)^{1 / 2}$.

3. Parameters related to the transducer vary importantly depending on the type of transducer used. Nevertheless, as previously introduced, it can be modeled through an equivalent damping term $c_{e q}$ and stiffness term $k_{e q}$ acting on the solid as $F_{T}=c_{e q} \dot{y}+k_{e q} y$. 
Therefore, taking $m, D$, and $\omega_{N}$ as characteristics scales, the non-dimensional amplitude of oscillation dependence with these parameters can be expressed as

$$
A^{*}=\frac{A}{D}=G\left(U D / \nu, U / \omega_{N} D, L / D, m / \rho D^{2}, c / 2 m \omega_{N}, c_{e q} / 2 m \omega_{N}, k_{e q} m / \omega_{N}^{2}\right) .
$$

And the transverse fluid force coefficient dependence

$$
C_{y}=\frac{F_{y}}{1 / 2 \rho U^{2} D L}=H\left(U D / \nu, U / \omega_{N} D, L / D, m / \rho D^{2}, c / 2 m \omega_{N}, c_{e q} / 2 m \omega_{N}, k_{e q} m / \omega_{N}^{2}\right) .
$$

From the previous relation, some very important non-dimensional parameters that will be useful for the rest of the thesis can be described:

- Reynolds number, $R e=U D / \nu$.

- Reduced velocity, $U^{*}=U / \omega_{N} D$, which compares the characteristic timescale of oscillation $\omega_{N}^{-1}$ with the characteristic timescale of residency of the fluid $D / U$.

- Reduced mass, $m^{*}=m / \rho D^{2}$, which compares the mass per unit length of the structure with the displaced mass of the fluid under consideration. Thus, it is related to the ratio between the densities of the structure and of the fluid.

- Non-dimensional mechanical damping term $\zeta=c / 2 m \omega_{N}$.

As well as the non-dimensional transducer-damping term and non-dimensional transducer-stiffness term.

\subsubsection{VIV}

Vortex-Induced Vibrations (VIV) of a circular cylinder are the canonical example of Fluid-Solid Interactions in which there is a resonance coupling between the elastic system and the wake around the bluff body. VIV are not uncommon and they are responsible for undesired vibrations such as in heat-exchanger tubes in nuclear reactors, in risers of offshore oil rigs, skyscrapers, bridges or in power lines. For high enough Reynolds number (say, larger than 50), the flow separates from the cylinder surface generating an unsteady broad wake, where large-scale vortices are shed periodically from the body surface (Von-Karman vortex street) which leads to an alternating fluid force on the body. The shedding frequency of the vortices is related to the undisturbed flow speed and the size and shape of the body

$$
\mathrm{St}=\frac{f_{S t} D}{U},
$$

where St is the Strouhal number (Blevins (1990)), $U$ the undisturbed flow velocity, $D$ the body's characteristic length and $f_{S t}$ is the frequency of vortex emission.

As the flow velocity is increased from zero, the vortex shedding frequency increases almost linearly and there is a flow velocity at which vortex shedding has a frequency close to the cylinder's natural frequency of oscillations and, for low values of the mass and mechanical properties, significant oscillations can be induced in the body. When the cylinder is oscillating a complex interaction between the oscillating body and flow field around it develops, where two features must be outlined: (i) there is a range of flow velocities where vortex shedding frequency is synchronized with the frequency of oscillation (lock-in regime), giving for significant oscillations and (ii) the cylinder response may exhibit abrupt changes, with jumps in oscillation amplitude and in the fluid forces acting on the body. This abrupt changes are associated to changes in the vortex shedding pattern, (iii) the maximum amplitude of oscillations is self-limited (typically amplitude of oscillations does not exceed a diameter), in spite of the mechanical properties (mass ratio and damping ratio) becoming very small. To get an initial 
idea about the rich VIV phenomenology, probably the best is to refer to experiments. Experimental results from Nemes et al. (2012) are presented in Fig. 1.3 which constitute a typical response (non-dimensional amplitude and frequency of oscillation) for the steady-state oscillations caused by VIV phenomenon of an elastically-mounted rigid circular cylinder in their free-surface water channel (a video of VIV of a circular cylinder at the "Antonio Barrero Ripoll" water channel is available at www.tfslab.es/david). Some remarks can be made with regard to the VIV response observed:

(a)
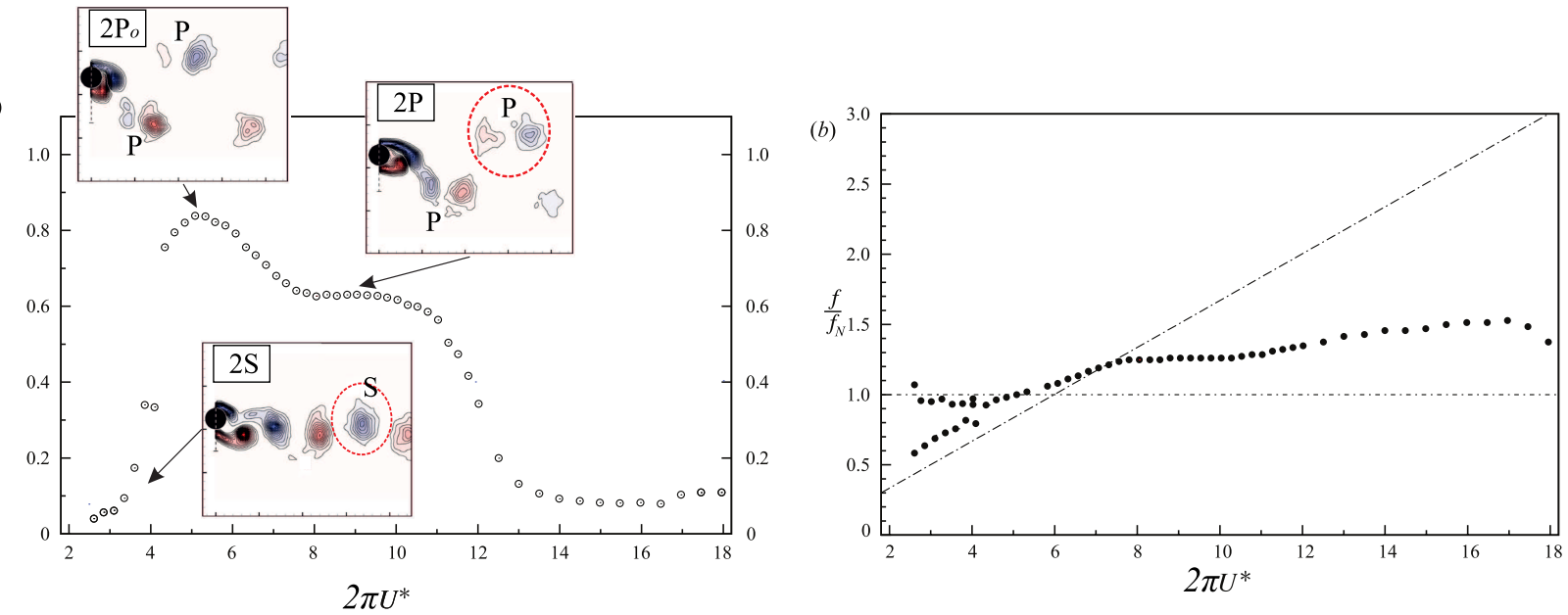

Figure 1.3: (a) Amplitude response and (b) frequency response from VIV oscillations at $m^{*}=2.2$ from Nemes et al. (2012). Superimposed non-dimensional vorticity contours for different wake patterns from Morse and Williamson (2009).

- There is no significant oscillations until a value of the reduced velocity close to $2 \pi U^{*}=4$. The normalized frequency of oscillations $\omega^{*}=\omega / \omega_{N}$ indicates that these initial oscillations have two different leading frequencies, close to the frequency given by the Strouhal law and the natural frequency.

- When $2 \pi U^{*} \approx 4.5$ an abrupt change in amplitude of oscillations is observed. This change is due to a change in the wake pattern from a $2 S$ (single vortex shed per side of the oscillating cylinder) mode to a $2 P_{0}$ mode (a vortex pair shed per side of the oscillating cylinder, one of more energetic than the other).

- When $2 \pi U^{*} \approx 5$ (inverse of the Strouhal number of the circular cylinder) the non-dimensional amplitude of oscillation $A^{*}=A / D$ is maximum and the frequency of oscillation is $\omega^{*} \approx 1$. The amplitude of oscillation then decreases slowly with the reduced velocity until $2 \pi U^{*} \approx 8$. In this range the mode of vortex shedding goes from $2 P_{0}$ to $2 P$ (a vortex pair shed per side of the oscillating cylinder). The shedding of vortices is synchronized with the cylinder's motion (lock-in).

- In the range of $8<2 \pi U^{*}<11$ the amplitude of oscillation takes a quite constant value as well as the frequency of oscillations. The mode of vortex shedding is $2 P$. The shedding is also synchronized with the cylinder's motion.

- For $2 \pi U^{*}>11$ synchronization of vortex shedding and cylinder's oscillations is lost (lock-out), i.e. the vortices are shed without any link to the cylinder's position, and the amplitude of oscillation drops abruptly.

The amplitude response shows a somewhat bell-shaped character, which resembles a resonance character. However, this resonance is more complex than the traditional one observed in an linear oscillator 
under a forcing term since it has a remarkably non-linear character. The range of the the lock-in regime (where oscillations are significant) is highly influenced by the reduced mass (or mass ratio), being larger when the reduced mass is lower. In fact, when the reduced mass $m^{*}$ is lower than a certain threshold (known as critical mass), the resonance between the wake and the elastic system occurs for all values of the fluid velocity and both frequencies are locked-in, thus large amplitude oscillations regime is expected for all values of the incident velocity (Govardhan and Williamson, 2002).

Finally, it should be said that effort has been put into modeling satisfactorily VIV dynamics through analytical models in the last 50 years (Gabbai and Benaroya, 2005). In general terms, these models present heuristic and semi-empiric formulations that are introduced appropriately through parameters obtained through experimental data. Their validity are normally limited to a reduced range of the parameters and their capacity of prediction are scarce. One of the main reasons is that the frequency of vortex shedding and the natural frequency of oscillation of the elastic system are of the same order, therefore making it necessary to take into account the interaction occurring between the vortices being shed and the movement of the structure which is a highly non-linear interaction. Therefore, investigations of VIV related phenomena are usually performed through numerical simulations (specially for the lower end of Reynolds numbers) and experimental campaigns (for moderate to high Reynolds numbers).

\subsubsection{Transverse galloping}

The second FIV phenomenon under consideration is an important class of flow-induced oscillatory instability which is appropriately called transverse galloping. Its name is due to Den Hartog because of the visual impression given by its typical low-frequency, high amplitude oscillations. Transverse galloping arises as a result of a self-excited instability of the fluid-elastic system to small displacements of the elastic structure with the expected result that the exciting forces will disappear if oscillations cease. Transverse galloping is a common phenomenon of structures with low structural damping which have specific cross-section geometries prone to galloping such as traffic signs, bridge decks or in marine conduction where organic matter deposit and produce an elliptical shape (Simpson, 1972).

(a)

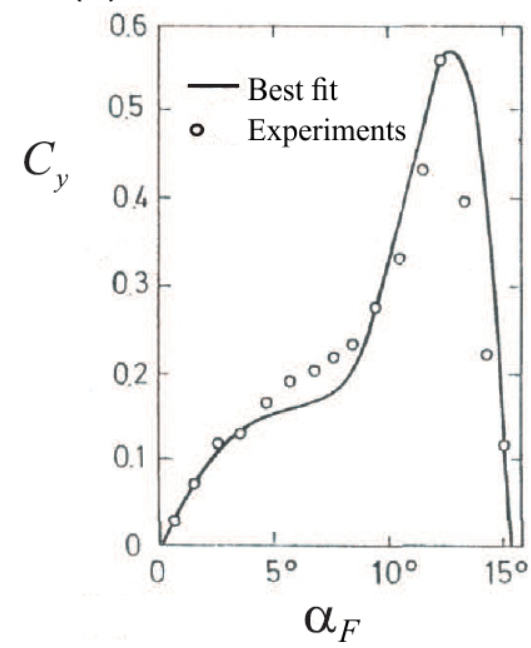

(b)

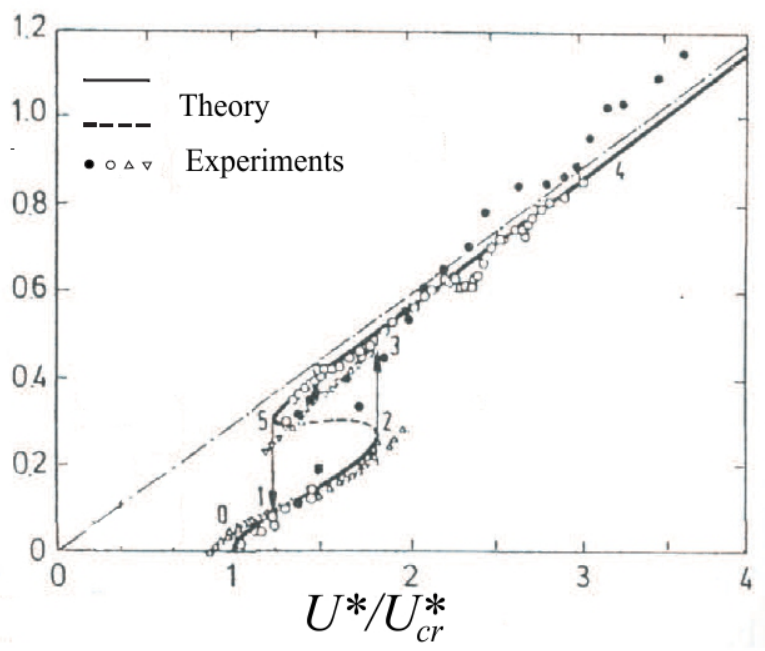

Figure 1.4: (a) Averaged transverse aerodynamic force coefficient, normal to the incident current for a square section prism measured in conditions of low turbulence of the incident current. (b) Transverse galloping response (LCO) of the same prism of square section with different values of mass and damping in a low level turbulence stream. (Parkinson and Smith, 1964).

The canonical example consists of a slender prism with a certain cross-section geometry (namely, 
D-section, square or triangular) elastically-mounted and free to oscillate in the transverse direction immersed in a cross-flow. After a certain velocity has been overcome (usually known as critical galloping velocity), the stabilizing effect of the structural damping is surpassed by the destabilizing effect of the fluid force that appears for small transverse displacements of the prism. Then, a net flux of energy from the flow to the prism appears and the later starts to oscillate transverse to the flow, with increasing amplitude until a Limit Cycle Oscillations (LCO) is reached. Differently from VIV, once the instability goes off, amplitude of oscillations grows with the reduced velocity unlimitedly.

Transverse galloping is a self-induced phenomenon mainly driven by the induced angle of attack (not by vortex shedding), which can only appear for specific cross-sections in which a variation of the induced angle of attack produces a transverse fluid force in the direction of the velocity of oscillation (see Fig. 1.4a). As can be seen in Fig. 1.4b, one notable difference of transverse galloping response compared to that of VIV is that in the former the amplitude of oscillations grows continuously with the reduced velocity without limit. Broadly speaking, cross-sections prone to galloping are those in which:

- There is an asymmetry in the cross-section geometry (not axisymmetric).

- There is a short aft-body (short area of the cross-section behind the detachment points).

- Minimum geometrical area in front of the aft-body. The flow ahead the detachment points have a stabilizing effect.

- The flow around the side facing in the direction of oscillation should be attached, whereas the flow around the opposite face should be completely detached and to maximize the phenomenon there should be no reattachment.

Ding et al. (2015) investigated different cross-sections in order to determine which ones were best suited for energy harvesting from transverse galloping.

In Chapter 4 of the thesis an in-depth description of transverse galloping phenomenology is presented. Also an analytical treatment of the fluid forces is presented as a result of the disparity of timescales between the oscillation and the convective characteristic timescale of the fluid, thus a quasi-steady approximation can be resorted to.

\subsection{Objectives, outline and main results of the thesis}

The overall objective of the thesis is to gain insight into the potential of Vortex-Induced Vibrations and transverse galloping to extract energy efficiently from a fluid flow as a first step of a more ambitious project to design devices to produce clean electrical energy from geophysical flows both in the microscale (say miliWatt) or the macro-scale (kilo/megaWatt). Specific objectives include:

- To see if it is possible to enhance energy transfer from a fluid flow to a spring-mounted circular cylinder by rotating the cylinder appropriately around its axis. If so, to explain why this happens based on the flow field around the oscillating cylinder.

- To see if Lattice Boltzmann Method can be easily implemented for the study of flow-induced vibration problems.

- To see if it is possible to develop mathematical models, of the energy harvesting from transverse galloping full problem (that is, considering the double fluid-solid-electric coupling) maintaining a compromise between simplicity (to make useful a comprehensive analysis) and accuracy. From a practical perspective these models should serve, for example, to suggest optimization laws on any of the part of the whole process (fluid/solid/transducer). 
To carry out these objectives, computational, experimental, and analytical approaches have been followed. As will be shown later in detail, experiments have been carried out at the "Antonio Barrero Ripoll" free surface re-circulating water channel with a spring-mounted rigid circular cylinder with externally imposed rotation around its axis. Laser displacement sensors and accelerometers have been employed to record cylinder's dynamics and Particle Image Velocimetry to measure flow field near the cylinder's surface.

The rest of the thesis is organized as follows:

- In Chapter 2, a numerical simulation of transverse flow-induced vibrations of a rotating circular cylinder is presented. Rotation rate and direction is imposed to be proportional either to the cylinder's position or cylinder's velocity. To our knowledge this is the first time that such rotating law is proposed. A $2 \mathrm{D}$ Lattice Boltzmann method is used to solve the flow dynamics and the cylinder is spring-mounted and free to undergo transverse vibrations. In all cases, nondimensional mass is close to 10, Reynolds number 100, and zero damping is considered, so the analysis is focused on the role of the rotation law and the reduced velocity. Results are presented in terms of steady-state oscillations characterization (say amplitude and frequency), fluid forces on the cylinder, and wake-pattern topology. It is shown how the imposed rotation changes significantly the cylinder's response, fluid forces on it and the flow field in the wake. Depending on the rotation law imposed flow-induced vibrations can be increased or diminished. The former can be of interest for energy harvesting purposes as explained through Eq. 1.5a; whereas the later can be useful to avoid unwanted oscillations. When rotatory law is proportional to the cylinder's position, a galloping-type response has been found. In this case, the cylinder's amplitude can be reasonably well predicted with a quasi-steady theoretical model. For rotation proportional to the cylinder's velocity only vortex-induced-type responses have been found. Part of the content of this chapter has been sent for publication to Journal of Fluids and Structures.

- In Chapter 3, the same problem considered in Chapter 2 is studied experimentally in a freesurface recirculating water channel, with a larger value of the Reynolds number $(R e \approx 5000)$. The flow field around the circular cylinder has been measured through non-intrusive PIV (Particle Image Velocimetry) techniques. Most of the results found in the numerical computations presented in Chapter 2 are now seen experimentally. Part of the content of this chapter has been sent for publication to Physics of Fluids.

- In Chapter 4, the feasibility of transverse galloping as a way to harvest energy efficiently is studied by developing analytical models. In the full problem there is a fluid-solid-electric coupling. The fluid part is modeled by resorting to the quasi-steady hypothesis. First the mechanical-toelectrical transducer is studied. When electromagnetic conversion is considered, two different levels of simplification have been made depending on the comparison between the characteristic electrical and mechanical timescales. The effect of the electrical resistance load on the energy harvested is studied theoretically. For fixed geometry and mechanical parameters, it has been found that there exists an optimal electrical resistance load for each reduced velocity. On the practical side, this result can be helpful to design tracking-point strategies to maximize energy harvesting for variable flow velocity conditions. When piezoelectric means are employed for mechanical-to-electrical conversion, the piezoelectric generator is modeled by an equivalent electrical circuit where power is dissipated at an electrical load resistance. The mathematical model predicts the influence of the main governing parameters: cross-section geometry, mechanical properties, electrical properties of the piezoelectric generator, electrical load, and velocity of the incoming airstream on the electric generation. The model is also adapted to the practical situation in which the galloping oscillator is a cantilevered beam. Because of the engineering interest of this particular case, the model results are compared to some experimental results 
obtained from specialized literature. In view of this comparison, a discussion on the advantages and shortcomings of the proposed model is presented. Secondly, a modification in the galloping harvester is proposed by introducing a secondary mass (dual-mass system). An improvement in the efficiency of energy harvesting with respect to that of the single mass configuration is found when the mechanical properties of the dual-mass system are appropriately chosen. In addition, the dual-mass system promotes a broadening of the values of the incident flow velocities at which the efficiency is kept high. Finally, the possibility to optimize the energy harvesting "from the fluid-flow side", the galloping of a body (D-type cross-section) where rotation is externally imposed around its geometrical center with different laws is studied. The basic idea is that, thanks to the rotation law, the galloping body is always nearly at the same equivalent angle of attack, where transverse fluid force close to its quasi-steady maximum and hence energy transfer is enhanced. A mathematical model based on the quasi-steady hypothesis is developed and compared with computational results obtained with the Latice Boltzmann Method (same code as the one developed for Chapter 2 with different geometry (D-type) and different rotating laws). Agreement between theoretical model and computational results is good. High increasing in energy extraction can be obtained. Part of the results presented in this chapter have been published in the Q1 journals Journal of Fluids and Structure (electromagnetic transducer), Journal of Sound and Vibration (dual-mass galloping system), and sent for publication Journal of Journal of Wind Engineering \& Industrial Aerodynamics (piezoelectric galloping).

- In Chapter 5 open questions and recommendations for future works are briefly discussed.

In this dissertation, each chapter forms a self-contained study, and therefore includes its own detailed Introduction section and Conclusions section. However, we have found it convenient to place all the references together at the end of the dissertation. Also, notation can change slightly between chapters as we have tried to use conventional notation for each phenomena. Some supplementary material (videos and photographs) are available at www.tfslab.es/david

\subsection{Literature production}

During the development of the present thesis, two articles have been published in major journals of related sectors. These are:

- Vicente-Ludlam, D., Barrero-Gil, A., Velazquez, A. (2014). Optimal electromagnetic energy extraction from transverse galloping. Journal of Fluids and Structures, 51, 281-291.

- Vicente-Ludlam, D., Barrero-Gil, A., Velazquez, A. (2015). Enhanced mechanical energy extraction from transverse galloping using a dual mass system. Journal of Sound and Vibration, 339, 290-303.

A collaboration with a fellow $\mathrm{PhD}$ candidate researcher dealing with the effect of cantilevered beams on transverse galloping (some results have indeed been used in Chapter 4, Section 4.4) was published:

- Xu-Xu, J., Vicente-Ludlam, D., Barrero-Gil, A. (2016). Theoretical study of the energy harvesting of a cantilever with attached prism under aeroelastic galloping. European Journal of Mechanics-B/Fluids, 60, 189-195.

Furthermore, three more articles are under revision

- Vicente-Ludlam, D., Barrero-Gil, A., Velazquez, A. Flow-Induced Vibration of a rotating circular cylinder using position and velocity feedback. Journal of Fluids and Structures. 
- Vicente-Ludlam, D., Xu-Xu, J., Barrero-Gil, A., Velazquez, A. Theoretical study of the optimal piezoelectric energy harvester from transverse galloping. Journal of Wind Engineering \& Industrial Aerodynamics.

- Vicente-Ludlam, D., Barrero-Gil, A. Experimental investigation of flow-induced vibrations of a rotating circular cylinder using position and velocity feedback. Physics of Fluids.

As a part of a research and development program of the research group, UPM P120130288, two patents have been filled in which I appear as coauthor:

- Barrero Gil, A., Velázquez, A., Arias, J.R., Prieto Acedo, O., Vicente Ludlam, D., Xu Xu, J. Energy converters and energy conversion systems. EP 2932091,US 20150330358. 


\section{Chapter 2}

\section{Numerical Flow-Induced Vibration of a rotating circular cylinder using position and velocity feedback}

\section{$2.1 \quad$ Introduction}

Flow-Induced Vibrations (FIV) of elastic bodies are not rare. They take place in a large variety of physical systems, such as airplane wings, leaves of trees, long-span bridges, tall buildings, heat exchange devices, clarinet reeds or offshore structures, to name a few. Such FIV can cause severe (even destructive) vibrations as well as useful motions. The fluid flow and the elastic response of the body are coupled, since the fluid force causes the body to deform and, as the body deforms, its orientation to the flow changes and so does the fluid force. The understanding of this fluid-elastic coupling is of interest from both the scientific side and the practical side (to better design elastic systems according to the required requisites for example).

Vortex-Induced Vibration (VIV) is a type of FIV caused by a non-linear resonance phenomenon. For high enough Reynolds number (say, larger than 50), the flow separates from the body surface generating an unsteady broad wake, where large-scale vortices are shed periodically from the body surface which leads to an alternating fluid force on the body. The shedding frequency of the vortices is related to the undisturbed flow speed and the size and shape of the body. When the frequency of the vortex shedding is close enough to the natural frequency of oscillation of the elastic body a closeto-resonance condition is achieved and significant oscillations in the body can appear. The interaction between the oscillating body and the fluid flow is very complex and depends on a large number of parameters related to fluid flow characteristics (say flow velocity, Reynolds numbers, turbulence of the inflow, and so on), structural (mechanical) properties of the body (mass, stiffness and mechanical damping), and geometric (shape of the body, aspect ratio). VIV has much relevance in several branches of mechanical or civil engineering. Recently, the field of interest has been enlarged since it has been considered as a mean to extract useful energy from fluid flows. Bernitsas et al. (2008) developed a device called VIVACE (acronym for Vortex Induced Vibration Aquatic Clean Energy) to extract energy from water currents by oscillations induced by vortex shedding from a spring-mounted circular cylinder. Important efforts have been made in this field, namely, Grouthier et al. (2013), SanchezSanz et al. (2009), Barrero-Gil et al. (2012). There have been other initiatives to efficiently extract energy taking advantage of other Flow-Induced Vibration phenomena, like galloping (Barrero-Gil et al. (2010), Abdelkefi et al. (2012b, 2013b), Vicente-Ludlam et al., 2014), wake galloping (Jung and Woo-Lee, 2011) or flutter (Doaré and Michelin (2011), Singh et al., 2012). 
VIV has been widely investigated through the canonical problem of a spring-mounted rigid circular cylinder under the action of a uniform fluid flow, both experimentally and numerically. Usually, the main interest has been focused to determine the effect of different mechanical and fluid parameters on the VIV response: steady-state oscillations (amplitude and frequency), fluid forces on the cylinder, or flow pattern in the wake. For a detailed review, the reader is referred to Blevins (1990), Sarpkaya (2010), Paidoussis (2011) and Williamson and Govardhan (2004).

The other canonical problem in the field of FIV of bluff bodies is galloping (Parkinson, 1989). It is a motion-induced instability that appears in elastic bluff bodies with certain geometrical shapes (non-axisymmetric cross-sections, like square, D-section, triangular, H-type (Naudascher and Rockwell, 1994) when the velocity of the incident flow exceeds a critical value. Then, a small transverse displacement of the body induces an angle of attack relative to the incoming flow and an asymmetric pressure distribution, so that fluid force appears in the direction of the displacement in such a way that energy is transferred from the flow to the body and oscillatory motion (mainly transverse to the unperturbed flow) develops. Unlike VIV, which occurs only in a certain range of flow velocities and with self-limited amplitude, galloping takes place for any value of the flow velocity higher than the critical value and has a monotonic increase of amplitude with flow velocity. It is mainly driven by the instantaneous angle of attack between the body and the incoming flow and does not necessarily need a synchronization between the oscillations and vortex formation and shedding.

An imposed asymmetry effect in the VIV of a spring-mounted circular cylinder was numerically studied by Bourguet and Jacono (2014). The cylinder was free to oscillate transversely to the flow over a wide range of reduced velocities. In addition, rotation of the cylinder around its axis, with fixed direction of rotation and fixed rotation rate, was imposed. The cylinder was subject to VIV type of vibrations up to rotation rates of $\alpha=4$ ( $\alpha$ is the non-dimensional rotation rate, defined by the ratio of rotational velocity at the cylinder's surface and the unperturbed flow velocity). Oscillations increase with $\alpha$ as well as the region of synchronization where oscillations are significant (synchronization region) up to a non-dimensional rotation rate close to $\alpha=4$. Notoriously, they also reported a $\mathrm{T}+\mathrm{S}$ wake mode for some specific non-dimensional rotation rates and reduced velocity, which had not been previously reported in the literature on non-rotating cylinder in VIV. Seyed-Aghazadeh and ModarresSadeghi (2015) experimentally studied this same problem for Reynolds number between 350-1000, obtaining that the lock-in regime got narrower at high rotation rates and oscillations ceased beyond $\alpha=2.4$. When the cylinder is also let to oscillate in-line Stansby and Rainey (2001) reported large oscillations with amplitudes higher than 10 diameters, with low-frequency galloping-like responses occurring without lock-in.

Another category deals with imposed combined translational and rotational oscillation. Blackburn et al. (1999) were able to generate thrust in quiescent fluid by imposing combined translational and rotational oscillation. Nazarinia et al. (2009a, 2009b) extended this study and experimentally measured the flow around a circular cylinder undergoing imposed combined translational and rotational motion in a free stream obtaining new interesting wake modes. Al-Mdallal (2004), Kocabiyik and Al-Mdallal (2005) and Nazarinia et al. (2009a) showed the possibility of reducing the synchronization region of the cylinder's motion in the near wake through the effect of the phase shift, velocity ratios and motion frequency of the translational and rotational modes.

In the above commented studies, the rotation imposed to the cylinder is not linked to the dynamics of the cylinder, and therefore is not coupled with the result of the Fluid Structure Interaction. In this sense we thought that it could be of interest to link the rotation direction and rotation rate to the cylinder's dynamics, and to see if it could be a way to reduce or enhance the oscillations. In the present study, the rotation of the cylinder is imposed to be proportional to its own position (that is, $\theta=k_{1} y$, where $\theta$ is the rotation angle, $y$ the cylinder's displacement, and $k_{1}$ is a constant) or to be proportional to its velocity of oscillations $\left(\theta=k_{2} \dot{y}\right)$. To study this question, a $2 \mathrm{D}$ numerical Lattice Boltzmann Method is employed. It is investigated how the selected strategy of cylinder's rotation 
(position/velocity), affects the cylinder's response, namely the steady-state amplitude and frequency of oscillations variation with flow velocity, the maximum amplitude of oscillations achieved, how the synchronization regime is modified, the fluid forces on the cylinder, as well as studying the flow pattern in the wake of the cylinder. As it will be shown later in detail, depending of the strategy chosen, it is possible to reduce the oscillations caused by VIV, which may be of interest in practical engineering applications, or enhance the oscillations, which may have implications in the domain of flow energy harvesting for example. The Reynolds number during simulations was 100. As shown in Bourguet and Jacono (2014), at this Reynolds number regime the flow field is 2D and three-dimensional transition does not occur except for relatively high rotation rates, thus, it is considered that a $2 \mathrm{D}$ numerical model is accurate enough.

LBM may present several advantages for simulating FIV problems, due to its local nature and its easy implementation for moving boundaries (no moving meshes are necessary for example). For a detailed review refer to Chen and Doole (1998). However, to our knowledge there are still few numerical FIV studies using LBM. We can cite here Ku and Lin (2005), Mussa et al. (2009), Jiang et al. (2016). In this sense a secondary goal of this study is to further collaborate in the analysis of FIV problems using the LBM method proving that results shed by these methods are reliable.

The rest of the paper is organized as follows, in Section 2.2, the LBM method is described and it is shown how it has been implemented to the particular problem at hand. In addition, the validity of the formulation is demonstrated by comparing with previous results published in the literaure; in Section 2.3, results for the cylinder with prescribed rotatory law proportional to the cylinder's position is presented first. Significant changes in the cylinder's response are found. Depending on the sign of the constant of proportionality between rotation and cylinder's displacement, the response can be diminished or significantly enhanced (even a galloping-type response emerges when the forced rotation overcomes a certain level). Right after the results for the cylinder with a rotation law proportional to the cylinder's velocity is presented. In this case, it is possible to increase or diminish oscillations but the response has always a vortex-induced-type character. As above commented, for certain cases of the rotating law a galloping-type response has been observed. A quasi-steady theoretical model can reasonably predict amplitude of oscillation for these cases. This model is presented in Section 2.4. In Section 2.5, a discussion about the level of actuation required to get the same changes in amplitude's response (with respect to the non-rotating cylinder) for the two rotation laws proposed is made and a comparison of the intensity of the vortices shed is made. Finally, concluding remarks are presented in Section 2.6.

\section{$2.2 \quad$ Numerical Method}

\subsubsection{Fluid-structure model}

Let us consider a spring-mounted circular cylinder immersed in a 2D cross-flow that is parallel to the $x$-axis with an incoming flow velocity $U$ (see Fig. 2.1). The Reynolds number, based on the flow velocity $U$ and the cylinder diameter $D$, has been chosen to be 100 . At $R e=100$ the flow remains two dimensional over all the parameter space investigated, thus the 2D Navier-Stokes equations are considered to be valid to predict the flow dynamics.

The circular cylinder is restricted to move in the $y$ direction (transverse to the incident flow) and is supported with a linear spring with constant stiffness per unit length $k$, viscous damping with constant damping per unit length $c$, and mass per unit length $m$. The cylinder's rotation $(\theta)$ around its own axis is imposed to be either proportional to its displacement $(y)$ or its velocity $(\dot{y})$. The balance between inertia, damping, stiffness and fluid forces gives the following ordinary differential equation for the system: 


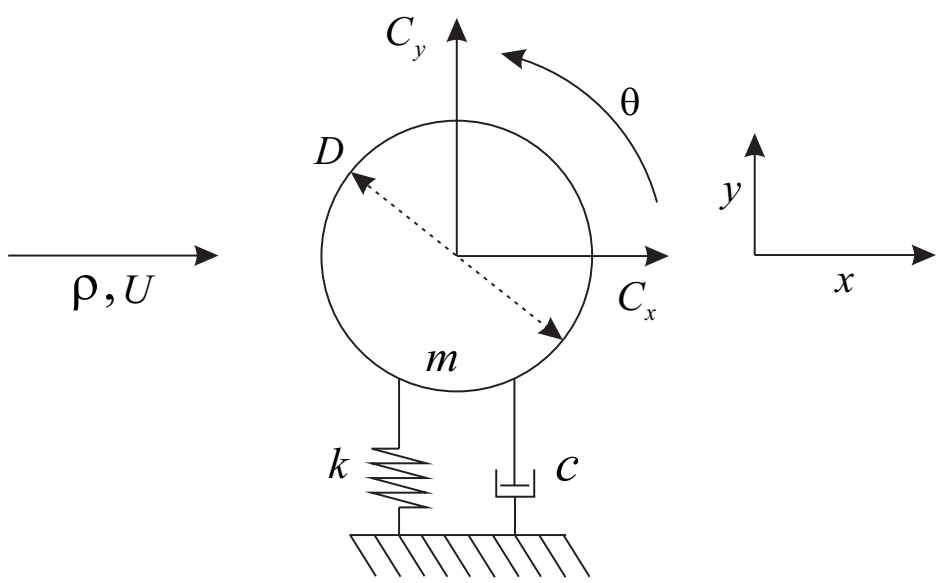

Figure 2.1: Schematics of the rotating spring-mounted circular cylinder.

$$
m \ddot{y}+c \dot{y}+k y=\frac{1}{2} \rho U^{2} D C_{y},
$$

where $y$ denotes the transverse displacement of the prism, $\rho$ is the fluid density, $U$ is the undisturbed velocity of the incident flow, $D$ is the diameter of the circular cylinder, $C_{y}$ is the instantaneous fluid force coefficient in the transverse direction to the incident flow. The rotation has been imposed to be either proportional to its displacement or to its velocity

$$
\theta=k_{1} y \quad \text { or } \quad \theta=k_{2} \dot{y}
$$

where $k_{1}$ and $k_{2}$ are the feedback constants to be imposed.

Introducing non-dimensional variables $Y=y / D, Y^{\prime}=\dot{y} /\left(f_{N} D\right)$ and $t^{*}=f_{N} t$, where $f_{N}=$ $\sqrt{k / m} /(2 \pi)$ it follows

$$
\begin{gathered}
Y^{\prime \prime}+4 \pi \zeta Y^{\prime}+4 \pi^{2} Y=\frac{2 U^{* 2} C_{y}}{\pi m^{*}}, \\
\theta=\tilde{k}_{1} Y \quad \text { or } \quad \theta=\tilde{k}_{2} Y^{\prime},
\end{gathered}
$$

where $m^{*}=4 m /\left(\pi \rho D^{2}\right)$ is the mass ratio, $\zeta=c /\left(4 \pi m f_{N}\right)$ is the damping ratio, $U^{*}=U /\left(f_{N} D\right)$ is the reduced velocity, $\tilde{k}_{1}=D k_{1}$ and $\tilde{k}_{2}=f_{n} D k_{2}$ are the non-dimensional feedback constants and ' stands for differentiation with respect non-dimensional time $t^{*}$.

\subsubsection{Numerical simulation method}

To compute the fluid force in Eq. 2.3a ( say $C_{y}$ ) numerical simulations have been carried out employing the Lattice Boltzman Method, which is a discretization of the Boltzmann equation based on the kinetic theory. Using the Bhatnar-Gross-Krook (BGK) approximation for the collision operator and discretizing, the Lattice Boltzmann Equation is achieved (Higuera, 1989)

$$
f_{i}\left(t+\delta_{t}, \mathbf{x}+\mathbf{e}_{i} \delta_{t}\right)-f_{i}(t, \mathbf{x})=\Omega(t, \mathbf{x})=\frac{1}{\tau}\left(f_{i}^{e q}(t, \mathbf{x})-f_{i}(t, \mathbf{x})\right),
$$

where $\mathbf{e}_{i}$ is the finite direction set, $f_{i}(t, \mathbf{x})$ represents the density distribution of the particles moving in the $\mathbf{e}_{i}$ direction at time $t$ and position $\mathbf{x}, \delta_{t}$ is the discrete time-step, $\tau$ is a relaxation time parameter, and $f_{i}^{e q}$ is the Boltzmann distribution for the particles at equilibrium with macroscopic velocity $\mathbf{u}$

$$
f_{i}^{e q}=w_{i} \rho\left(1+\frac{\mathbf{e}_{i} \cdot \mathbf{u}}{c_{s}^{2}}+\frac{\left|\mathbf{e}_{i} \cdot \mathbf{u}\right|^{2}}{2 c_{s}^{4}}-\frac{\mathbf{u}^{2}}{2 c_{s}^{2}}\right),
$$


where $w_{i}$ is a weighted parameter, $\rho$ and $\mathbf{u}$ are, respectively, the macroscopic density and velocity of the fluid, and $c_{s}$ is the lattice sound speed. For our numerical simulations the D2Q9 model has been chosen to use (see Fig 2.3a). Accordingly

$$
\mathbf{e}_{i}=\left\{\begin{array}{lr}
0 & i=0 \\
c(\cos ((i-1) \pi / 4), \sin ((i-1) \pi / 4)) & i=1-4 \\
\sqrt{(2) c(\cos ((i-1) \pi / 4), \sin ((i-1) \pi / 4))} & i=5-8
\end{array}\right.
$$

where $c=\partial x / \partial t$ and the weighted parameter $w_{i}$ is given as

$$
w_{i}=\left\{\begin{array}{lr}
4 / 9 & i=0 \\
1 / 9 & i=1-4 \\
1 / 36 & i=5-8
\end{array}\right.
$$

The macroscopic variables such as the fluid density and the momentum are related to $f_{i}$ as

$$
\rho=\sum_{i} f_{i}, \quad \rho \mathbf{u}=\sum_{i} \mathbf{e}_{i} f_{i} .
$$

The discrete time-step $\delta_{t}$ is set equal to unity as well as the lattice spacing $\delta_{x}$ (thus $c_{s}=1 / \sqrt{3}$ and $c=1$ ). The Lattice Boltzmann computer algorithm usually employed consists of 2 steps :

$$
\begin{aligned}
\text { Collision: } & =\tilde{f}_{i}(t+1, \mathbf{x})=f_{i}(t, \mathbf{x})+\frac{1}{\tau}\left(f_{i}^{e q}(t, \mathbf{x})-f_{i}(t, \mathbf{x})\right), \\
\text { Streaming: } & =f_{i}\left(t+1, \mathbf{x}+\mathbf{e}_{i}\right)=\tilde{f}_{i}(t+1, \mathbf{x}) .
\end{aligned}
$$

In the collision step, the values of the distribution function of the particles are updated at time step $t+1$, and then in the streaming step the updated distribution functions are transferred to their neighboring cells $\mathbf{x}+\mathbf{e}_{i}$.

Applying the Chapman-Enskog expansion in the discrete Lattice Boltzmann Equation the NavierStokes equations can be recovered for small Mach values. Then, the relaxation parameter can be related to the viscosity of the fluid as

$$
\nu=(2 \tau-1) / 6 .
$$

The viscosity has to be positive, so it requires $\tau>1 / 2$; however, due to stability reasons, $\tau$ has to be higher than a certain threshold. Stability issues have been thoroughly investigated (see Mei et al., 1999) and for the present article, $\tau$ has been fixed to 0.56 yielding a value of numerical viscosity $\nu=0.02$.

\subsubsection{Computational domain and boundary conditions}

The computational domain and boundary conditions are shown in Fig. 2.2. The diameter $D$ of the cylinder has 40 lattice points (observe that the number of lattice point for the diameter of the cylinder $N_{D}$ is linked to the Reynolds number, $\nu$ and inflow velocity $U$, since $N_{D}=\nu \operatorname{Re} / U$ ) and the computational domain chosen is $40 D \times 30 D$, giving a size of $1600 \times 1200$ lattice points.

Regarding the boundary conditions, the left boundary is set to be a velocity specified inflow boundary condition with constant velocity profile $U$ (Zou and He, 1997). To simulate the incompressible condition, the Mach number must be low enough, to this end, the inflow velocity $U$ is set to 0.05 . The rest of the outer computational boundaries are considered outflows with extrapolation boundary (Yu et al., 2003). For the boundary conditions on the cylinder, a no-slip boundary condition has been applied on the cylinder surface with an interpolated bounce-back scheme as developed by Mei et al. (1999), which is second order accurate. Following Mei et al. (1999), the postcollision distribution function at the boundary point $\mathbf{x}_{b}$ necessary to determine the forces on the circular cylinder is: 


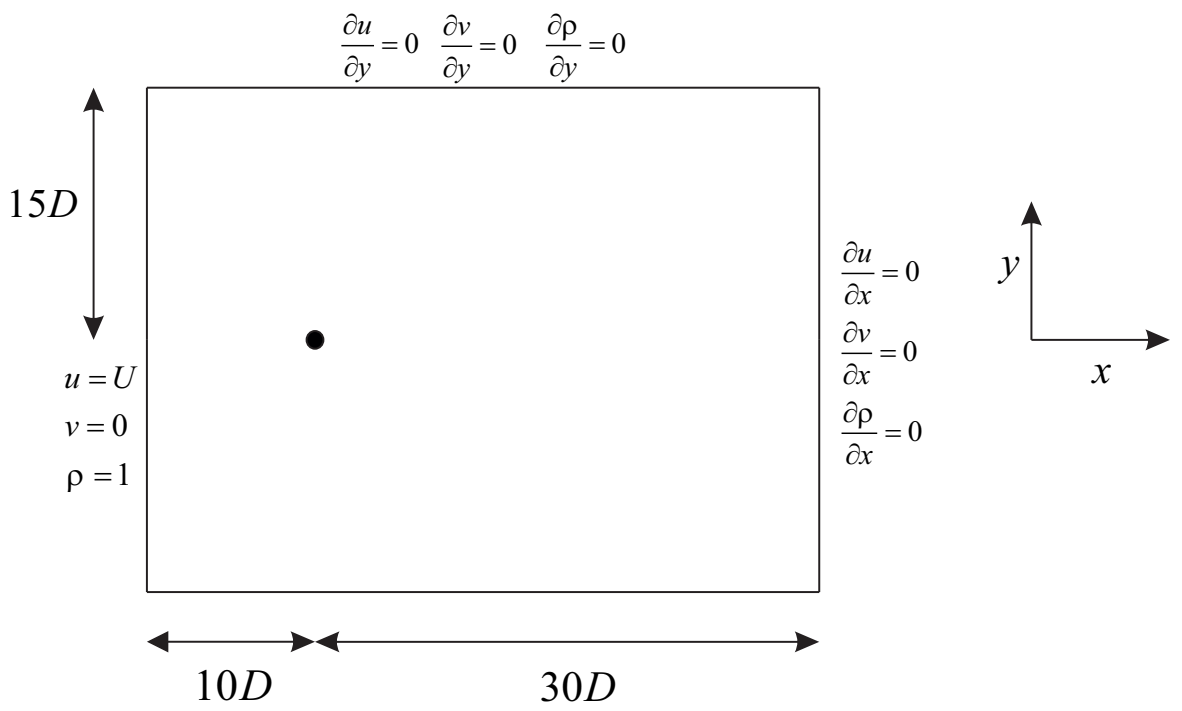

Figure 2.2: Schematics of the computational domain and boundary conditions.

(a)

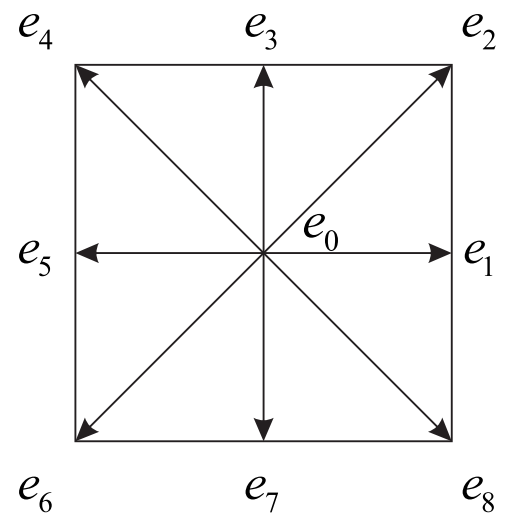

(b)

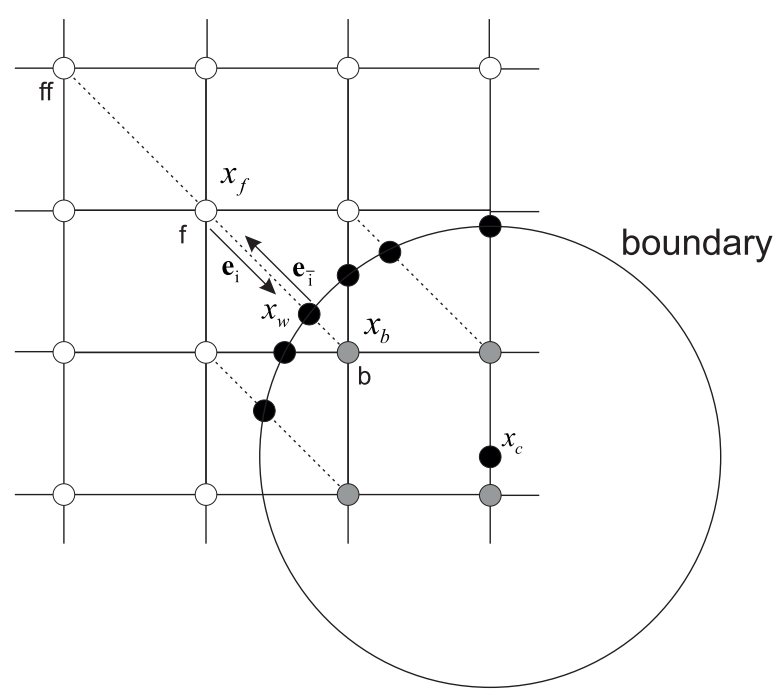

Figure 2.3: (a) D2Q9 lattice model and (b) schematics of the moving boundary condition treatment of the circular cylinder.

$$
\tilde{f}_{\bar{i}}\left(t, \mathbf{x}_{b}\right)=\tilde{f}_{i}\left(t, \mathbf{x}_{f}\right)-\chi\left(\tilde{f}_{i}\left(t, \mathbf{x}_{f}\right)-f_{i}^{e q}\left(t, \mathbf{x}_{b}\right)\right)+w_{i} \rho \frac{3}{c^{2}} \mathbf{e}_{\bar{i}} \cdot\left(\mathbf{u}_{b f}-\mathbf{u}_{f}-2 \mathbf{u}_{w}\right),
$$

and

$$
\begin{gathered}
\mathbf{u}_{b f}=\mathbf{u}_{f f}=\mathbf{u}_{f}\left(\mathbf{x}_{f}+\mathbf{e}_{\bar{i}}, t\right), \quad \chi=\frac{(2 \Delta-1)}{\tau-2}, \quad 0 \leq \Delta<\frac{1}{2}, \\
\mathbf{u}_{b f}=\frac{1}{2 \Delta}(2 \Delta-3) \mathbf{u}_{f}+\frac{3}{2 \Delta} \mathbf{u}_{w}, \quad \chi=\frac{(2 \Delta-1)}{\tau+1 / 2}, \quad \frac{1}{2} \leq \Delta<1 .
\end{gathered}
$$

Here, $\mathbf{x}_{b}$ is the position of the boundary node, $\mathbf{x}_{w}$ is the wall position and $\Delta=\left\|\mathbf{x}_{f}-\mathbf{x}_{w}\right\| /\left\|\mathbf{x}_{f}-\mathbf{x}_{b}\right\|$ is the distance to the wall from the fluid node (see Fig. 2.3b). $\tilde{f}_{i}$ is the post-collision distribution function, $f_{\bar{i}}$ denotes the distribution functions of the velocity $\mathbf{e}_{\bar{i}} \equiv-\mathbf{e}_{i}$, and $\mathbf{u}_{w}$ is the velocity of the moving wall. Noticeably, the velocity to be imposed on the cylinder's contour $\left(\mathbf{u}_{w}\right)$ has to be the sum of the vertical velocity $\dot{y}$ and the tangential velocity due to the rotation of the cylinder. Thus the last term in Eq. 2.11 is an additional forcing term that accounts for the momentum exchange caused by 
the wall's movement (see for example Lallemand and Luo, 2003).

As the cylinder moves, some lattice-points inside the cylinder become fluid node. In this case, the distribution function of any of these appearing fluid nodes is set at the equilibrium function with the cylinder's velocity and the density of the average value of the neighboring fluid nodes.

\subsubsection{Force evaluation}

In LBM, the fluid forces on a specified boundary are usually evaluated using two different methods: the traditional method (Inamuro et al., 2000), which uses the macrovariables of the fluid in the boundary, and the Momentum Exchange Algorithm (MEA) method (Caiazzo and Junk, 2008) which uses the distribution functions on the boundary directly to calculate the forces acting on it. Some advantages of the Momentum Exchange Algorithm include that it is easy to implement and its accuracy and efficiency have been thoroughly investigated. Thus, in this study the MEA method was implemented and the fluid force on the cylinder is computed as

$$
\mathbf{F}=\sum_{\left(\mathbf{x}_{b}, i\right) \subset B} \mathbf{e}_{i}\left(\tilde{f}_{\bar{i}}\left(t+1, \mathbf{x}_{b}\right)+\tilde{f}_{i}\left(t+1, \mathbf{x}_{f}\right)\right)
$$

where $B$ is the cylinder's surface.

\subsubsection{Model validation}

In order to validate the numerical method implemented, it is compared to previous studies available in the literature. In all cases, the Reynolds number was 100 and the lattice resolution was $1600 \times 1200$ (with the cylinder's diameter being 40 lattice points). The first validation of the method is made with the flow past a circular cylinder at rest. The Strouhal number $\mathrm{St}=f_{\mathrm{St}} D / U$, (being $f_{\mathrm{St}}$ the frequency of vortex shedding), the time averaged in-line force coefficient and the maximum transverse fluid force coefficient are computed and compared to previous results published in the literature. As can be noted, with our LBM implementation, the variables considered agree reasonably well with previous studies.

\begin{tabular}{lccc} 
Reference Papers & St & $\bar{C}_{x}$ & $\left(C_{y}\right)_{\max }$ \\
\hline Shiels et al. (2001) & 0.167 & 1.33 & 0.30 \\
Stojković et al. (2002) & 0.165 & 1.34 & 0.33 \\
Shen et al. (2009) & 0.166 & 1.38 & 0.33 \\
Bourguet and Jacono (2014) & 0.164 & 1.32 & 0.32 \\
Present Study & 0.167 & 1.33 & 0.32
\end{tabular}

Table 2.1: Comparison of Strouhal number, in-line average force coefficient and maximum transverse force coefficient for a static cylinder at $R e=100$.

The second validation corresponds to the case of a spring-mounted circular cylinder (non-rotatory) in a cross-flow. The mass ratio $m^{*}$ was 12.7 and zero damping was considered. Fig. 2.4 shows the steady-state normalized amplitude of oscillation variation with the reduced velocity. Results from Shiels et al. (2001) are shown for comparison. Results agree reasonably well; the maximum amplitude obtained with our numerical LBM model is $A^{*}=0.56$ which complies reasonably well with the maximum amplitude of $A^{*}=0.58$ in Shiels et al. (2001).

Finally, the case of a rigidly mounted cylinder rotating at a constant rate is considered. The time averaged in-line $\left(\bar{C}_{x}\right)$ and transverse $\left(\bar{C}_{y}\right)$ fluid force coefficients are shown in Fig. 2.5 as a function 


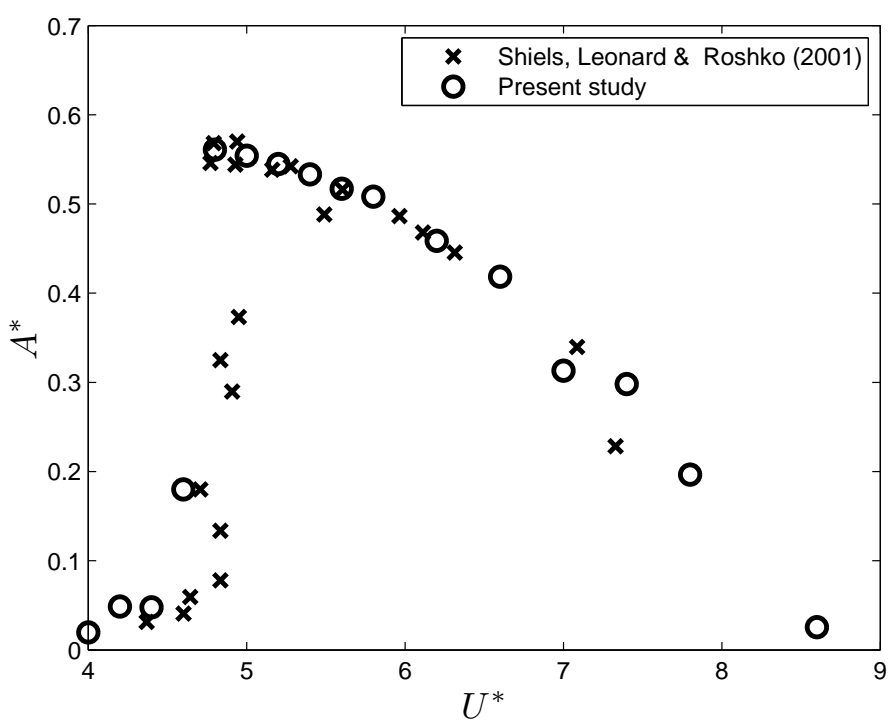

Figure 2.4: Comparison of a VIV amplitude curve for $m^{*}=12.7, \zeta=0$ at $\operatorname{Re}=100$ for Shiels et al. (2001) and the present study.

of the dimensionless rotating rate $\alpha=\dot{\theta} D / 2 U$. Results from Bourguet and Jacono (2014) are also shown for comparison purposes. Good agreement can be observed.
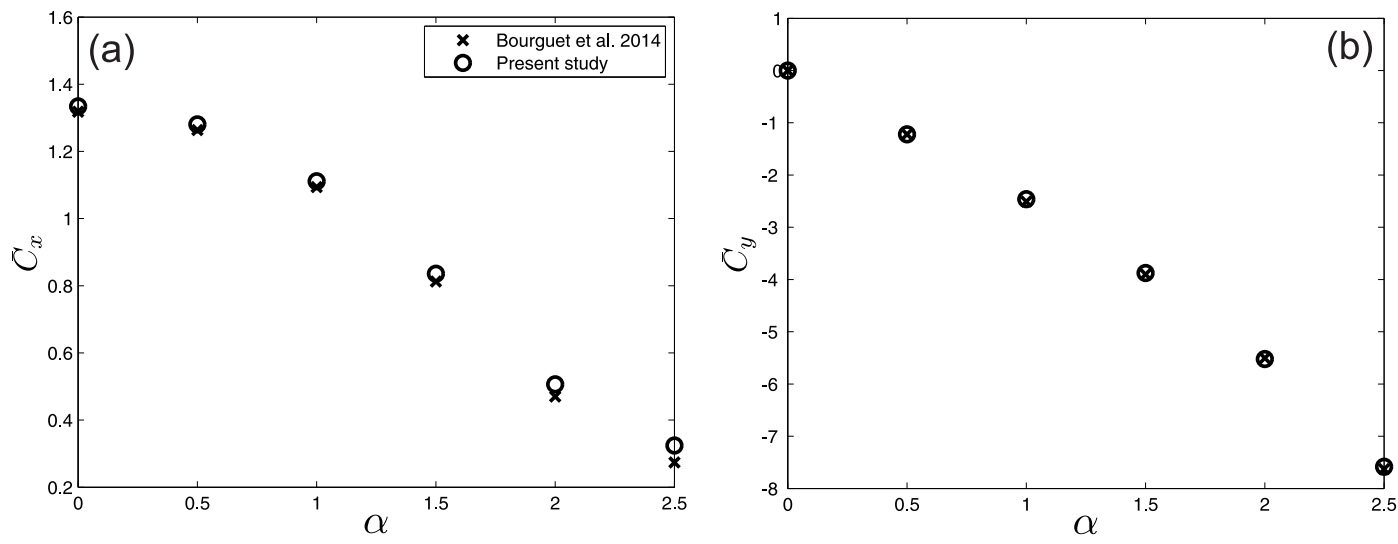

Figure 2.5: (a) In-line and (b) transverse mean force coefficients for a fixed rotating cylinder as a function of the rotation rate at $\mathrm{Re}=100$. Results from Bourguet and Jacono (2014) are used as reference.

\section{$2.3 \quad$ Results}

As seen in Eqs. 2.3a and 2.3b the problem under consideration is governed by the following dimensionless parameters: $m^{*}, \zeta$, the reduced velocity $U^{*}$, the parameter related to the cylinder rotation (either $\tilde{k}_{1}$ or $\tilde{k}_{2}$ ), as well as the Reynolds number. In all computations the Reynolds number was fixed to 100 , the mass ratio 12.7 , and zero damping was considered. Therefore, the analysis focuses on the parametric study of the behavior of the cylinder's response with the reduced velocity $U^{*}$ and the rotation law $\left(\tilde{k}_{1}\right.$ or $\left.\tilde{k}_{2}\right)$. For each configuration (i.e. when $U^{*}$ and $\tilde{k}_{1}$ (or $\tilde{k}_{2}$ ) are fixed), the steady-state cylinder's dynamics (amplitude and frequency of oscillation) was studied as well as the transverse fluid force on the cylinder. Also, wake structure of the flow around the cylinder was analyzed. 
$\tilde{k}_{1}$ and $\tilde{k}_{2}$ were modified with the only limitation that the maximum velocity should be kept approximately under 0.1 to limit compressibility effects.

\subsubsection{Rotation proportional to cylinder's displacement $\left(\theta=\tilde{k}_{1} Y\right)$}

In first place, the response of a circular cylinder undergoing VIV will be modified by rotating the cylinder proportionally to its own transverse displacement. It will be sought if the strategy proposed is able of either enhancing or reducing VIV effects as well as modifying the flow field and the wake pattern appearing behind the cylinder.

$$
\theta=\tilde{k}_{1} Y
$$

where $\tilde{k}_{1}$ is a proportional constant to be modified.

\subsubsection{Cylinder dynamics}
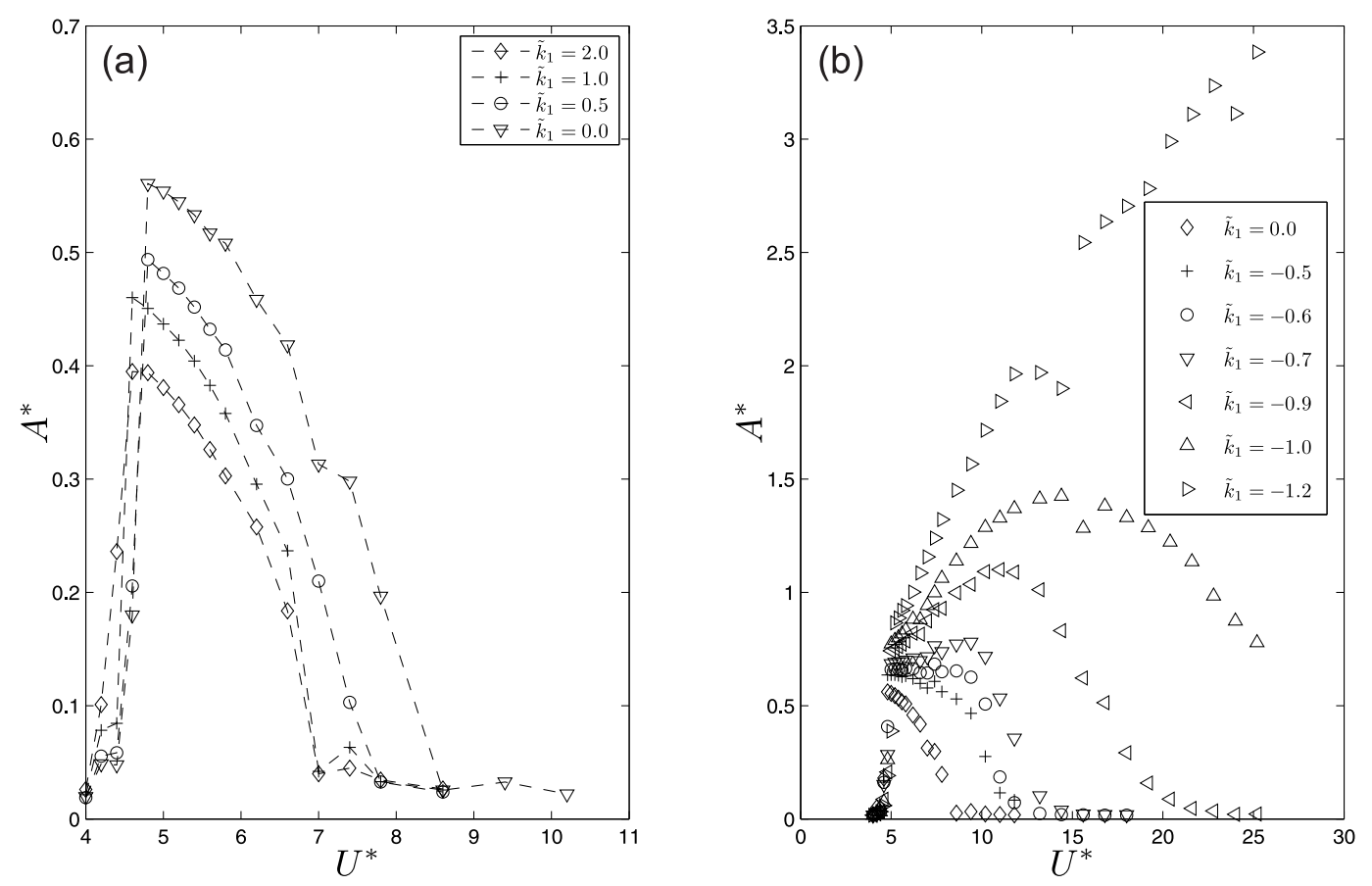

Figure 2.6: (a) Maximum non-dimensional amplitude oscillation as a function of the reduced velocity for positive values of the rotating parameter $\tilde{k}_{1}$ and (b) for negative values of $\tilde{k}_{1}$.

The oscillation of the cylinder exhibits a periodic behavior, being almost purely sinusoidal. Due to the character of the rotation imposed, symmetry is not broken and oscillations take place around the equilibrium position $(Y=0)$ for all values of $U^{*}$ and $\tilde{k}_{1}$. Thus, the cylinder's response can be described by its main frequency of oscillation $f^{*}=f / f_{N}$ and the dimensionless amplitude $A^{*}=A / D$ of the cylinder's steady-state oscillations.

Concerning the amplitude's response, the VIV curve $\left(\tilde{k}_{1}=0\right)$, for the number of Reynolds considered $(R e=100)$, is composed of two branches. Govardhan and Williamson (2006) determined that for zero damping, for Reynolds number over 600, the amplitude curve was composed of three branches; the initial, upper and lower branch. For lower Reynolds number, the upper branch does not appear. As can be seen from Fig. 2.6a, the transition between the initial and lower branch occurs at a reduced 

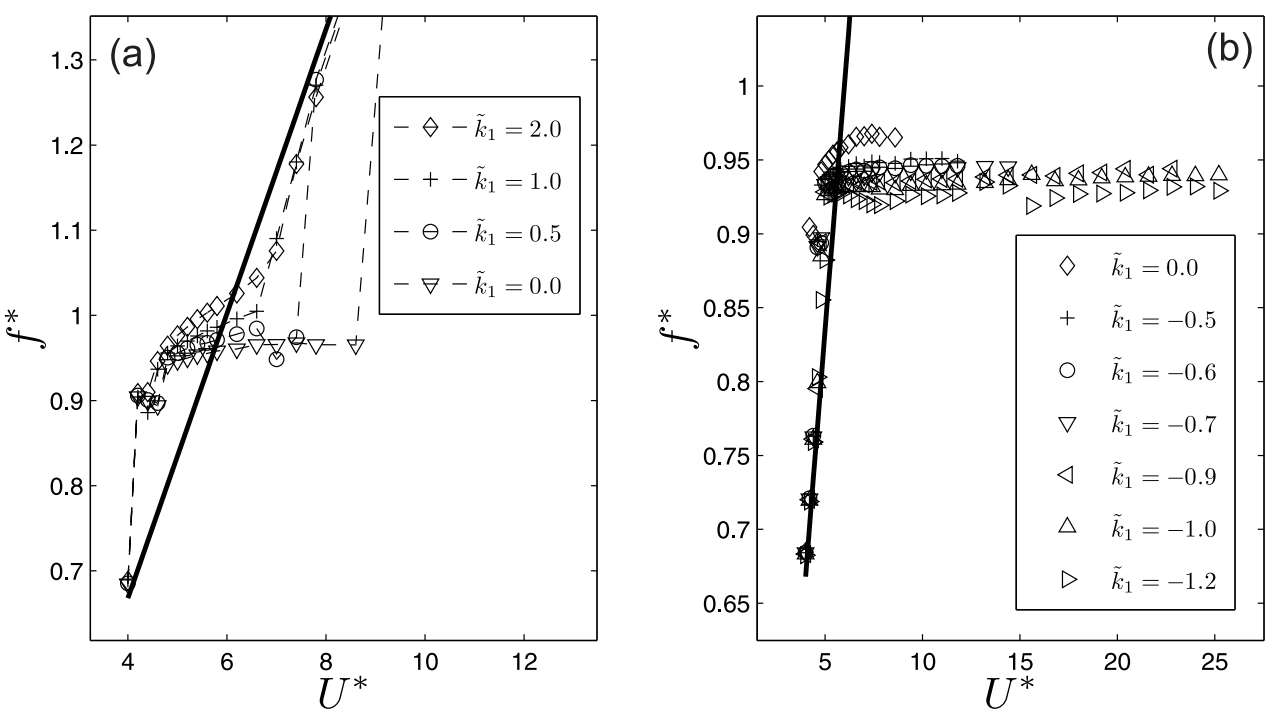

Figure 2.7: (a) Oscillating frequency of the cylinder as a function of the reduced velocity for positive values of the rotating parameter $\tilde{k}_{1}$ and (b) for negative values of $\tilde{k}_{1}$. Strouhal law is shown in bold line.

velocity of approximately $U^{*}=4.8$ with a maximum amplitude of $A^{*}=0.56$. For positive rotation parameter, $\tilde{k}_{1}>0$, the transition between the initial and lower branch is slightly anticipated. The maximum amplitude occurs right after the transition for all positive values of $\tilde{k}_{1}$ and it is importantly diminished. The reduced velocities at which the cylinder undergoes large amplitude oscillations (range of synchronization) is reduced from the pure VIV curve. For example, for $\tilde{k}_{1}=2$ the maximum value of $A^{*}$ has been reduced from 0.56 (for $\tilde{k}_{1}=0$ ) to 0.4 and the range of synchronization (say $A^{*}>0.1$ ) is reduced from $5<U^{*}<8.5$ to $5<U^{*}<7$.

For negative values of $\tilde{k}_{1}$, the picture is quite different (see Fig. 2.6b). Two different responses can be observed regarding the value of $\tilde{k}_{1}$. For $-1<\tilde{k}_{1}<0$, the response amplitude exhibit in all cases a bell-shaped evolution as a function of the reduced velocity which resembles the classical VIV response. This suggests that similar to VIV, the vibrations occur under a wake-body synchronization mechanism. As $\tilde{k}_{1}$ becomes more negative oscillations are more intense, the range of synchronization is enlarged, and the reduced velocity at which maximum amplitude, $A_{\text {max }}^{*}$, is achieved is delayed. For example for $\tilde{k}_{1}=-0.9, A_{\text {max }}^{*}=1.35, U^{*}\left(A_{\text {max }}^{*}\right)=14$, and the synchronization region takes place in the range $5<U^{*}<20$. A different behavior has been found when $\tilde{k}_{1}<-1$. As can be observed, for $\tilde{k}_{1}=-1.2$, just after the initial-to-lower transition $A^{*}$ seems to adopt a quasi-linear dependence with the reduced velocity, which resembles the dependence of galloping-type instability where motioninduced forces are predominant, in contrast to VIV where fluid forces are mainly driven by vortex shedding. This point will be further investigated in the following section.

In Fig. 2.7, the normalized frequency of oscillations $f^{*}$ is plotted as a function of the reduced velocity for different values of $\tilde{k}_{1}$. Also, the Strouhal law is given for comparison in bold solid line $\left(f_{\mathrm{St}}^{*}=\mathrm{St} D / U / f_{N}\right)$ which gives the vortex emission frequency for a cylinder at rest. For $\tilde{k}_{1}=0$, at the initial branch $f^{*}$ is lowered from its natural frequency increasing rapidly with $U^{*}$. After the initial-to-lower transition, $f^{*}$ takes an approximately fixed value close to its natural frequency leading to a lock-in condition. For $\tilde{k}_{1}>0$ (Fig. 2.7a), $f^{*}$ has a bigger dependence with $U^{*}$ after the transition, increasing its value. On the other hand, for $\tilde{k}_{1}<0$ (Fig. 2.7b) the frequency of oscillations is slightly reduced and it achieves a lock-in condition in the sense that after the initial-to-lower transition $f^{*}$ kept almost constant in the lower branch undergoing important oscillations for larger regions of $U^{*}$. 


\subsubsection{Flow field and fluid forces}

In addition to the dynamics response of the cylinder, the vortex shedding structure as well as the characteristics of the fluid forces appearing on the cylinder is investigated. This helps to obtain more information about the synchronization region as well as to determine if there are different mechanisms of excitation, in other words, to determine if the oscillations are driven under a wakebody resonance (VIV-type) or under a motion-induced vibration (galloping-type). In this section, the most relevant wake patterns that appear for different values of $\tilde{k}_{1}$ are presented, paying special attention to synchronization between wake pattern and oscillation. All flow fields are given for the cylinder at the equilibrium point $(Y=0)$ and positive velocity $\left(Y^{\prime}>0\right)$.

Regarding the transverse fluid forces appearing on the circular cylinder, as shown by Govardhan and Williamson (2000), the total transverse fluid force $F_{y}$ on the cylinder can be conveniently split into a "potential-term" $F_{p}$ and a "vortex-associated" term $F_{v}$ :

$$
F_{y}(t)=F_{p}(t)+F_{v}(t)
$$

The potential added-mass force is the fraction of the fluid force exerted on the cylinder that emerges as a result of the acceleration of the body which deflects the surrounding fluid as it moves through it. Evaluating it through the hypothesis of potential flow (Brennen, 1982), the potential added-mass for a circular cylinder is

$$
F_{p}(t)=-\frac{\pi \rho D^{2}}{4} \ddot{y}(t)
$$

where $\pi \rho D^{2} / 4$ is the displaced fluid mass by the circular cylinder and $\ddot{y}$ is the transverse acceleration of the circular cylinder. Normalizing the potential added-mass force one obtains

$$
C_{p}\left(t^{*}\right)=-\frac{\pi}{2} \frac{Y^{\prime \prime}\left(t^{*}\right)}{U^{* 2}}
$$

Note, that the potential-term force coefficient $C_{p}$ is in phase with minus the cylinder's acceleration (in phase with the cylinder's position for sinusoidal oscillations) which is equivalent to an added inertia acting on the body. Then, the "vortex-associated" force coefficient is given by

$$
C_{v}\left(t^{*}\right)=C_{y}\left(t^{*}\right)+\frac{\pi}{2} \frac{Y^{\prime \prime}\left(t^{*}\right)}{U^{* 2}}
$$

This decomposition of the fluid forces can be understood to be a matter of convenience but it is very useful because the vortex force is related to vortex dynamics, and to the convection of vorticity, therefore changes in the vortex force would necessarily correspond to changes in the process of vortex formation. The variation of the phase of $C_{v}$ (and $C_{y}$ ) with respect to the cylinder's displacement (when applicable) will be considered. Also, it will be of interest to study the frequency of the vortexassociated term $f_{v}^{*}=f_{v} / f_{N}$ and compare it to $f^{*}$ to determine the synchronization region, which takes place when $f_{v}^{*} \simeq f^{*}$.

In Fig. 2.8a, the normalized amplitude of oscillation $A^{*}$ for the cylinder undergoing non-rotating VIV is shown (which will be used as a basis to compare with), as well as the vorticity field at four selected values of the reduced velocity $U^{*}$. As previously shown by Singh and Mittal (2005) and in Shiels et al. (2001), and found also here, the response is composed of two branches, the initial and the lower branch, with a jump between them. The wake only shows a $2 \mathrm{~S}$ mode with distinctively different wake patterns depending on $A^{*}$. In this wake mode, during each vortex-shedding cycle, a single vortex is alternatively shed from each side of the cylinder. For those values of $U^{*}$ where the cylinder undergoes high-amplitude oscillations, the vortices in the wake coalesce giving rise in the $\mathrm{C}(2 \mathrm{~S})$ mode (II), in which the vortices coalesce downstream. In Figs. 2.9a- 2.9c, the normalized 


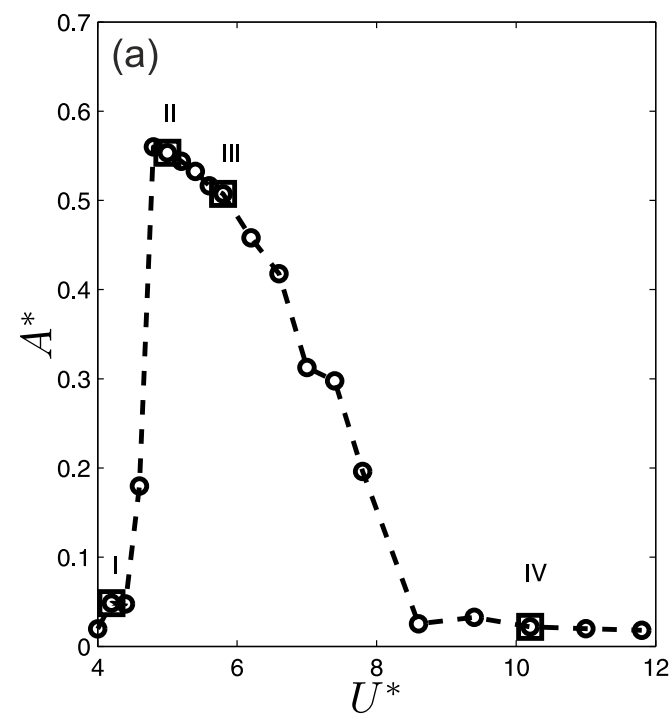

(b)

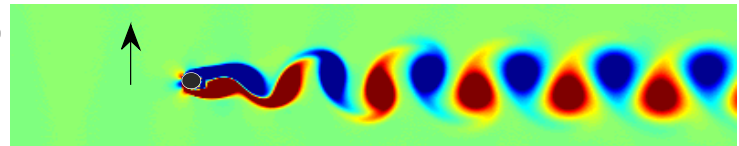

II)

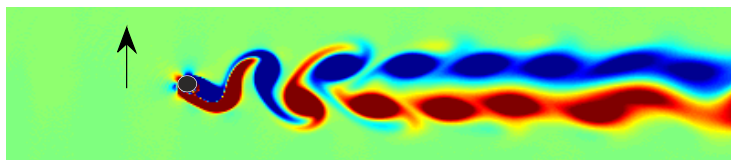

III)

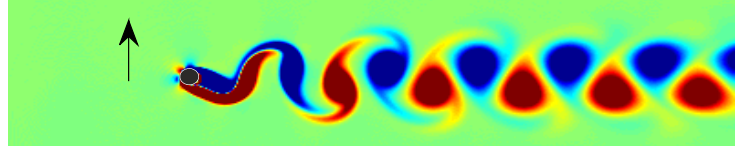

IV)

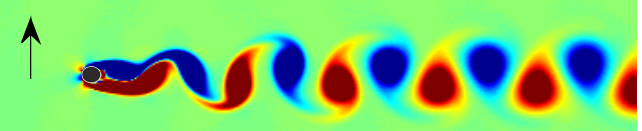

Figure 2.8: (a) Non-dimensional amplitude of oscillation and (b) vorticity field at four different values of the reduced velocity for a rotating parameter of $\tilde{k}_{1}=0$.

(a)

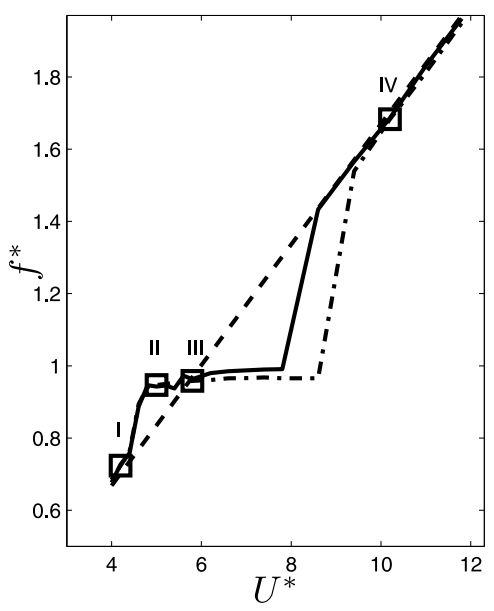

(b)

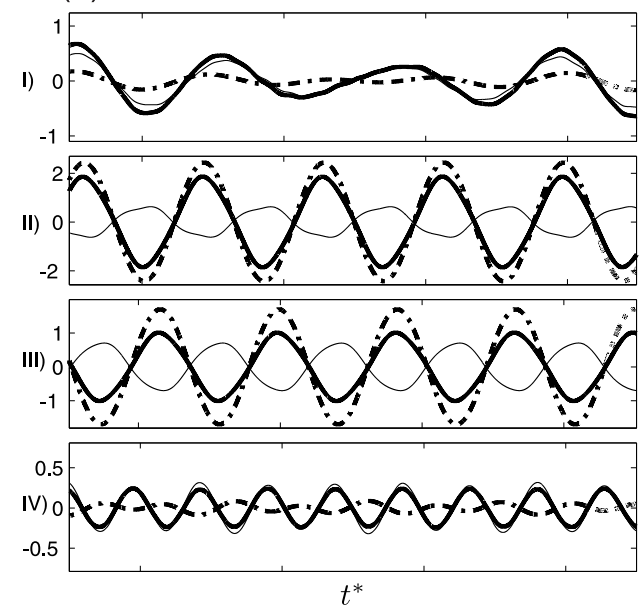

(c)

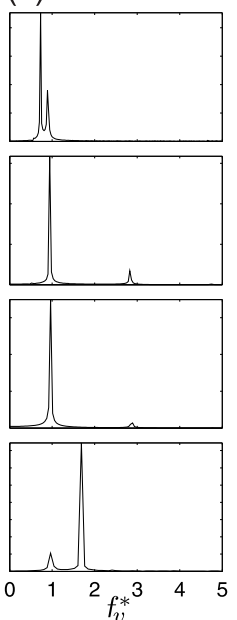

Figure 2.9: (a) Normalized frequency of oscillation $f^{*}$ (dashed line) and normalized frequency of vortex emission $f_{v}^{*}$ (solid line), (b) total transverse force coefficient $C_{y}$ (bold solid line), potential force coefficient $C_{p}$ (dashed line) and vortex force coefficient $C_{v}$ (solid line) and (c) FFT of the normalized vortex force coefficient $C_{v}$ at four values of the reduced velocity for a rotating parameter of $\tilde{k}_{1}=0$.

frequency of oscillation $\left(f^{*}\right)$ and vortex shedding $\left(f_{v}^{*}\right)$ are plotted as a function of $U^{*}$. As it can be seen, in the initial branch (I) the frequency of oscillation is diminished importantly from its natural frequency; as $U^{*}$ increases, so does $f^{*}$ up to a value close to 1 where it remains fairly constant while the cylinder undergoes significant oscillations. At $U^{*} \simeq 8$, the synchronization region finishes, $A^{*}$ is practically zero and both $f^{*}$ and $f_{v}^{*}$ are equal to that given by the Strouhal law (where the frequency of vortex emission at rest is $\left.f_{\mathrm{St}}^{*}=\mathrm{St} D / U / f_{N}\right)$. Fig. $2.9 \mathrm{~b}$, shows the time evolution of the fluid force coefficients $\left(C_{y}, C_{p}\right.$ and $\left.C_{v}\right)$ at selected levels of $U^{*}$. For the initial branch (point marked as I), all $C_{y}, C_{p}$ and $C_{v}$ are all in phase with the cylinder's displacement. After the initial-to-lower jump $C_{v}$ is out of phase with the cylinder's displacement and nearly jumps $180^{\circ}$ (I-II). This result is similar to the one obtained by Govardhan and Williamson (2000), where $C_{v}$ underwent a phase change of $180^{\circ}$ 
in the jump between the initial and upper branch.

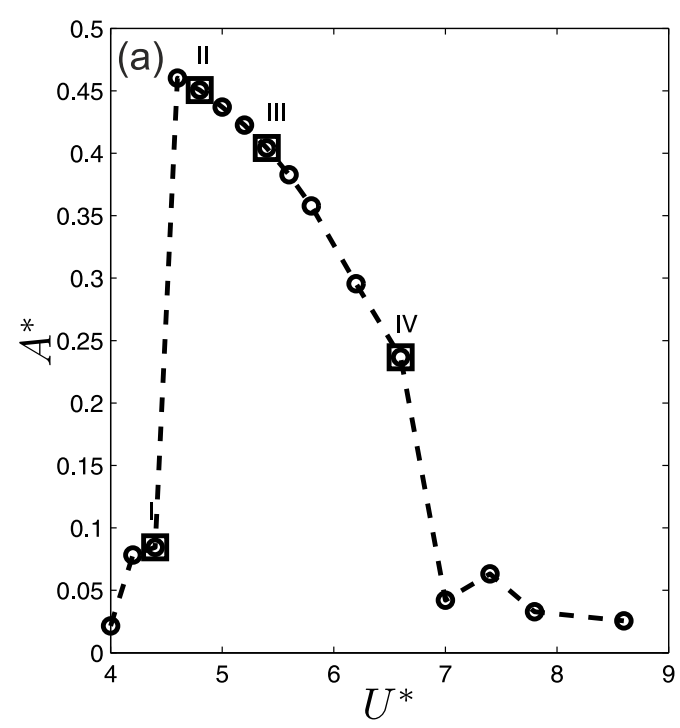

(b)

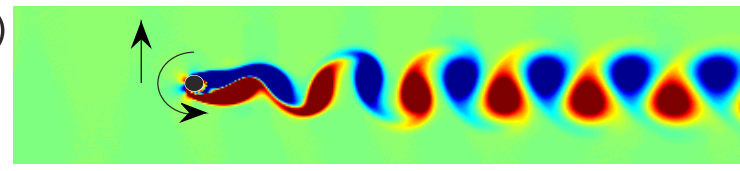

II)

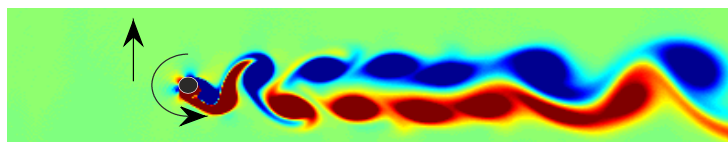

III)
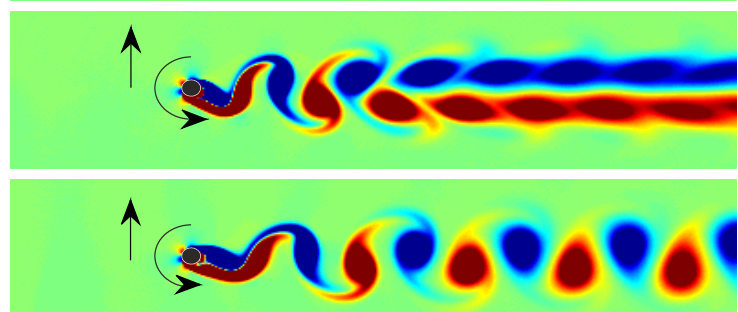

Figure 2.10: (a) Non-dimensional amplitude of oscillation and (b) vorticity field at four different values of the reduced velocity for a rotating parameter of $\tilde{k}_{1}=1.0$.

(a)

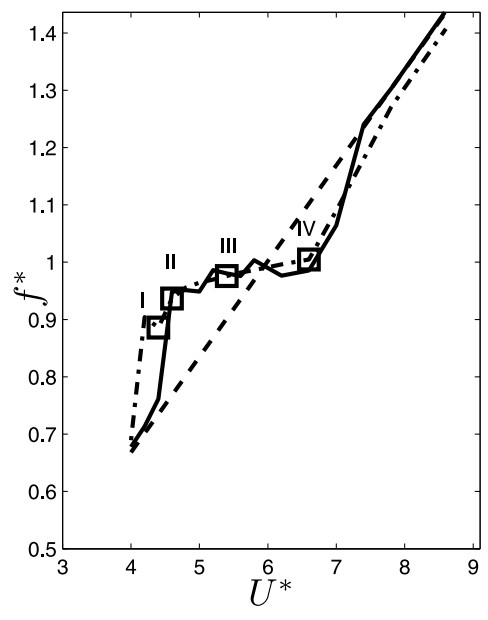

(b)

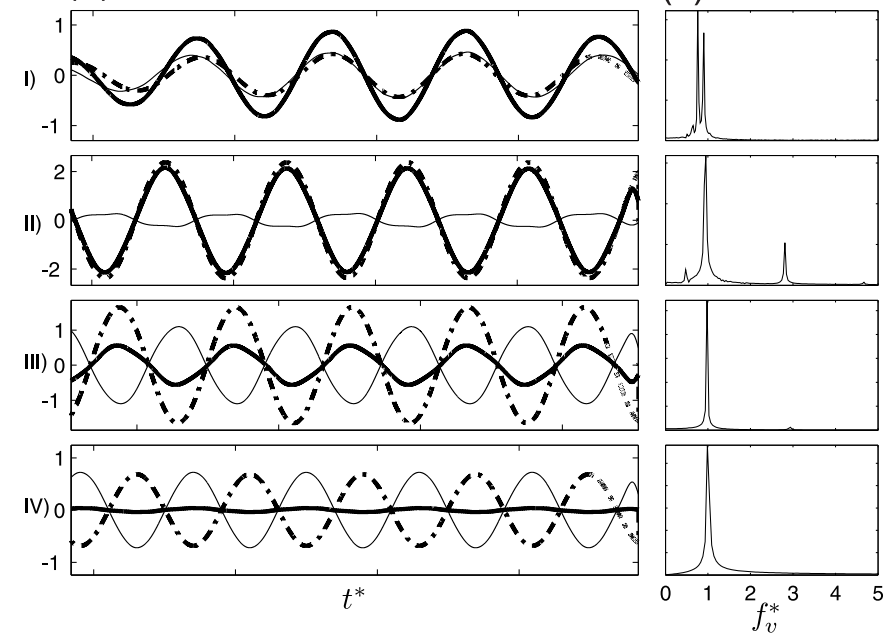

Figure 2.11: (a) Normalized frequency of oscillation $f^{*}$ (dashed line) and normalized frequency of vortex emission $f_{v}^{*}$ (solid line), (b) total transverse force coefficient $C_{y}$ (bold solid line), potential force coefficient $C_{p}$ (dashed line) and vortex force coefficient $C_{v}$ (solid line) and (c) FFT of the normalized vortex force coefficient $C_{v}$ at four values of the reduced velocity for a rotating parameter of $\tilde{k}_{1}=1.0$.

Fig. 2.10a shows $A^{*}$ variation with $U^{*}$ for $\tilde{k}_{1}=1.0$ as well as the vorticity field at 4 selected values of $U^{*}$. With respect to the non-rotating case $\left(\tilde{k}_{1}=0\right)$, the transition between the initial and the lower branch has been slightly advanced $\left(U^{*}=4.6\right)$ and the synchronization range finishes earlier at $U^{*} \simeq 7$. Regarding the wake pattern, the behavior is similar to that found for $\tilde{k}_{1}=0$. For high oscillations (II-III), a $\mathrm{C}(2 \mathrm{~S})$ mode is found and a $2 \mathrm{~S}$ mode for low amplitude oscillations (I-IV). Regarding $f^{*}$ and $f_{v}^{*}$ behavior, as can be seen in Fig. 2.11a for all values of $U^{*}$ both frequencies match approximately and are always synchronized when oscillations are significant. Both grow rapidly with $U^{*}$ until a value close to 1 is achieved. Due to the effect of rotation, both frequencies increase slightly over 1 until a reduced velocity of $U^{*} \simeq 7$ where the cylinder stops undergoing significant oscillations and $f^{*}$ and $f_{v}^{*}$ follow the Strouhal rule. Similar to the pure VIV curve, $C_{v}$ undergoes a $180^{\circ}$ phase change related to 
the transition between the initial and lower branch. Also in point IV, the phase of $C_{y}$ has changed by $180^{\circ}$ due to $f^{*}>1$. Also noticeably, for all values of $\tilde{k}_{1}>0, C_{y}, C_{v}$ and $C_{p}$ remain sinusoidal with only one dominant frequency in the FFT for $f_{v}^{*}$.

For negative values of $\tilde{k}_{1}$, two different values of $\tilde{k}_{1}$ are investigated in order to observe differences in wake pattern and in transverse fluid forces between VIV-type of oscillations and galloping-type of oscillations. First, the flow field around the cylinder and transverse fluid force coefficients when $\tilde{k}_{1}=-0.5$ are shown in Figs. 2.12-2.13. As can be seen, the transition between the initial to lower branch is delayed slightly until $U^{*}=5.0$ with a maximum amplitude of $A^{*}=0.64$. Furthermore, the synchronization region is notably increased. Regarding the wake pattern, a $\mathrm{C}(2 \mathrm{~S})$ mode of vortex formation is seen at $U^{*}=5(\mathrm{I})$. The picture is different for a slightly higher value of the reduced velocity (II), where an intermediate $2 \mathrm{~S}$ mode develops and slowly changes to a $\mathrm{P}$ mode in point III. At $U^{*} \simeq 10$ (point IV) there is a desynchronization between the cylinder's oscillation frequency and the vortex emission frequency, which leads to a diminishing in the amplitude of oscillation and a non-distinguishable vortex mode formation. Importantly, it seems from the flow field, that most of the range with significant oscillations occur under a vortex synchronization regime. These large amplitude oscillations also occur for $f^{*} \simeq f_{v}^{*} \simeq 1$ (Fig. 2.13a), except at point IV, where oscillations are still significant but receding and the main frequency of $f_{v}^{*}$ is associated to the Strouhal frequency. Therefore, for this $\tilde{k}_{1}$, the oscillations are mainly driven by a VIV-type of oscillation.

Fig. 2.13b shows the time evolution of fluid force coefficients $C_{y}, C_{p}, C_{v}$ at selected values of $U^{*}$ (points I to IV). In point I, $C_{y}$ and $C_{p}$ are in phase with the cylinder's displacement whereas $C_{v}$ is out of phase (undergoing a $180^{\circ}$ phase shift associated to the jump between the initial and lower branch) and for points II and III, the picture does not vary much. For point IV, $C_{v}$ has a distinctively different main frequency as seen in Fig. 2.13c showing the FFT for $C_{v}$. Points I-II-III correspond to cases under the synchronized region with the dominant frequency being $f_{v}^{*}=f^{*}$ and a small component corresponding to $3 f^{*}$. Point III also has a small component associated to the Strouhal frequency. In Point IV, synchronziation has finished and the main frequency corresponds to the Strouhal frequency though not purely sinusoidal due to terms associated to $f^{*}$.
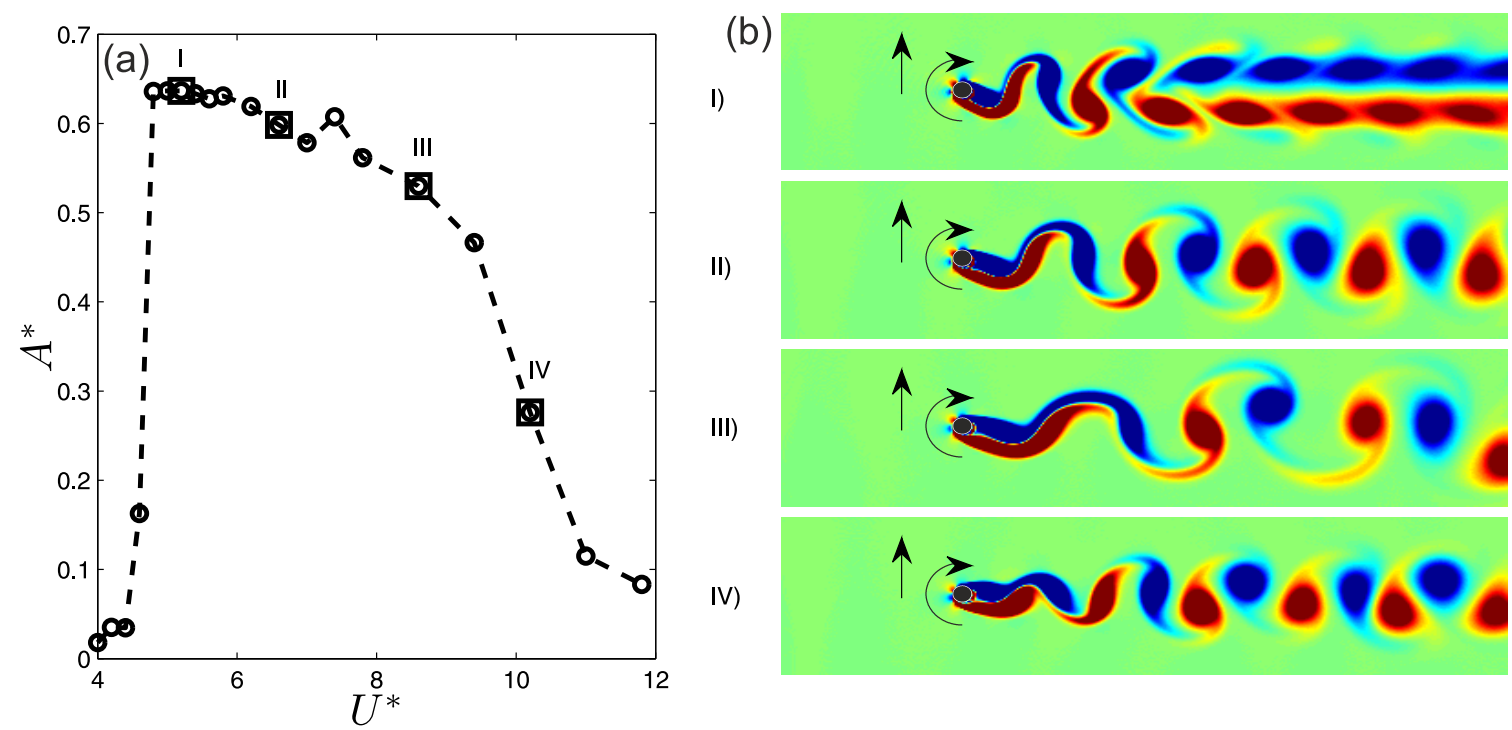

Figure 2.12: (a) Non-dimensional amplitude of oscillation and (b) vorticity field at four different values of the reduced velocity for a rotating parameter of $\tilde{k}_{1}=-0.5$.

As seen earlier (and in Fig. 2.14a) the response for $\tilde{k}_{1}=-1.2$ is markedly different from others, since really significant oscillations occur under all reduced velocities above 5 . After the initial-to- 
(a)

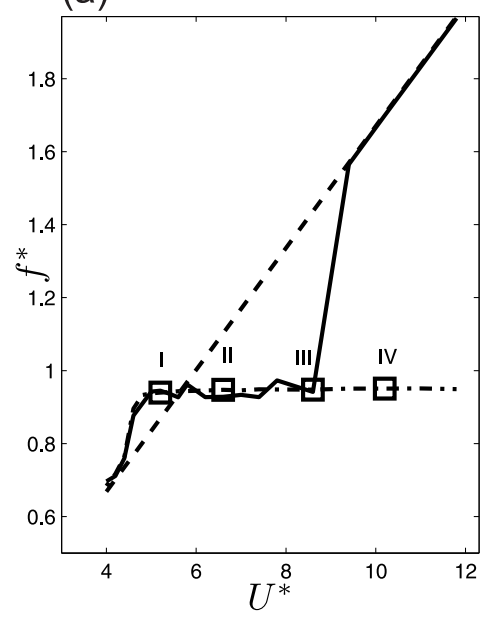

(b)

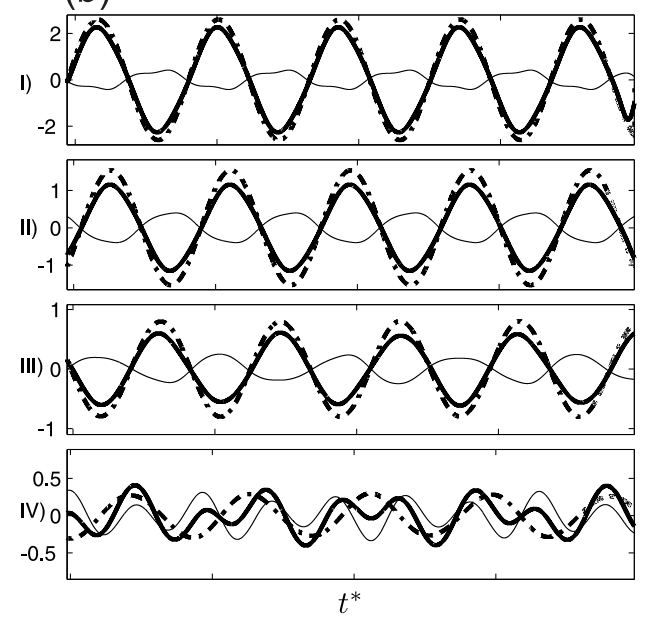

(c)

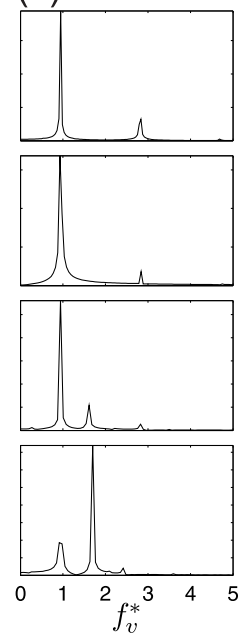

Figure 2.13: (a) Normalized frequency of oscillation $f^{*}$ (dashed line) and normalized frequency of vortex emission $f_{v}^{*}$ (solid line), (b) total transverse force coefficient $C_{y}$ (bold solid line), potential force coefficient $C_{p}$ (dashed line) and vortex force coefficient $C_{v}$ (solid line) and (c) FFT of the normalized vortex force coefficient $C_{v}$ at four values of the reduced velocity for a rotating parameter of $\tilde{k}_{1}=-0.5$.

lower branch transition (point I) $A^{*}$ shows a quasi-linear dependence with $U^{*}$ which resembles a galloping-type response. A look at the fluid forces in the cylinder helps to explain this behavior: after the initial-to-lower transition (point I), the frequency of fluid forces associated to the vortex $\left(C_{v}\right.$ coefficient) is approximately twice of the oscillation frequency (see Fig. 2.15b, point I and Figs. 2.15a and 2.15c). Up to $U^{*} \simeq 8$ (point II) the frequency of $C_{v}$ is fairly constant at twice the frequency of oscillation, and $C_{y}$ and $C_{p}$ are in phase with the cylinder's dynamics, and then $f_{v}^{*}$ grows linearly with $U^{*}$ and independently from $f^{*}$ following similarly the Strouhal frequency. Note that in point II, $f_{v}^{*}$ is clearly separated from $f^{*}$. At $U^{*} \simeq 14.4$ (point III), a drop in the amplitude of oscillation can be noted in a small range of reduced velocities where the frequency of vortex shedding $f_{v}^{*}$ is approximately three times the frequency of oscillation (see Fig. 2.15a). After that, a jump in $A^{*}$ can be observed and the linear trend with $U^{*}$ is recovered. Also, $f_{v}^{*}$ resumes a linear trend with the reduced velocity $\left(f_{v}^{*}\right.$ and $f^{*}$ grow more apart with $\left.U^{*}\right)$. Regarding fluid forces coefficients, separation between $C_{v}, C_{y}$ and $C_{p}$ is progressively more clear with very different timescales. In point $\mathrm{I}$, the flow pattern corresponds to that of a $\mathrm{C}(2 \mathrm{~S})$ mode (Fig. 2.14b). In II, a $\mathrm{P}+\mathrm{S}$ mode is observed which is responsible for the fluid forces associated to the vortex term $\left(C_{v}\right)$ to have $f_{v}^{*} \simeq 2 f^{*}$. In point III, which corresponds to $U^{*}=14.4$ (approximately three times the Strouhal number of the cylinder at rest), the flow field is characterized by a vortex shedding mode with three vortices shed per half cycle, which corresponds to a $2 \mathrm{~T}$ mode (triplets of vortices shed per side of the oscillation period as described in Bourguet and Jacono, 2014). Finally in IV, $f_{v}^{*}$ and $f^{*}$ are very different and thus, the vortex formation is decoupled from the oscillation of the cylinder. The vortices are shed independently from the cylinder's oscillation at their own frequency and thus the wake pattern resembles that of a wave as each vortex is shed at different points of the cycle of oscillation. This indicates that for $U^{*}>15$ and $\tilde{k}_{1}<-1$, where $f_{v}^{*}>3 f^{*}$, oscillations are not caused by resonance with the vortices shed but by a galloping-type of mechanism where amplitude grows almost linearly with $U^{*} . f^{*}$ is almost constant (for large enough reduced velocity) and thus the characteristic time of vortex shedding and the characteristic time of oscillation are clearly separated for high enough $U^{*}$. In Section 2.4, a quasi-steady model is presented (where the vortex terms are neglected), to predict the amplitude of oscillation as well as the limiting value for $\tilde{k}_{1}$ where the response of the circular cylinder changes from VIV-type of solutions to a galloping-type of solution. 


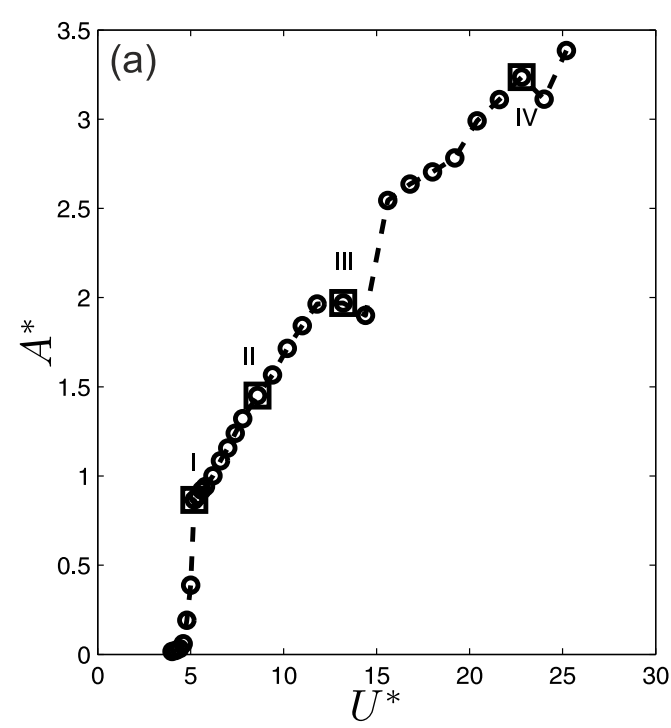

(b)

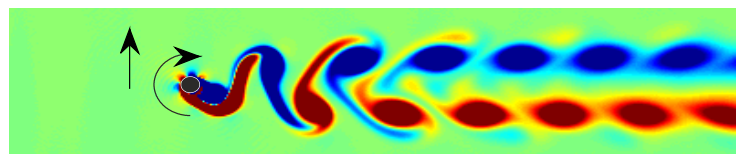

II)

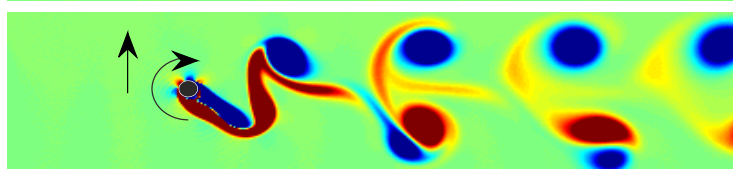

III)

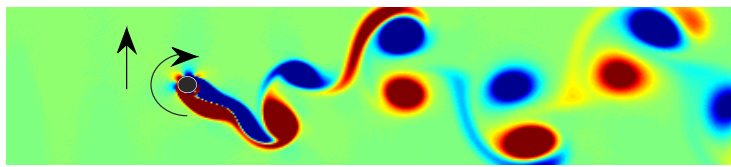

IV)

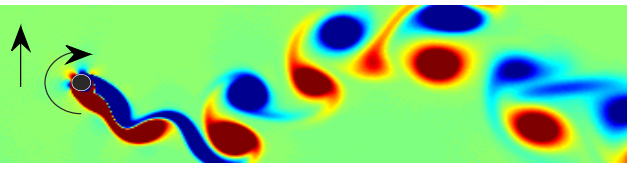

Figure 2.14: (a) Non-dimensional amplitude of oscillation and (b) vorticity field at four different values of the reduced velocity for a rotating parameter of $\tilde{k}_{1}=-1.2$.

(a)

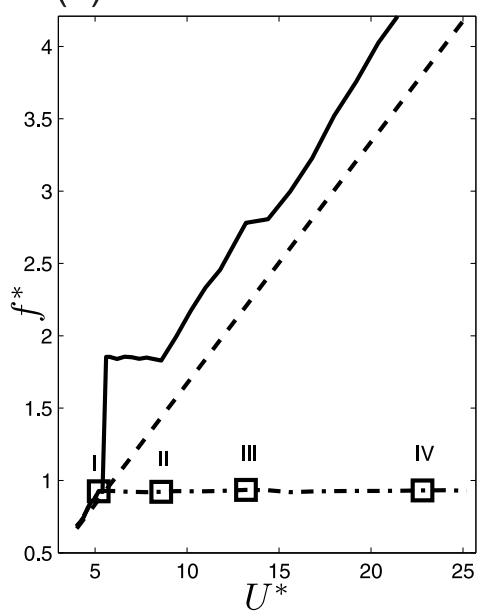

(b)
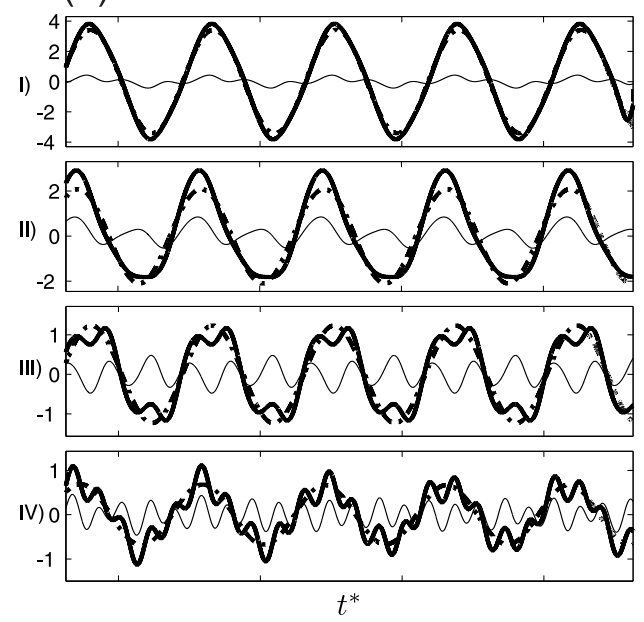

(c)

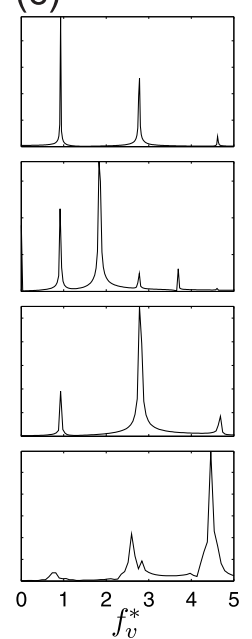

Figure 2.15: (a) Normalized frequency of oscillation $f^{*}$ (dashed line) and normalized frequency of vortex emission $f_{v}^{*}$ (solid line), (b) total transverse force coefficient $C_{y}$ (bold solid line), potential force coefficient $C_{p}$ (dashed line) and vortex force coefficient $C_{v}$ (solid line) and (c) FFT of the normalized vortex force coefficient $C_{v}$ at four values of the reduced velocity for a rotating parameter of $\tilde{k}_{1}=-1.2$.

\subsubsection{Rotation proportional to the cylinder's velocity $\left(\theta=\tilde{k}_{2} Y^{\prime}\right)$}

Another strategy is proposed to modify the response of a circular cylinder undergoing VIV by rotating the cylinder proportionally to its own velocity in order to be able to either enhance or reduce VIV effects and to modify the flow field and the wake pattern behind the circular cylinder. Results for rotating the cylinder proportional to the velocity of oscillation are here presented.

$$
\theta=\tilde{k}_{2} Y^{\prime}
$$

where $\tilde{k}_{2}$ is a proportional constant. 


\subsubsection{Cylinder dynamics}
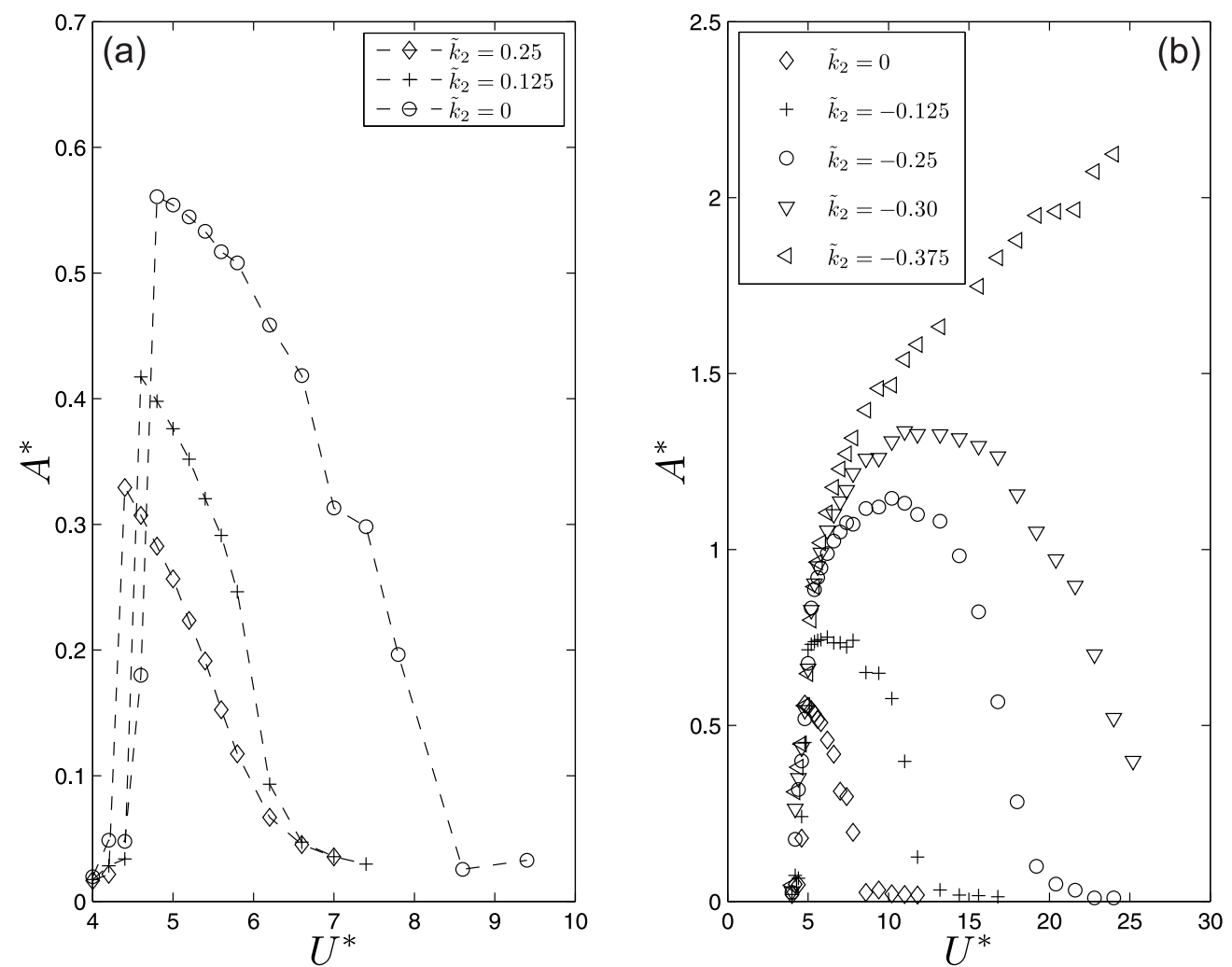

Figure 2.16: (a) Maximum non-dimensional amplitude oscillation as a function of the reduced velocity for positive values of the rotating parameter $\tilde{k}_{2}$ and (b) for negative values of $\tilde{k}_{2}$.

In Fig. 2.16, the amplitude of oscillation of the cylinder is plotted as a function of the reduced velocity for different values of $\tilde{k}_{2}$. For $\tilde{k}_{2}>0$ VIV response is attenuated, with reduced values of $A^{*}$, and the transition from initial-to-lower branch is anticipated. Also, the synchronization region that undergoes VIV-type of oscillations is reduced. This behavior is more pronounced as $\tilde{k}_{2}$ is larger. For negative values of $\tilde{k}_{2}$, the range undergoing large amplitude oscillations is increased as well as the maximum amplitude obtained. Interestingly, the transition between the initial and lower branch is not clear. For different levels of negative actuation, there is a range of reduced velocity where all of the amplitude response's curves collapse into a straight. As $\tilde{k}_{2}$ becomes more negative, the curve keeps for a longer range of reduced velocity at this limiting straight line until the slope of the response curve changes departing from this line. Similarly to the previous strategy (proportional to cylinder's position, $\tilde{k}_{1}$ ), the maximum amplitude can be enlarged or decreased importantly, though the necessary actuation to do so is bigger. All curves obtained for $\tilde{k}_{2}$ exhibit well defined VIV-type characteristics, even for large negative values of $\tilde{k}_{2}$.

In Fig. 2.17 the non-dimensional frequency of oscillation $\left(f^{*}\right)$ is plotted as a function of the reduced velocity for different values of $\tilde{k}_{2}$. As a general trend for $\tilde{k}_{2}>0, f^{*}$ is lowered importantly from its natural frequency for low reduced velocities, but it increases rapidly with the reduced velocity until the initial-to-lower branch transition, afterwards, $f^{*}$ increases slightly with the reduced velocity until desynchronization happens. Such desynchronization happens for lower values of $U^{*}$ as $\tilde{k}_{2}$ increases. On the other hand, for negative values of $\tilde{k}_{2}$, after the initial-to-lower branch, the frequency of oscillation increases with the reduced velocity with increasing slope as $\tilde{k}_{2}$ is more negative until desynchronization 

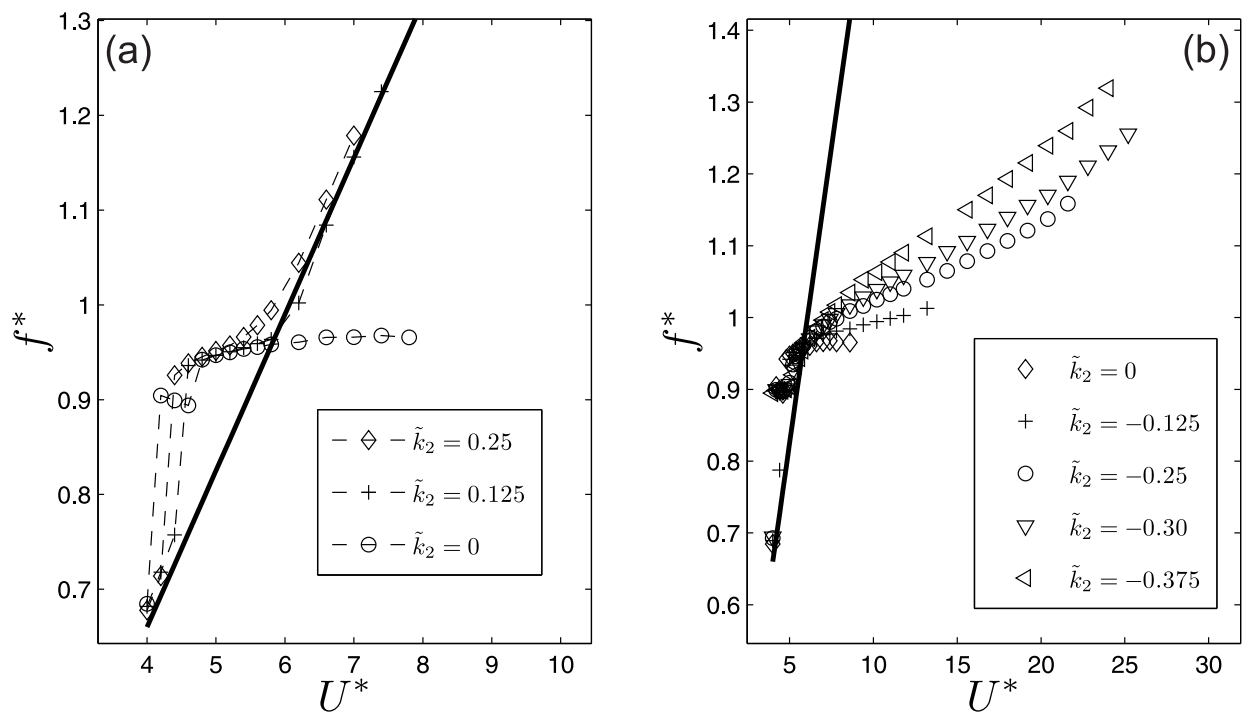

Figure 2.17: (a) Oscillating frequency of the cylinder as a function of the reduced velocity for positive values of the rotating parameter $\tilde{k}_{2}$ and (b) for negative values of $\tilde{k}_{2}$. Strouhal law is shown in bold line.

occurs.

\subsubsection{Flow field and fluid forces}

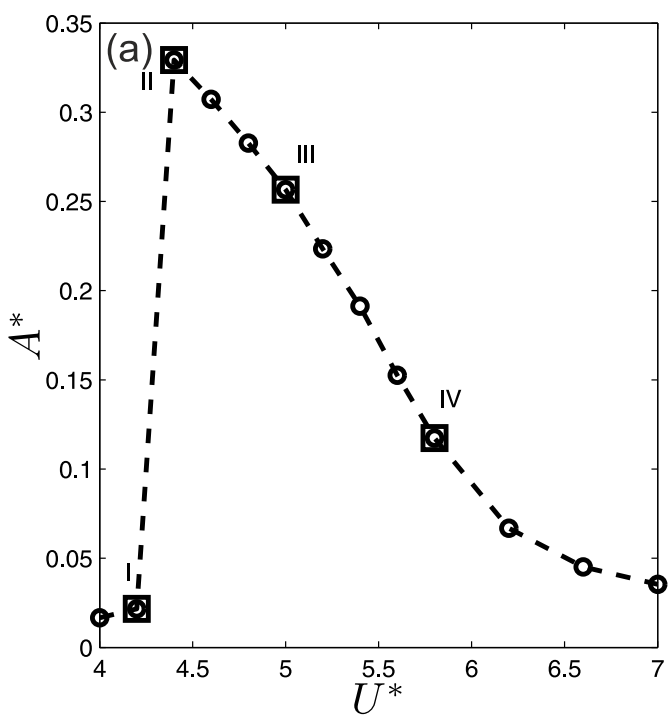

(b)

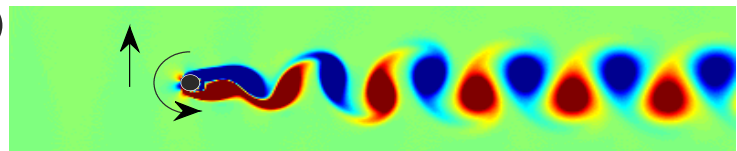

II)

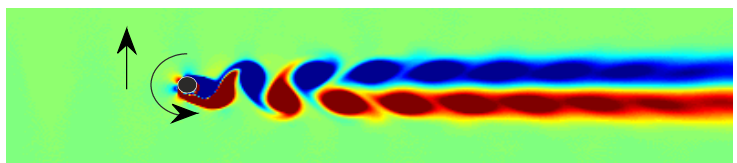

III)
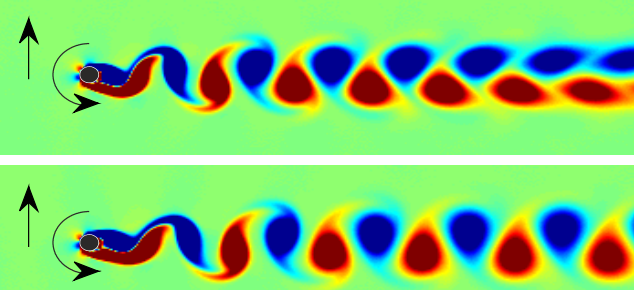

Figure 2.18: (a) Non-dimensional amplitude of oscillation and (b) vorticity field at four different values of the reduced velocity for a rotating parameter of $\tilde{k}_{2}=0.25$.

In Fig. 2.18, the amplitude response for $\tilde{k}_{2}=0.25$ is given as well as the flow field at given reduced velocities. With respect to the wake pattern, these are similar to the ones obtained for the classical VIV without actuation. For high oscillations, a C(2S) (II) mode of shedding vortex is obtained and for lower oscillations it is equivalent to a $2 \mathrm{~S}$ vortex shedding mode (I-II-IV).

Fig. 2.19a compares $f^{*}$ and $f_{v}^{*}$. For all values of the reduced velocity both frequencies match approximately, this is, the frequency of oscillation and the frequency of vortex emission are synchro- 
(a)

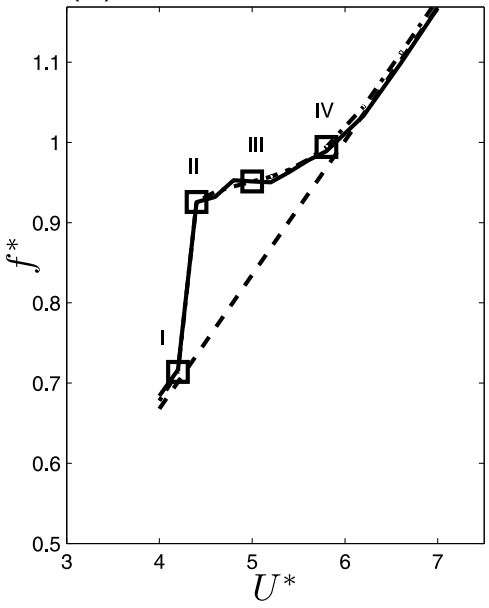

(b)

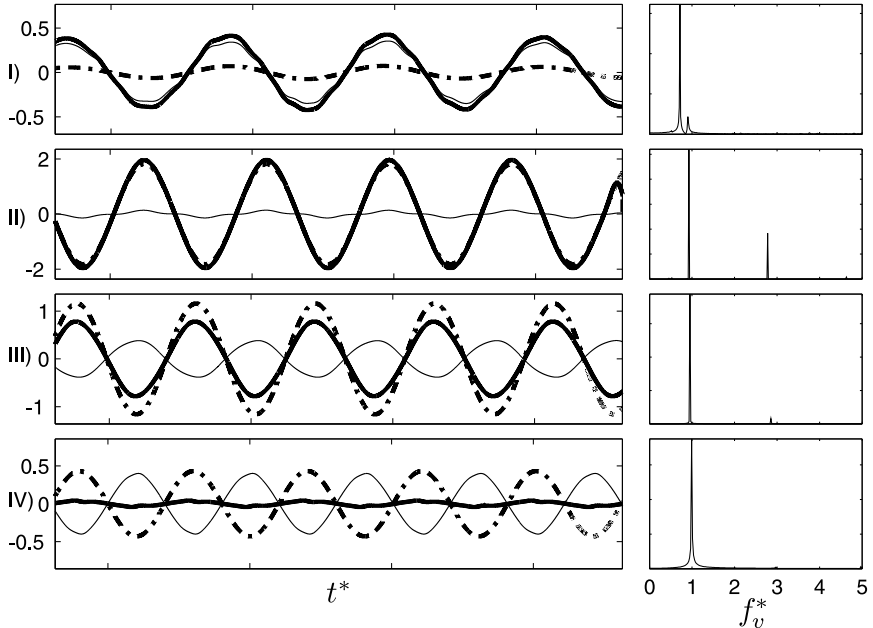

Figure 2.19: (a) Normalized frequency of oscillation $f^{*}$ (dashed line) and normalized frequency of vortex emission $f_{v}^{*}$ (solid line), (b) total transverse force coefficient $C_{y}$ (bold solid line), potential force coefficient $C_{p}$ (dashed line) and vortex force coefficient $C_{v}$ (solid line) and (c) FFT of the normalized vortex force coefficient $C_{v}$ at four values of the reduced velocity for a rotating parameter of $\tilde{k}_{2}=0.25$.

nized. Both frequencies increase rapidly until a value close to 1 , and due to the effect of the rotation, the frequency increases with the reduced velocity until a reduced velocity of $U^{*} \approx 6$ (point IV) where the frequency of oscillation and of the vortex force begin to follow the Strouhal rule. When the jump between the initial and lower branch occurs, $C_{v}$ is out of phase with respect to $C_{p}$ and $C_{y}$ at $U^{*}=4.5$ (II). At $U^{*} \simeq 5.7$ (point IV), the total force is approximately 0 , the frequency of oscillation is $f^{*}=1$, from here the sign of $C_{y}$ changes, thus being out of phase with the cylinder's displacement.

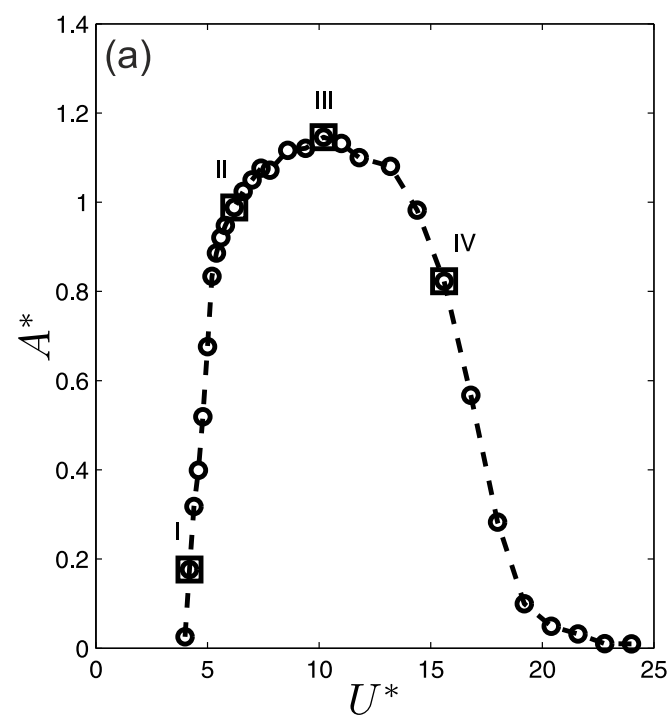

(b)

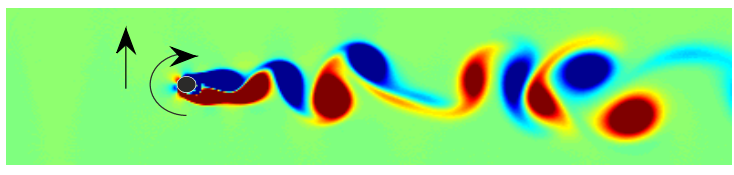

II)

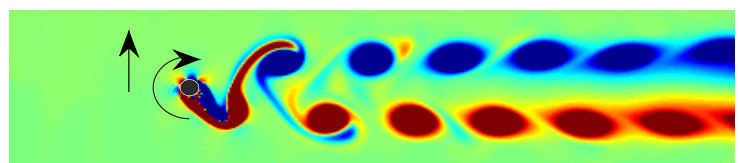

III)

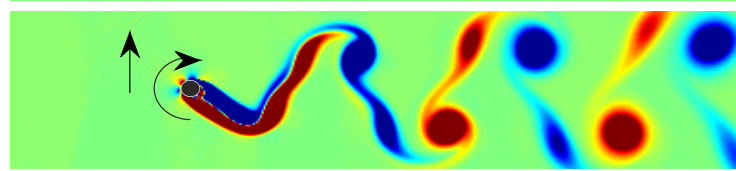

IV)

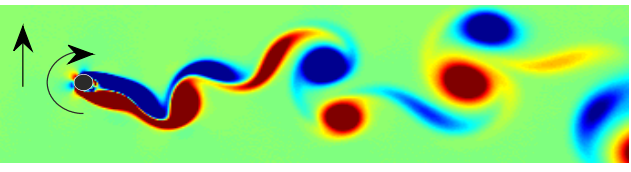

Figure 2.20: (a) Non-dimensional amplitude of oscillation and (b) vorticity field at four different values of the reduced velocity for a rotating parameter of $\tilde{k}_{2}=-0.25$.

Two negative values of $\tilde{k}_{2}$ are investigated. In first place, in Figs. 2.20-2.21, the flow field and the fluid forces of a cylinder undergoing oscillations for rotation parameter $\tilde{k}_{2}=-0.25$ are presented. As it can be seen, there is no proper transition between the initial and the lower branch. In this case, the 
(a)

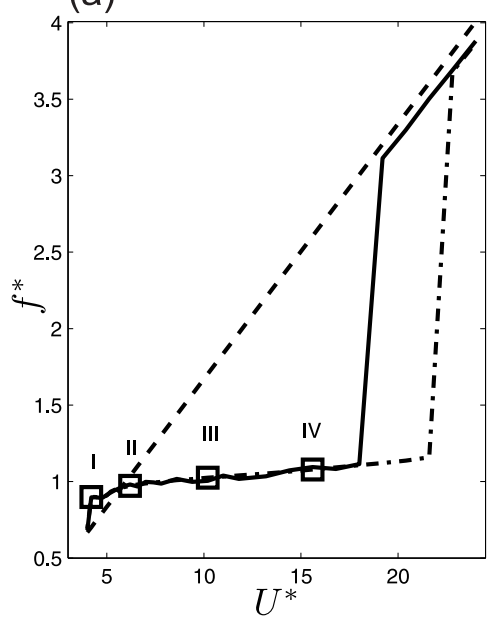

(b)

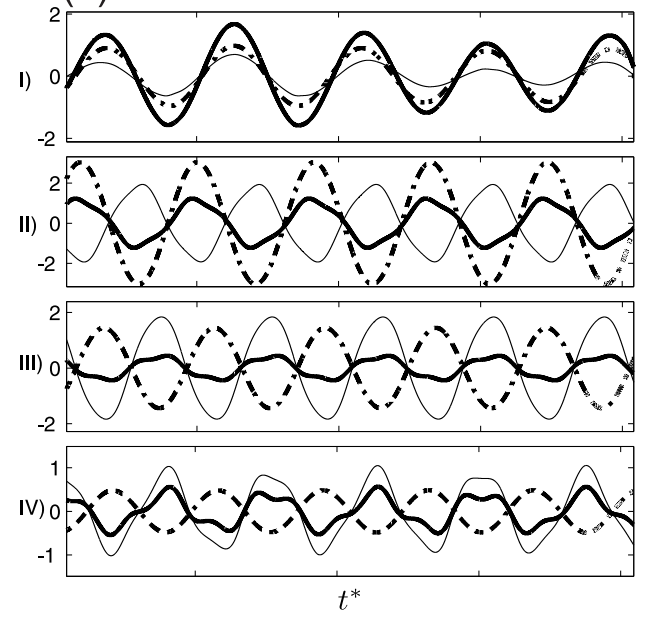

(c)

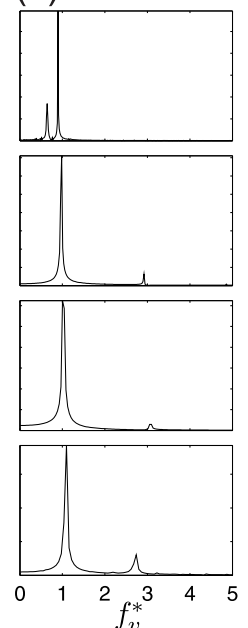

Figure 2.21: (a) Normalized frequency of oscillation $f^{*}$ (dashed line) and normalized frequency of vortex emission $f_{v}^{*}$ (solid line), (b) total transverse force coefficient $C_{y}$ (bold solid line), potential force coefficient $C_{p}$ (dashed line) and vortex force coefficient $C_{v}$ (solid line) and (c) FFT of the normalized vortex force coefficient $C_{v}$ at four values of the reduced velocity for a rotating parameter of $\tilde{k}_{2}=-0.25$.

amplitude's oscillations depart from the limiting straight at $U^{*}=5$ and further continues increasing with diminishing slope until a maximum amplitude of $A^{*}=1.18$ at a reduced velocity of $U^{*}=10.2$. Furthermore, the region of large oscillations (synchronization) is increased importantly. With respect to the flow field of vortex formation, point I corresponds to the beginning of the transition between the initial and lower branch. In this case, there is no clear vortex shedding wake which is a common characteristic for all negative values of $\tilde{k}_{2}$ in this region of transition. In II, a $\mathrm{C}(2 \mathrm{~S})$ mode develops after the transition between the initial and lower branches occurs. Slowly it changes to a $2 \mathrm{P}$ vortex mode in III. This mode occurs over a wide range of reduced velocity. In IV the vortex mode has started to desynchronize and thus the vortex shedding structure is not so clear. With respect to the forces exerted on the cylinder, in Fig. 2.21b the force coefficients are given. For negative values of $\tilde{k}_{2}$ the forces are always synchronized with the oscillation frequency for large amplitude oscillations, $f_{v}^{*}=f^{*}$ (Fig. 2.21a). In point II, $C_{v}$ has undergone a $180^{\circ}$ phase shift associated to the lower branch of VIV and in III $C_{y}$ also suffers such phase shift and thus, $f^{*}>1$.

Figs. 2.22- 2.23 correspond to the flow field and fluid forces coefficients for the case $\tilde{k}_{2}=-0.375$. Regarding the amplitude of oscillation, there is no proper transition between the initial and the lower branch. In this case, the amplitude's oscillations (Fig. 2.22a) depart from the limiting straight at $U^{*} \simeq 7$ and further continues increasing with diminishing slope. Furthermore, the region of large oscillations is increased very importantly. With respect to the flow field of vortex formation, I and II, corresponds to the beginning of the transition between the initial and lower branch. In this case, there is no clear vortex shedding pattern. In point II, a $\mathrm{C}(2 \mathrm{~S})$ can be seen but as it is still in the transition region the results are dirtied. Slowly it changes to a $2 \mathrm{P}$ vortex mode in III which is different from the $2 \mathrm{P}$ mode observed for $\tilde{k}_{2}=-0.25$ (Fig. 2.20b). The maximum acceleration of the rotation occurs for $Y=0$ and it induces the primary vortex to be shed at the centerline. In this sense, it can be defined as a $2 \mathrm{~S}$ mode with a secondary vortex shed in the exterior. This mode occurs over a wide range of reduced velocity, developing in point IV also as a $2 \mathrm{~S}$ mode with a secondary vortex which has been further developed from point III. With respect to the forces exerted on the cylinder, in Fig. 2.23b the force coefficients $\left(C_{y}, C_{p}, C_{v}\right)$ and in Fig. 2.23c the FFT of $C_{v}$ are given. As for $\tilde{k}_{2}=-0.25$ the forces are always synchronized with the oscillation frequency $\left(f_{v}^{*}=f^{*}\right)$. Similar considerations about the 


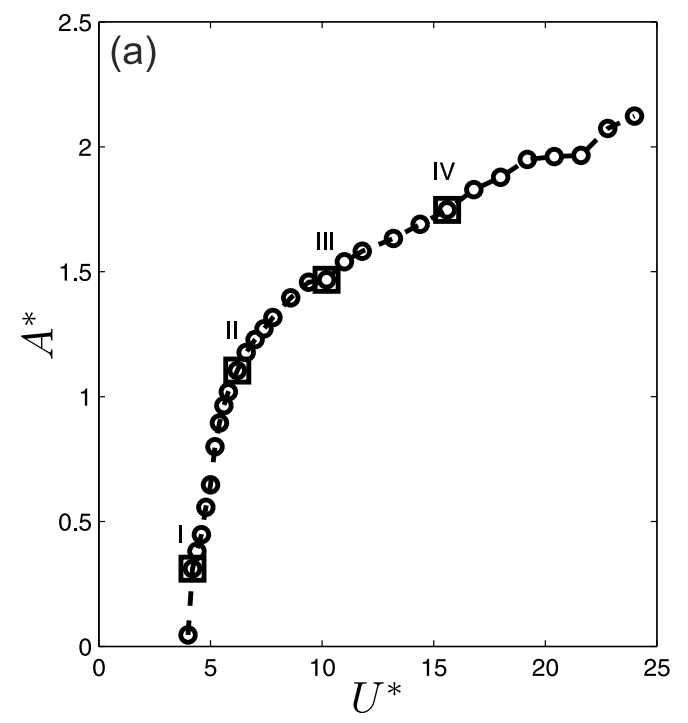

(b)

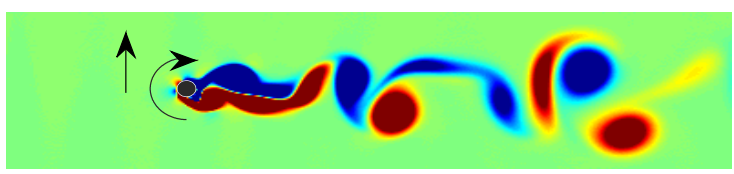

II)
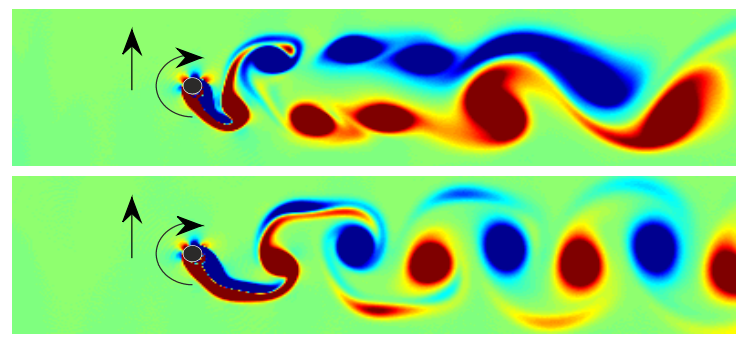

IV)

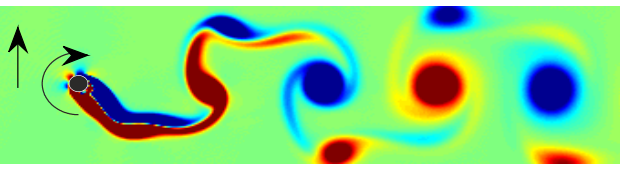

Figure 2.22: (a) Non-dimensional amplitude of oscillation and (b) vorticity field at four different values of the reduced velocity for a rotating parameter of $\tilde{k}_{2}=-0.375$.

(a)

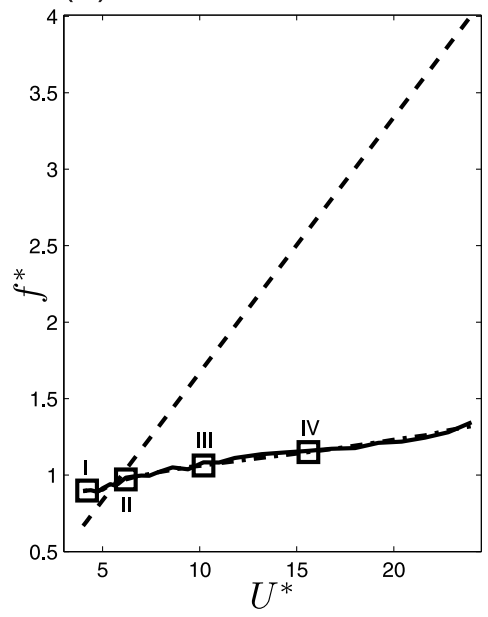

(b)

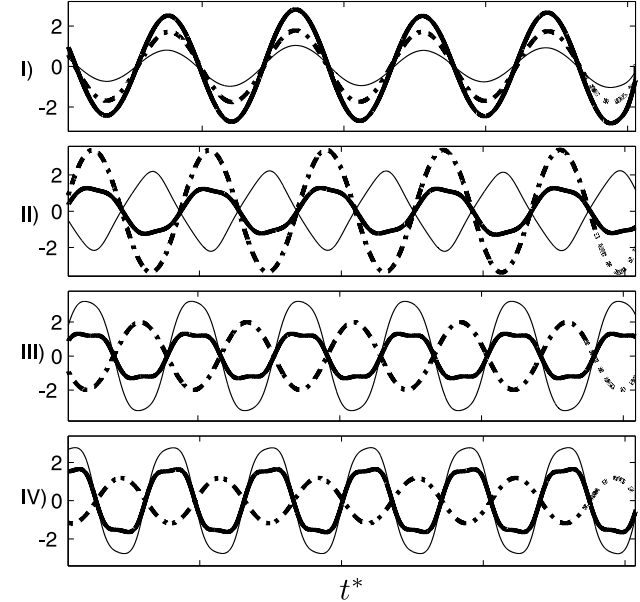

(c)

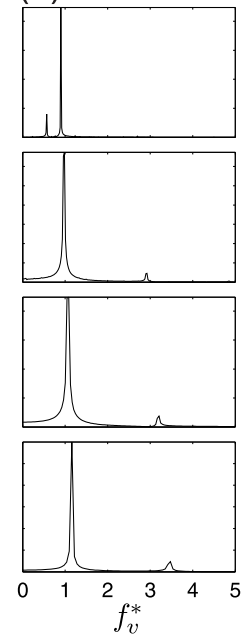

Figure 2.23: (a) Normalized frequency of oscillation $f^{*}$ (dashed line) and normalized frequency of vortex emission $f_{v}^{*}$ (solid line), (b) total transverse force coefficient $C_{y}$ (bold solid line), potential force coefficient $C_{p}$ (dashed line) and vortex force coefficient $C_{v}$ (solid line) and (c) FFT of the normalized vortex force coefficient $C_{v}$ at four values of the reduced velocity for a rotating parameter of $\tilde{k}_{2}=-0.375$.

phase changes of the different force coefficients are obtained to those of $\tilde{k}_{2}=-0.25$.

Differently from the previous law (rotation proportional to the cylinder's displacement), all of $\tilde{k}_{2}$ investigated have VIV-type of response. When the cylinder undergoes significant oscillations, vortex emission, fluid forces and the cylinder's oscillation all have the same frequency. The region of synchronization may be greatly increased, however, oscillations occur under similar pattern and as will be investigated in the following section, no possible quasi-steady solution may be obtained. 


\subsection{Quasi-steady analysis}

As observed in the previous section, for $\theta=\tilde{k}_{1} Y$, there is a clear separation in the dynamical behavior between the cases for $\tilde{k}_{1} \geq-1$ and for those where $\tilde{k}_{1}<-1$. Summarizing, for $\tilde{k}_{1}>-1$, the dynamics of the cylinder is similar to a VIV-type of phenomenon where the frequency of oscillation is in synchronization with the frequency of vortex emission and thus the cylinder undergoes large oscillations. However, as the reduced velocity increases, the synchronization stops, and large amplitude oscillations disappear. $\tilde{k}_{1}$ only modifies the amplitude of oscillation and enhances or diminishes the region of synchronization but it does not modify the dynamical behavior of the cylinder. On the other hand, for large enough negative values of $\tilde{k}_{1}\left(\tilde{k}_{1}<-1\right)$, the amplitude of oscillation seems to increase linearly with the reduced velocity without limit, leading to assume some kind of motion-induced vibration (or galloping) phenomenon is happening. The frequency of vortex emission increases linearly with the reduced velocity (following closely the Strouhal law) independently from the frequency of oscillation of the cylinder which remains fairly constant, thus, the characteristic time of vortex emission and the characteristic time of oscillations are importantly separated (for high enough values of the reduced velocity $U^{*}>15$ ). These aspects of the response of the cylinder's dynamics lead us to believe that there is a quasi-steady solution for high enough reduced velocities, similarly to the galloping phenomenon, where it can be studied with the quasi-steady hypothesis when the characteristic time of vortex emission is much smaller than the characteristic time of oscillation.

For the quasi-steady analysis (QS), it is supposed that the transverse force at each instant can be obtained from the steady lift and drag force with an equivalent velocity with an angle of attack. In Fig. 2.24a a scheme of an equivalent configuration is given.

(a)

(b)
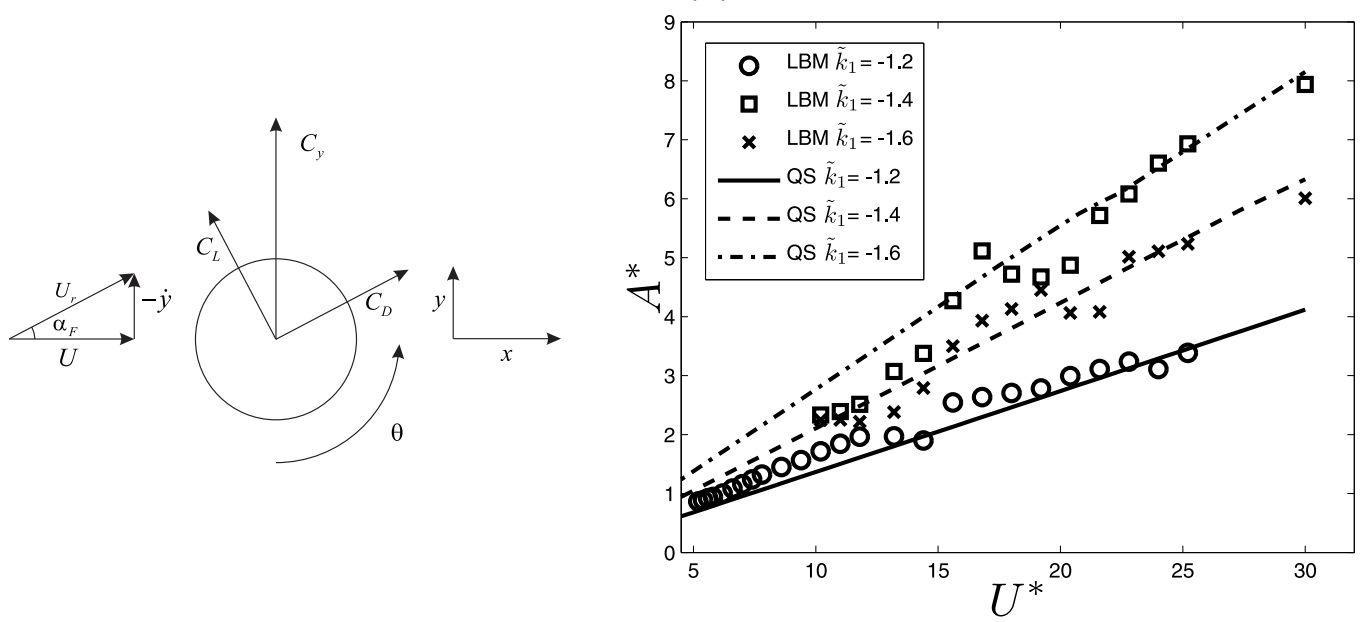

Figure 2.24: (a) Schematics of the forces acting on the cylinder in a quasi-steady situation. (b) Comparison between quasi-steady solutions and LBM numerical analysis for different values of $\tilde{k}_{1}$.

After projecting the lift and the drag forces, the transverse force coefficient can be obtained as:

$$
C_{y}=\frac{U_{r}}{U}\left(-C_{L} \cos \left(\alpha_{F}\right)-C_{D} \sin \left(\alpha_{F}\right)\right)=-\frac{C_{L}}{\cos \left(\alpha_{F}\right)}-C_{D} \frac{\tan \left(\alpha_{F}\right)}{\cos \left(\alpha_{F}\right)}
$$

where $\alpha_{F}$ is the instantaneous angle of attack, $\tan \left(\alpha_{F}\right)=-\dot{y} / U, \cos \left(\alpha_{F}\right)=U / U_{r}, U_{r}$ is the relative incident flow speed $\left(U_{r}=\sqrt{U+\dot{y}^{2}}\right)$ and $C_{L}$ and $C_{D}$ are, respectively, the lift and drag fluid force coefficients. $C_{L}$ and $C_{D}$ can be obtained from the 'static' configuration as a function of the rotation rate. Fig. 2.5 shows this dependence from numerical simulations carried via LBM in the present study. Let us assume for the present study a linear variation with the rotation rate for both 
$C_{L}$ and $C_{D}$ :

$$
\begin{gathered}
C_{L}=C_{L \alpha} \frac{\dot{\theta} R}{U_{r}} \\
C_{D}=C_{D o}-C_{D \alpha}\left|\frac{\dot{\theta} R}{U_{r}}\right| .
\end{gathered}
$$

When the cylinder rotates proportionally to the cylinder's displacement $\left(\dot{\theta}=k_{1} \dot{y}\right)$, one has

$$
\begin{gathered}
C_{L}=\frac{C_{L \alpha}}{2} \tilde{k}_{1} \frac{\dot{y}}{U_{r}}, \\
C_{D}=C_{D o}-\frac{C_{D \alpha}}{2}\left|\tilde{k}_{1} \frac{\dot{y}}{U_{r}}\right| .
\end{gathered}
$$

Introducing these force coefficients $C_{L}$ and $C_{D}$ into Eq. 2.20 and since $\dot{y} / U_{r}=Y^{\prime} / U^{*} \cos \left(\alpha_{F}\right)$ it follows

$$
C_{y}=-\frac{C_{L \alpha}}{2} \tilde{k}_{1} \frac{Y^{\prime}}{U^{*}}-C_{D o} \frac{Y^{\prime}}{U^{*}} \sqrt{1+\left(\frac{Y^{\prime}}{U^{*}}\right)}+\frac{C_{D \alpha}}{2}\left|\tilde{k}_{1} \frac{Y^{\prime}}{U^{*}}\right| \frac{Y^{\prime}}{U^{*}} .
$$

The first term in Eq. 2.23 can be viewed as a linear damping $\left(\tilde{k}_{1}>0\right)$ or a linear negative damping term $\left(\tilde{k}_{1}<0\right)$. The last two terms are non-linear damping terms associated to the drag force. Note there are no stiffness terms that could change significantly the frequency of oscillation. An expression around $Y^{\prime} / U^{*}$ could be made to obtain the amplitude of oscillations analytically as in Naudascher and Rockwell (2004) or Barrero-Gil et al. (2009, 2010). However, it is not the scope of this study to develop an analytical solution of the problem so the dynamical equation for the cylinder (Eq. 2.3a) is solved with a Runge-Kutta scheme with $C_{y}$ given by Eq. 2.23.

The $A^{*}$ variation with $U^{*}$ given by the LBM simulation is compared to the QS analysis in Fig. 2.24b for $\tilde{k}_{1}=-1.2,-1.4,-1.6$. It can be seen that the QS prediction works reasonably well and the errors are bounded to lower than $10 \%$, which seems to be accurate enough to predict the cylinder's amplitude of oscillation for high $U^{*}$.

It is of interest to study the value of $\tilde{k}_{1}$ from which the galloping-type response is expected to occur accordingly to the QS model. Eq. 2.23 can be expanded in Taylor series around $Y^{\prime} / U^{*}$. If an expansion up to third order is chosen,

$$
C_{y}=-\frac{C_{L \alpha}}{2} \tilde{k}_{1} \frac{Y^{\prime}}{U^{*}}-C_{D o}\left(\frac{Y^{\prime}}{U^{*}}+\frac{1}{2}\left(\frac{Y^{\prime}}{U^{*}}\right)^{3}\right)+\frac{C_{D \alpha}}{2}\left|\tilde{k}_{1} \frac{Y^{\prime}}{U^{*}}\right| \frac{Y^{\prime}}{U^{*}} .
$$

According to the QS model, galloping-type oscillations will occur when the linearized term of the transverse force coefficients yields a negative value, thus

$$
\tilde{k}_{1}<-2 \frac{C_{D o}}{C_{L \alpha}} \simeq-1.045,
$$

which agrees well with the results found during LBM computations, since galloping-type oscillations appeared for $\tilde{k}_{1}<-1$.

Now, let us make a similar analysis for the case where the cylinder is forced to rotate proportionally to the velocity of the cylinder (that is $\theta=\tilde{k}_{2} Y^{\prime}$ ) Then,

$$
C_{y}=-\frac{C_{L \alpha}}{2} \tilde{k}_{2} \frac{Y^{\prime \prime}}{U^{*}}-C_{D o} \frac{Y^{\prime}}{U^{*}} \sqrt{1+\left(\frac{Y^{\prime}}{U^{*}}\right)}+\frac{C_{D \alpha}}{2}\left|\tilde{k}_{2} \frac{Y^{\prime \prime}}{U^{*}}\right| \frac{Y^{\prime}}{U^{*}}
$$

The first term is an inertia term, which will modify the frequency of oscillation; the second and 
third terms have non-linear damping character terms. So, in this case, there is no negative damping term, and therefore, self-excited vibrations (galloping-type) are not expected to occur (no gallopingtype oscillations were observed in LBM computations) for this type of rotation mechanism. Even though, large amplitude oscillations can be obtained for large values of $U^{*}$ but these always occur under VIV conditions; vortex emission frequency, the frequency of oscillation and the fluid forces are always synchronized, thus it is not possible to neglect the effect of vortex emission making a quasi-steady analysis not possible.

\subsection{Discussion and comparison between the two rotating laws}

\subsubsection{Maximum angle of rotation}

From the previous sections, as has been studied, both rotating laws can lead to a reduction/increase of the maximum amplitude of oscillation as well as the region of reduced velocities that undergoes large amplitude oscillation. However, it is interesting to compare which rotation law requires less actuation to modify the amplitude's response. To do so, a change of variables is convenient. If a sinusoidal solution can be assumed $\left(Y=A^{*} \sin \left(2 \pi f^{*} t^{*}\right)\right)$, then the maximum angle of rotation can be obtained as

$$
\theta_{\max }=k_{1}^{*} A^{*} \quad \text { or } \quad \theta_{\max }=k_{2}^{*} A^{*}
$$

where $k_{1}^{*}=\tilde{k}_{1}$ and $k_{2}^{*}=2 \pi \tilde{k}_{2} f^{*}$. In Fig. 2.25, the contour maps for $\theta_{\max }$ are given as a function of $U^{*}$ and $k_{1}^{*}\left(k_{2}^{*}\right)$ for negative values of $k_{1}^{*}$ and $k_{2}^{*}$. As can be seen, rotating the cylinder proportionally to its displacement, higher values of $\theta_{\max }$ are obtained for lower absolute value of $k_{1}^{*}$ compared to when the cylinder is rotated proportionally to its velocity. Thus, as seen from Eq. 2.27, the same amplitude of oscillation can be obtained with less rotation when the cylinder is rotated proportionally to $k_{1}^{*}$ than with $k_{2}^{*}$, as well as the region of $U^{*}$ undergoing large amplitude of oscillations is greatly increased with less necessary rotation of the cylinder. For example, in Figs. 2.25a and 2.25b, the point highlighted corresponds to an amplitude of oscillation of $A^{*}=1.6$ at a reduced velocity of $U^{*}=15$. Whereas for the first case, such point occurs for a value of $k_{1}^{*}=-1.04$ and $\theta_{\max }=1.67$, rotation proportional to the cylinder's velocity yields a value a $k_{2}^{*}=-2.5$ and $\theta_{\max }=4$. Thus, to obtain the same level of $A^{*}$ when rotating the cylinder proportionally to its velocity at the given point of reference requires 2.4 times more actuation than when rotating the cylinder proportionally to its position. This result is also valid for positive values of $k_{1}^{*}$ and $k_{2}^{*}$, where less rotation is required for the first rotation law to diminish the amplitude of oscillation (as well as the synchronization region) when rotating it proportionally to its displacement.

\subsubsection{On the intensity of the vortices shed}

One of the questions introduced earlier was whether if the oscillations were driven under a VIV-type of oscillation or more likely under a galloping-type of oscillation. Through the amplitude of oscillation $A^{*}$, frequency of oscillation $f^{*}$, the frequency of vortex shedding $f_{v}^{*}$ as well as the wake pattern after the cylinder, it was observed that for large negative values of $\tilde{k}_{1}$ the mechanism of oscillation was modified into a galloping-type of oscillation and thus a quasi-steady model was proposed which agrees reasonably well with the LBM numerical simulations for values of $\tilde{k}_{1}<-1$. Here the intensity of the vortices shed are studied in order to determine how the different rotation laws proposed affect the intensity of the vortices shed. The non-dimensional circulation strength of the vortices shed has been computed as

$$
\Gamma^{*}=\frac{\int \nabla \times \mathbf{v d} x}{U D}
$$



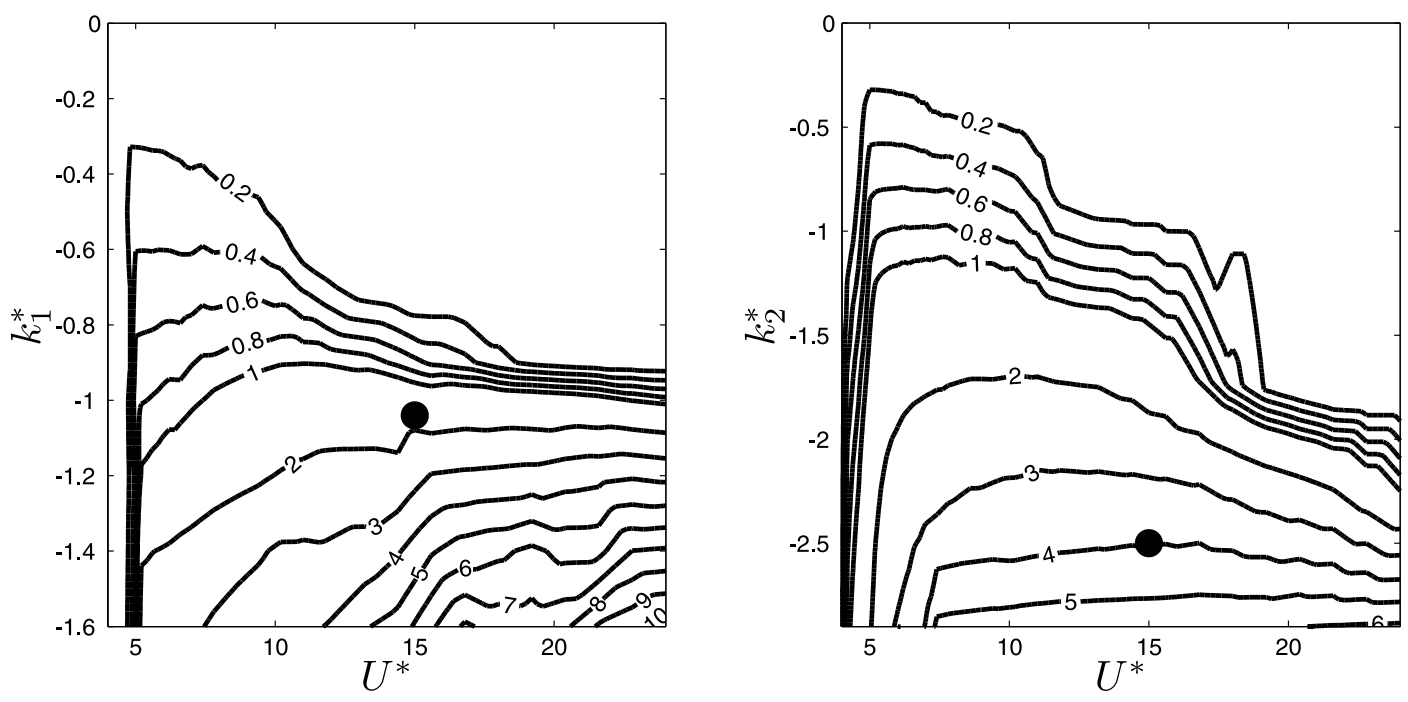

Figure 2.25: (a) Maximum angle for rotation as a function of $U^{*}$ and $k_{1}^{*}$. (b) Maximum angle for rotation as a function of $U^{*}$ and $k_{2}^{*}$.

The circulation strength is computed right when the vortex is shed and detached from the cylinder's surface. In Fig. 2.26, the circulation of the largest vortex shed at each cycle is plotted as a function of the reduced velocity for the VIV curve (used as basis), and for $\tilde{k}_{1}=-1.2$ (which suffers gallopingtype of oscillations for large enough $U^{*}$ ) and $\tilde{k}_{2}=-0.375$ (VIV-type of response) which have similar amplitude of oscillations. For the VIV curve, in the initial branch, the non-dimensional circulation is similar to the static case (cylinder at rest), after the initial-to-lower transition, the non-dimensional circulation increases up to $\Gamma^{*}=4.5$ and remains fairly constant until desynchronization occurs leading to values of the circulation close to the static case. For $\tilde{k}_{2}=-0.375$, the circulation of the vortices shed grow with the reduced velocity during the transition between the initial and lower branches until a value of $\Gamma^{*}=6.7$ and then it keeps quite constant up to large values of $U^{*}$ (as long as synchronization between oscillation and vortex emission occurs). On the other hand, for $\tilde{k}_{1}=-1.2$, the circulation grows importantly up to $\Gamma^{*}=6.3$ and then diminishes with $U^{*}$ until values of $\Gamma^{*}$ close to the static case are obtained again. This phenomenon (alongside with the characteristics of $f^{*}$ as well as $f_{v}^{*}$ ) highlights the fact that for large enough values of $\tilde{k}_{1}$, the oscillations are not driven by a resonance with the vortex emission but by the quasi-steady value of the lift caused by the rotation of the cylinder. On the other hand, for $\tilde{k}_{2}$ the circulation of the vortices shed is kept high and fairly constant for the lower branch, accentuating the fact that the vortices comply importantly with the oscillations of the cylinder as the frequency of oscillation is synchronized with the vortex emission, thus leading to a resonance mechanism of oscillation with the vortex shedding, giving a VIV-type of response.

\subsection{Concluding remarks}

A 2D numerical Lattice Boltzmann Method has been developed to study the dynamical behavior and wake pattern of the fluid flow around a circular cylinder undergoing FIV (for $m^{*}=12.7$ and Reynolds number 100), free to oscillate in the transverse direction with an imposed rotation around its axis. Two different rotating laws have been investigated in particular, first a rotation proportional to the cylinder's position and second a rotation was given proportional to the cylinder's velocity. The main findings are:

- Regarding the case where rotation is proportional to the cylinder's displacement $\left(\theta=\tilde{k}_{1} Y\right)$ it 


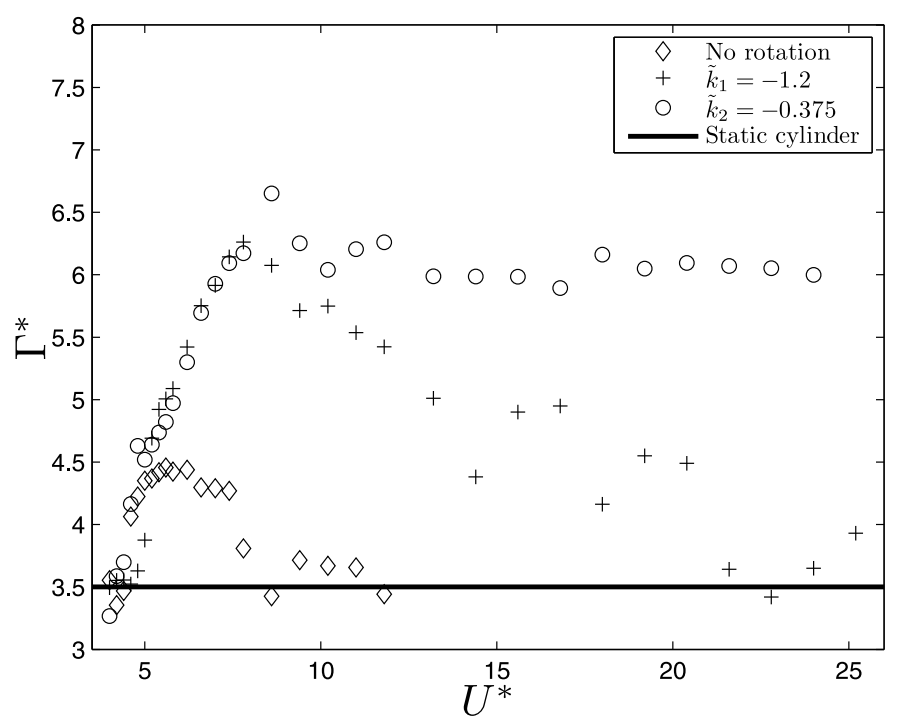

Figure 2.26: Non-dimensional circulation of the vortices shed as a function of the reduced velocity $U^{*}$.

has been found that positive values of $\tilde{k}_{1}$ lead to a reduction in the maximum amplitude of oscillation compared to that of the pure VIV and the transition between the initial-to-lower branch happened for lower values of reduced velocities. Also, the frequency of oscillation was slightly increased and the region of VIV synchronization in terms of reduced velocity was diminished. Therefore, from the practical side this strategy should be sought to protect structures prone to suffering Vortex-Induced Vibrations. On the other hand, negative values of $\tilde{k}_{1}$ increases importantly the maximum value of oscillations as well as it increases the reduced velocity at which such maximum occurs. Also, the region of synchronization is greatly enlarged as $\tilde{k}_{1}$ becomes more negative. In fact, if $\tilde{k}_{1}$ overcomes a certain negative value, the response obtained changes dramatically and a galloping-type of response is obtained, where the amplitude of oscillation increases almost linearly for high values of the reduced velocity. The frequency of oscillation remains constant for high values of reduced velocity and the frequency of vortex shedding increases approximately following the Strouhal law and thus both timescales depart from each other. A large number (increasing with the reduced velocity) of vortices are shed per oscillation cycle. From the practical side, note that negative values of $\tilde{k}_{1}$ could be of interest for energy harvesting purposes as well as to promote mixing.

- A quasi-steady model has been proposed in order to predict the amplitude of oscillation for large negative values of $\tilde{k}_{1}$. When the timescales of the vortex emission and the oscillation period are importantly separated, the force can be considered in a quasi-steady fashion and the $C_{y}$ force coefficient is a function of $Y^{\prime} / U^{*}$ and the rotation rate. For the rotation proportional to the cylinder's displacement, following Eq. 2.25, galloping condition is fulfilled for negative values larger than $\tilde{k}_{1}<-2 C_{D o} / C_{L \alpha}=-1.045$. A linear dependence of the amplitude of oscillation with the reduced velocity is obtained. As $\tilde{k}_{1}$ becomes more negative, the slope of the amplitude oscillation with the reduced velocity increases. The quasi-steady model was compared to the numerical solutions obtained from LBM with really good matching between them.

- For the case where rotation is proportional to the cylinder's velocity $\left(\theta=\tilde{k}_{2} Y^{\prime}\right)$ it has been found that positive values of $\tilde{k}_{2}$ lead to a reduction in the maximum amplitude of oscillation compared to that of the pure VIV and the transition between the initial-to-lower branch happened for lower values of reduced velocities. Also, the frequency of oscillation was slightly increased and 
the region of VIV synchronization was diminished. On the other hand, negative values of $\tilde{k}_{2}$ increases importantly the maximum value of oscillation as well as it increases the reduced velocity at which such maximum occurs. The frequency of oscillation increases significantly with the reduced velocity and the vortex shedding frequency is always synchronized with the frequency of oscillation. Also, the region of synchronization is greatly enlarged as $\tilde{k}_{2}$ becomes more negative. For all values of $\tilde{k}_{2}$, oscillations occur under vortex induced vibrations (bellshaped evolution as a function of the reduced velocity). In order to obtain the same amplitude of oscillation for a given reduced velocity, actuation (say maximum rotation angle) has to be larger than for the case where rotation is proportional to the cylinder's displacement.

- The quasi-steady model presented previously fails when applied to the case of rotating the cylinder proportional to its velocity as the transverse force term associated to the rotation is proportional to the acceleration, thus there is no excitation that overcomes the drag coefficient. This agrees with the LBM results in which all large-amplitude oscillations obtained occur under VIV-type of solution.

- Vortex wakes modes depend mainly on $A^{*}$ and $U^{*} / f^{*}$, as for the case of non-rotating VIV (Williamson and Roshko (1988), Willianson and Govardhan (2004)), but also on the rotation law. This can be deduced from the comparison of vortex wakes modes shown in Fig. 2.14 $(\theta=-1.2 Y)$ at point marked as III $\left(A^{*} \approx 1.45, U^{*} / f^{*} \approx 9.5\right)$ and in Fig. $2.22\left(\theta=-0.375 Y^{\prime}\right)$ at point marked as III $\left(A^{*} \approx 1.45, U^{*} / f^{*} \approx 9.5\right)$. Clearly, vortex wakes modes are very different.

- An analysis of the non-dimensional circulation of the near-wake vortex $\left(\Gamma^{*}\right)$ has shown how in VIV-type response, $\Gamma^{*}$ is high for the entire range of reduced velocities where oscillations are significant. However, when galloping-type responses appear, $\Gamma^{*}$ diminishes progressively with the reduced velocity until saturation close to the static value of $\Gamma^{*}$ (cylinder at rest), see Fig. 2.26. Therefore, large amplitude oscillations are compatible with low values of $\Gamma^{*}$, meaning that the main driving effect is not mainly due to vortex shedding. 


\section{Chapter 3}

\section{Experimental investigation of flow-induced vibrations of a rotating circular cylinder using position and velocity feedback}

\subsection{Introduction}

In the present chapter a forward step from the previous one has been taken. The effect of imposed rotation of a slender elastically-mounted circular cylinder free to oscillate transverse to the incident flow has been investigated experimentally at the "Antonio Barrero Ripoll" recirculating free-surface water channel. The Reynolds number based on the diameter of the cylinder ranges from 1500 to 9000 . Similar to the previous chapter, the rotation direction and rate will be linked to the cylinder's dynamics to determine the effectiveness of this strategy as a way to reduce or enhance oscillations arising from VIV of a circular cylinder. In particular, two feedback rotation laws have been investigated, first the rotation of the cylinder is imposed to be proportional to its own position (that is, $\theta=k_{1} y$, where $\theta$ is the rotation angle, $y$ the cylinder's transverse displacement, and $k_{1}$ is a constant), and secondly, the rotation of the cylinder will be proportional to its velocity of oscillations $\left(\theta=k_{2} \dot{y}\right)$. The effect of such rotating laws on the steady-state amplitude and frequency of oscillations, on how the synchronization regime is modified, as well as on the flow patterns arising in the wake of the cylinder (obtained through PIV measurements) will be evaluated. Emphasis will be placed on the efficient enhancement of the amplitude of oscillation of the circular cylinder, this is, increasing as maximum the amplitude of oscillation with the minimum actuation possible (which may have important implications in the energy harvesting domain). As a secondary result, rotating laws that minimize the amplitude of oscillation will be sought (as a protection mechanism against undesired VIV oscillations).

The numerical investigation carried out in the previous chapter of flow-induced vibrations with imposed rotation was done at a substantially lower Reynolds number (in particular, $R e=100$ ). It provided us with a tool to accessibly study the expected response for the problem in question with the capacity of analyzing efficiently a wide range of parameters involved. However, in many real engineering applications, a 2D simulation at Reynolds 100 might not be accurate enough as engineering structures usually deal with Reynolds number which are at least several orders of magnitude higher than the one investigated in the previous chapter. As will be discussed, there are significant differences between the VIV response at the low-end Reynolds number compared to the medium-to-high Reynolds 
region. These differences will necessarily affect the dynamical response of the flow-induced problem under investigation, thus motivating us to experimentally investigate the problem in question at a Reynolds number large enough.

\subsubsection{Differences in the VIV response emerging as a result of the Reynolds number}

Many parameters affect the expected response of an elastically-mounted circular cylinder free to oscillate in the transverse direction with respect to the incident current undergoing VIV oscillations. Taking into consideration the effect of the Reynolds number on the topological behavior, the VIV response at low enough Reynolds number (such as the numerically investigated in the previous chapter, $R e=100)$ is characterized by a 2 branch curve which presents a maximum amplitude of $A^{*} \approx 0.58$. The branches appearing are usually classified as the initial branch and the lower branch. The initial branch corresponds to the initial reduced velocities where oscillations start. The vortical structure after the body is characterized by a $2 \mathrm{~S}$ mode of vortex emission (single vortices being shed per half cycle of oscillation). After the initial-to-lower branch transition the maximum amplitude is obtained. The lower branch then exhibits a $\mathrm{C}(2 \mathrm{~S})$ mode of vortex emission, where single vortices are shed per half-cycle and downstream they appear to coalesce.

On the other hand, for large enough Reynolds number, the response is characterized by a 3 branch curve. They are classified as the initial, upper and lower branches. The main difference (compared to low Reynolds number response) arises from the upper branch which only appears for high enough Reynolds number. The upper branch appears in the transition between the initial-to-lower branches and it is portrayed by being the region within the VIV curve with largest amplitude of oscillation and happening at a reduced velocity where natural frequency of oscillation and frequency of vortex emission match. With respect to the modes of vortex emission, as similar to the low-end Reynolds number, the initial branch is composed by a $2 \mathrm{~S}$ mode of vortex emission. Differently, the lower branch in this case presents a $2 \mathrm{P}$ mode of vortex emission where pairs of vortices (with opposite rotation direction) are shed per half cycle. The upper branch usually presents a $2 \mathrm{P}_{0}$ mode of vortex emission where pairs of vortices are shed per half cycle of oscillation, but the secondary vortex is much smaller than the main one. This configuration is more efficient to transfer energy to the body. For both cases, maximum amplitude of oscillation occurs right after the initial branch transition, which corresponds to a reduced velocity of $U^{*} \sim \mathrm{St}^{-1}$, where both frequency of oscillation and frequency of vortex emission concur, which is the optimal configuration for a resonance type of fluid-induced vibrations. In relation to the synchronization region, defined as the region where large amplitude oscillations occur, it is mostly determined by the value of reduced mass value $m^{*}$ though to a lesser extent it is also Reynolds dependent. Govardhan and Williamson (2006) observed that not only the Reynolds number determined if a 2 branch or a 3 branch type of response would appear, but also was dependent on the combined mass-damping parameter $m^{*} \zeta$. They approximately described the regions where each of them were expected to appear regarding the values of Reynolds number and the combined mas-damping parameter $m^{*} \zeta$ as in Fig. 3.1.

Superimposed in Fig 3.1 have been placed the configuration studied in the previous chapter ( $R e=100$ and no structural damping), which fell well within the 2 branch response region, and the configuration studied for the present chapter $(R e=3200$ at the peak amplitude with close to zero value of $m^{*} \zeta$ ) which falls in the region of the 3 branch response. Note how the 2 branch response is limited to $A^{*}=0.6$ which is closely related to the maximum value of oscillation expect for the lower branch.

The vortex shedding structures downstream a circular cylinder under forced or free vibrations have been thoroughly investigated and classified in previous studies (Williamson and Roshko (1988), Brika and Laneville (1993); Govardhan and Williamson (2000) or Carberry et al., 2005). The most detailed 


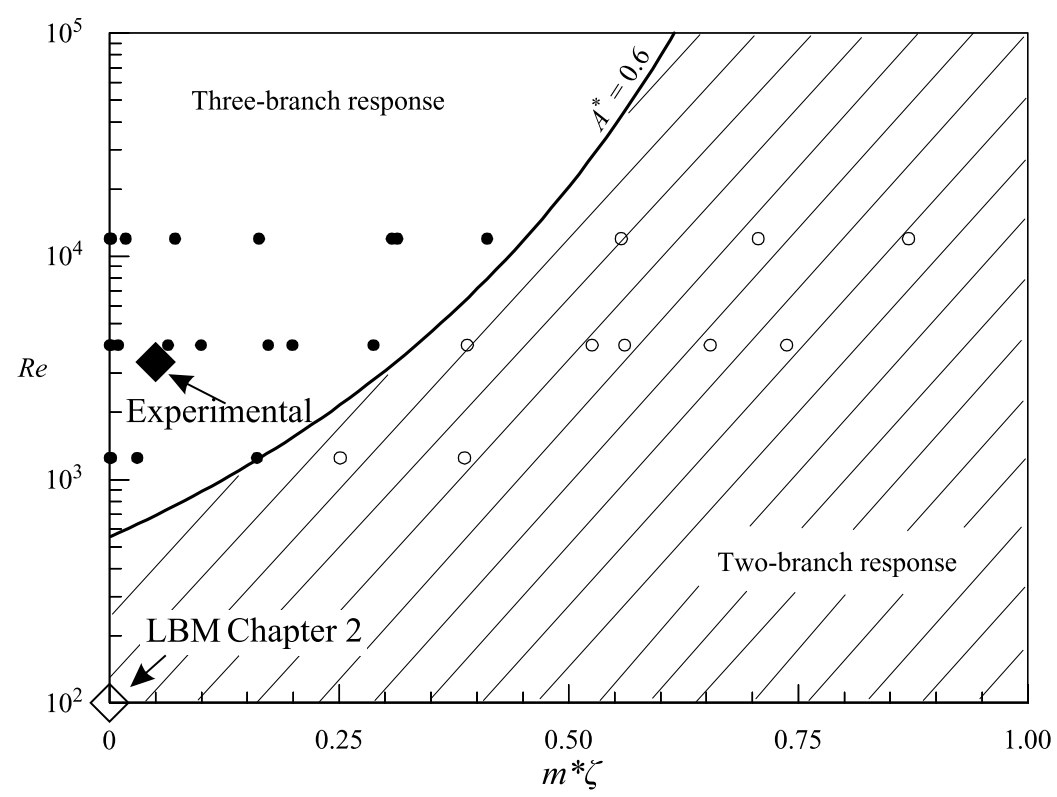

Figure 3.1: Boundary separating the two types of amplitude response (three branch versus two branch) as a function of the combined mass damping parameter $\left(m^{*} \zeta\right)$ and Reynolds number. The line dividing the two regimes is defined by the non-dimensional amplitude of oscillation being $A^{*}=0.6$. It suggests approximately that below $R e \approx 500$ only the two branch response will occur independently of the value of $m^{*} \zeta$ (Govardhan and Williamson, 2006). Superimposed are shown the values of Reynolds number and $m^{*} \zeta$ for the numerical investigation led in the previous chapter $\left(R e=100\right.$ and $\left.m^{*} \zeta=0\right)$ and for the current experimental setup $\left(R e=3200\right.$ at the peak amplitude and $\left.m^{*} \zeta=0.0506\right)$.

map of vortex emission modes for forced vibration has been obtained by Morse et al. (2009) at a Reynolds number of 4000 (Fig 3.2). A VIV curve obtained in the "Antonio Barrero Ripoll" water channel at Reynolds number 3200 (at the peak amplitude) has been superimposed. Note how the initial branch approximately falls in the $2 \mathrm{~S}$ mode of vortex emission, the upper branch in the $2 \mathrm{P}_{0}$ mode and the lower branch to the $2 \mathrm{P}$ mode (this will be showed through PIV visualizations that have been carried out throughout the current investigation).

Reynolds number, apart from determining if the upper branch appears, also importantly influences the maximum amplitude of oscillation. Traditionally it was considered that the main parameter that determined the amplitude of oscillation was the Skop-Griffin parameter $S_{G}=2 \pi^{3} \operatorname{St}\left(m^{*} \zeta\right)$, which takes into account basically for the mass-damping product. The Griffin plot, where the maximum amplitude of oscillation was plotted against the Skop-Griffin parameter, was usually used to predict the maximum expected amplitude. However, as seen in Fig. 3.3 this fit was not completely satisfactory. Govardhan and Williamson (2006) found that in order to better fit all existing results it was necessary to introduce the effect of the Reynolds number obtaining a best fit solution as a function of the Reynolds number and the product $m^{*} \zeta$ as

$$
A_{f i t}^{*}=\left(1-1.12 m^{*} \zeta+0.30\left(m^{*} \zeta\right)^{2}\right) \log \left(0.41 R e^{0.36}\right) .
$$

Big differences in vortex mode shedding between high and low Reynolds number, as well as in the topology of the VIV curve, together to variations in maximum amplitude and 3-dimensional effects appearing at large enough Reynolds encourage us to study the problem experimentally. The rest of the paper is organized as follows, in Section 3.2, the experimental setup is described, including a description of the recirculating water channel where experiments have been performed as well as a description of the Particle Image Velocimetry (PIV) set used to characterize the flow field of the near wake of the oscillating cylinder. Also, validation tests of the water channel are given by comparing 


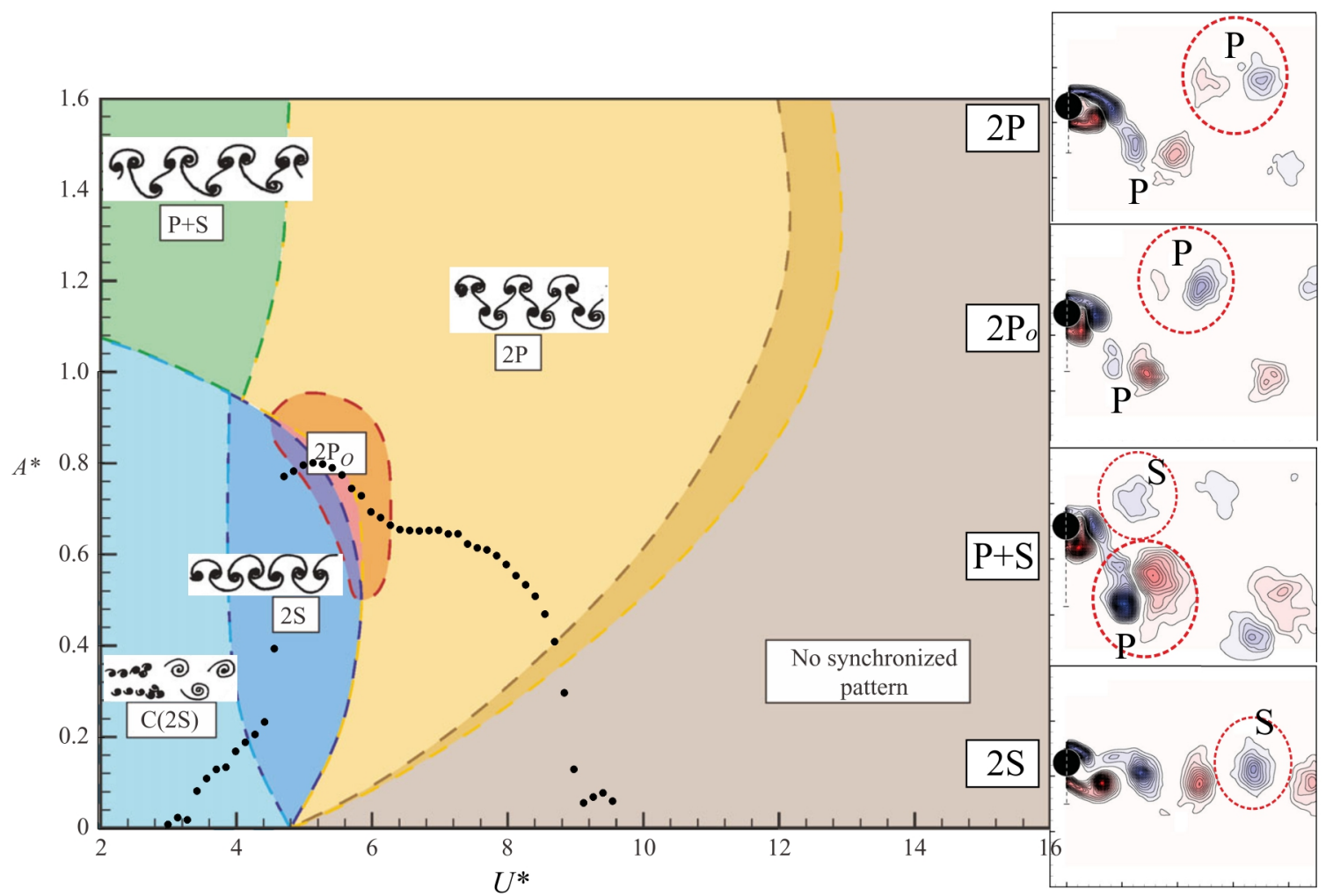

Figure 3.2: Map of vortex-shedding regimes obtained from forced vibrations at $R e=4000$ as a function of the reduced velocity and the reduced amplitude of oscillation. Also, non-dimensional vorticity contours of the main vortex shedding modes have been recovered from Morse and Williamson (2009). Free vibrations of circular cylinder undergoing VIV obtained in the "Antonio Barrero Ripoll" have been superimposed for $R e=3200$ at the peak amplitude and $m^{*}=11.7$.

VIV results to similar ones published in the literature. In Section 3.3, flow-induced vibration results for the cylinder with prescribed rotatory law proportional to the cylinder's position is presented first. Significant changes in the cylinder's response are found. Depending on the sign of the constant of proportionality between rotation and cylinder's displacement, the response can be diminished or significantly enhanced, even a galloping-type response emerges when the forced rotation overcomes a certain level. A quasi-steady theoretical model has been developed which can reasonably predict amplitude of oscillation for these cases (galloping-type response). Right after, the results for the cylinder with a rotation law proportional to the cylinder's velocity is presented. In this case, it is possible to increase or diminish oscillations but the response always has a vortex-induced-type character. For each of the rotation laws investigated, vorticity contour maps obtained through PIV measurements have been presented and discussed. Finally, concluding remarks are presented in Section 3.4 .

\subsection{Experimental setup and validation}

\subsubsection{Experimental setup}

The experiments were carried out in the "Antonio Barrero Ripoll" hydrodynamic water channel of the Escuela Técnica Superior de Ingenieros Aeronáuticos of the Universidad Politécnica de Madrid. It is a free-surface recirculating water channel with controlled inflow conditions at the test section in terms of mean speed, uniformity, and low turbulence. A sketch of the water channel is given in 


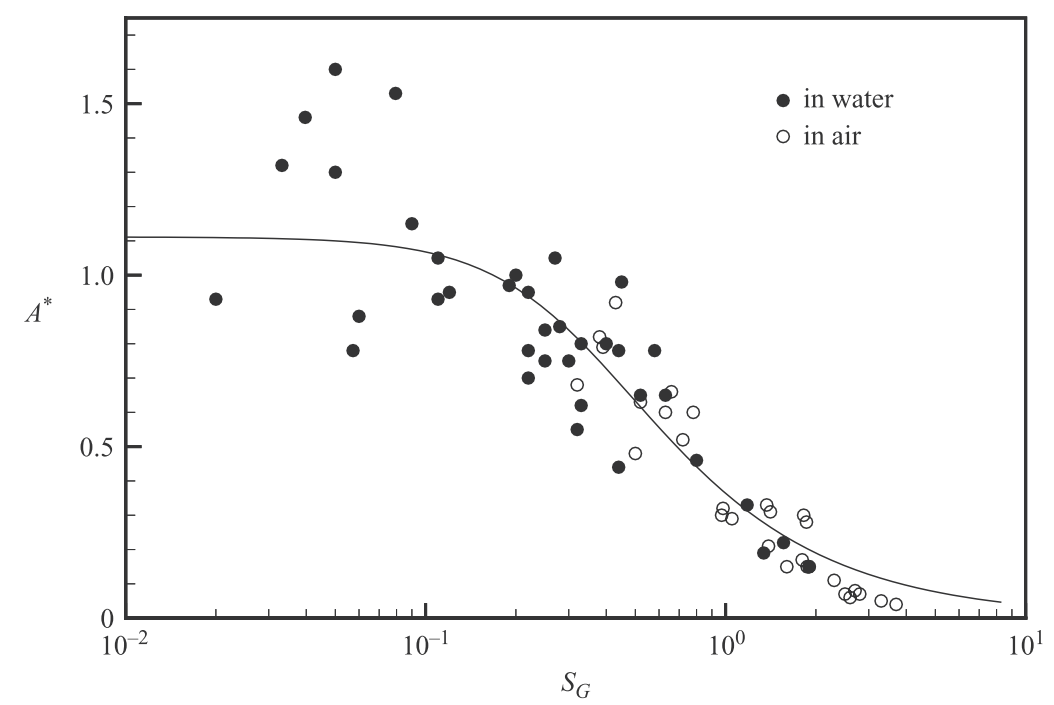

Figure 3.3: The Griffin plot comparing peak amplitude of oscillation versus the Skop-Griffin parameter, Skop and Balasubramanian (1997).

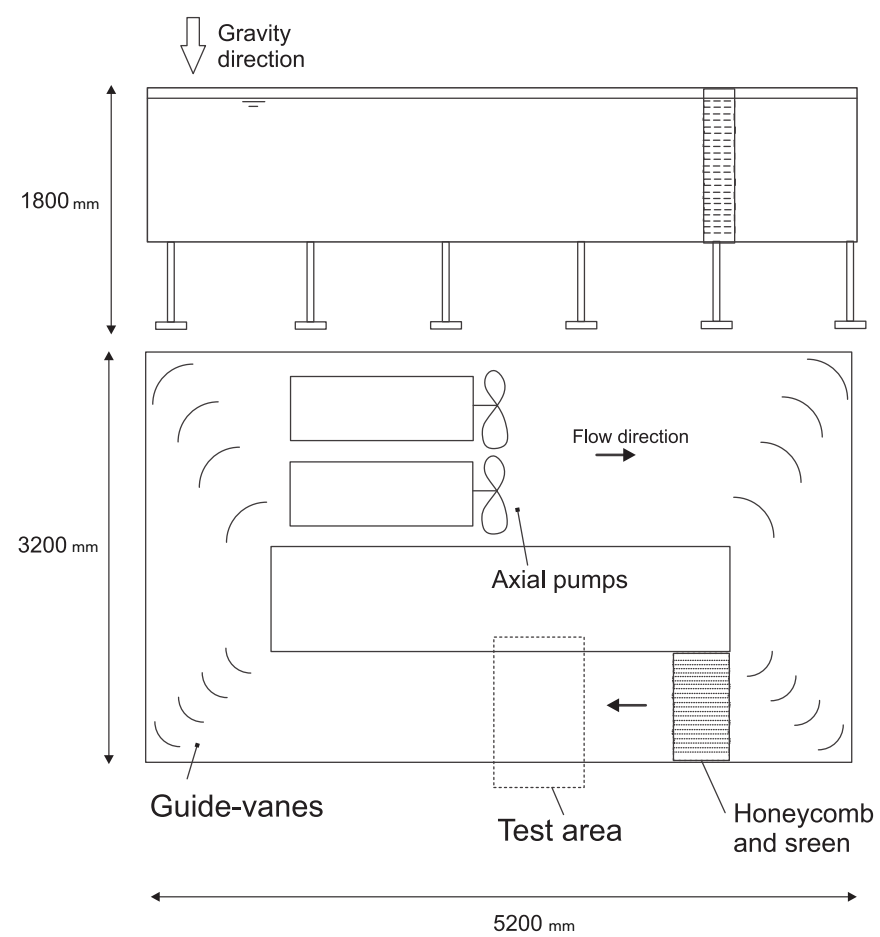

Figure 3.4: Schematic elevation and plan of the free-surface water channel "Antonio Barrero Ripoll".

Fig. 3.4. The water current is driven by two identical axial pumps manufactured by ABS, model RCP $500\left(Q_{\max }=0.69 \mathrm{~m}^{3} / \mathrm{s}, \Delta p_{\max }=13000 \mathrm{~Pa}, P_{\max }=11 \mathrm{~kW}\right)$. The rotation speed of the pumps is regulated by a variable frequency drive from Power Electronics, model SD503942. Guide vanes are placed in the corners of the water channel to guide the flow and reduce pressure-losses. To improve flow quality in the test section, a honeycomb (hexagonally shapped cells with diameter $4.5 \mathrm{~mm}$ and a length to diameter ratio of 12) and a fine screen are located before the entrance of the test section. As known, the honeycomb is a very effective flow straightening device (as shown by Bradshaw and Pankhurst, 1964) and the screen is effective to reduce mean non-uniformities and fluctuations of the streamwise component. 
With respect to the inflow conditions of the water channel test section, the velocity field was measured (without the cylinder mounted) using a Pitot Tube and a differential pressure sensor Druck (model LPM11810-C1SNW-1 with a range 0-20 mbar and a resolution of \pm 0.05 mbar). The instantaneous velocity was obtained through the Bernouilli equation, $u=\sqrt{2 \Delta p / \rho} \mathrm{m} / \mathrm{s}$. The Pitot Tube was mounted in a linear motorized guide (with stepper motors) in order to map a transverse plane perpendicular to the incident flow. The velocity of the water $u(y, z)$ was measured in a $8 \times 8$ matrix of points separated equidistantly between them. Measurements were obtained for different values of the operating frequency of the axial pumps in order to obtain a linear best fit of the incoming water speed as a function of such selected values of operating frequency.
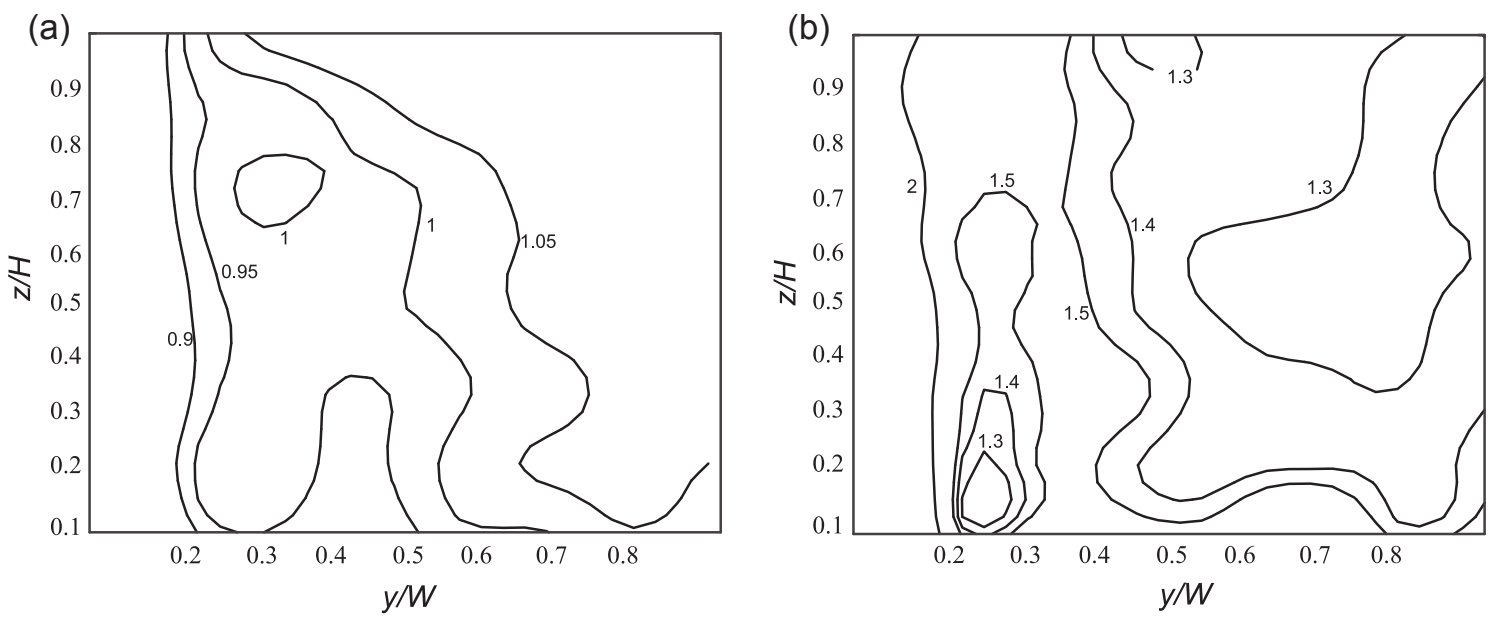

Figure 3.5: (a) Non-dimensional mean velocity contour map. (b) Turbulence intensity level contour map. These maps have been obtained at a temporary and spatially averaged velocity of $U=0.350$ $\mathrm{m} / \mathrm{s}$.

As an example, in Fig. 3.5a, the non-dimensional mean velocity contour map depicted as < $u(y, z)>/ U$, where $<.>$ represents the averaged value in time and $U$ is the spatially and temporary averaged value of the inflow velocity for a value of $U=0.350 \mathrm{~m} / \mathrm{s}$ is shown. As can be observed, the temporary averaged inflow velocity is quite even within the region of interest with variations restrained under $5 \%$ (for values of the transverse direction between $0.2<y / W<0.7$ which gives an effective test window of approximately $450 \mathrm{~mm}$ in width) except for areas next to the walls. In Fig. 3.5b variations of the level of turbulence intensity referred to the main velocity is plotted. The turbulence intensity is defined as $u^{\prime}(y, z) / U$ where $u^{\prime}$ is the root-mean-square of the velocity fluctuations. The turbulence intensity level measured is within the order of magnitude of similar free-surface recirculating water channels (Assi et al. (2009), Govardhan and Williamson (2006) and Radi et al., 2014).

The test section is $3000 \mathrm{~mm}$ in length, $900 \mathrm{~mm}$ in width and the working depth is $830 \mathrm{~mm}$ (A; see Fig. 3.6). The test section is made of glass which allows the flow to be viewed from either side, as well as from the bottom, allowing to perform flow visualizations through PIV measurements. A circular cylinder (B), made of aluminium, of $25 \mathrm{~mm}$ of diameter $D$ and immersed length $L$ of $430 \mathrm{~mm}$, was attached through a bearing (C) to the shaft of a servomotor (D) fixed to the free end of a doubleblade elastic system, following the arrangement introduced in Assi et al. (2006). The elastic system was made up of with two parallel rigid aluminium blocks (E), coupled to a pair of thin spring-steel 


\section{(a)}

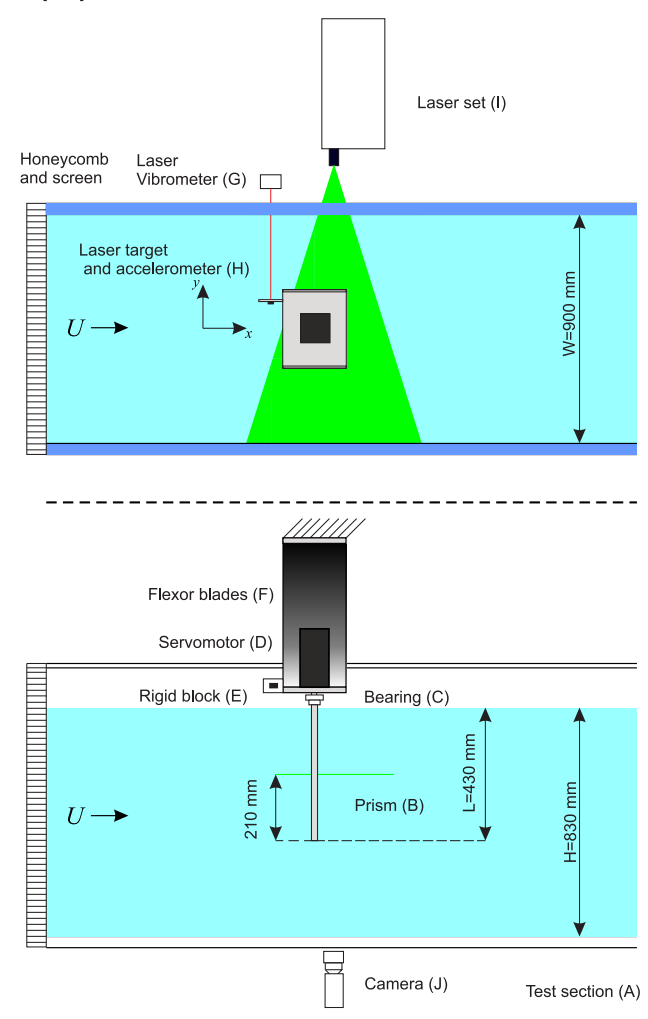

(b)

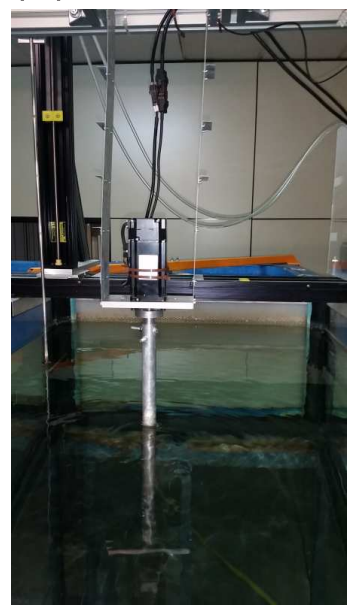

(c)

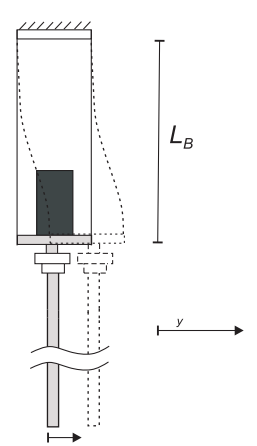

Figure 3.6: Sketch of the experimental set-up (dimensions are in millimeters) of the water channel. (a) Schematical top view and side view of the water channel. (b) Photograph of a close view of the elastic system. (c) Detail of the elastic system's deformation when a translation in the transverse direction is applied.

flexor blades (F). The elastic system not only acts as the cylinder support, but also provides the linear restoration response. In this way, the cylinder is restricted to oscillate in the transverse direction to the flow $(y)$ with low mechanical damping. Figure $3.6 \mathrm{~b}$ is a detailed view of the flexors and elastic prism system including the servomotor fixed to it, where the experiments were carried out. The translation and acceleration of the circular cylinder were measured by a non-contact laser vibrometer $(\mathrm{G})$ from Aquity AR500-500 (range $500 \mathrm{~mm}$, resolution $0.05 \mathrm{~mm}$ ) and by an accelerometer $(\mathrm{H})$ from Measuring Specialities (range $\pm 2 \mathrm{~g}$, resolution $2 \mathrm{mg}$ ). The flow speed $(U)$ was varied between $75 \mathrm{~mm} / \mathrm{s}$ and 450 $\mathrm{mm} / \mathrm{s}$, which gives a Reynolds number range of $R e=U D / \nu=1500-9000, \nu$ being the kinematic viscosity of the water.

An AC servomotor 60ST-M013330C from MIGE, with a peak torque of $3.9 \mathrm{Nm}$ and a maximum rated speed of $3000 \mathrm{rpm}$ was used. To measure the angle of rotation, the servomotor includes a photoelectric encoder with resolution of $0.036^{\circ}$. To impose the rotation law, a signal command was sent to the servomotor controller. Depending on the rotation law, this signal command was proportional either to the cylinder's position (measured with the laser vibrometer) or to the cylinder's velocity (obtained by integration of the accelerometer's signal). The time delay between the servomotor response and the signal command was under $5 \mathrm{~ms}$, which is small enough compared to the characteristic oscillation times during the experiments (a maximum phase lag of $1.5^{\circ}$ ). Calibration experiments were performed to determine such time delay. A major challenge was to minimize the unbalance of the rotating cylinder to avoid undesirable effects on the dynamics of the wake. The cylinder was connected to the shaft of the servomotor through a bearing manufactured at our installations. Before 
each run, parallelism of the cylinder as well as dynamic balancing was checked (through measurements of the accelerometer installed).

Output signal used to control the servomotor was generated through an analogue output module $( \pm 10 \mathrm{~V}, \mathrm{NI}-9264)$. All data (position, acceleration, rotation angle) was recorded and registered using an analogue input module $( \pm 10 \mathrm{~V}, \mathrm{NI}-9201)$ and a Compact-Rio microprocessor from National Instruments (NI cRio-9024) with sampling frequency of $200 \mathrm{~Hz}$ for each parameter combination of flow speed velocity and rotation law used for a total of 16 minutes. The first 6 minutes were spent on achieving steady state conditions while the remaining 10 minutes were used to recording. After that, the water speed was increased and the measurement procedure repeated.

\subsubsection{Data reduction and rotation laws evaluated}

For each run, the instantaneous position $y(t)$, acceleration $\ddot{y}(t)$ and rotation angle $\theta$ of the cylinder was directly measured and registered. The dynamical equation of the cylinder and the rotation laws employed are respectively:

$$
\begin{gathered}
m \ddot{y}+c \dot{y}+k y=-m_{A} \ddot{y}+\frac{1}{2} \rho U^{2} D L C_{y}, \\
\theta=k_{1} y \quad \text { or } \quad \theta=k_{2} \dot{y},
\end{gathered}
$$

where $m$ is the total oscillating mass, $c$ the mechanical damping constant, $k$ the stiffness of the system, and $k_{1}$ and $k_{2}$ are feedback constants which relate the rotation to be imposed on the cylinder and the displacement/velocity of the cylinder respectively. Both $c$ and $k$ were measured in free decay tests in still air (empty water channel). Regarding the right hand side of Eq. 3.2a, note that the fluid force has been split into two terms, an added mass term $\left(-m_{A} \ddot{y}\right)$ and a viscous one $\left(\rho U^{2} D L C_{y} / 2\right)$, as suggested in Govardhan and Williamson (2000); $\rho$ is the fluid density, and $C_{y}$ a dimensionless fluid force coefficient. This makes sense in order to model appropriately the fluid force exerted on the cylinder for zero flow speed.

Eq. 3.2b shows the two rotation laws that have been tested in order to determine how such rotation affects VIV response of a circular cylinder; in particular, (i) the cylinder is rotated proportionally to its displacement and (ii) the cylinder is rotated proportionally to its velocity.

The equation of the dynamics of the cylinder and the rotation laws prescribed can be made dimensionless introducing $D$ and $f_{N}^{-1}=\left(\left(m+m_{A}\right) / k\right)^{1 / 2}$ as representative length and time scales. This gives

$$
\begin{gathered}
Y^{\prime \prime}+4 \pi \zeta Y^{\prime}+4 \pi^{2} Y=\frac{2 U^{* 2}}{\pi\left(m^{*}+C_{A}\right)} C_{y}, \\
\theta=\tilde{k}_{1} Y \quad \text { or } \quad \theta=\tilde{k}_{2} Y^{\prime},
\end{gathered}
$$

where $Y=y / D, \zeta=c /\left(2\left(k\left(m+m_{A}\right)\right)^{1 / 2}\right)$ is the damping ratio, $m^{*}=4 m /\left(\pi \rho D^{2} L\right)$ is the mass ratio, $C_{A}$ is the potential added mass coefficient $\left(C_{A}=1.0\right.$ for circular cylinder $), \tilde{k}_{1}=D k_{1}$ and $\tilde{k}_{2}=f_{N} D k_{2}$ are the non-dimensional feedback constants, and 'stands for differentiation with respect non-dimensional time $t^{*}=f_{N} t$.

A usual method of analysis employed for the study of FIV problems is based on the introduction of time-averaged quantities (Sarpkaya, 2004). In this sense the following variables were computed from each run: (i) the steady-state normalized maximum amplitude of oscillation $A^{*}=A_{10} / D$, where $A_{10}$ denotes the maximum $10 \%$ peaks of oscillation for each run, (ii) the normalized frequency of oscillations $f^{*}=f / f_{N}$ where the frequency of oscillations $f$ was computed from Fast Fourier Transform of $y(t)$. 


\subsubsection{PIV measurements}

Particle Image Velocimetry (PIV) is a technique for quantitative flow visualization. It is based on the measurement of the displacement of small light scattering particles that are carried by the flow under consideration. Particles have to be small enough to reliably follow the flow motion without altering the fluid properties or disturbing the flow, but on the other hand they should be able of scattering enough light to get recorded by a camera. The light source needed to illuminate the tracer particles is provided by a thin light-sheet produced by a pulsed light source (normally a double-head pulsed laser system). The particles scatter the light and a digital imaging device (such as a CCD camera) records them in two subsequent image frames which can be correlated to derive the particles displacement. In the present experimental setup, a simplified one-camera system has been used which only allows to determine two velocity components within the laser sheet. The optical axis has to be aligned in the direction normal to the light-sheet plane.

The PIV set used to characterize the flow field of the near wake of the cylinder was from Dantec Dynamics. Flow illumination was provided by a pulsed Nd:YAG $800 \mathrm{~mJ}$ laser with a wavelength of $532 \mathrm{~nm}$. Each laser pulse lasted for $4 \mathrm{~ns}$ which is small enough to see the particles as dots because the displacement of the particle in exposure times of $4 \mathrm{~ns}$ is much smaller than the size of the particle for the characteristic flow velocities for the present experimental setup. The architecture of the laser under consideration consists of two separated lasers firing independently at the required pulse separation. The major limitation of the Nd:YAG-based systems is that its repetition rate is between 10 and 50 $\mathrm{Hz}(15 \mathrm{~Hz}$ in this case) which may pose serious drawbacks for time-resolved experiments. In the later, this specific question will be treated.

Images were taken using a Dantec Dynamics Flow Sense 2ME camera with a resolution of 1600 $\times 1200$ pixels with pixel size $7.4 \mu \mathrm{m}$. Data transmission was made directly to the PC RAM via a PCIe-1427 image acquisition board which allows for an information transfer larger than $200 \mathrm{MB}$ per second. At a sampling frequency of $15 \mathrm{~Hz}$, the camera transferred 15 pair of images per second which led to an effective data transfer of $110 \mathrm{MB}$ per second for a bit depth of $16 \mathrm{bit} / \mathrm{pixel}$. CPU RAM reserved for data acquisition was $4 \mathrm{~GB}$, so a maximum of 537 pair of images per test batch could be stored, which was equivalent to a $35 \mathrm{~s}$ of images recording.

The camera lens was a Zeiss Makro-Planar T 2/50 mm ZF. The laser sheet intercepts the circular cylinder perpendicularly to its axis at a height of $210 \mathrm{~mm}$ from its bottom end. After passing side walls, it was verified that the thickness of the laser sheet was smaller than $1 \mathrm{~mm}$. The camera was set under the water channel perpendicular to the laser sheet. Synchronization between image capturing and flow illumination was carried out using a timer box provided by Dantec Dynamics through a National Instruments Board PCI-6602 timer board. Laser pulse, image acquisition, synchronization of the components and the analysis was carried out using the Dynamic Studio Dantec software.

Regarding flow seeding, Hollow Glass Spheres with a mean diameter of $10 \mu \mathrm{m}$ (HGS-10) was used (size distribution 2-20 $\mu \mathrm{m}$ and density $\rho_{p}=1.1 \mathrm{~g} / \mathrm{cm}^{3}$ ). The desired concentration of particles per window has been calculated to be at least 10 particles per window. That means that a single particle cannot be tracked because the mean displacement is larger than the mean distance between two adjacent particles. One needs to rely on statistics to identify the most probable match of particle images between to consecutive frames. In order to determine the characteristic time response of the particles, the equation of motion of a small spherical particle immersed in a fluid flow is given by:

$$
\frac{\pi}{6} D_{p}^{3} \rho_{p} \frac{d \mathbf{u}_{p}}{d t}=\mathbf{F}_{f}+\underbrace{\frac{\pi}{6} D_{p}^{3} \rho_{p} \mathbf{g}}_{\text {Gravity force }},
$$

where " $p "$ subindices indicates that the magnitude is referred to the particles. From Maxey and Riley (1983) and following the analysis in Serrano (2013), assuming that the quasi-steady particle response 
is dominated by viscosity and that the characteristic flow scale is much larger than the particle size, the fluid force $\mathbf{F}_{f}$ on the particle can be expressed as

$$
\begin{gathered}
\mathbf{F}_{f}=\underbrace{3 \pi \mu D_{p}\left(\mathbf{u}-\mathbf{u}_{p}\right)}_{\text {(1) Stokes Resistance }}+\underbrace{\frac{\pi}{12} D_{p}^{3} \rho \frac{d\left(\mathbf{u}-\mathbf{u}_{p}\right)}{d t}}_{(2) \text { Virtual Mass }}+ \\
\underbrace{\frac{3}{2} D_{p}^{2} \sqrt{\pi \rho \mu} \int_{0}^{t} \frac{\frac{d\left(\mathbf{u}-\mathbf{u}_{p}\right)}{d t}}{\sqrt{t-\tau}} d \tau}_{\text {(3) Basset }}-\underbrace{\frac{\pi}{6} D_{p}^{3} \rho_{p} \nabla\left(\frac{p}{\rho_{p}}\right)}_{\text {(4) Pressure Far Field }}
\end{gathered}
$$

Performing an order of magnitude analysis by comparing each term with the Stokes one, one finds that

$$
\begin{gathered}
\frac{(2)}{(1)} \sim \frac{D_{p}^{2}}{36 \nu t_{c}}, \\
\frac{(3)}{(1)} \sim \sqrt{\frac{D_{p}^{2}}{4 \pi \nu t_{c}}}, \\
\frac{(4)}{(1)} \sim \frac{D_{p} \mathrm{u}}{18 \nu} \frac{\mathrm{u}}{\left|\mathbf{u}-\mathbf{u}_{p}\right|} \frac{D_{p}}{l_{w}}, \\
\frac{D_{p}^{2} g}{\text { Gravity force }} \sim \frac{\rho_{p}}{18 \nu\left|\mathbf{u}-\mathbf{u}_{p}\right|},
\end{gathered}
$$

where $t_{c}$ is a characteristic time of the velocity fluctuations in the water, and $l_{w}$ represents a characteristic length of the water flow. All the terms in the previous expressions should be negligible for small enough particle size. In particular, the virtual mass and the Basset terms are negligible if the flow around the particle is dominated by viscosity $\left(R e_{p} \ll 1\right)$. The pressure far field generates velocity differences of the order $R e_{p} D_{p} / l_{w} \ll 1$ of the water velocity, whereas the gravity sedimentation term generates velocity differences of the order of $\frac{D_{p}^{2} g}{18 \nu \mathrm{u}} \frac{\rho_{p}}{\rho}$ of the water velocity. Thus, for $D_{p} / l_{w} \ll 1$, $R e_{p} \ll 1$ and $\frac{D_{p}^{2} g}{18 \nu \mathrm{u}} \frac{\rho_{p}}{\rho} \ll 1$, the Stokes term is the dominant one and Eq. 3.5 is reduced to

$$
\frac{1}{18} \frac{D_{p}^{2}}{\nu} \frac{\rho_{p}}{\rho} \frac{d \mathbf{u}_{p}}{d t}=\left(\mathbf{u}-\mathbf{u}_{p}\right) .
$$

For this case, the particles act as a linear first order low pass filter with characteristic cut-off frequency $f_{c}=\frac{\rho}{\rho_{p}} \frac{9 \nu}{\pi D_{p}^{2}} \approx 3 \cdot 10^{6} \mathrm{~Hz}$ which highly exceeds the characteristic frequencies that can be measured through the PIV set used.

PIV measurements were carried out with the laser sheet perpendicular to the circular cylinder at a constant height of $210 \mathrm{~mm}$ from the bottom end of the prism with the camera situated under the water channel (orientated perpendicular to the laser sheet, see Fig. 3.6a). The field of view of the camera corresponds approximately to a rectangle of $9 D \times 6.75 D$ which corresponds to physical dimensions of $225 \mathrm{~mm} \times 169 \mathrm{~mm}$ containing $1600 \times 1200$ pixels with a pixel size of $7.4 \mu \mathrm{m}$. The cylinder is situated at the centerline of the y-axis of the camera's field of view and one diameter distance downstream from the inlet boundary wall, thus focusing in the near wake after the circular cylinder and sampling of the flow field was carried out at a frequency of $15 \mathrm{~Hz}$.

With regard to the post-processing of the images, each sample of the flow field was generated through an adaptive cross-correlation from the images obtained from two consecutive laser pulses. In cross-correlation, the recorded image is divided into interrogation windows and within each window a cross-correlation matching in the two consecutive pair of images is done. Let us assume that $I_{1}(x, y)$ and $I_{2}(x, y)$ is the light intensity map of two consecutive snapshots. The cross-correlation function 
obtained in a specific measurement window centered at $\left(x_{0}, y_{0}\right)$ is obtained as (see Fig. 3.7)

$$
R_{I}\left(x_{0}, y_{0} ; \delta x, \delta y\right)=\int_{-\Delta x / 2}^{\Delta x / 2} \int_{-\Delta y / 2}^{\Delta y / 2} I_{1}\left(x_{0}+\varsigma, y_{0}+\eta\right) I_{2}\left(x_{0}+\varsigma+\delta x, y_{0}+\eta+\delta_{y}\right) d \eta d \varsigma .
$$

The mean displacement of the particles in the window under consideration is obtained as the values of $\delta x$ and $\delta y$ that maximize the cross-correlation function in Eq. 3.8.

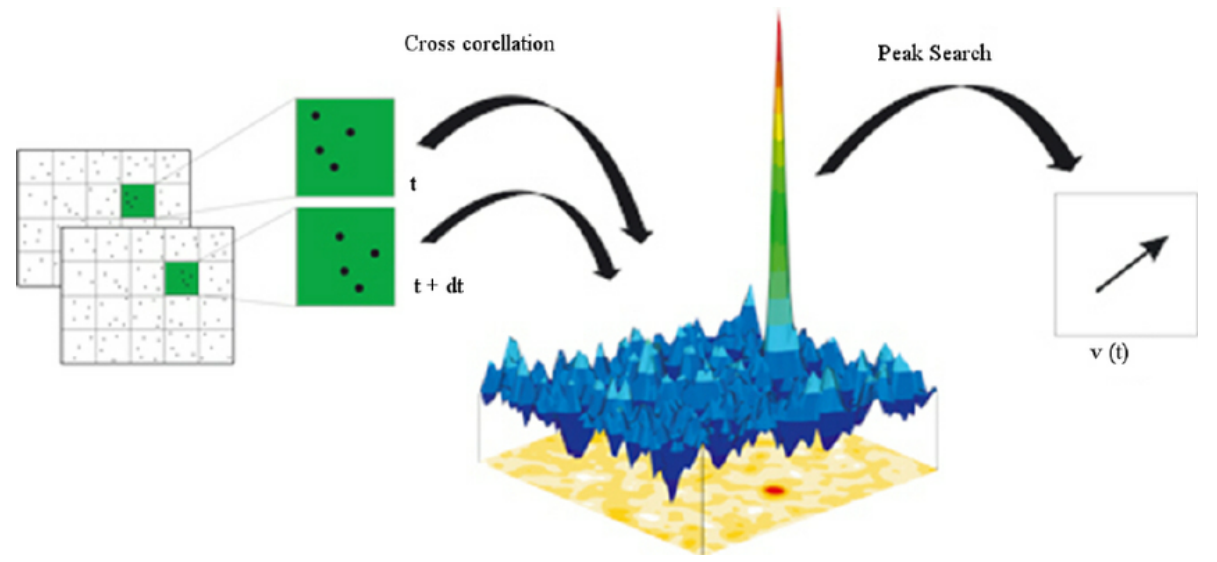

Figure 3.7: Histogram analysis for finding the most probable displacement.

In particular, two consecutive laser pulses fired were separated $3 \mathrm{~ms}$ in time. For the case of highest reduced velocity analyzed $\left(U^{*}=15\right)$, the mean flow velocity was $0.38 \mathrm{~m} / \mathrm{s}$, thus, a particle would travel around $1 \mathrm{~mm}$ between two consecutive laser pulses (which is quite smaller than the characteristic length of the problem $D=25 \mathrm{~mm}$ ). Each PIV area was divided into smaller sub-interrogation areas (adaptive cross-correlation) of $128 \times 128$ pixels and through a parallel self-consistent re-computation of the flow field in successive interrogation areas of $64 \times 64$ and $32 \times 32$ pixels (or $4.5 \mathrm{~mm} \times 4.5 \mathrm{~mm}$ ), with $50 \%$ overlapping, spatial resolution refinement was obtained (final computations were consistent with the flow fields obtained for larger windows). The final spatial resolution of the flow field for the experimental tests was of the order of $2 \mathrm{~mm}$, which is considered to be sufficient to obtain qualitative information of the near wake vortex shedding structures. Post-processing analysis was performed through Dynamic Studio Dantec software and further post-processing methods were performed when images were analyzed including low-pass and high-pass filtering, histogram equalization, $\min / \max$ filtering to name a few.

A last note regarding spatial resolution needs to be mentioned; the spatial resolution of the PIV data in the z-direction is given by the depth of field of the PIV receiving optics (Adrian and Westerweel, 2011):

$$
\delta z=2 f d_{d i f f} m(1+m) \sim 5500 \mu \mathrm{m},
$$

where $f$ is the f-number of the camera's receiving optics, $m$ is the scale factor between the real and image planes and $d_{d i f f}=2.44 f \lambda(1+1 / m) \sim 3 \mu \mathrm{m}$ accounts for diffraction effects. The spatial resolution in the z-direction is not completely satisfactory and it is caused by the large $m$ number of the scale factor needed in order to be able to observe the vortical structures appearing. However, the z-resolution is not a main drawback as only particles visible within the laser-sheet are illuminated.

With regard to time resolution, large scale vortices have a characteristic timescale (related to the Strouhal number, $\mathrm{St}=f_{\mathrm{St}} D / U$ ) of 1 to $3 \mathrm{~Hz}$ for the range of reduced velocities under consideration, which is roughly 5 to 15 times lower than the acquisition timescale $(15 \mathrm{~Hz})$. The recording sequence consisted of 300 frames (lasting $20 \mathrm{~s}$ ) which is equivalent to approximately 20 oscillation cycles of the circular cylinder. The measurement process was repeated 3 times for each set of parameters 
investigated.

\subsubsection{Validation test}

To validate the experimental set-up and to obtain reference data for comparison, a non-rotating VIV circular cylinder curve was obtained. The circular cylinder had a diameter of $D=25 \mathrm{~mm}$ and submerged length of $L=430 \mathrm{~mm}$ (aspect ratio $L / D=17.2$ and blockage ratio $2.7 \%$ ). From the structural point of view, the mass ratio was $m^{*}=11.7$ and damping $\zeta=0.0043$ (yielding a massdamping ratio $\left.m^{*} \zeta=0.0506\right)$. The Reynolds number at the peak of the amplitude curve response was $R e=3200$.

Fig. 3.8 shows the measured VIV response in terms of $A^{*}\left(A_{10}^{*}\right)$ as a function of the reduced velocity $U^{*}$. All measurements have been made with increasing water speed. For comparison purposes, results from Khalak and Williamson (1999), Assi et al. (2006) and Klamo (2007) are also given. The mass ratio of Khalak and Williamson (1999) $\left(m^{*}=10.1\right)$, Assi et al. (2006) $\left(m^{*}=8.1\right)$ and Klamo (2007) $\left(m^{*}=6.5\right)$ are comparable to our final setup $\left(m^{*}=11.7\right)$, while the mass-damping parameters were $m^{*} \zeta=0.017,0.016,0.01$ respectively, which are smaller to the mass-damping ratio of the present study $\left(m^{*} \zeta=0.0506\right)$. The Reynolds number range of all of the cases are similar. In particular, the Reynolds number at the amplitude's curve peak was for Khalak and Williamson (1999) $R e \approx 5000$, Assi et al. (2006) $R e \approx 5500$, Klamo (2007) $R e \approx 2600$ and $R e \approx 3200$ for the present VIV test.

As can be seen, all VIV responses present three distinctive branches which correspond to the initial, upper and lower branch respectively. As described by Govardhan and Williamson (2006) the VIV curve show such 3 distinctive branches for high enough Reynolds number and low enough massdamping parameter. The transition between the initial and upper branch happens at $U^{*} \approx 4.5$ and a distinctive lower branch can be observed between $U^{*} \approx 6$ and $U^{*} \approx 9$ where desynchronization occurs. With respect to the maximum amplitude obtained, differences appear mainly due to the effect of the Reynolds number. This phenomenon was studied in Govardhan and Williamson (2006) where a 'modified Griffin plot' was introduced and a best-fit for a wide range mass-damping and Reynolds was given as $A_{f i t}^{*}=\left(1-1.12 m^{*} \zeta+0.3\left(m^{*} \zeta\right)^{2}\right) \log _{10}\left(0.41 R e^{0.36}\right)$ which for our experiment setup yields a theoretical maximum amplitude of oscillation of $A_{f i t}^{*}=0.82$ which agrees closely to the maximum amplitude measured of $A^{*}=0.8$.

With regard to the end conditions, no attached end-plate was used (effects of end conditions are reviewed in Morse et al., 2008), which leads to a less distinctive upper/lower transition (similar to those from Klamo (2007), Assi et al., 2006). The decision to not use end-plate was in order to avoid undesired effects when rotating the cylinder where small misalignments lead to big damping effects when the cylinder is rotated.

The overall agreement is good. As can be seen, all VIV amplitude response present slight differences which can be reasonably explained from different experimental conditions listed previously. Other important factors that alter the VIV response, and could explain some differences, includes the inflow properties such as its turbulence or uniformity.

\subsection{Results}

The effects of rotating the cylinder on its dynamical behaviour (namely the amplitude and frequency of oscillation) is here presented. Two strategies are proposed and will be compared mainly focusing on the enhancement in oscillations' amplitude (energy harvesting, favoring mixing of the flow, etc.) or reduction in the oscillations (protection of the engineering structures against VIV). To better understand the mechanisms underlying the oscillations of the cylinder, PIV (Particle Image Velocimetry) visualizations of the near wake are also presented. 


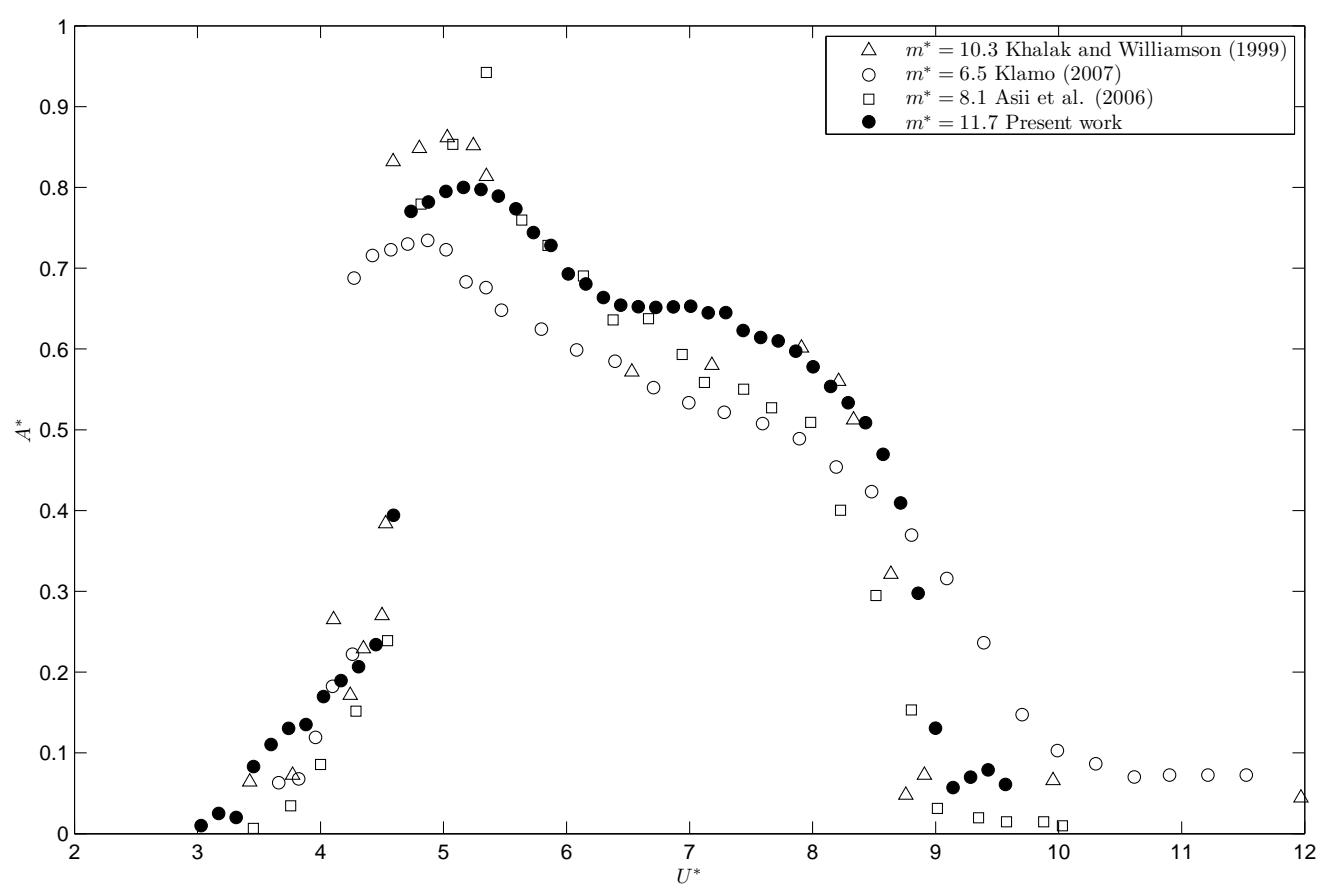

Figure 3.8: Comparison of experimental results for the normalized amplitude variation with reduced velocity. Triangles are from Khalak and Williamson (1999) $\left(m^{*}=10.3, \zeta=0.0017\right)$, open circles are from Klamo (2007) $\left(m^{*}=6.5, \zeta=0.0015\right)$, squares are from Assi et al. $(2006)\left(m^{*}=8.1, \zeta=0.0020\right)$ and solid circles stand for present results $\left(m^{*}=11.7, \zeta=0.0043\right)$.

\subsubsection{Rotation proportional to position}

Firstly, the effect of rotating the cylinder along its axis proportionally to the position of the prism will be studied.

$$
\theta=\tilde{k}_{1} Y
$$

where $\tilde{k}_{1}$ is a non-dimensional feedback constant that relates the position of the prism with the rotation angle of the cylinder along its axis.

\subsubsection{Cylinder dynamics}

Due to the character of the rotation imposed, symmetry is not broken and oscillations take place around the equilibrium position $(Y=0)$ for all values of $U^{*}$ and $\tilde{k}_{1}$. Thus, the cylinder's response can be described by its main frequency of oscillation $f^{*}=f / f_{N}$ and the dimensionless amplitude $A^{*}=A_{10} / D$ of the cylinder's steady-state oscillations.

Fig. 3.9 shows the variation of the amplitude of oscillation for different values of $\tilde{k}_{1}$. Positive values of $\tilde{k}_{1}$ lead to a reduction of the maximum amplitude of oscillation as well as a reduction of the region of $U^{*}$ where the cylinder undergoes large amplitude oscillations. However, as can be seen between $\tilde{k}_{1}=1.25$ and $\tilde{k}_{1}=2.5$, the reduction in amplitude of oscillation is not as significant (Fig. 3.9a). If $\tilde{k}_{1}$ is further increased, the picture does not vary much (regarding amplitude of oscillation, or synchronization region). With respect to the different branches of the VIV curve, the transition between the initial and upper branch is anticipated slightly. On the other hand, the differentiation between upper and lower branches is increasingly lost.

For negative values of $\tilde{k}_{1}$, the picture is quite different (see Fig. $3.9 \mathrm{~b}$ ) and two different responses 

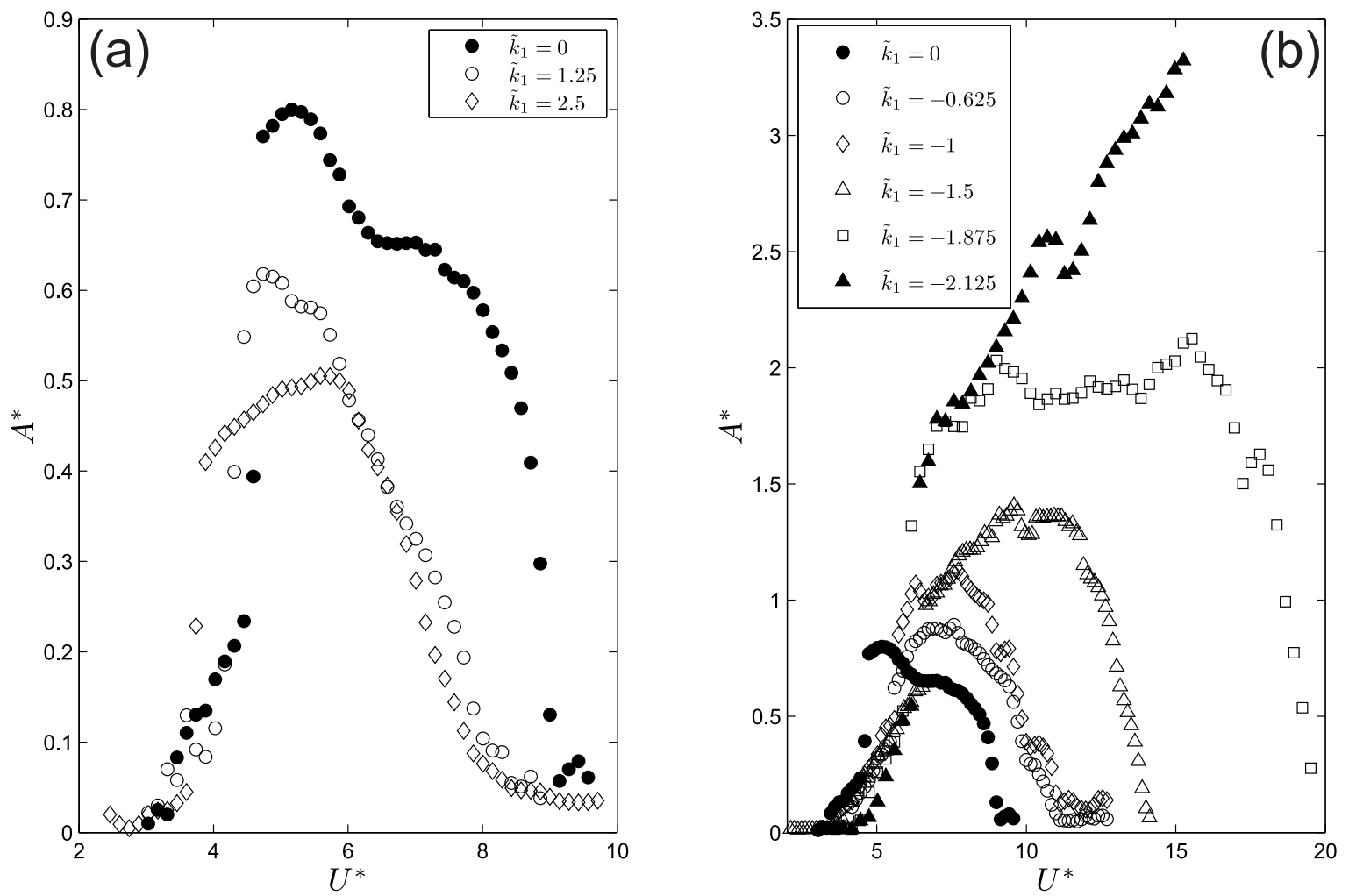

Figure 3.9: (a) Maximum non-dimensional amplitude oscillation as a function of the reduced velocity for positive values of the rotating parameter $\tilde{k}_{1}$ and (b) for negative values of $\tilde{k}_{1}$.

can be observed. For $-1.8<\tilde{k}_{1}<0$, the response amplitude exhibit in all cases a bell-shaped evolution as a function of the reduced velocity which resembles the classical VIV response. This suggests that similar to VIV, the vibrations occur under a wake-body synchronization mechanism. As $\tilde{k}_{1}$ becomes more negative oscillations are more intense, the range of synchronization is enlarged, the reduced velocity at which maximum amplitude, $A_{\text {max }}^{*}$ is achieved is delayed as well as the initial/upper branch transition. A different behavior has been found when $\tilde{k}_{1}<-1.8$. As can be observed, for $\tilde{k}_{1}=-2.125$, just after the initial-to-lower transition $A^{*}$ seems to adopt a quasi-linear dependence with the reduced velocity, which resembles the dependence of galloping-type instability where motion-induced forces are predominant, in contrast to VIV where fluid forces are mainly driven by vortex shedding. This point will be further investigated in the following section. $\tilde{k}_{1}=-1.875$ shows an intermediate behavior, after the initial/upper branch transition, the amplitude response curve adopts a linear trend with the reduced velocity, but at $U^{*} \approx 10$ it loses such trend obtaining an almost constant amplitude of oscillation for increasing $U^{*}$ until large amplitude oscillation cease abruptly at $U^{*}=20$.

In Fig. 3.10, the normalized frequency of oscillations $f^{*}$ is plotted as a function of the reduced velocity for different values of $\tilde{k}_{1}$. Due to the large value of the reduced mass, variation of $f^{*}$ with $U^{*}$ is relatively small. In the initial branch the frequency is lowered slightly and then increases linearly with $U^{*}$. For $\tilde{k}_{1}>0$ the slope of the frequency variation with $U^{*}$ is significantly increased. On the other hand, for negative values of $\tilde{k}_{1}$ (up to $\left.\tilde{k}_{1}>-1.8\right) f^{*}$ is lowered and the slope with $U^{*}$ is smaller. Importantly, for large negative values of $\tilde{k}_{1}$ (i.e. $\left.\tilde{k}_{1}<-1.8\right) f^{*}$ reaches a near constant value after $U^{*} \approx 10$. This result reinforces the idea that for large negative values of $\tilde{k}_{1}$ the cylinder undergoes galloping-type oscillations. Seemingly, the initial/upper branch transition branch occurs near $f^{*}=1$ for all values of $\tilde{k}_{1}$ (similar to the classical VIV response). 
(a)

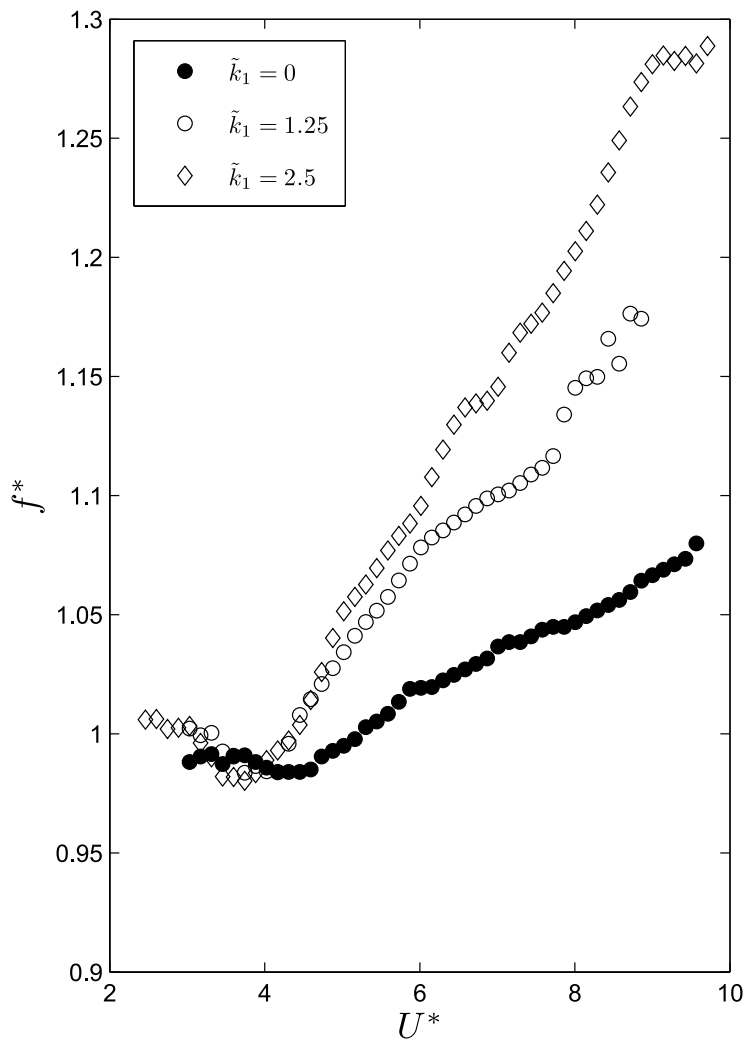

(b)

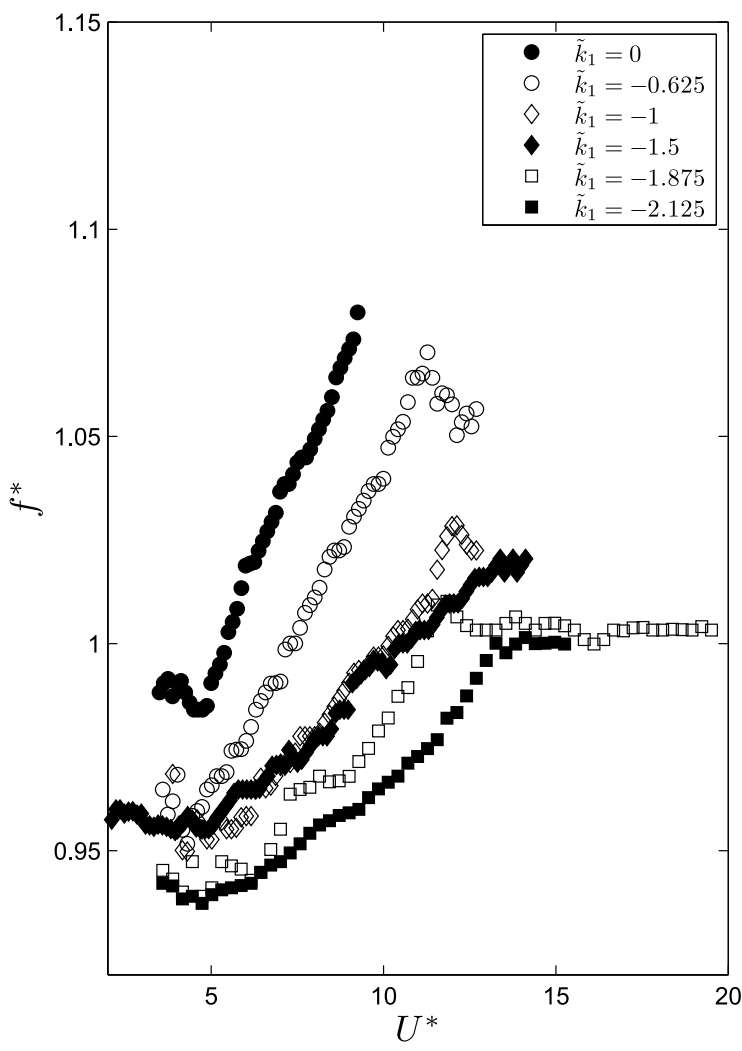

Figure 3.10: (a) Non-dimensional oscillating frequency of the cylinder as a function of the reduced velocity for positive values of the rotating parameter $\tilde{k}_{1}$ and (b) for negative values of $\tilde{k}_{1}$.

\subsubsection{PIV of the near wake for rotation proportional to the position}

In this subsection, Particle Image Velocimetry visualizations of the near wake are presented to understand the effect of the rotation of the prism on the wake patterns. Non-dimensional vorticity contours are given for different values of $\tilde{k}_{1}$ at two values of the reduced velocity, $U^{*}=5.2$ (near the peak amplitude) and $U^{*}=7.5$ which correspond to the upper and lower branches respectively in the classical VIV curve $\left(\tilde{k}_{1}=0\right)$. The non-dimensional vorticity contours images have been treated by POD (Proportional Orthogonal Decomposition) in order to retain high-level energy modes and to filter high spatial and temporal frequencies (for further detail the reader is referred to Ma et al. (2003) and Berkooz et al., 1993).

The classical VIV response, for the Reynolds numbers under consideration $(R e=3200)$ and for low enough mass-damping parameter $\left(m^{*} \zeta=0.0506\right)$, is characterized by a 3 branch curve. Regarding the wake patterns, the initial branch is characterized by a $2 \mathrm{~S}$ mode of vortex emission at the center line. The upper branch presents a $2 \mathrm{P}_{0}$ mode of vortex shedding structure (as described in Morse and Williamson, 2009) which consists in 2 pairs of vortices shed per cycle where the secondary vortex is smaller in intensity than the main one (Fig. 3.11a). In the lower branch, the secondary vortex has grown in intensity developing into a full $2 \mathrm{P}$ mode (Fig. 3.11b). Further increasing the velocity leads to a desynchronization between vortex emission and oscillation.

For $\tilde{k}_{1}>0$, the wake structure in the initial branch is equivalent to the case without actuation ( $2 \mathrm{~S}$ vortices shed near the center line). After the transition, near the peak amplitude, the secondary vortex is reduced and even dissapears as $\tilde{k}_{1}$ grows (Fig. $3.11 \mathrm{c}$ ), thus evolving from a $2 \mathrm{P}_{0}$ mode to a $2 \mathrm{~S}$ mode of vortex shedding (as $\tilde{k}_{1}$ is increased) where two single vortices are shed per cycle at the outer part of the oscillation's cycle (near its maximum amplitude). Also, the main vortex reduces 
its intensity compared to the case without actuation. This phenomenon probably occurs because the cylinder rotates in the opposite direction of the vortex being shed, therefore reducing the intensity of the circulation of the vortex shed which contributes to a reduction of the amplitude of oscillation. As will be seen in the next subsection, from a quasi-steady point of view, the lift coefficient depends on the rate of rotation $\dot{\theta}$ (Eq. 3.14); positive values of $\tilde{k}_{1}$ lead to a lift coefficient opposite to the velocity of displacement, thus contributing to a reduction of the amplitude of oscillation expected. Finally, as $U^{*}$ grows, the upper and lower branches are not distinguishable and desynchronization is anticipated (Fig. 3.11d).

For $\tilde{k}_{1}<0$, the transition between initial/upper branch is delayed, thus Figs. 3.11e and 3.11g, still yield a $2 \mathrm{~S}$ vortex emission mode at the center line as these contours still represent the wake structure of the initial branch. As $U^{*}$ increases, after the transition, a $2 \mathrm{P}$ mode is obtained (or $2 \mathrm{P}_{0}$ mode). The vortices shed increase in strength (compared to $\tilde{k}_{1}=0$ ) as $\tilde{k}_{1}$ becomes more negative, because the rotation of the cylinder is in the same direction as of the vortices being shed, thus increasing the circulation of such vortices (Figs. 3.11f and 3.11h) which contributes to the enhancement of the amplitude of oscillation. Noticeably, the vortices are shed at the outer part of the oscillation's cycle, thus, it seems that the rotation of the cylinder (for the range of $\tilde{k}_{1}$ investigated) does not modify the phase at which such vortical structures are emitted. The increase of amplitude is also related to the lift coefficient appearing due to the rotation of the cylinder (from a quasi-steady point of view) in the direction of the velocity of displacement contributing to the enhancement of the oscillations for negative values of $\tilde{k}_{1}$.

Furthermore, a case for $U^{*}=17$ and $\tilde{k}_{1}=-1.875$ is analyzed to observe the mechanism underlying oscillations for large negative values of $\tilde{k}_{1}$ and large $U^{*}$ in Fig. 3.12. Four different time snapshots are presented and overprinted in dashed line the past trajectory of the cylinder is given. As can be observed, the picture is very different from the previous ones where there was synchronization between vortex emission and oscillation of the prism. For this case, the vortices are shed independently from the cylinder's oscillation at its own frequency and thus the wake pattern resembles that of a wave as each vortex is shed independently at different points of the cycle of oscillation. In particular, the vortex emission is $f_{v}^{*}=3.44$ which compares reasonably well to that of the Strouhal value of vortex emission for a cylinder at rest $(\mathrm{St}=0.21)$ which yields $f_{S t}^{*}=3.57$. Thus, for large values of negative $\tilde{k}_{1}$ and reduced velocity, there is no synchronization between vortex emission and oscillation, and the amplitude response tends to a linear dependence with $U^{*}$ resembling a galloping-type of oscillation.

\subsubsection{Quasi-steady model for galloping-type response}

As observed in the previous subsection, there is a clear separation in the dynamical behavior between the cases for $\tilde{k}_{1}>-1.8$ and for those where $\tilde{k}_{1}<-1.8$. Summarizing, for $\tilde{k}_{1}>-1.8$, the dynamics of the cylinder is similar to a Vortex-Induced Vibration phenomenon where the frequency of oscillation is in synchronization with the frequency of vortex emission and thus the cylinder undergoes large oscillations. However, as the reduced velocity increases, the synchronization stops, and large amplitude oscillations disappear. $\tilde{k}_{1}$ only modifies the amplitude of oscillation as well as the intensity of the vortices shed, and enhances or diminishes the region of synchronization but does not modify the overall dynamical behavior of the cylinder. On the other hand, for large enough negative values of $\tilde{k}_{1}$, the amplitude of oscillation increases linearly with the reduced velocity leading to assume some kind of galloping-type phenomenon is happening. Also, the frequency of vortex emission for large $U^{*}$ follows the Strouhal law, therefore, increasing linearly with $U^{*}$ (as observed through PIV measurements), whereas the frequency of oscillation is locked at $f^{*} \approx 1$. A quasi-steady model is here proposed to determine for which values of $\tilde{k}_{1}$ a galloping-type behavior is expected to hold for large reduced velocity as well as a comparison between the amplitude of oscillation shed by the quasi-steady model and experimental results. 
$U^{*}=5.2$
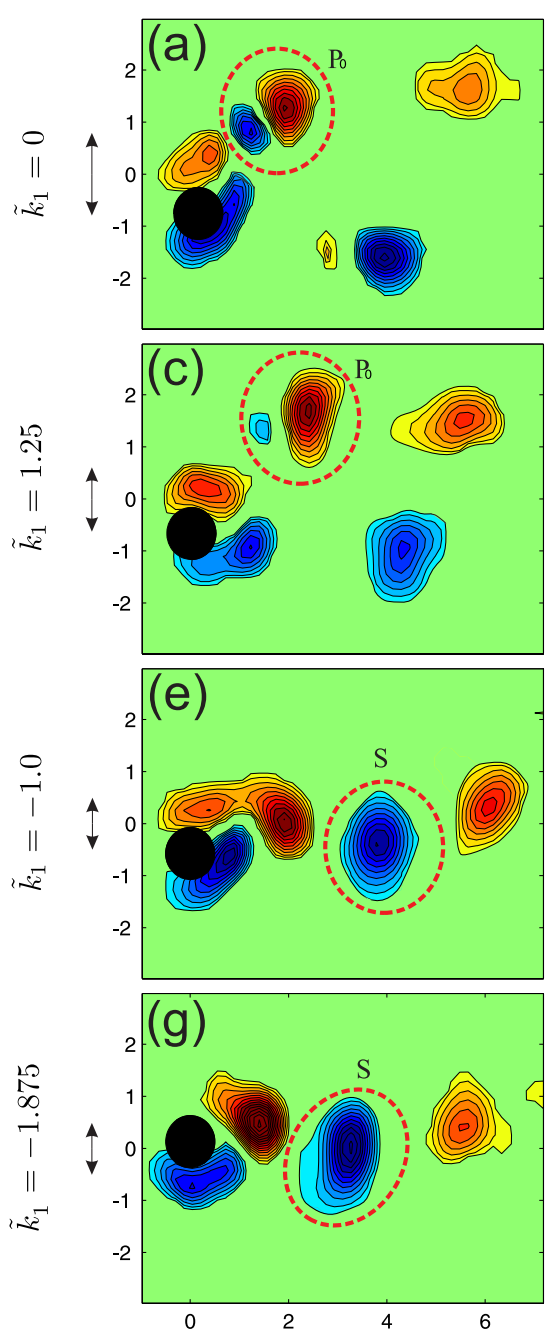

$U^{*}=7.5$
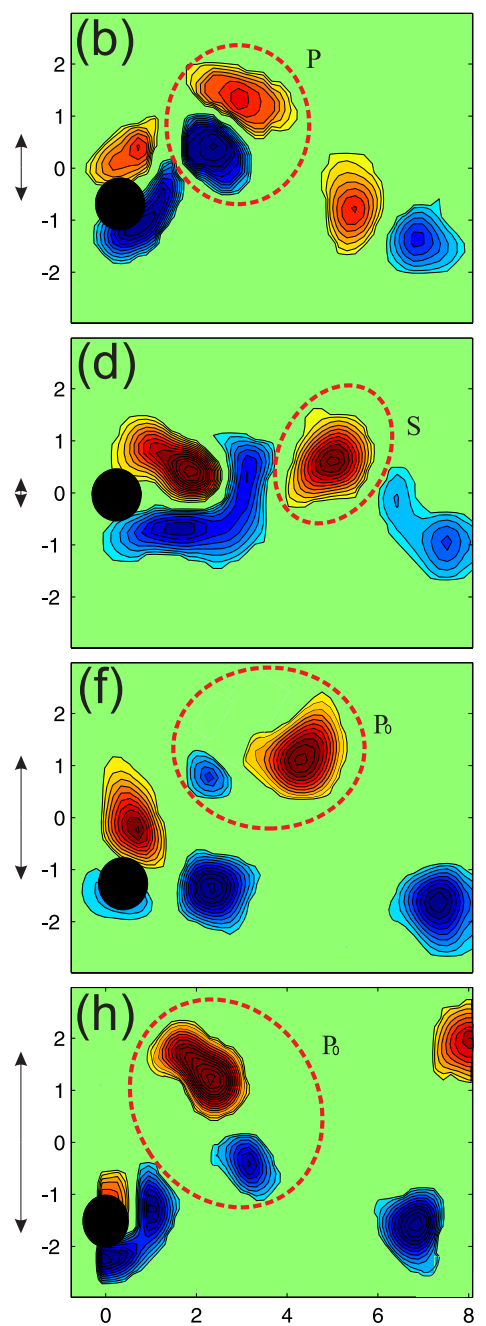

Figure 3.11: Non-dimensional vorticity contours $\omega D / U$ for two values of reduced velocity $\left(U^{*}=5.2\right.$, $7.5)$ and four values of $\tilde{k}_{1}\left(\tilde{k}_{1}=0,1.25,-1.0,-1.875\right)$ respectively. Red color indicates clockwise circulation and blue color indicates counter-clockwise circulation. Vortical structures are highlighted with dashed line. $\mathrm{P}$ represents a pair of vortices shed per half cycle corresponding to a $2 \mathrm{P}$ mode of vortex shedding. $\mathrm{P}_{0}$ is equivalent to the previous but with the secondary vortex being qualitatively smaller (circulation intensity wise). S structures represents a single vortex being shed per half cycle, which corresponds to a $2 \mathrm{~S}$ mode of vortex shedding. Note that for completeness, amplitude of oscillation for each case is indicated by an arrow line.

For the quasi-steady analysis, it is supposed that the transverse force at each instant can be obtained from the steady lift and drag force with an equivalent velocity with an angle of attack $\alpha_{F}$ (Blevins, 1990). In Fig. 3.13a a scheme of an equivalent configuration is given.

After projecting the lift and the drag forces, the transverse force coefficient can be obtained as:

$$
C_{y}=\frac{U_{r}}{U}\left(-C_{L} \cos \left(\alpha_{F}\right)-C_{D} \sin \left(\alpha_{F}\right)\right)=-\frac{C_{L}}{\cos \left(\alpha_{F}\right)}-C_{D} \frac{\tan \left(\alpha_{F}\right)}{\cos \left(\alpha_{F}\right)}
$$

where $\alpha_{F}$ is the instantaneous angle of attack, $\tan \left(\alpha_{F}\right)=-\dot{y} / U, \cos \left(\alpha_{F}\right)=U / U_{r}, U_{r}$ is the relative incident flow speed $\left(U_{r}=\sqrt{U+\dot{y}^{2}}\right)$ and $C_{L}$ and $C_{D}$ are, respectively, the lift and drag timeaveraged fluid force coefficients, which can be obtained from the 'static' configuration as a function of the rotation rate. Fig. $3.13 \mathrm{~b}$ shows the dependence of the lift coefficient with the rotation rate $(\alpha=\dot{\theta} D / 2 U)$ from experiments carried out in the water channel where the rotation rate was fixed 


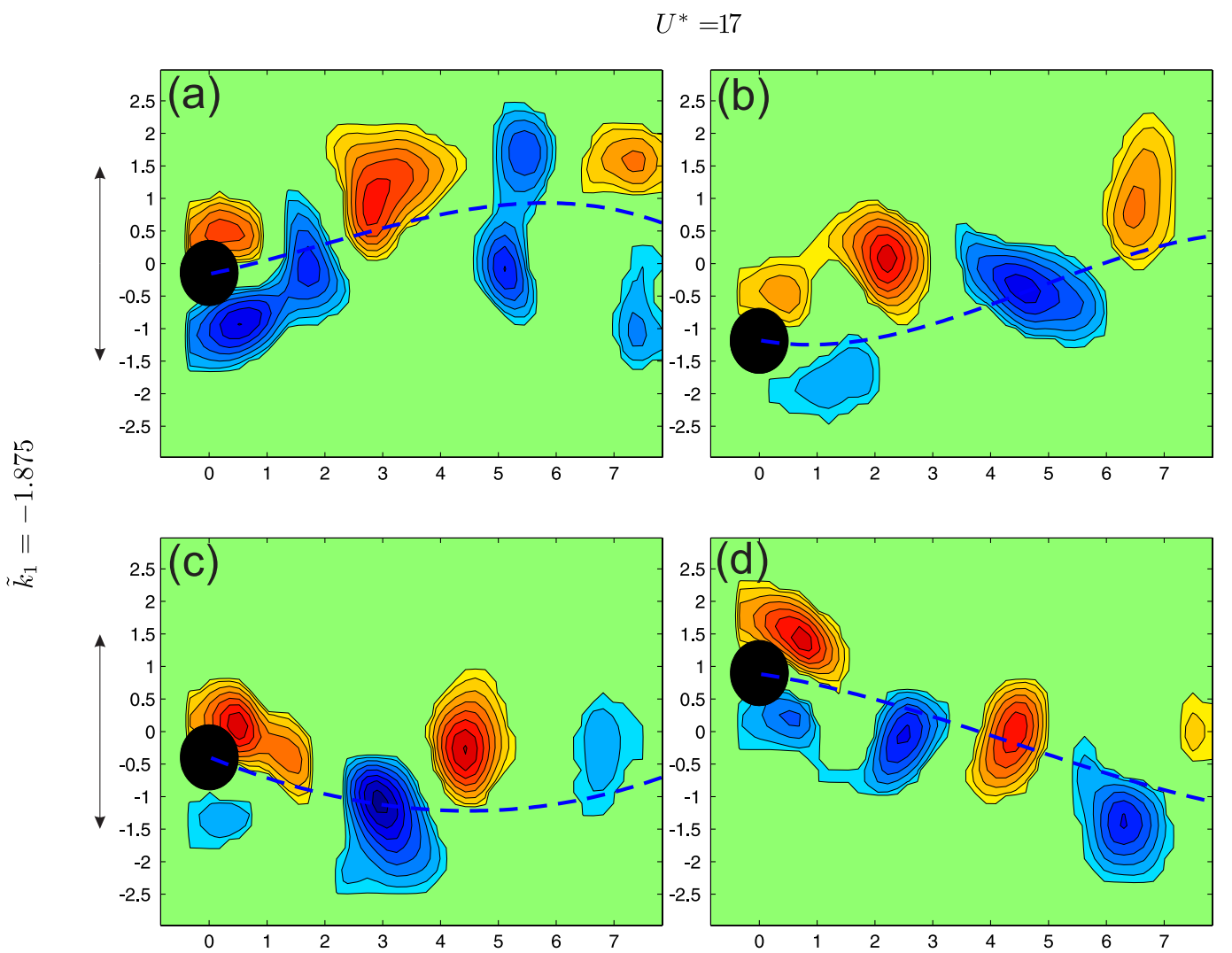

Figure 3.12: Non-dimensional vorticity $\omega D / U$ contours for $U^{*}=17$ and $\tilde{k}_{1}=-1.875$ evaluated at four different displacements of the cylinder. Red color indicates clockwise circulation and blue color indicates counter-clockwise circulation.

(a)

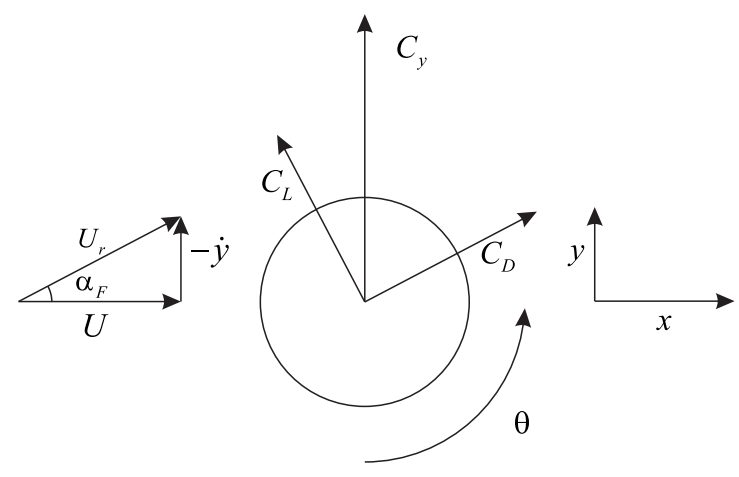

(b)

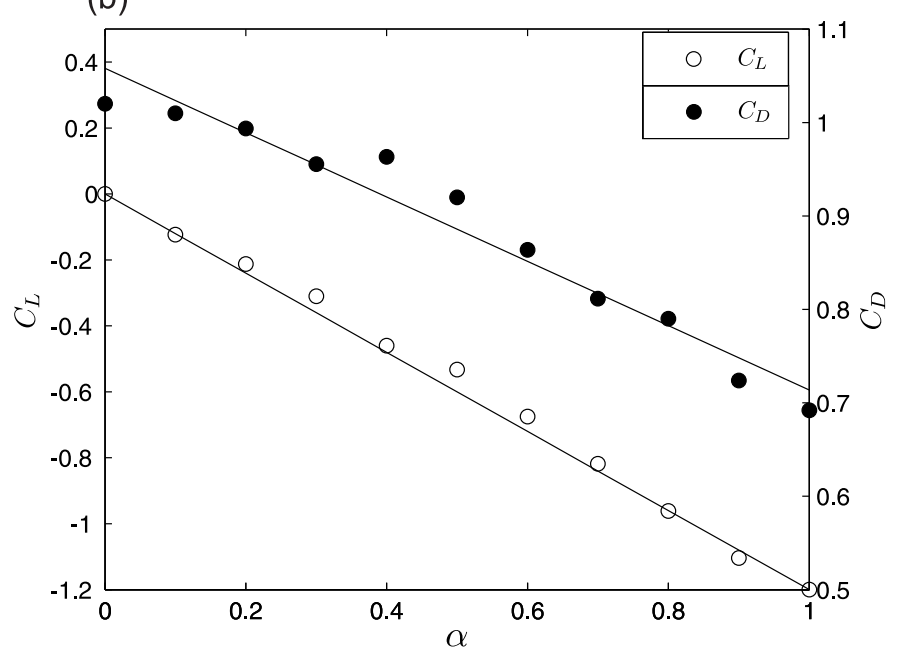

Figure 3.13: (a) Schematics of the forces acting on the cylinder in a quasi-steady situation. (b) Average transverse force coefficient and average in-line force coefficient as a function of the rotation rate $\alpha$. Experiments to determine $C_{L}$ and $C_{D}$ have been carried out at Re $\approx 9000$ which corresponds to $U^{*}=15$ for the current setup. Dashed lines represent the linear best fits.

at different values of $\alpha$ and fluid forces were measured using a force sensor. Assuming, as a first approximation, a linear variation with the rotation rate of $C_{L}$ and $C_{D}$ :

$$
C_{L}=C_{L \alpha} \alpha=C_{L \alpha} \frac{\dot{\theta} R}{U_{r}}
$$




$$
C_{D}=C_{D o}-C_{D \alpha}|\alpha|=C_{D o}-C_{D \alpha}\left|\frac{\dot{\theta} R}{U_{r}}\right|
$$

When the cylinder rotates proportionally to the cylinder's displacement $\left(\theta^{\prime}=\tilde{k}_{1} Y^{\prime}\right)$, they can be written in non-dimensional variables as

$$
\begin{gathered}
C_{L}=\frac{C_{L \alpha}}{2} \tilde{k}_{1} \frac{Y^{\prime}}{U_{r}^{*}}, \\
C_{D}=C_{D o}-\frac{C_{D \alpha}}{2}\left|\tilde{k}_{1} \frac{Y^{\prime}}{U_{r}^{*}}\right|,
\end{gathered}
$$

where $U_{r}^{*}=U_{r} / f_{N} D=U^{*} / \cos \left(\alpha_{F}\right)$ is the non-dimensional relative incident flow velocity. From Fig. 3.13b, the linear best fit yields $C_{L \alpha}=-1.2, C_{D o}=1.06, C_{D \alpha}=0.36$ which are in good correlation compared to similar experiments and numerical simulations found (Bourguet \& Lo Jacono (2014), Mittal \& Kumar (2003), Karabelas et al., 2012). Introducing Eqs. 3.13a and 3.13b into Eq. 3.11 one obtains

$$
C_{y}=-\frac{C_{L \alpha}}{2} \tilde{k}_{1} \frac{Y^{\prime}}{U^{*}}-C_{D o} \frac{Y^{\prime}}{U^{*}} \sqrt{1+\left(\frac{Y^{\prime}}{U^{*}}\right)}+\frac{C_{D \alpha}}{2}\left|\tilde{k}_{1} \frac{Y^{\prime}}{U^{*}}\right| \frac{Y^{\prime}}{U^{*}}
$$

which is the transverse force coefficient evaluated from a quasi-steady point of view. This model for the transverse force coefficient mainly neglects the effect of the vortices shed, which is valid for $U^{*}$ large enough with respect to $\mathrm{St}^{-1}$. The first term in Eq. 3.14 can be viewed as a linear damping for $\tilde{k}_{1}>0$ reducing possible amplitude oscillations or a linear negative damping term $\left(\tilde{k}_{1}<0\right)$ which on the other hand amplifies amplitude of oscillation. The last two terms are non-linear damping terms associated to the drag force. Note that there are no stiffness terms that could change significantly the frequency of oscillation thus recovering the expected frequency of oscillation which should coincide with $f_{N}$, that is $f^{*}=1$ (see Fig. $3.10 \mathrm{~b}$ for $\tilde{k}_{1}=-1.875, \tilde{k}_{1}=-2.125$ ).

It is of interest to study the value of $\tilde{k}_{1}$ from which the galloping-type response is expected to occur accordingly to the quasi-steady model. To do so, Eq. 3.14 can be expanded in Taylor series around $Y^{\prime} / U^{*}$ up to third order as

$$
C_{y}=-\frac{C_{L \alpha}}{2} \tilde{k}_{1} \frac{Y^{\prime}}{U^{*}}-C_{D o}\left(\frac{Y^{\prime}}{U^{*}}+\frac{1}{2}\left(\frac{Y^{\prime}}{U^{*}}\right)^{3}\right)+\frac{C_{D \alpha}}{2}\left|\tilde{k}_{1} \frac{Y^{\prime}}{U^{*}}\right| \frac{Y^{\prime}}{U^{*}} .
$$

Galloping-type oscillations will occur when the linearized term of the transverse force coefficients yields a positive value, thus

$$
\tilde{k}_{1}<-2 \frac{C_{D o}}{C_{L \alpha}} \simeq-1.76,
$$

which compares well with the results found during experimental results, since galloping-type oscillations appeared for $\tilde{k}_{1}<-1.8$. To obtain the amplitude of oscillations, the dynamical equation for the cylinder (Eq. 3.2) along with $C_{y}$ given by Eq. 3.14 can be solved analytically by the Harmonic Balance Method such as in Vicente-Ludlam $(2014,2015)$ or numerically with a Runge-Kutta scheme for example.

Fig. 3.14 assesses the validity of the quasi-steady model proposed with present experimental results for $\tilde{k}_{1}=-2.125$ with good agreement for high enough reduced velocity (say $U^{*}>8$ ) with errors bounded under $10 \%$. However, for $\tilde{k}_{1}=-1.875$ the quasi-steady model fails to predict the amplitude of oscillations of the experimental data as after an initial linear trend with $U^{*}$ the amplitude of oscillations keeps a nearly constant value until these cease at $U^{*}=20$. This could be caused by the low value of the linear part of the transverse force from Eq. 3.15, making other phenomena not taken into account in the quasi-steady model overcome galloping-type of oscillations. 


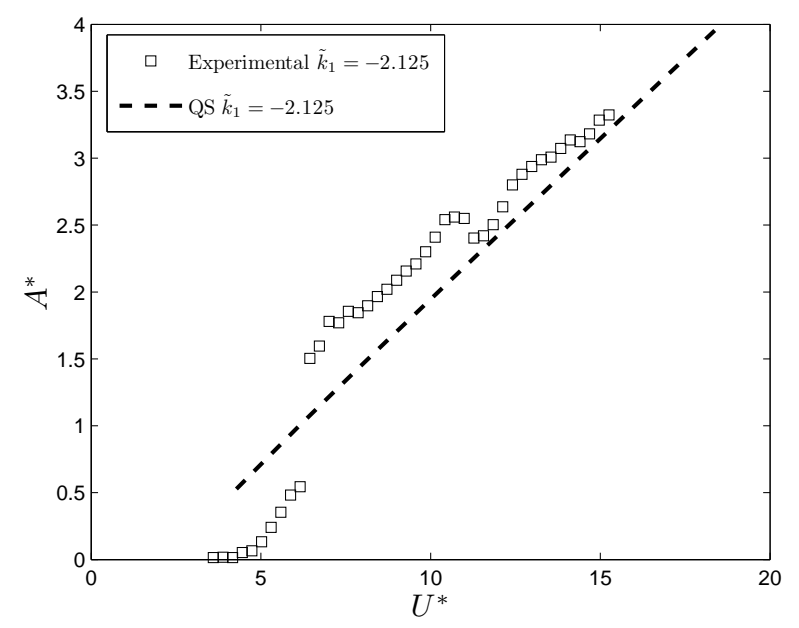

Figure 3.14: Comparison between quasi-steady solution and present experimental results for $\tilde{k}_{1}=$ -2.125 .

\subsubsection{Rotation proportional to velocity}

Another strategy proposed here is to modify the response of a circular cylinder undergoing VIV by rotating the cylinder proportionally to its own velocity. Results including amplitude and frequency of oscillation and non-dimensional vorticity contours are presented. In what follows, the cylinder is rotated according to the following feedback equation

$$
\theta=\tilde{k}_{2} Y^{\prime}
$$

where $\tilde{k}_{2}$ is a non-dimensional feedback constant.

\subsubsection{Cylinder dynamics}

In Fig. 3.15, the amplitude of oscillation of the cylinder is plotted as a function of the reduced velocity for different values of $\tilde{k}_{2}$. For $\tilde{k}_{2}>0$ VIV response is greatly attenuated with respect to the curve without actuation (Fig. 3.15a). With $\tilde{k}_{2}=1.25$, the peak amplitude is $A^{*} \approx 0.22$ whereas when rotating proportionally to the cylinder's displacement, for $\tilde{k}_{1}=2.5$ (which approximately leads to twice the level of rotation for a prescribed amplitude of oscillation) the maximum oscillation surpasses $A^{*}=0.5$. Thus rotating the cylinder proportionally to the velocity of displacement of the prism is considerably more efficient in terms of attenuating the amplitude of oscillation with less actuation. On the other hand, negative values of $\tilde{k}_{2}$ leads to an increase of the amplitude of oscillation as well as an increase of the synchronization region undergoing large amplitude oscillations. However, as can be seen in Fig. 3.15b, further decreasing $\tilde{k}_{2}$ does not modify the type of response obtained, namely, no galloping-type of oscillations have been found. This can be explained through Eq. 3.14 of the quasi-steady model, where if the first term is modified to include rotation proportional to the velocity:

$$
C_{y}=-\frac{C_{L \alpha}}{2} \tilde{k}_{2} \frac{Y^{\prime \prime}}{U^{*}}-C_{D o} \frac{Y^{\prime}}{U^{*}} \sqrt{1+\left(\frac{Y^{\prime}}{U^{*}}\right)}+\frac{C_{D \alpha}}{2}\left|\tilde{k}_{2} \frac{Y^{\prime \prime}}{U^{*}}\right| \frac{Y^{\prime}}{U^{*}},
$$

and no possible negative damping that can overcome the drag coefficient as well as the structural damping is present. Thus, the increase of the amplitude of oscillations has to be explained by the modification of the structure of the near wake (namely, intensity of vortices shed). The transition between the initial and upper branch is not as pronounced as for the case without actuation (or when rotating the cylinder proportionally to its position) and the reduced velocity at which the transition 

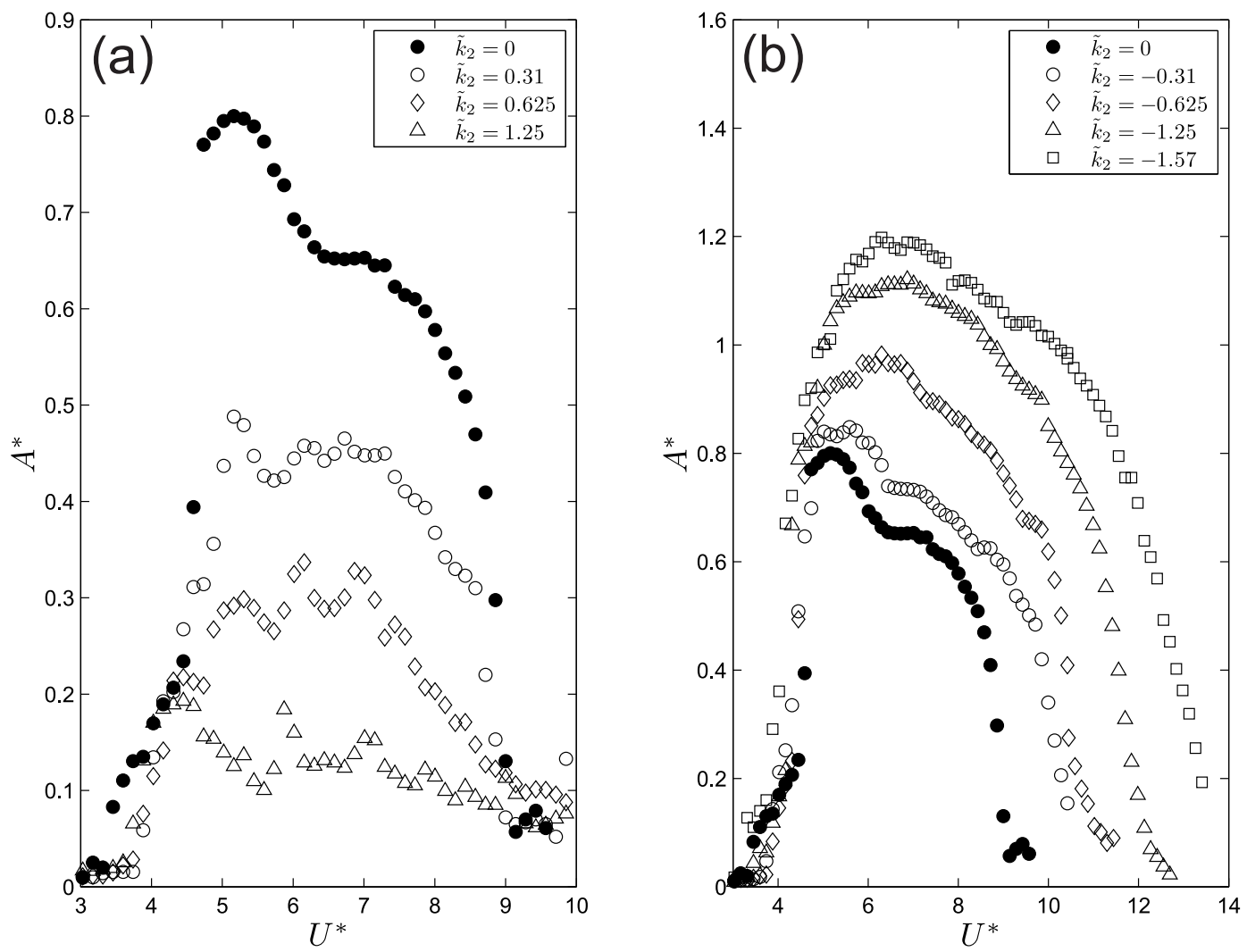

Figure 3.15: (a) Maximum non-dimensional amplitude oscillation as a function of the reduced velocity for positive values of the rotating parameter $\tilde{k}_{2}$ and (b) for negative values of $\tilde{k}_{2}$.

occurs is not modified by $\tilde{k}_{2}$. Also, the distinction between upper/lower branch is lost as actuation (either positive or negative) is increased.

Finally, with regard to the frequency of oscillation (Fig. 3.16), $\tilde{k}_{2}$ does not modify significantly $f^{*}$. The value of the non-dimensional frequency of oscillation nearly fits into a single curve for all values of $\tilde{k}_{2}$, thus explaining why the initial/upper branch transition occur at similar reduced velocity as the frequency of oscillation reaches $f^{*}=1$ at nearly the same reduced velocity. Negative values of $\tilde{k}_{2}$ (Fig. 3.16b) increases the region of synchronization, therefore, the frequency of oscillation keeps growing with the same slope until desynchronization occurs (for larger $U^{*}$ as $\tilde{k}_{2}$ becomes more negative).

\subsubsection{PIV of the near wake for rotation proportional to the velocity of displacement}

For positive values of $\tilde{k}_{2}$, the initial branch is not modified (presenting a $2 \mathrm{~S}$ mode of vortex shedding at the center line). At $U^{*}=5.5$ for $\tilde{k}_{2}=0$ (which corresponds to the upper branch), a $2 \mathrm{P}_{0}$ mode of vortex shedding was obtained (Fig. 3.17a). As $\tilde{k}_{2}$ increases, the amplitude of oscillation is diminished and still a $2 \mathrm{P}_{0}$ mode is observable (Fig. $3.17 \mathrm{c}, \tilde{k}_{2}=0.625$ ) though circulation intensity is reduced and vortices are shed nearer to the center line. Further raising $\tilde{k}_{2}$, leads to an increasingly nondistinctive wake pattern where $2 \mathrm{P}$ vortex emission is mixed with $2 \mathrm{~S}$ mode alternatively (driven by the low amplitude of oscillation as well as the rotation of the cylinder). As the reduced velocity is increased $\left(U^{*}=7.5\right)$, the picture is similar where $2 \mathrm{P}$ vortex emission (for $\tilde{k}_{2}=0$, Fig. $3.17 \mathrm{~b}$ ) slowly evolves to a $2 \mathrm{~S}$ mode as $\tilde{k}_{2}$ increases (Fig. $3.17 \mathrm{~d}$ exhibits a fully $2 \mathrm{~S}$ mode as for $\tilde{k}_{2}=0.625$ amplitude of oscillation is lower than 0.2 ).

For negative $\tilde{k}_{2}$, the initial branch is not modified (2S mode). After the transition, in the upper branch $\left(U^{*}=5.5\right)$, negative $\tilde{k}_{2}$ increases the circulation of the vortices shed very importantly compared 

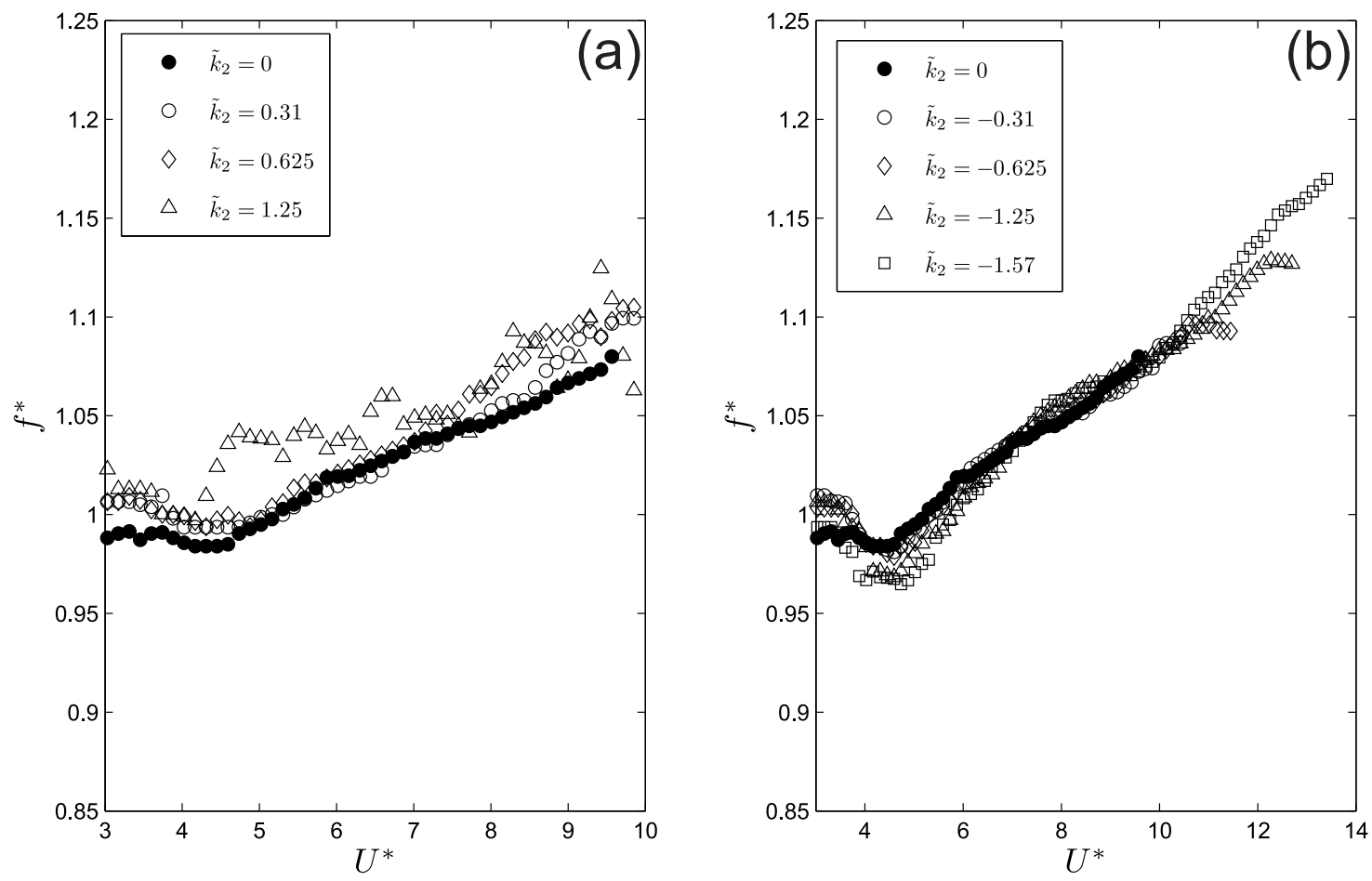

Figure 3.16: (a) Non-dimensional oscillating frequency of the cylinder as a function of the reduced velocity for positive values of the rotating parameter $\tilde{k}_{2}$ and (b) for negative values of $\tilde{k}_{2}$.

to the case without actuation and the secondary vortex is suppressed as $\tilde{k}_{2}$ is diminished (Figs. 3.17e and $3.17 \mathrm{~g}$ ). For $U^{*}=7.5$ a $2 \mathrm{P}$ mode of vortex emission (similar to $\tilde{k}_{2}=0$ ) is obtained with increasing vortex circulation intensity as $\tilde{k}_{2}$ is diminished with also a diminishing intensity of the secondary vortex (Fig. $3.17 \mathrm{f}$ and $3.17 \mathrm{~h}$ ). As observed, rotating the cylinder proportionally to its velocity mainly affects the intensity of the vortices shed for this range of $U^{*}$ without evidently affecting the phase of vortex emission.

Notably, as seen in Fig. 3.15b, synchronization region can be enlarged with negative $\tilde{k}_{2}$. In particular, non-dimensional vorticity contour maps are given at $U^{*}=9.5$ for the case without actuation $\left(\tilde{k}_{2}=0\right.$, Fig. 3.18a) and two negative values of $\tilde{k}_{2}\left(\tilde{k}_{2}=-0.625, \tilde{k}_{2}=-1.25\right)$, Figs. 3.18b and $3.18 \mathrm{c}$ respectively). As observed, for $\tilde{k}_{2}=0$, oscillations are small due to desynchronization between vortex emission and the cylinder's oscillation. However, as $\tilde{k}_{2}$ becomes more negative, the synchronization region is enlarged and large amplitude oscillations occur and a fully developed $2 \mathrm{P}$ mode can be observed. Finally, no quasi-steady flow pattern has been found with regard to the wake pattern (as depicted from Eq. 3.18).

\subsection{Concluding remarks}

Hydroelastic experiments have been performed to study the dynamical behavior and wake pattern of the fluid flow around a circular cylinder undergoing FIV (for $m^{*}=11.7$ and Reynolds number 3200 at the peak of the VIV curve response), free to oscillate in the transverse direction with an imposed rotation around its axis linked to the cylinder's dynamics. Two different rotating laws have been investigated in particular, first a rotation proportional to the cylinder's position and second a rotation was given proportional to the cylinder's velocity. The main findings are:

- Regarding the case where rotation is proportional to the cylinder's displacement $\left(\theta=\tilde{k}_{1} Y\right)$ it has been found that positive values of $\tilde{k}_{1}$ lead to a reduction in the maximum amplitude of oscillation 
compared to that of the non-rotating VIV, and the transition between the initial-to-lower branch happened for lower values of reduced velocities. Also, the frequency of oscillation was slightly increased and the region of VIV synchronization was diminished. On the other hand, negative values of $\tilde{k}_{1}$ increases importantly the maximum peak value of oscillations as well as it increases the reduced velocity at which such maximum occurs. Also, the region of synchronization is greatly enlarged as $\tilde{k}_{1}$ becomes more negative. In fact, if $\tilde{k}_{1}$ overcomes a certain negative value, the response obtained changes dramatically and a galloping-type of response is obtained, where the amplitude of oscillation increases almost linearly for high values of the reduced velocity. In those cases, the frequency of oscillation remains constant ( $f^{*}$ close to 1$)$ for high values of reduced velocity and the frequency of vortex shedding increases approximately following the Strouhal law and thus both timescales depart from each other. A large number (increasing with the reduced velocity) of vortices are shed per oscillation cycle. From the practical side, note that negative values of $\tilde{k}_{1}$ could be of interest for energy harvesting purposes as well as to promote mixing.

- A quasi-steady model has been proposed in order to predict the amplitude of oscillation for large negative values of $\tilde{k}_{1}$. When the timescales of the vortex emission and the oscillation period are importantly separated, the force can be considered in a quasi-steady fashion and the $C_{y}$ force coefficient can be expressed as a function of $Y^{\prime} / U^{*}$. Following Eq. 3.16, galloping condition is fulfilled for negative values larger than $\tilde{k}_{1}<-2 C_{D o} / C_{L \alpha}=-1.76$. A linear dependence of the amplitude of oscillation with the reduced velocity is obtained. As $\tilde{k}_{1}$ becomes more negative, the slope of the amplitude oscillation with the reduced velocity increases. The quasi-steady model was compared to experimental results with good agreement between them.

- For the case where rotation is proportional to the cylinder's velocity $\left(\theta=\tilde{k}_{2} Y^{\prime}\right)$ it has been found that positive values of $\tilde{k}_{2}$ lead to a reduction in the maximum amplitude of oscillation compared to that of the pure VIV. Notably, this rotation law leads to greater reduction of the amplitude of oscillation of the prism with less actuation compared to rotating the cylinder proportionally to its displacement (with $\tilde{k}_{1}>0$ ). On the other hand, negative values of $\tilde{k}_{2}$ increases the maximum value of oscillation. Regarding the frequency of oscillation, it nearly fits into a curve for all values of $\tilde{k}_{2}$. Negative values of $\tilde{k}_{2}$ increases the region of synchronization, thus $f^{*}$ keeps growing with $U^{*}$ until desynchronization occurs and the vortex shedding frequency is always synchronized with the frequency of oscillation. For all values of $\tilde{k}_{2}$, oscillations occur under vortex induced vibrations (bell-shaped evolution as a function of the reduced velocity).

- The quasi-steady model developed helps to explain why galloping-type of response is not expected to occur for the case of rotating the cylinder proportional to its velocity as the transverse force term associated to the rotation is proportional to the acceleration, thus there is no excitation that overcomes the drag coefficient. This agrees with experimental results and PIV visualizations performed in which all large-amplitude oscillations obtained occur under VIVtype of solution.

- In general, wake modes found for the parameter set of $U^{*}, \tilde{k}_{1}$ and $\tilde{k}_{2}$ investigated are equivalent to those observed for the non-rotating case (Williamson and Roshko (1988), Williamson and Govardhan (2004)), that is, modes $2 \mathrm{~S}, 2 \mathrm{P}_{0}$ and $2 \mathrm{P}$. Rotation of the prism mainly modifies the intensity of the circulation of the vortices shed. Positive values of $\tilde{k}_{1}$ and $\tilde{k}_{2}$ lead to a reduction in their intensity of circulation whereas negative values of $\tilde{k}_{1}$ and $\tilde{k}_{2}$ increase such intensity as a general trend. This is mainly caused by the direction of rotation of the cylinder as well as its rotation rate. However, for high enough negative values of $\tilde{k}_{1}$ and high $U^{*}$, a new wake mode of vortex shedding has been found where the vortex formation is decoupled from the oscillation of the cylinder. The vortices are shed independently from the cylinder's oscillation at its own 
frequency and thus the wake pattern resembles that of a wave as each vortex is shed at different points of the cycle of oscillation. Further experiments for higher values of the feedback constants $\tilde{k}_{1}$ and $\tilde{k}_{2}$ should be performed to determine if new modes of vortex emission are expected. 

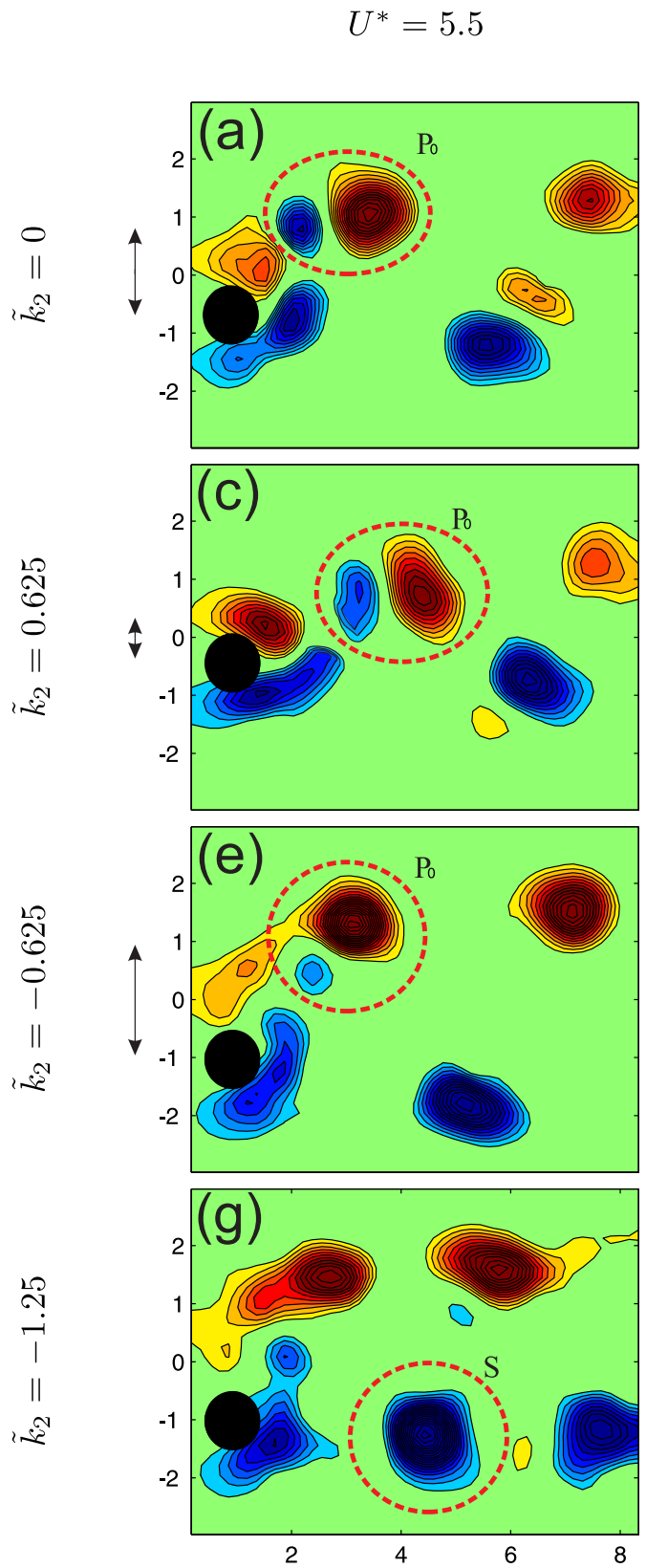
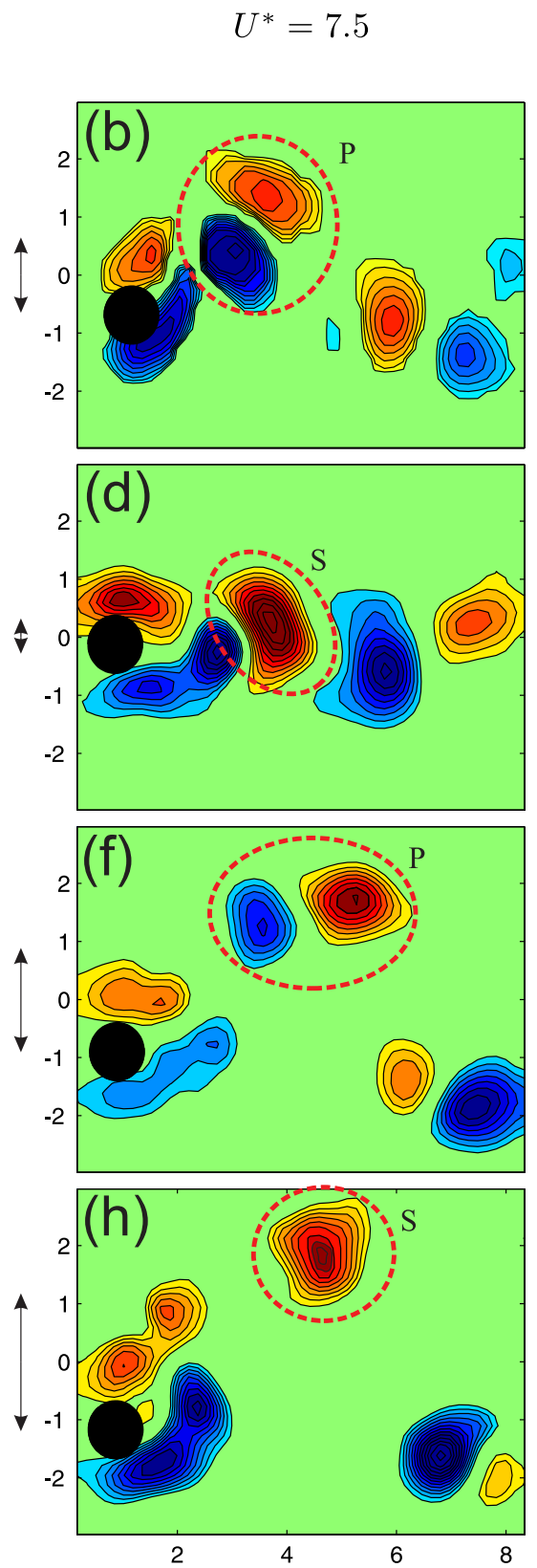

Figure 3.17: Non-dimensional vorticity contours for two values of reduced velocity $\left(U^{*}=5.5,7.5\right)$ and four values of $\tilde{k}_{2}\left(\tilde{k}_{2}=0,0.625,-0.625,-1.25\right)$ respectively. Red color indicates clockwise circulation and blue color indicates counter-clockwise circulation. Vortical structures are highlighted with dashed line. P represents a pair of vortices shed per half cycle corresponding to a $2 \mathrm{P}$ mode of vortex shedding. $\mathrm{P}_{0}$ is equivalent to the previous but with the secondary vortex being qualitatively smaller (circulation intensity wise). S structures represents a single vortex being shed per half cycle, which corresponds to a $2 \mathrm{~S}$ mode of vortex shedding. Note that for completeness, amplitude of oscillation for each case is indicated by an arrow line. 


$$
U^{*}=9.5
$$
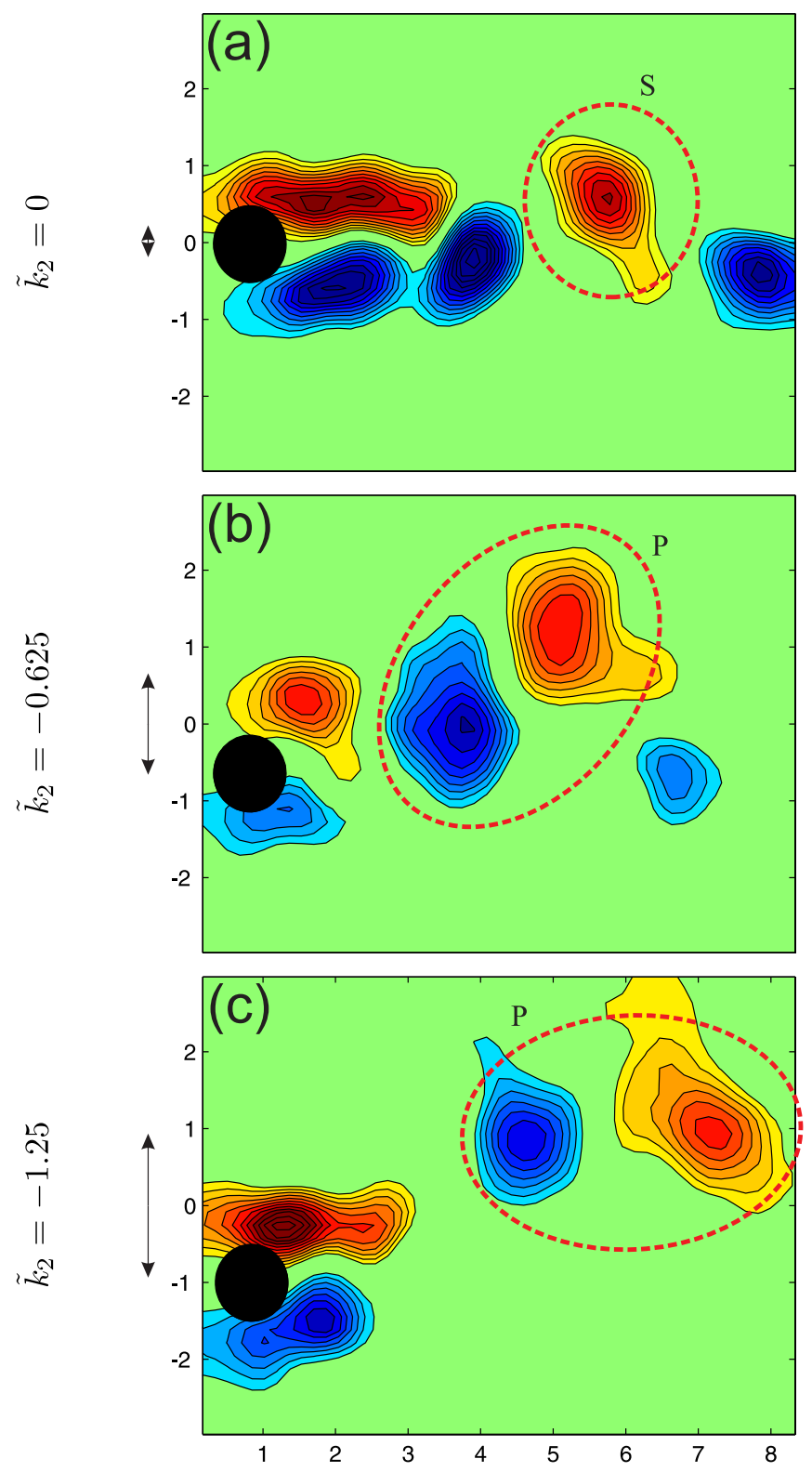

Figure 3.18: Non-dimensional vorticity contours for $U^{*}=9.5$ and three values of $\tilde{k}_{2}\left(\tilde{k}_{2}=0\right.$, $-0.625,-1.25)$ respectively. Red color indicates clockwise circulation and blue color indicates counterclockwise circulation. Vortical structures are highlighted with dashed line. $\mathrm{P}$ represents a pair of vortices shed per half cycle corresponding to a $2 \mathrm{P}$ mode of vortex shedding. $\mathrm{P}_{0}$ is equivalent to the previous but with the secondary vortex being qualitatively smaller (circulation intensity wise). S structures represents a single vortex being shed per half cycle, which corresponds to a $2 \mathrm{~S}$ mode of vortex shedding. Note that for completeness, amplitude of oscillation for each case is indicated by an arrow line. 


\section{Chapter 4}

\section{Optimal energy harvesting from transverse galloping}

\subsection{Introduction}

\subsubsection{Brief introduction of the galloping phenomenon}

Transverse galloping is a fluid-elastic instability of one degree of freedom that appears in some elastic bluff bodies when the velocity of the incident flow exceeds a critical value. Then, a small transverse displacement of the body induces an angle of attack relative to the incoming flow and a fluid force appears in the direction of the displacement in such a way that energy is pumped from the current to the body. Oscillatory motion (transverse to the flow) develops with increasing amplitude until the energy dissipated per cycle by mechanical damping balances the energy input per cycle from the flow. It was first described by Lanchester (1907) where the galloping of a D-section body with its flat surface facing the flow was treated. It is characterized by being an autoexcited, low-frequency phenomenon, with large amplitude oscillations transverse to the incident flow where only some specific cross-sections of the body are prone to galloping. These galloping-induced vibrations are usually considered to be an undesirable effect for civil or marine structures as they can reduce their lifetime by fatigue loading, compromise their service, or put at risk their integrity.

For example, these can arise in electric cables situated in cold areas where it snows frequently, making it possible for the snow to stick to the cables and bringing out aerodynamic shapes that favor galloping (Rawlins, 1979). It may also appear in marine conduction where organic matter deposit and produce an elliptical shape (Simpson, 1972). Light structures with specific cross-sections (namely, rectangular, triangular, D-sections or H-shaped) may be prone to suffer galloping, e.g. traffic signs or bridge decks of certain cross-sectional shapes, though the mechanism for this case may be more complex (Naudascher 1974). For an authoritative introduction to transverse galloping phenomenology and modeling, the reader is referred to Parkinson (1989), or the books by Blevins (1990), Naudascher and Rockwell (1994), or Paidoussis et al. (2012).

The key element to determine if a structure is susceptible to suffer transverse galloping oscillations is namely its cross-section geometry. Only cross-sections in which a transverse displacement $y$ (which induces an angle of attack $\alpha_{F}$ with respect to the fluid flow) produces a transverse force $F_{y}$ in the direction of the displacement are prone to galloping. Fig. 4.1a shows the fluid flow of a cross-section (in this case a D-section) in which as the induced angle of attack $\alpha_{F}$ increases, the current in the bottom part is attached inducing a pressure drop, whereas in the top part, the flow is fully detached thus increasing the pressure. This difference in pressure between both sides, induces a transverse force $F_{y}$ in the direction of the movement, therefore energy is transferred from the flow to the structure 
and oscillations may appear if the structural parameters are correctly matched.

(a)
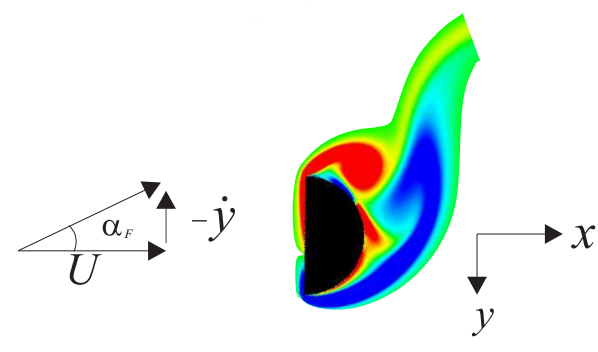

(b)

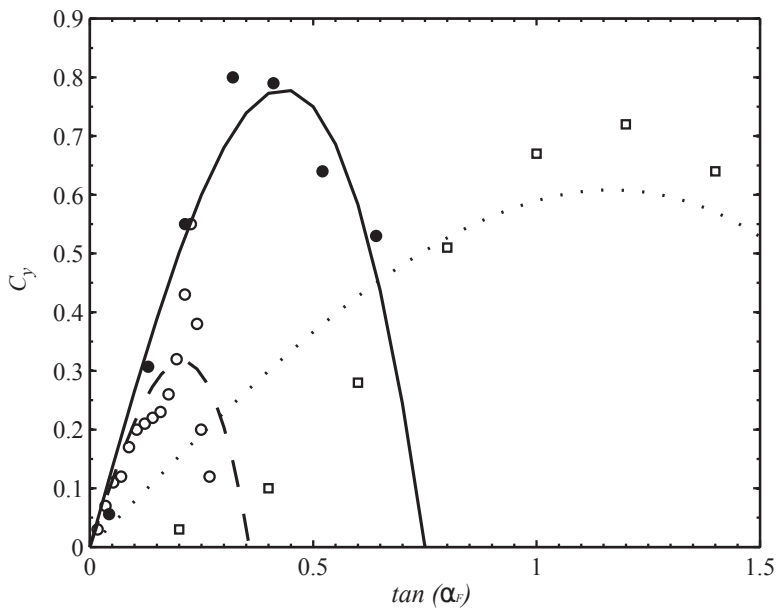

Figure 4.1: (a) Vorticity contour around a D-section at $\mathrm{Re}=300$ with an equivalent positive angle of attack obtained through LBM method. Observe that the flow is attached in the lower part of the body inducing a lower pressure region whereas in the top part the flow if fully detached. Then, a fluid force appears in the direction of the motion thus energy is being pumped into the galloping body from the flow. (b) Steady transverse force coefficient for a square (white circles), isosceles triangle (black circles) and D-section (open squares) cross-sections. Lines correspond to the fitting curves.

Complexity level of the analysis of transverse galloping vary widely determined by the results expected. These can range from experimental results conducted in wind tunnels or water channels, numerical simulations or down to linearized models. In this chapter, a non-linear quasi-steady model is resorted to to describe the transverse forces appearing on the structure. Quasi-steady fluid dynamics are applicable if the characteristic timescale of motion $t_{M}=1 / f_{N}{ }^{1}$ is much larger than the characteristic convective timescale $t_{R}=D / U^{2}$. Quantifying properly this criterion concerning the applicability of the quasi-steady fluid dynamics is still an open question. For example, according to Blevins (1977), the criterion for quasi-steady fluid dynamics is that the frequency of vortex shedding (Strouhal frequency $\left.f_{S t}=\operatorname{St} U / D\right)$ must be at least twice as large as the oscillation frequency $f_{N}$. Assuming a Strouhal number $\mathrm{St}=0.2$, the criterion to be satisfied is

$$
\frac{U}{f_{N} D}>10
$$

However, this is only indicative, as a matter of fact, Blevins (1990) revised the criterion up to $U / f_{N} D>$ 20. If quasi-steady hypothesis is fulfilled, the instant fluid forces appearing on the structures can be considered to be equivalent to the static fluid force force evaluated at the instantaneous angle of attack. Predictions obtained from quasi-steady analysis are satisfactory as long as the hypothesis hold. However, experiments in water channels (Nemes et al. (2012), Zhao et al. (2014), or Xu-Xu et al., 2016a) suggest that the quasi-steady model is unable to predict some features for density ratios (between the galloping body and the fluid under consideration) of the same order: mainly, (i) frequency of oscillation may significantly depart from its natural frequency and (ii) phase lag between fluid force and oscillations is not $90^{\circ}$ as quasi-steady hypothesis predicts. Luo and Bearman (1900), through an extensive experimental campaign carried by means of forced vibrations for a square prism suggested that the quasi-steady conditions where not related with the reduced velocity $\left(U^{*}=U / 2 \pi f_{N} D\right)$ but

\footnotetext{
${ }^{1} f_{N}$ is the natural frequency of oscillation.

${ }^{2} D$ corresponds to a characteristic length of the cross-section and $U$ to the undisturbed velocity of the incident cross-flow
} 
rather with the parameter $U^{*} / A^{*}$ (reduced velocity divided by the non-dimensional amplitude of oscillation), suggesting that quasi-steady conditions only occur for large values of $U^{*} / A^{*}$. A recent study in our investigation group (Ji Xu Xu Thesis) has proposed a quasi-steady time-delayed model capable of reproducing the most notable features for unsteady regime of transverse galloping and also explaining why previous criteria for quasi-steady conditions were so discordant. Nevertheless, for the ongoing chapter, it will be considered that the quasi-steady model is sufficient to collect the required dynamical behavior for the transverse galloping phenomenon.

Back to the quasi-steady model, Den Hartog (1930) first established the critical velocity where galloping oscillations appear through linearized quasi-steady aerodynamic forces. However, in order to describe the time evolution of the movement of the structure once transverse galloping appears it is necessary to retain non-linear terms of the aerodynamic force. Such terms limit the amplitude of oscillations and Limit Cycle Oscillations (LCO) are achieved, where the energy pumped into the structure from the incoming flow equals the energy dissipated. This non-linear analysis was first described by Parkinson (1964), where a 1 degree of freedom structure with non-linear transverse forces (described through the quasi-steady hypothesis) acting on the structure were considered. Through his analysis, the Limit Cycle of Oscillations as a function of the incoming fluid flow was obtained. Interestingly, hysteresis phenomena that had been reported in experimental tests could be addressed through this non-linear analysis.

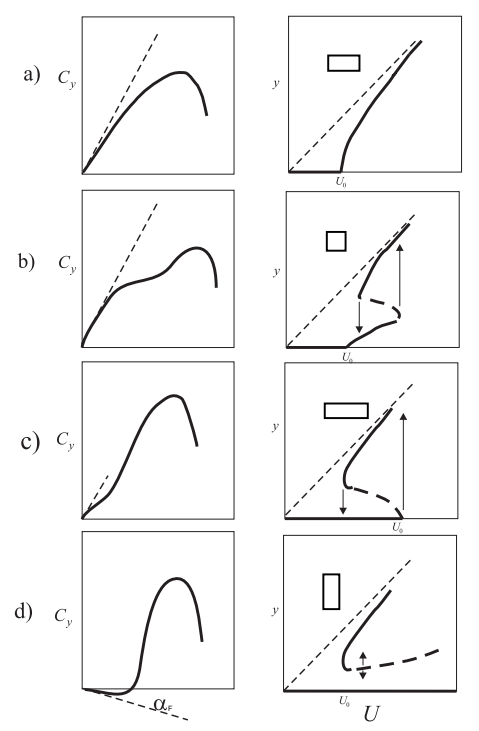

Figure 4.2: Steady transverse force coefficients $C_{y}$ for different rectangular cross-sections with the corresponding four main characteristic amplitude responses.

Novack (1969) investigated the galloping response of different cross-sections, showing that four basic types of amplitude of oscillation response were expected as a function of the curve of the transverse force coefficient $C_{y}=2 F_{y} / \rho U^{2} D$ with respect to the angle of attack $\alpha_{F}$ (see Fig. 4.2). For curves type a) and b), the slope of $C_{y}$ at zero angle of attack is positive and the instability is associated with a supercritical Hopf Bifurcation at $U=U_{0}$, after which the equilibrium position is no longer stable (soft oscillator). Also, b) curve presents hysteresis phenomena for the amplitude of oscillation in a range of flow velocities (with two stable and one unstable branches). For curve c), a subcritical Hopf Bifurcation appears (hard oscillator) where oscillations appear for infinitely small perturbations after the critical velocity $U_{0}$ is overcome. Before $U_{0}$ there is a range of reduced velocities where oscillations are possible if initial perturbation reaches a determined value, thus, there are two stable branches (being one of them the equilibrium position) and an unstable branch in between. For the d) curve, the slope of the transverse force coefficient is negative, (stable under small perturbations) and only 
oscillations are possible if there is a high enough initial perturbation. After the critical velocity $U_{0}$ is overcome, there are two stable branches and one unstable branch in between, being the equilibrium position always stable for all values of the reduced velocity (hard oscillator). With respect to the hysteresis, Barrero-Gil et al. (2009) investigated the hysteresis phenomena appearing on transverse galloping. They unequivocally showed the link between the hysteresis phenomenon (and the number of LCO appearing) and the number of inflection points appearing on $C_{y}$.

Even though $C_{y}$ is mainly affected by the cross-section of the structure and the angle of attack, many other parameters or phenomena may importantly modify $C_{y}$. To name a few: turbulence intensity, three-dimensional effects, roughness of the body's wall and to some extent to the Reynolds number.

\subsubsection{Galloping as a mechanism for energy harvesting}

The idea of using transverse galloping as a way of extracting energy from a flow was first investigated by Barrero-Gil et al. (2010). They employed a quasi-steady approximation to model the fluid forces and found an analytical expression for the energy transferred from the flow to the galloping body as a function of the mass ratio, the mechanical damping, the reduced velocity, and the geometry of the body. If the geometry of the body and the elastic properties are appropriate, the instability may appear at low flow velocities and with large excitation amplitudes, making transverse galloping a very promising way to harvest energy successfully. Furthermore, transverse galloping is an instability phenomenon as opposed to other resonant phenomenons (such as VIV), where large amplitude oscillations are expected to happen only in a range of velocities of the incident flow. Transverse galloping potential for energy harvesting was further investigated theoretically, numerically, and experimentally by Abdelkafi and co-workers (2012a,2013a), Sirohi and Mahadik (2011), or Zhao et al. (2012).

In a simplified manner, the energy-focused galloping system is comprised of three main elements: (i) a transducer, where the power extracted from the flow is converted into electrical energy, (ii) the elastic system provides the restoring force and allows oscillations to happen and (iii) the galloping body immersed in the fluid flow where the transverse fluid forces appear responsible for the galloping phenomenon. The problem is fully coupled (double coupling), thus an integral perspective has to be taken into account in order to fully establish design rules to be able to harness energy from the current in an efficient manner. In this chapter, different strategies to increase the efficiency of the energy extraction from cross-flows using the transverse galloping phenomenon are investigated, with a focus on each of the three mentioned elements:

1. Transducer: In first place, the effect of introducing a transducer to produce electrical current is investigated. In particular, two types of transducers are considered, an electromagnetic and a piezoelectric one. Optimization of the different parameters involved in the problem is performed to maximize the efficiency of energy extraction (and minimizing losses in the process of energy conversion).

2. Elastic system: The possibility of modifying the elastic system to increase the efficiency of energy extraction is investigated, and a dual mass system is proposed. In this case, a secondary mass is elastically mounted to the main galloping body in three different mechanical configurations comparing performances regarding maximum efficiency.

3. Galloping body: The effect of actively orientating the galloping body is studied and different rotation laws are explored so as to determine the possible increase in the maximum efficiency of energy extraction. Basically, it is sought to rotate the galloping body in such a way that $C_{y}$ is maximized during all the cycle of oscillation. Thus, the energy pumped from the flow to the galloping structure is significantly increased. Inspiration came from the swimming capabilities of fish where the opposite strategy is used. At each instant the fins of the fish are actively 
positioned in order to maximize energy transfer, but in this case from the body to the fluid in order to propel itself with minimum use of energy.

Summarizing, the following questions have guided the work presented and developed in this chapter

1. How does a transducer affect galloping phenomenon? Are differences in dynamical behavior expected for distinct transducers? In particular, which transducer is better efficient-wise?

2. Can different elastic systems be considered? Is it more efficient to use a dual mass system? Would it be interesting to even use more elastically-mounted masses?

3. How does it affect to orientate actively the galloping body? Can more power be harnessed just by modifying the angle of attack at any given instant? Can the frequency of oscillation be modified?

The chapter is organized as follows, in Section 4.2 the mathematical model is considered where the dynamical effect of a generic transducer (for electricity generation) is included. In Section 4.3, an electromagnetic generator is considered. A mathematical model of the transducer is coupled with the galloping model and energy extraction is analyzed. Guidelines on how to tune the electromagnetic generator as well as a practical example are proposed. In Section 4.4 a piezoelectric transducer model is developed and coupled to the galloping body. A study on how to correctly design the piezoelectric sheets' properties is discussed and a validation of the method proposed is compared to experiments from Zhao et al. (2013). In Section 4.5 a dual mass elastic system is considered with 3 different elastic configurations and comparison on the maximum efficiency that can be harvested is shown. In Section 4.6 the effect of externally rotating the galloping body is studied, evaluating the effect it has on the transverse force coefficient $C_{y}$ and in the efficiency. Static $C_{L}$ and $C_{D}$ curves needed for the quasi-steady model as a function of the angle of attack $\alpha_{F}$ for a D-section have been obtained through a LBM numerical computation at Reynolds 100. Dynamical response is compared between the results of the quasi-steady model and LBM computations at $R e=100$. 


\subsection{Formulation of the problem}

\subsubsection{Mathematical model}

In general, any three-dimensional structure considered immersed in an incident flow current is subject to fluid dynamic forces which are very complex and costly to model, numerically and analytically speaking. However, it is possible to simplify the model as far as to become approachable without losing relevant information about the dynamical response being sought. In particular for the case in question (Fig. 4.3), the system comprises a slender prism $(L / D \gg 1)$ with constant cross-section, elastically-mounted and free to oscillate in the transverse direction and subject to fluid forces that can be modeled through the quasi-steady hypothesis. Furthermore, if the elastic structure is considered to behave linearly, it is possible to describe its dynamical behavior through a modal analysis (and be modeled through a set of ordinary differential equations). In this chapter, as above mentioned the structure is restricted to oscillate transverse to the incident current $y$, hence a 1 degree-of-freedom is examined.

With the previous considerations in mind, let us consider a simplified dynamical system which consists of a spring-mounted prismatic body, prone to galloping, in an incoming flow. The assembly can be better understood in Fig. 4.3. As it can be seen, the system is composed of a viscous parasite damper, with constant damping per unit length $c$, a linear spring, with constant stiffness per unit length $k$, and with the purpose of electrical energy generation, a transducer used as energy harvester which induces a generic backlash force $F_{T}$. The prism has a mass per unit length of $m$ and is restricted to move in the $y$ direction (transverse to the incident flow).
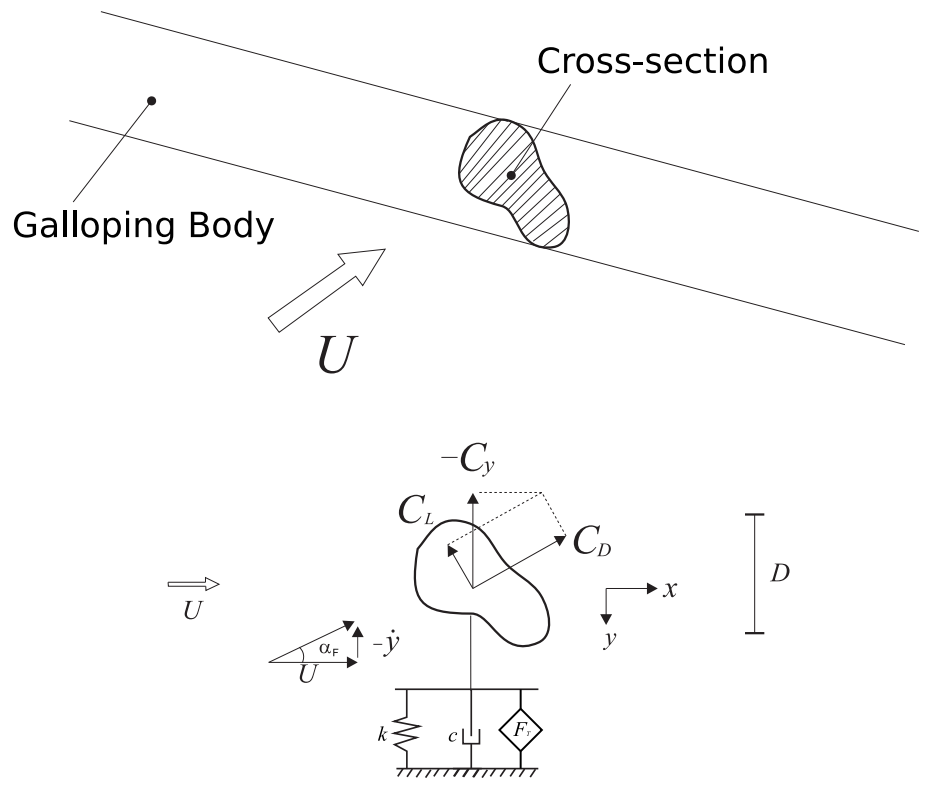

Figure 4.3: Schematics of the fluid forces appearing on the galloping body and of the induced angle of attack obtained through the quasi-steady hypothesis.

Appropriate balance between inertia, damping, stiffness, backlash forces from the transducer, and fluid forces in the system gives the following ordinary differential equation:

$$
m \ddot{y}+c \dot{y}+k y=\frac{1}{2} \rho U^{2} D C_{y}-F_{T},
$$

where $y$ denotes the transverse position of the prism, $\rho$ is the fluid density, $U$ is the undisturbed velocity of the incident flow, $D$ is the characteristic dimension of the prism prone to gallop normal to the flow, $C_{y}$ is the instantaneous fluid force coefficient in the transverse direction to the incident 


\begin{tabular}{lcccl}
\hline Cross-section & $a_{1}$ & $a_{3}$ & $\eta_{F}$ & Source \\
\hline Square & 2.3 & -18 & 0.05 & Parkinson and Smith $(1964) ; R e \approx 5 \cdot 10^{4}$ \\
Isosceles triangle $\left(\delta=30^{\circ}\right)$ & 2.9 & -6.2 & 0.25 & Alonso et al. $(2007) ; R e \approx 10^{5}$ \\
D-section & 0.79 & -0.19 & 0.54 & Novak and Tanaka $(1974) ; R e \approx 10^{5}$ \\
Isosceles triangle $\left(\delta=53^{\circ}\right)$ & 1.9 & -6.7 & 0.09 & Burton et al. $(2001) ; R e \approx 10^{4}$ \\
\hline
\end{tabular}

Table 4.1: Static fluid dynamic characteristics of different croos-sections.

flow, $F_{T}$ is the backlash transducer force per unit length in the $y$ direction and, finally, the dot symbol stands for differentiation with respect to physical time $t$. As above commented, damping and stiffness forces have been considered linear. For an introduction about how non-linear structural effects can be introduced and mathematically modeled the reader is referred to Lee et al. 2011.

\subsubsection{Transverse fluid force coefficient $C_{y}$}

As previously stated, in order to describe the transverse fluid force $C_{y}$, the quasi-steady hypothesis is resorted to, since it is assumed that the characteristic timescale of the prism oscillation $T_{M}$ (of order $2 \pi(m / k)^{1 / 2}$ ) is much larger than the characteristic timescale of the flow $T_{R}$ (of order $D / U$ ) and much larger than the characteristic timescale of vortex shedding $f_{S t}^{-1}\left(D / \mathrm{USt}^{-1}\right)$. Then, for a given crosssection, the fluid force can be considered to be quasi-static as well as being only dependent on the instantaneous attitude of the prism with respect to the flow, which can be described by the motioninduced angle of attack $\alpha_{F}$. Since the motion-induced angle of attack is given by $\alpha_{F}=-\tan ^{-1}(\dot{y} / U)$, the fluid force can be introduced as an empirical function of $\dot{y}$, which can be approximated by a polynomial when the static variation of $C_{y}$ with $\alpha_{F}$ is known (Blevins, 1990). Observe that $C_{y}$ can be related to the lift $C_{L}$ and drag $C_{D}$ coefficients since $C_{y}=-\left(C_{L}+C_{D} \tan \alpha_{F}\right) / \cos \alpha_{F}$ (see Fig. 4.3). For our purposes a cubic polynomial can be considered accurate enough to approximate the vertical fluid force coefficient ${ }^{3}$,

$$
C_{y}=a_{1} \frac{\dot{y}}{U}+a_{3}\left(\frac{\dot{y}}{U}\right)^{3},
$$

where $a_{1}$ and $a_{3}$ are the empirical coefficients to fit by a polynomial the $C_{y}$ versus $\tan \left(\alpha_{F}\right)$ dependence measured in static tests. $a_{1}$ and $a_{3}$ are dependent mainly on the cross-section geometry of the prism. The linear coefficient $a_{1}$ is the slope of the vertical fluid force coefficient at zero angle of attack, and therefore it is necessary for it to be $a_{1}>0$ in order to obtain a galloping response (that is, linearized fluid forces act as a negative damping term when $a_{1}>0$ ). $a_{3}$ accounts for the nonlinear effects and it has to be negative. With a third order polynomial for $C_{y}$, one can obtain with fairly good precision the critical velocity at which galloping begins as well as the asymptotic solution for the amplitude of oscillation of the prism and power extraction as a function of the velocity of the flow. However, note that with a third order polynomial to describe the fluid dynamic transverse force coefficient no hysteresis phenomena can be described and hence will be disregarded. In Table 4.1, a selection of different cross-sections and their transverse fluid force coefficients $a_{1}$ and $a_{3}$ are presented as a reference. In Section 4.6, a method is proposed to modify $C_{y}$ by changing actively the galloping body's orientation with respect to the flow. As will be shown, in this case $a_{1}$ and $a_{3}$ will be dependent on the external imposed rotation law.

\subsubsection{Analytical approximation}

In the forthcoming, the results obtained by Barrero-Gil et al. (2010) in the field of energy harvesting from transverse galloping are reviewed briefly as they constitute the basis for the discussion of the

\footnotetext{
3 Blevins (1990)
} 
rest of the chapter. They considered a generic viscous damper in order to extract the power from the current and took the backlash force to be $F_{T}=c_{E} \dot{y}$. Eq. 4.2 can be be expressed in non-dimensional variables taking as characteristic magnitudes the transverse dimension $D$ and natural frequency of oscillation $\omega_{N}=(k / m)^{1 / 2}$, which yields the following non-dimensional variables $Y=y / D, \tau=\omega_{N} t$, being $\tau$ the non-dimensional time. One may then rewrite the dynamical equation in dimensionless form as

$$
Y^{\prime \prime}+2\left(\zeta+\zeta_{E}\right) Y^{\prime}+Y=\frac{U^{* 2}}{2 m^{*}}\left(a_{1} \frac{Y^{\prime}}{U^{*}}+a_{3}\left(\frac{Y^{\prime}}{U^{*}}\right)^{3}\right),
$$

where prime denotes differentiation with respect to the dimensionless time, $\zeta=c /\left(2 m \omega_{N}\right)$ and $\zeta_{E}=c_{E} /\left(2 m \omega_{N}\right)$ are the dimensionless mechanical damping and the equivalent generic transducer dimensionless damping term respectively, $U^{*}=U /\left(\omega_{N} D\right)$ is the reduced velocity and $m^{*}=m /\left(\rho D^{2}\right)$ is the mass ratio.

Once the static curve for $C_{y}$ (thus, $a_{1}$ and $a_{3}$ ) are known, Eq. 4.4 can be solved either numerically or through asymptotic methods if the non-linearities are small enough, i.e. when the nonlinearity of the fluid forcing term is weak compared to the inertial and stiffness forces (that is, for $m^{*} U^{*} \gg 1$, which is usual when the fluid in consideration is air). In this case, the solution of the LCO will be a quasi-harmonic oscillation of amplitude $A^{*}$. This case is typical for civil structures affected by aerodynamic forces, where the mass ratio $m^{*}$ is of order $10^{3}$. For the rest of the chapter, solutions of the dynamical equation (namely Eq. 4.4), will be solved through the Harmonic Balance Method. The Harmonic Balance Method can be used when the solution of the problem is of a periodic nature. In practice, this means that an "ansatz" has to be made that needs to be verified after the said solution has been obtained. However, when compared to perturbation methods that do not require a priori restrictive hypothesis, this may seem kind of a drawback. On the other hand, tailoring the solution method to the specific nature of the solution itself has practical advantages regarding the numerical solution procedure; and this is the main reason why this approach has been chosen for this study. It is, nevertheless, to be mentioned that the thesis director, see Barrero-Gil et al.(2010), has already used the Krylov-Bogoliubov perturbation method to address this same transverse galloping problem. In that case, one may assume that the steady-state movement response is sinusoidal, $Y=A^{*} \sin \left(\omega^{*} \tau\right)$, where $\omega^{*}=\omega / \omega_{N}$. Substituting this into Eq. 4.4, taking into account that $\cos ^{3}(\omega \tau) \approx 3 \cos (\omega \tau) / 4$, (i.e. higher order terms in $\cos \left(3 \omega^{*} \tau\right)$ are neglected) which is valid when the system is narrow-band in frequency such as for the case in study, and equating sine and cosine terms, after some algebra one gets,

$$
\begin{gathered}
A^{*}=\left(\frac{4 U^{*}}{3 a_{3}}\left(4 m^{*} \zeta_{T}-a_{1} U^{*}\right)\right)^{1 / 2}, \\
\omega^{*}=1 .
\end{gathered}
$$

Note that the frequency of oscillations (with the hypothesis that have been posed) is always equivalent to the natural frequency of oscillation and independent of the reduced velocity. This is caused by the forces considered in the quasi-steady model being in phase with the velocity of displacement $Y^{\prime}$.

In Eq. 4.5a, an equivalent total damping term has been defined as $\zeta_{T}=\zeta+\zeta_{E}$. Galloping oscillations are expected to happen when a critical reduced velocity is overcome $\left(A^{*}>0\right)$,

$$
U_{g}^{*}=\frac{4 m^{*} \zeta_{T}}{a_{1}}
$$

Once galloping oscillations appear, these increase with the reduced velocity monotonously. For 
high enough reduced velocity, Eq. 4.5a can be approximated as an asymptotic solution yielding

$$
A_{\infty}^{*}=U^{*} \sqrt{-\frac{4 a_{1}}{3 a_{3}}}
$$

which is valid when $a_{1} U^{*} \gg 4 m^{*} \zeta_{T}$. Note that the final asymptotic solution is only a function of the cross-section geometry through the parameters of the transverse force coefficient $a_{1}$ and $a_{3}$. In particular, the slope of the asymptotic solution for $A^{*}$ is proportional to the value of $\tan \left(\alpha_{F}\right)$ where $C_{y}=0$. Those cross-sections that present a higher value of $\alpha_{F}$ where the transverse force coefficient cuts the zero axis have higher asymptotic slope (for the proposed cross-sections in Fig. 4.1b and Table 4.1, the D-section presents the steepest slope.

\subsubsection{Energy harvesting efficiency}

The mean power harnessed at the viscous damper $c_{E}$ can be defined as

$$
P_{E}=\frac{1}{T} \int_{0}^{T} F_{T} \dot{y} d t=\frac{1}{T} \int_{0}^{T} c_{E} \dot{y}^{2} d t
$$

where $T$ is an averaging time (e.g. the period of oscillation for purely sinusoidal oscillations). A conversion factor (or efficiency) can be defined as the mean power $P_{E}$ dissipated at the viscous damper divided by the total power in the flow per unit length as

$$
\eta_{E}=\frac{P_{E}}{\frac{1}{2} \rho U^{3} D}
$$

The efficiency of energy extraction, as described in Eq. 4.9, is presented in the same way as in Barrero-Gil et al. (2010), or Grouthier et al. (2013), where the power harvested is compared to the power available in the flow in a section equivalent to the diameter of the prism. However, other ways of defining such efficiency are possible. For instance, the efficiency can be defined with the power harvested compared to the power available in the flow in a section equivalent to the space swept by the prism during its oscillations. With the later formulation, one could explore the existence of an equivalent to the Betz limit for horizontal-axis turbines. However, the main caveat is that it relies on the definition of the swept area of the prism which is itself a result of the problem. Note that both efficiencies might yield different results of optimal configuration as one stresses the importance of the maximum power extracted while the other one stresses the importance of maximizing the energy harvested per unit length in the transverse direction. Note that the definition of efficiency as considered in Eq. 4.9 is not a thermodynamical efficiency, thus, values of $\eta_{E}$ could yield values higher than 1. From Eqs. 4.5, 4.8 and 4.9 it follows that

$$
\eta_{E}=\frac{4 m^{*} \zeta_{E}\left(A^{*} \omega^{*}\right)^{2}}{U^{* 3}}=\frac{8 m^{*} \zeta_{T}}{3 a_{3} U^{* 2}}\left(4 m^{*} \zeta-a_{1} U^{*}\right),
$$

which relates the efficiency of energy extraction as a function of different parameters involved in the transverse galloping phenomenon. Maximum hypothetical energy harvesting efficiency can be obtained by differentiating Eq. 4.10 with respect to the reduced velocity and equating to 0, from which one obtains the maximum efficiency for the fluid-elastic power extraction,

$$
\eta_{E}^{\max }=-\frac{a_{1}^{2}}{6 a_{3}} \frac{1}{1+\zeta / \zeta_{E}}
$$

at a reduced velocity of $U_{\eta_{E}^{\text {max }}}^{*}=2 U_{g}^{*}$. The second factor is related to the loss of power as structural 
damping compared to the fraction of power extracted as electricity. In the limit, when $\zeta_{E} \gg \zeta$

$$
\eta_{F}=-\frac{a_{1}^{2}}{6 a_{3}} .
$$

The maximum efficiency as derived in Eq. 4.12, gives the maximum achievable efficiency when it is considered that there no losses of any kind and when the transducer acts like a pure viscous damper $\left(F_{T}=c_{E} \dot{y}\right)$. As can be seen, the maximum efficiency for the fluid-elastic power extraction is only dependent on the geometry of the cross-section of the galloping prism (through $a_{1}$ and $a_{3}$ coefficients). As a reference, Table 4.1 presents the maximum efficiency expected $\left(\eta_{F}\right)$ for several cross-sections. Notoriously, cross-sections that present maximum efficiency are those that present the largest area beneath the $C_{y}$ curve as a function of $\tan \left(\alpha_{F}\right)$.

In the forthcoming, variations to the previous results for the dynamic behavior and energy harvesting of transverse galloping are presented. These variations are due to the different transducer mechanisms employed to produce electrical energy. In first place, an electromagnetic transducer is analyzed. Secondly, an analysis is made considering a mechanical to electrical converter via piezoelectric means. 


\subsection{Optimal electromagnetic energy extraction}

Here, a forward step is presented and developed in order to model the transverse galloping dynamics when an electromagnetic generator is used to produce electrical current. To this end, the model developed at Barrero-Gil et al. (2010) and in Section 4.2 is now coupled with a mathematical model of an electromagnetic generator. A fully coupled electro-fluid-elastic model for electromagnetic energy harvesting from transverse galloping is thus obtained and presented here. The electromagnetic generator is modeled by an equivalent electrical circuit and the power is dissipated at a generic electrical load resistance. Two different levels of simplification have been made depending on the comparison between the characteristic electrical and mechanical timescales. The effect of the electrical resistance load on the energy harvested is studied theoretically. On the practical side, this result can be helpful to design tracking-point strategies to maximize energy harvesting for variable flow velocity conditions.

Part of the results presented in this Section 4.3 have been published in Optimal electromagnetic energy extraction from transverse galloping, Journal of Fluids and Structures, Volume 51, November 2014, Pages 281-291.

\subsubsection{Mathematical model}

a)

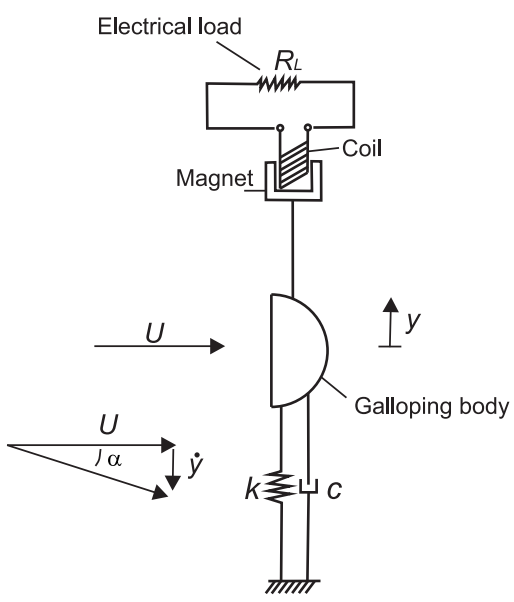

b)

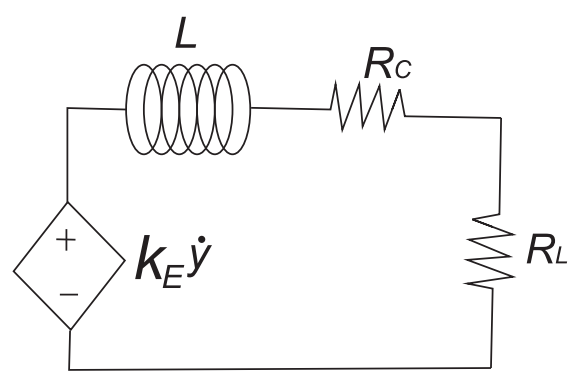

Figure 4.4: (a) Sketch of the spring-mounted galloping body in cross-flow and linked to an electromagnetic generator. (b) Equivalent electrical circuit of the electromagnetic generator.

Let us consider an equivalent electrical model to modelize the electromagnetic generator (see Fig. 4.4b; El-Hami et al. (2001), Rizzoni and Hartley, 2000), which gives a relationship between the main parameters of the electromagnetic generator and the induced electromagnetic force $F_{T}$. This equivalent circuit for the generator is modeled as

$$
\begin{gathered}
\left(R_{L}+R_{c}\right) i+L \frac{d i}{d t}=k_{E} \frac{d y}{d t}, \\
F_{T}=k_{E} i
\end{gathered}
$$

where $R_{L}$ is the resistance of the load connected to the generator, $R_{C}$ is the internal resistance of the coil, $L$ its inductance, $k_{E}$ is an electromechanical coupling constant determined by the flux density of the magnetic field and the number of turns and length of the coil, and $i$ is the intensity circulating through the coil which constitute a simplified model of an electromagnet generator. Introducing dimensionless variables $I=i / I_{c}$, being $I_{c}=m \omega_{N}^{2} D / k_{E}$, one may rewrite Eq. 4.4 along Eqs. 4.13a- 
4.13b in dimensionless form,

$$
\begin{gathered}
Y^{\prime \prime}+2 \zeta Y^{\prime}+Y=\frac{U^{* 2}}{2 m^{*}}\left(a_{1} \frac{Y^{\prime}}{U^{*}}+a_{3}\left(\frac{Y^{\prime}}{U^{*}}\right)^{3}\right)-I, \\
\beta I^{\prime}+I=2 \zeta_{E} Y^{\prime}
\end{gathered}
$$

where prime denotes differentiation with respect to the dimensionless time, $\beta=\left(L \omega_{N}\right) /\left(R_{L}+R_{C}\right)$ is a relationship between the characteristic electrical and mechanical time and $\zeta_{E}$ is a dimensionless damping coefficient which is a result of the coupling of the dynamics of the prism and the electromagnetic generator

$$
\zeta_{E}=\frac{k_{E}^{2}}{2 m \omega_{N}\left(R_{L}+R_{C}\right)} .
$$

In this case, the discussion will regard the useful power extracted and converted into electrical power dissipated in the electrical load, defined as

$$
P_{E}=\frac{1}{T} \int_{0}^{T} R_{L} i^{2} d t
$$

The electromagnetic efficiency $\eta_{E}$ can be defined as the mean power $P_{E}$ dissipated at the electrical load divided by the total power in the flow per unit length as

$$
\eta_{E}=\frac{P_{E}}{\frac{1}{2} \rho U^{3} D}
$$

The system given by Eqs. 4.14a to 4.17 allows to obtain the galloping response and the power dissipated at $R_{L}$ as a function of the mechanical parameters $\left(m^{*}, \zeta, \omega_{N}\right)$, geometrical and flow parameters $\left(U^{*}, a_{1}, a_{3}, D\right)$, and parameters of the electromagnet generator $\left(k_{E}, \beta, R_{C}\right)$. The discussion will be mainly focused on the optimization of $\eta_{E}$. Furthermore, an analytical approach is presented in the following section, which can be quite useful in order to establish design rules for $R_{L}$ in order to extract maximum power.

\subsubsection{Galloping response, energy efficiency, and optimal electrical load re- sistance}

\subsubsection{Low frequency oscillations. $\beta \ll 1$.}

If $\beta \ll 1$ is considered, it means that the effect of the inductance on the dynamic response is negligible and in this case, an easy simplification can be considered, that is $i=2 \zeta_{E} I_{c} Y^{\prime}$ (see Eq. 4.14b), and Eq. 4.14a is then

$$
Y^{\prime \prime}+2 \zeta Y^{\prime}+Y=\frac{U^{* 2}}{2 m^{*}}\left(a_{1} \frac{Y^{\prime}}{U^{*}}+a_{3}\left(\frac{Y^{\prime}}{U^{*}}\right)^{3}\right)-2 \zeta_{E} Y^{\prime} .
$$

Proceeding similarly as for Eq. 4.5a (through the Harmonic Balance Method), one obtains the dynamical solution for the galloping system as

$$
\begin{gathered}
A^{*}=\left(\frac{4 U^{*}}{3 a_{3}}\left(4 m^{*} \zeta_{T}-a_{1} U^{*}\right)\right)^{1 / 2}, \\
\omega^{*}=1
\end{gathered}
$$

where $\zeta_{T}=\zeta+\zeta_{E}$. Observe that galloping oscillations $\left(A^{*}>0\right)$ are only possible when $U^{*}>U_{g}^{*}=$ $4 m^{*} \zeta_{T} / a_{1}$. It is important to remark that the value of $U_{g}^{*}$ depends on the load resistance $R_{L}$ (via $\left.\zeta_{E}\right)$, which is a primary result of the ongoing study. For this case, the frequency of oscillation is not 
modified (as in Eq. 4.5b) as the equivalent circuit of the electromagnetic generator for $\beta \ll 1$ acts like a pure viscous damper.

The mean power per unit length is (see Eq. 4.16)

$$
P_{E}=\frac{4}{2} R_{L} \zeta_{E}^{2} I_{C}^{2} A^{* 2} \omega^{* 2}=\frac{8 R_{L} \zeta_{E}^{2} I_{C}^{2} U^{*}}{3 a_{3}}\left(4 m^{*} \zeta_{T}-a_{1} U^{*}\right)
$$

and the efficiency

$$
\eta_{E}=\frac{16 R_{L} \zeta_{E}^{2} I_{C}^{2}}{3 a_{3} \rho \omega_{n}^{3} D^{4} U^{* 2}}\left(4 m^{*} \zeta_{T}-a_{1} U^{*}\right)
$$

Eq. 4.21 can be rewritten in order to express all variables non-dimensionally as

$$
\eta_{E}=\frac{8 m^{*} \zeta_{E}}{3 a_{3}\left(1+R_{C} / R_{L}\right) U^{* 2}}\left(4 m^{*} \zeta_{T}-a_{1} U^{*}\right)
$$

\subsection{Maximum efficiency achievable for fixed $R_{L}$.}

The conversion factor (or electromagnetic efficiency) is a function of $R_{L}$ and $U^{*}$, so in order to obtain the maximum efficiency, it is necessary to obtain the partial derivatives of Eq. 4.22 with respect to $U^{*}$ and $R_{L}$, equate to zero and solve the system of 2 equations and 2 unknowns,

$$
\begin{aligned}
& \frac{\partial \eta_{E}}{\partial U^{*}}=0 \\
& \frac{\partial \eta_{E}}{\partial R_{L}}=0 .
\end{aligned}
$$

In first place, if $R_{L}$ is considered to be a fixed value, then only Eq. 4.23a applies. By solving Eq. 4.23a it is possible to retrieve the reduced velocity at which the maximum efficiency is achieved, giving a value of reduced velocity of $2 U_{g}^{*}$,

$$
U_{\eta_{E}^{\max }}^{*}=2 U_{g}^{*}=\frac{8 m^{*}\left(\zeta+\zeta_{E}\right)}{a_{1}}
$$

Notoriously, this result was experimentally confirmed by Hémon et al. (2016) where rigid prisms were flexibly mounted in a wind tunnel and the energy harvesting was performed by a series of magnets mounted on the oscillating prism so that they passed close to the face of a coil-core system externally fixed. Substitution of Eq. 4.24 into Eq. 4.22 allows to get the maximum efficiency achievable when $R_{L}$ is fixed, given by

$$
\eta_{E}^{\max }=-\frac{a_{1}^{2}}{6 a_{3}}\left(\frac{1}{1+R_{C} / R_{L}}\right)\left(\frac{1}{1+\zeta / \zeta_{E}}\right),
$$

which relates the maximum achievable efficiency with the cross-section geometry of the prism $\left(a_{1}\right.$ and $\left.a_{3}\right)$, the ratio between the electrical resistances $R_{C} / R_{L}$, and the ratio between the parasite damping and the electrical damping $\zeta / \zeta_{E}$. Thus, if $R_{C}$ and $\zeta$ are equal to zero, the maximum efficiency from Barrero-Gil et al. (2010) (or Eq. 4.12) is recovered.

A universal curve for the efficiency can be introduced using two new normalized variables, $\tilde{\eta}=$ $\eta_{E} / \eta_{E}^{\max }$ and $\tilde{U}=U^{*} / U_{g}^{*}$ and substituting them into Eq. 4.22

$$
\tilde{\eta}=-\frac{4(1-\tilde{U})}{\tilde{U}^{2}} .
$$

With these re-normalized variables, it is possible to collapse the efficiency curves for all electrical/mechanical configurations into a single curve (Eq. 4.26) providing information about the transverse galloping response over a range of reduced incident velocities. From Fig. 4.5, once galloping is started $(\tilde{U}=1)$, the normalized efficiency increases rapidly with the normalized reduced velocity until a value 


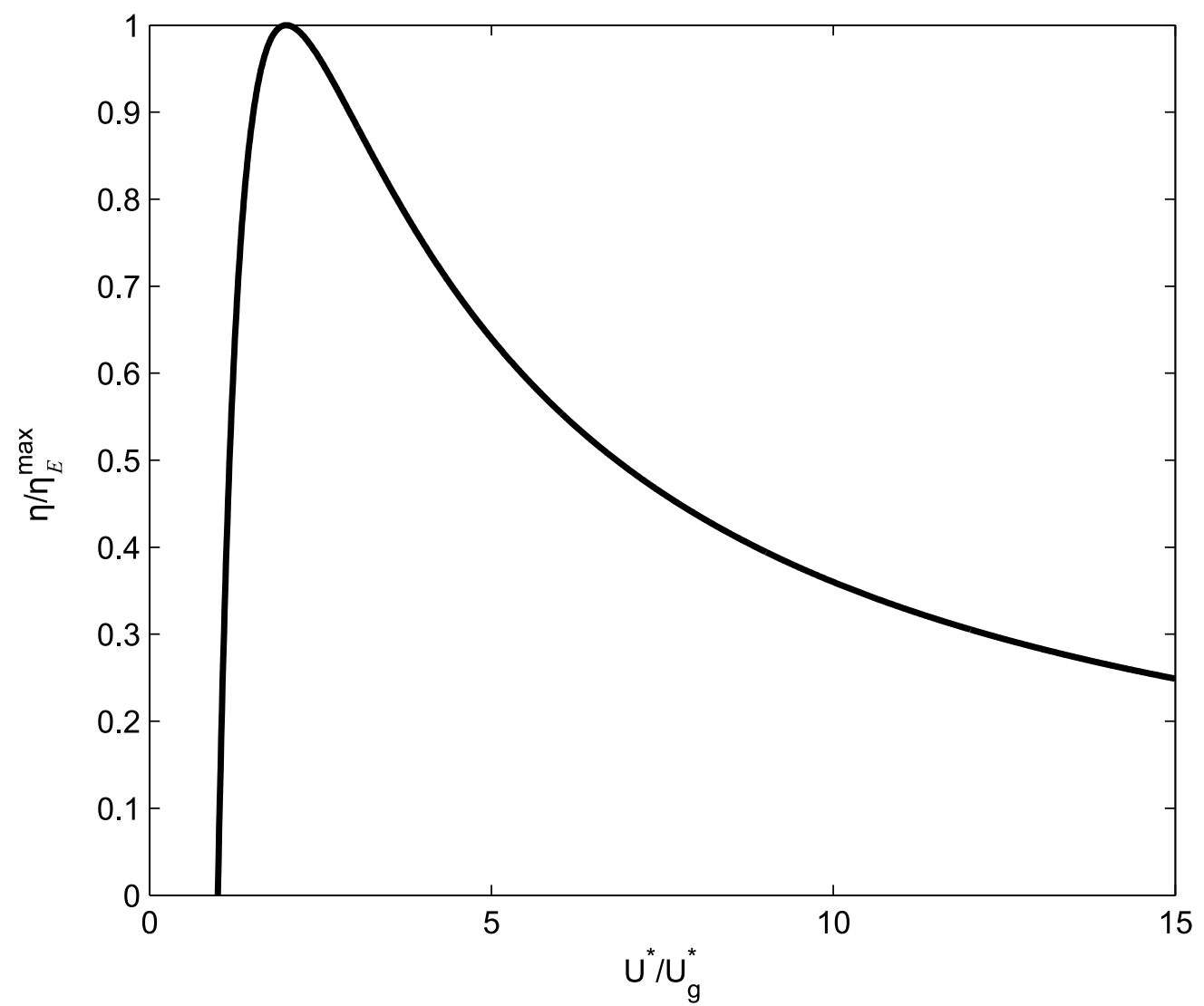

Figure 4.5: Universal plot of the efficiency versus the flow velocity.

of $\tilde{U}=2$ is reached obtaining a value of the normalized efficiency of $\tilde{\eta}=1$, then the normalized efficiency diminishes slowly with $\tilde{U}$.

Thus, it is important to correctly tune the value of $R_{L}$ so as to modify $U_{g}^{*}$ and correspondingly be at the maximum possible efficiency for each value of the reduced velocity and obtaining a broadband type of response.

\subsection{Optimal electrical load resistance for maximum power transfer at any instant.}

From the point of view of designing a control law capable of harnessing the maximum possible power at any instant (similar to a tracking-point strategy for changing flow velocity conditions), $R_{L}$ has to be consequently modified depending on $U^{*}$. In this case, it is only necessary to solve Eq. $4.23 \mathrm{~b}$ and by doing so, it is possible to obtain the electric load that maximizes the power harvested for each value of the reduced velocity

$$
R_{L o p}=\frac{p}{q}+\left(\left(\frac{p}{q}\right)^{2}+R_{C}\left(R_{C}-\frac{p}{q}\right)\right)^{1 / 2}
$$

where $p=4 m^{*} k_{E}^{2} /\left(2 m \omega_{N}\right)$ and $q=a_{1} U^{*}-4 m^{*} \zeta$.

Eq. 4.27 gives the optimal load electrical resistance as a function of the reduced velocity, geometry of the galloping body $\left(a_{1}\right)$, the mechanical parameters of the galloping system $\left(m, m^{*}, \zeta\right.$, and $\left.\omega_{N}\right)$, and electric properties $k_{E}$ and $R_{C}$ of the electromagnet. When $R_{L}$ is large compared to $R_{C}$, which should be a usual condition from the practical point of view, Eq. 4.27 is further simplified and $R_{L o p}$ approximates to

$$
R_{L o p}=\frac{2 p}{q}=\frac{4 m^{*} k_{E}^{2}}{m \omega_{N}\left(a_{1} U^{*}-4 m^{*} \zeta\right)},
$$

Observe that this equation suggests that $R_{L o p}$ scales as $U^{*-1}$ for large values of $U^{*}$. 
If the resistance $R_{L}$ is tuned as suggested at Eq. 4.27 for each reduced velocity, then the maximum possible power is being transferred from the flow to the electrical load. Regarding the issue of power transfer to the electrical generator, it is in order to refer, in broad terms, to the Maximum Power Transfer Theorem. This theorem states that for a given constant voltage supply, the maximum power transferred to the electrical load $R_{L}$ occurs whenever $R_{L}=R_{C}$. In practice, this theorem suggests a way to solve the actual engineering problem of tuning the generator parameters so as to optimize power transfer. However, in the case presented in this article, the Maximum Power Transfer Theorem cannot be applied straightforwardly because the supply voltage is not constant. In particular, voltage behavior is sinusoidal and it depends on both the reduced velocity and $R_{L}$ itself. Thereby, actual tuning of the generator parameters requires solving Eq. $4.23 \mathrm{~b}$ as previously stated. Nevertheless, it is to be noted that in the asymptotic limit of very large reduced velocity the voltage becomes less and less dependent on load resistance, see Eq. 4.22, and therefore, in this distinguished limit, the relation provided by the classical Maximum Power Transfer Theorem is recovered.

From the practical side, the variation of the load resistance could be done using a DC/DC converter capable of varying the equivalent electric load the galloping prism sees in order to always capture the maximum energy for the whole range of reduced velocities. This is a usual application in wind turbines with battery charging applications such at De Broe et al. (1999) and at Moore and Beukes (2004). A Maximum Power Point Tracking (MPPT) system can be also used. This system can be fed with a look-up table with the actuation map as obtained in Eq. 4.22 or by using algorithms which don't use any prior information and by using a control system they automatically obtain the optimal load resistance as proposed in Koutroulis and Kalaitzakis (2006).

Finally, there is a global maximum absolute efficiency point which fulfills both Eqs. 4.23a and 4.23b given by

$$
R_{L \max }^{G}=\sqrt{R_{C}\left(R_{C}+\frac{p}{4 m^{*} \zeta}\right)}
$$

which is independent of $a_{1}$ and $a_{3}$. Once the value of $R_{L \max }^{G}$ that maximizes the efficiency is known, the value of the reduced velocity at which such maximum happens is also determined,

$$
U_{\max }^{* G}\left(R_{L \max }^{G}\right)=2 U_{g}^{*}\left(R_{L \max }^{G}\right)=\frac{8 m^{*}\left(\zeta+\zeta_{E}\left(R_{L \max }\right)\right)}{a_{1}},
$$

and

$$
\eta_{E}^{G, \max }=-\frac{a_{1}^{2}}{6 a_{3}}\left(\frac{1}{1+R_{C} / R_{L \max }^{G}}\right)\left(\frac{1}{1+\zeta / \zeta_{E}\left(R_{L \max }^{G}\right)}\right) .
$$

Previous equation indicates that the maximum achievable efficiency using an electromagnetic generator is equivalent to the maximum fluid-elastic efficiency $\eta_{F}=-a_{1}^{2} / 6 a_{3}$ (recovering the solution from Eq. 4.12, which is only dependent on the cross-section) modulated by two conversion factors, one related to the loss of energy in the internal resistance of the coil and another related to the loss of energy as parasitic damping.

\subsection{Analysis of a possible practical realization}

It may be now of interest to discuss a potential practical realization for small power production (approximately 50 Watts) that illustrates the theoretical results obtained previously. It consists in a spring-mounted prism with a D-type cross-section which is linked to the magnet of an electromagnet in such a way the magnet can oscillate relative to the coil; the prism is under the action of an airstream and the electromagnet has a maximum rated power of 50 Watts. Physical properties of the galloping body and the electromagnet generator are listed in Table 4.2.

Fig. 4.6a shows the optimal load electrical resistance $R_{L o p}$ that should be connected at the electromagnet at each wind speed (computed from Eq. 4.27). Note how $R_{L o p}$ diminishes as wind speed 
Table 4.2: Physical properties of galloping body and the electromagnet generator. Values of the coefficients $a_{1}$ and $a_{3}$ are taken from Barrero-Gil et al. (2010).
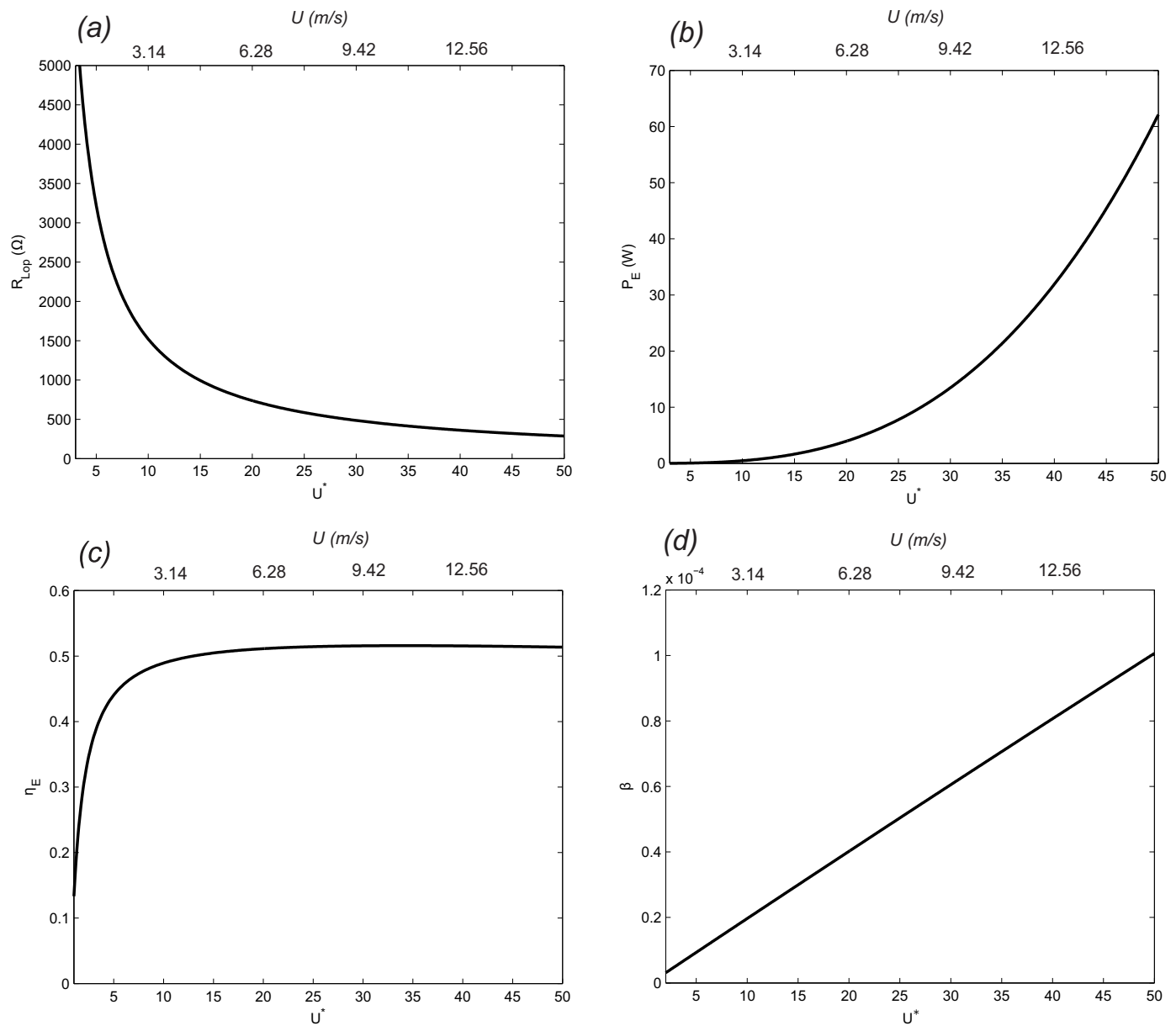

Figure 4.6: Variation with the reduced velocity $U^{*}$ of the optimal electrical load resistance (a), output electrical power (b), efficiency (c), and $\beta(\mathrm{d})$.

increases; note also that $R_{L o p}$ is very large with respect to $R_{C}$ (for $U^{*}=50, R_{L o p}$ is still 24 times larger than $R_{C}$ ). Fig. $4.6 \mathrm{~b}$ shows the electrical power variation with the wind speed when the load electrical resistance is optimally tuned and Fig. 4.6c the efficiency behavior with the reduced velocity (wind speed) when the load electrical resistance is optimally tuned; observe that because of non-zero mechanical parasite damping (remember that $\zeta=0.002$ ) and electrical losses at $R_{C}$, the maximum efficiency has diminished from the ideal value of $0.54\left(-a_{1}^{2} /\left(6 a_{3}\right)\right)$ down to 0.51 ; this absolute maximum value has been achieved with $R_{L \max }^{G}=420 \Omega$ at $U_{\max }^{* G}=34$ as predicted by Eqs. 4.29 and 4.31 . Because of the optimal choice of $R_{L o p}$ for each wind speed, the efficiency dependence with the reduced velocity is diminished significantly and a broadband-type behaviour can be observed. The idea is that, as $U^{*}$ increases, the electrical load resistance is diminished accordingly to Eq. 4.27 in order to increase the electrical damping and thus increase $U_{g}^{*}$ in such a way that $U^{*}$ is close to $2 U_{g}^{*}$ at any instant, making possible to maintain the efficiency at high level for a larger range of reduced velocities. From the practical side this is a remarkable result since it is possible to get a low critical velocity of galloping and, at the same time, get high efficiency in a large range of reduced velocities. Observe that the potential to enlarge the range where efficiency is maintained at a high level is limited by $R_{C}$, since it 
imposes an upper limit for the electrical damping. Finally Fig. 4.6d shows the value of $\beta$ variation with reduced velocity in order to check that $\beta$ is always much lower than 1 .
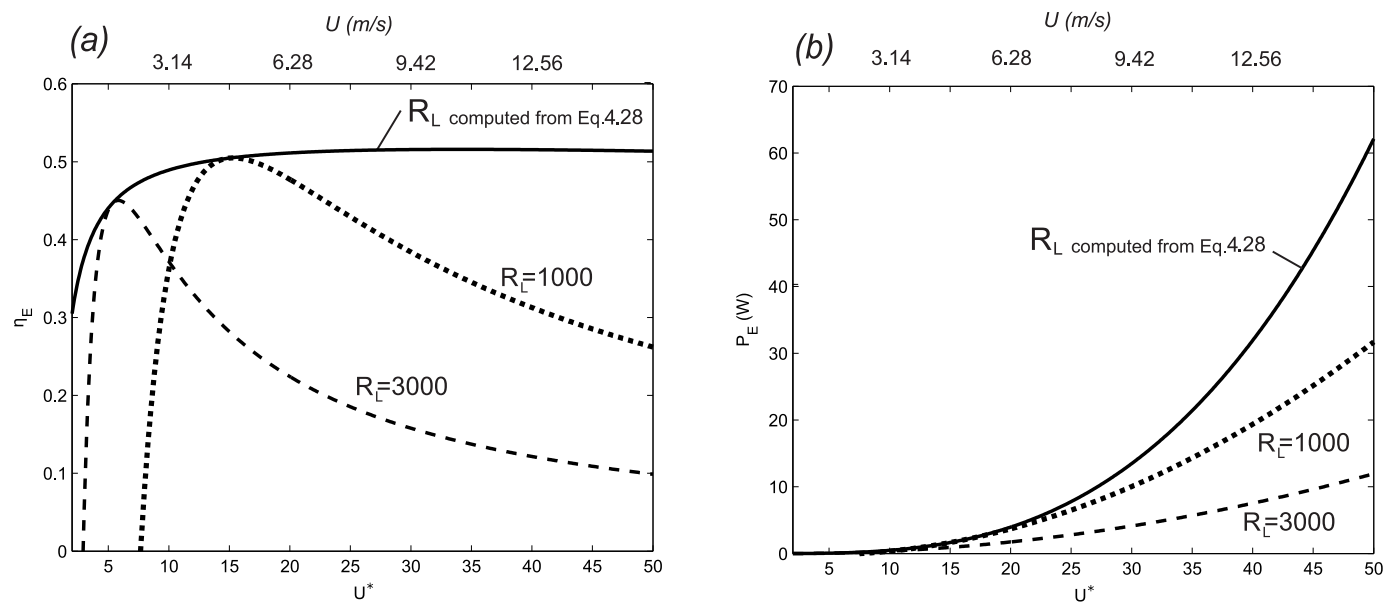

Figure 4.7: (a) Efficiency variation with reduced velocity for optimal electrical load resistance (solid line) and for two different fixed values of the electrical load resistance (dashed lines). White circles denote the local maximum efficiency for a given $R_{L}$ whereas black circle denotes the absolute maximum efficiency. Note that the efficiency variation with reduced velocity for optimal electrical resistance (solid line) is the envelope of all local maximum efficiency points. (b) Output power variation with reduced velocity for optimal electrical load resistance (solid line) and for two different fixed values of the electrical load resistance (dotted line).

Figs. $4.7 \mathrm{a}$ and $4.7 \mathrm{~b}$ highlight the advantage associated to optimally choosing $R_{L}$ at any value of the reduced velocity. Fig. 4.7a shows the efficiency dependence with the reduced velocity when the electrical load resistance is optimal at any reduced velocity, as well as the efficiency dependence for two cases where the the electrical load resistance takes a constant value. When $R_{L}$ takes a constant value, as the reduced velocity increases the efficiency increases until it reaches a maximum value (dependent on $R_{L}$ ). Further increase in $U^{*}$ results in a decrease in the efficiency which approaches to zero, so that the efficiency of energy conversion is in high levels only in a small range of reduced velocities: at low reduced velocities for large $R_{L}$ values and high reduced velocities for small $R_{L}$ values. However, when $R_{L}$ changes optimally with the reduced velocity, the efficiency increase with $U^{*}$ until reach a final value of high efficiency and then remains tenaciously at high levels almost independently of the reduced velocity, which shows the advantages of adjusting continually and optimally the electrical resistance. In addition, when $R_{L}$ is optimally chosen the reduced velocity at which galloping starts (and power output begins) is low, but when $R_{L}$ takes a fixed value galloping oscillations can start at large reduced velocities (observe in Fig. 4.6a that efficiency curve starts around $U^{*}=3$ for $R_{L}=3000$ and $U^{*}=7.5$ for $R_{L}=1000$ ). Finally, it is apparent from Fig. 4.7a that, as discussed earlier, a re-normalization exits and all curves for $R_{L}$ constant collapses into a single one when redrawn in normalized variables $\tilde{U}$ and $\tilde{\eta}$.

In terms of electrical power output, the advantage of adjusting continually the electrical resistance $R_{L}$ with the reduced velocity over take a fixed value is also clear, as is can be seen in Fig. 4.7b.

The energy harvesting efficiency response obtained can be compared to other devices. For example, at Abdelkefi et al. (2013c), the same galloping model as presented here was taken into account, but the coupled electrical model used was a piezoelectric one. Results of efficiency for piezoelectric coupling leads to values much lower than the ones presented in this article, between 0.01 and 0.03. An experimental validation of electromagnetic energy extraction using wake galloping (not transverse galloping) through wind tunnel testing was carried out by Jung and Woo-Lee (2011); they measured efficiencies up to 0.094. In the VIVACE concept, the energy is generated via electromagnetic conversion and taking advantage from Vortex Induced Vibrations of a circular cylinder; efficiencies up to 0.31 
were reported (see Raghavan 2007) by correctly tuning the load resistance. While VIV is a resonancetype phenomenon where energy can only be harvested from the current for incident reduced velocities close to the resonance one, galloping has a much more broad type of response and by continuously changing $R_{L}$, it is possible to even maintain high efficiency values for broader values of the reduced velocities.

\subsubsection{Moderate frequency oscillations. $\beta<1$.}

In some situations, requirements needed for $\beta \ll 1$ may be not fulfilled, so it is of interest to expand the domain of the analysis in order to look for the inductance effect when $\beta$ cannot be neglected and $\beta<1$. To this end, a perturbation expansion in $\beta$ is considered for the intensity in the electromagnet, $I=I_{0}+\beta I_{1}+\beta^{2} I_{2}$, in such a way that Eq. $4.14 \mathrm{~b}$ may be rewritten as

$$
\beta\left(I_{0}^{\prime}+\beta I_{1}^{\prime}+\beta^{2} I_{2}^{\prime}\right)+\left(I_{0}+\beta I_{1}+\beta^{2} I_{2}\right)=2 \zeta_{E} Y^{\prime} .
$$

Collecting, respectively, zero order terms, $\beta$ and $\beta^{2}$ order terms,

$$
\begin{aligned}
& I_{0}=2 \zeta_{E} Y^{\prime}, \\
& I_{1}+I_{0}^{\prime}=0, \\
& I_{2}+I_{1}^{\prime}=0,
\end{aligned}
$$

and solving one after the other, one may get

$$
I=2 \zeta_{E}\left(Y^{\prime}-\beta Y^{\prime \prime}+\beta^{2} Y^{\prime \prime \prime}\right)
$$

Substituting now Eq. 4.34 into Eq. 4.14a

$$
Y^{\prime \prime}+2 \zeta Y^{\prime}+Y=\frac{U^{* 2}}{2 m^{*}}\left(a_{1} \frac{Y^{\prime}}{U^{*}}+a_{3}\left(\frac{Y^{\prime}}{U^{*}}\right)^{3}\right)-2 \zeta_{E} Y^{\prime}+2 \zeta_{E} \beta Y^{\prime \prime}-2 \zeta_{E} \beta^{2} Y^{\prime \prime \prime} .
$$

The effect of the electrical inductance $(\beta)$ is to introduce a negative added mass (proportional to $\beta$ ) term as well as a negative damping term (proportional to $\beta^{2}$ ) into the dynamics of the galloping prism. Operating in the same way as previously, Eq. 4.35 can be analytically approximated when $m^{*} U^{*}$ is large enough. Assuming that the steady motion response is sinusoidal, $Y=A^{*} \sin \left(\omega^{*} t\right)$, where $\omega^{*}=\omega / \omega_{N}$, and substituting this into Eq. 4.35, after equating sine and cosine term, one gets

$$
\begin{gathered}
A^{*}=\left(\frac{4 U^{*}\left(1-2 \zeta_{E} \beta\right)}{3 a_{3}}\left(4 m^{*} \zeta_{T}-a_{1} U^{*}-2 m^{*} \beta\left(\frac{2 \zeta_{E} \beta}{1-2 \zeta_{E} \beta}\right)\right)\right)^{1 / 2}, \\
\omega^{* 2}=\frac{1}{1-2 \zeta_{E} \beta} .
\end{gathered}
$$

Variations in the frequency of oscillation are caused due to the negative equivalent mass added by the effect of the inductance. Since electrical power is proportional to $A^{* 2} \omega^{* 2}$ (see Eq. 4.16), let us collect $A^{*}$ and $\omega^{*}$

$$
A^{* 2} \omega^{* 2}=\left(\frac{4 U^{*}}{3 a_{3}}\left(4 m^{*} \zeta_{T}-a_{1} U^{*}-2 m^{*} \beta\left(\frac{2 \zeta_{E} \beta}{1-2 \zeta_{E} \beta}\right)\right)\right),
$$

which can be simplified if terms of higher order than $\beta^{2}$ are neglected

$$
A^{* 2} \omega^{* 2}=\left(\frac{4 U^{*}}{3 a_{3}}\left(4 m^{*} \zeta_{T}-a_{1} U^{*}-4 m^{*} \zeta_{E} \beta^{2}\right)\right)+O\left(\beta^{3}\right)
$$


since

$$
\beta \frac{2 \zeta_{E} \beta}{1-2 \zeta_{E} \beta}=2 \zeta_{E} \beta^{2}+O(\beta)^{3} .
$$

Substituting Eq. 4.38 into Eq. 4.16, the electrical power per unit length is given now by

$$
P_{E}^{\beta}=\frac{8 R_{L} \zeta_{E}^{2} I_{C}^{2} U^{*}\left(1-\beta^{2}\right)}{3 a_{3}}\left(4 m^{*} \zeta_{T}-a_{1} U^{*}-4 m^{*} \zeta_{E} \beta^{2}\right),
$$

and the efficiency by

$$
\eta_{E}^{\beta}=\frac{16 R_{L} \zeta_{E}^{2} I_{C}^{2}\left(1-\beta^{2}\right)}{3 a_{3} \rho D \omega_{n}^{3} D^{3} U^{* 2}}\left(4 m^{*} \zeta_{T}-a_{1} U^{*}-4 m^{*} \zeta_{E} \beta^{2}\right),
$$

where, as said, $\beta^{3}$ terms have been neglected.

\subsection{Optimal electrical load resistance}

The optimal electrical load can be obtained by differentiating Eq. 4.41 with respect to $R_{L}$ and equating to zero; this leads to

$$
R_{\text {Lop }}=\frac{p}{q}\left(1-\beta^{2}\right)+\left(\left(\frac{p}{q}\right)^{2}\left(1-\beta^{2}\right)^{2}+R_{C}\left(R_{C}-\frac{p}{q}\left(1-\beta^{2}\right)\right)\right)^{1 / 2},
$$

which is valid up to order $\beta^{3}$. Note that Eq. 4.42 coincides with the result given in the analysis carried out for low frequency oscillations (Eq. 4.27) when $\beta=0$. Fig. 4.8 shows a comparison of

(a)

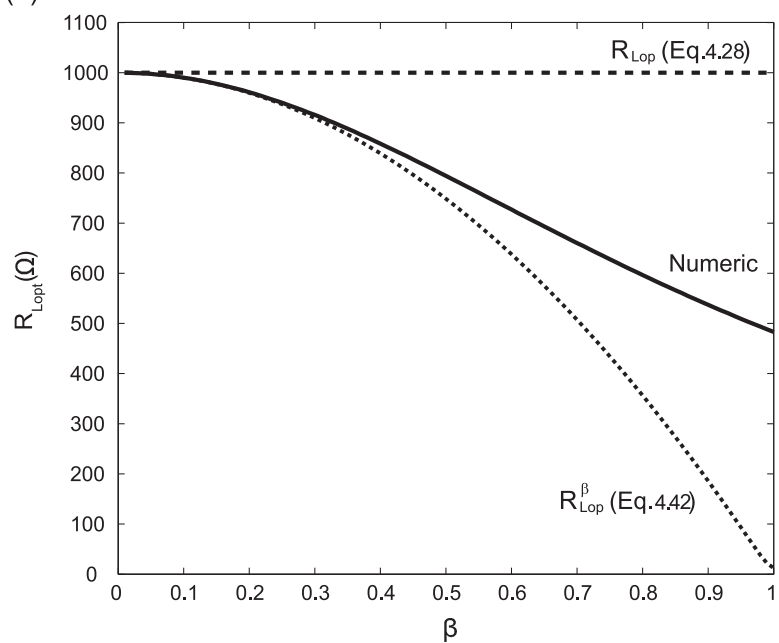

(b)

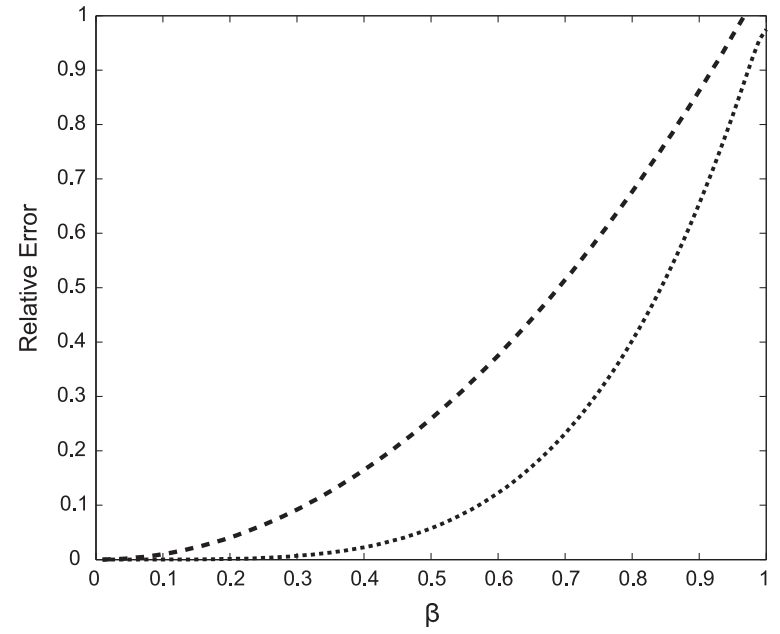

Figure 4.8: (a) Optimal electrical load resistance variation with $\beta$ computed numerically (solid line) and given by analytic approximation (Eq. 4.42). It is also shown (dashed line) the optimal value predicted when inductance effects are not considered (Eq. 4.27). (b) Relative error variation with $\beta$ for the analytic approximation when inductance effects are not considered (dashed line) and for the analytic approximation when inductance effects are considered (dotted line). $U^{*}=14.94$ and parameters listed in Table 4.2.

this result with numerical resolution of the complete system in order to quantify the validity of the assumptions made. To this end, an optimization method was employed to maximize the efficiency given by Eq. 4.17 together with a a fourth order Runge-Kutta scheme to numerically solve Eqs. 4.14a and $4.14 \mathrm{~b}$. It has been considered the same parameters that were presented earlier in a practical example (Table 4.3.2.1.3) and $U^{*}$ was fixed at a value of 14.93 (note that the optimal $R_{L}$ predicted was 1000 Ohms in that case for $\beta=0$, see Fig. 4.7a), and $\beta$ was varied between 0 and 1 . 
As it can be seen from Fig. 4.8a the $R_{\text {Lopt }}^{\beta=0}$ given for the simplified analysis where $\beta$ was neglected (dashed line) is a good approach up to values of beta around 0.1 where deviations between $R_{L o p t}^{\beta=0}$ and the numeric computed value (solid line) is around one per cent. Note that such approximation leads to values of the electrical load higher than the required ones. The analytic prediction for $R_{\text {Lopt }}^{\beta}$ where the inductance effect is considered by a second order perturbation approach (Eq. 4.42), dotted line, is valid until $\beta$ values of 0.3 with deviations lower than one per cent with respect to the numerical computed value. As a comparison, for $\beta=0.3$ the relative error considering $R_{L o p t}^{\beta=0}$ would be around

10 per cent. For completeness, Fig. $4.8 \mathrm{~b}$ shows the relative error for $R_{\text {Lopt }}^{\beta=0}$ and $R_{\text {Lopt }}^{\beta=0}$ as a function of $\beta$. As it can be seen, $R_{L o p t}^{\beta}$ is a valid approximation for $\beta$ values up to 0.6 (relative error less that 10 per cent).

\subsubsection{Concluding remarks}

An electro-fluid-elastic model has been presented and developed to describe in a simplified way the electromagnetic energy harvesting from a flowing fluid taking advantage of transverse galloping oscillations. Two different levels of simplification have been made depending on the comparison between the characteristic electrical and mechanical timescales. In both cases, the effect of the electrical resistance load applied at the electromagnet on the energy harvested has been discussed analytically.

When the characteristic mechanical timescale is very large compared with the characteristic electrical time the inductance of the electromagnet can be neglected and the dynamical effect of the electromagnet in the galloping prism is just to introduce a damping force term. When the characteristic timescales are not so different the inductance needs to be considered since it introduces an additional stiffness term as well as a negative damping term into the dynamics on the galloping prism.

For fixed geometry and mechanical parameters, it has been found that there exists an optimal electrical resistance load for each fluid velocity of the incoming flow. When the optimal electrical resistance load is optimally fixed at each reduced velocity, the efficiency dependence with the reduced velocity is weakened in the sense that once the high level of efficiency is achieved it remains high over a large range of reduced velocities (its behaviour is much more broadband than the case of a fixed value of the electrical resistance load). This is a remarkable result from the practical side and can be helpful to design control laws that maximize energy harvesting for changing flow velocity conditions. The importance of this is further highlighted by considering that it seems easier to tune the load impedance than physically alter the stiffness of the elastic support of the galloping prism. Noteworthy, the efficiency of energy extraction through an electromagnetic generator is equivalent to the maximum achievable given by the cross-section $\left(\eta_{F}\right)$ modulated by factors associated to losses in parasitic damping and losses by resistances in the coil. In addition, with respect to the optimal electrical load resistance, it has been shown that the inductance effects (that is, $\beta$ ) makes its value lower than for the case where $\beta=0$. 


\subsection{Optimal piezoelectric energy extraction}

Nowadays, ambient vibration based energy harvesting devices are being considered by both the scientific community and industry for their potential application to the power supply of small electronic components, like Micro-Electro-Mechanical Systems (Stephen, 2006) or Wireless Networks (Park et al., 2008). The aim is to reduce the dependency of these devices on small batteries, which usually have a limited lifetime and often need maintenance. A promising concept to produce small quantities of electrical power has been proposed very recently (Erkturk (2009), Sirohi and Mahadik (2011,2012), Zhao et al. (2013), Yang et al., 2013). It considers a rigid elongated prism, prone to suffer galloping (i.e. with the appropriate cross-section shape), attached to the free end of a cantilevered beam that has electricity conversion piezoelectric sheets fixed to its base. The strain induced in the beam when the galloping prism is put into oscillatory motion by an airstream is converted into electrical current by the piezoelectric sheets. This electrical current is dissipated at a generical electrical load resistance (see Fig. 4.9 for illustration). This arrangement, where piezoelectric conversion is used, has some practical advantages over electromagnetic and electrostatic means because of its low cost, low maintenance and straightforward mounting. The potential of this energy harvester justifies the development of a theoretical model to analyze in detail the influence of the main governing parameters, namely the cross-section geometry, mechanical properties, electrical properties of the piezoelectric, electrical load, and air speed on the electric generation.

A coupled electro-aero-elastic model for piezoelectric energy harvesting from transverse galloping is thus developed here. The model considers a one degree-of-freedom galloping oscillator where aerodynamic forces are described resorting to quasi-steady conditions. The piezoelectric generator is modeled by an equivalent electrical circuit where power is dissipated at an electrical load resistance. The mathematical model predicts the influence of the main governing parameters: cross-section geometry, mechanical properties, electrical properties of the piezoelectric generator, electrical load, and velocity of the incoming airstream on the electric generation. As it will be shown, the analysis provides a theoretical basis to assess the suitability of the prism geometry and to define the mechanical and piezoelectrical characteristics that maximize the electrical output. In addition, it has been found that there exists an optimal electrical resistance load that maximizes the energy harvested for each velocity of the flow. All these results can be helpful to better understand existing experimental results, to carry out new experiments in order to explore the role of an specific parameter on the energy extraction while maintaining other dimensionless parameters fixed, or when designing piezoelectric energy devices based on transverse galloping. The model is also adapted to the practical situation in which the galloping oscillator is a cantilevered beam. Because of the engineering interest of this particular case, the model results are compared to some experimental results obtained from specialized literature. In view of this comparison, a discussion on the advantages and shortcomings of the proposed model is presented.

Results in this Section 4.4 have been sent to publication to Journal of Wind Engineering \& Industrial Aerodynamics.

\subsubsection{Mathematical model}

The device under consideration is shown schematically in Fig. 4.9. It consists of a cantilever beam where piezoelectric patches are fixed. A rigid prism (whose section is prone to galloping) is attached to the free end of the cantilever beam. Under the effect of an airstream, for high enough air speed, oscillations by transverse galloping take place in such a way that the induced strain in the piezoelectric patches produces an electrical current which is dissipated at the electrical load resistance $R_{L}$. When the length of the beam is large compared to the prism cross-section length, the motion of the prism can be considered nearly transversal to the incident flow and a transverse galloping phenomenon occurs. 
For the effect of cantilevered beams on the dynamics of transverse galloping see Kluger et al. (2013), $\mathrm{Xu}-\mathrm{Xu}$ et al. (2016b) as well as in Appendix I, where corrections due to the effect of cantilevered beam to the dynamical response are presented. Note that motion occurs also along the in-line flow direction.

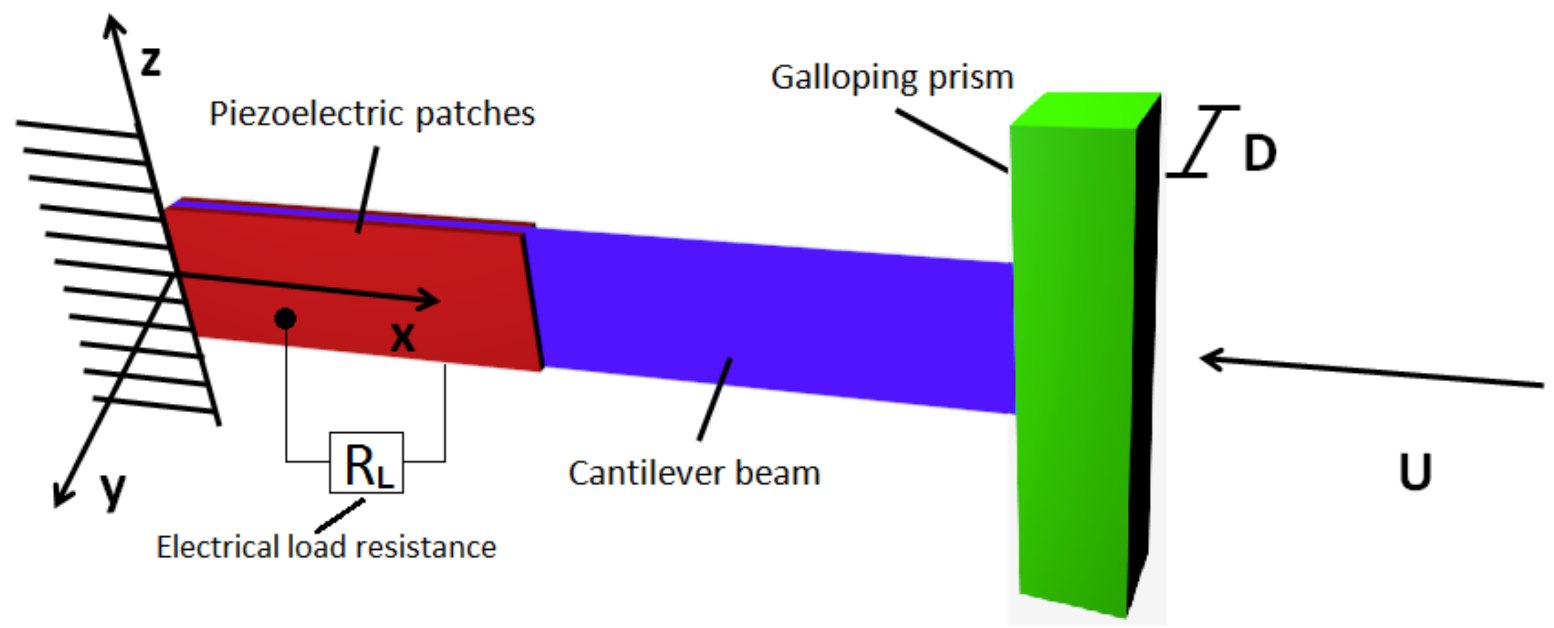

Figure 4.9: Schematic of a galloping prism prone to galloping transverse to the incident airflow.

\subsubsection{Piezoelectric Model}

(a)

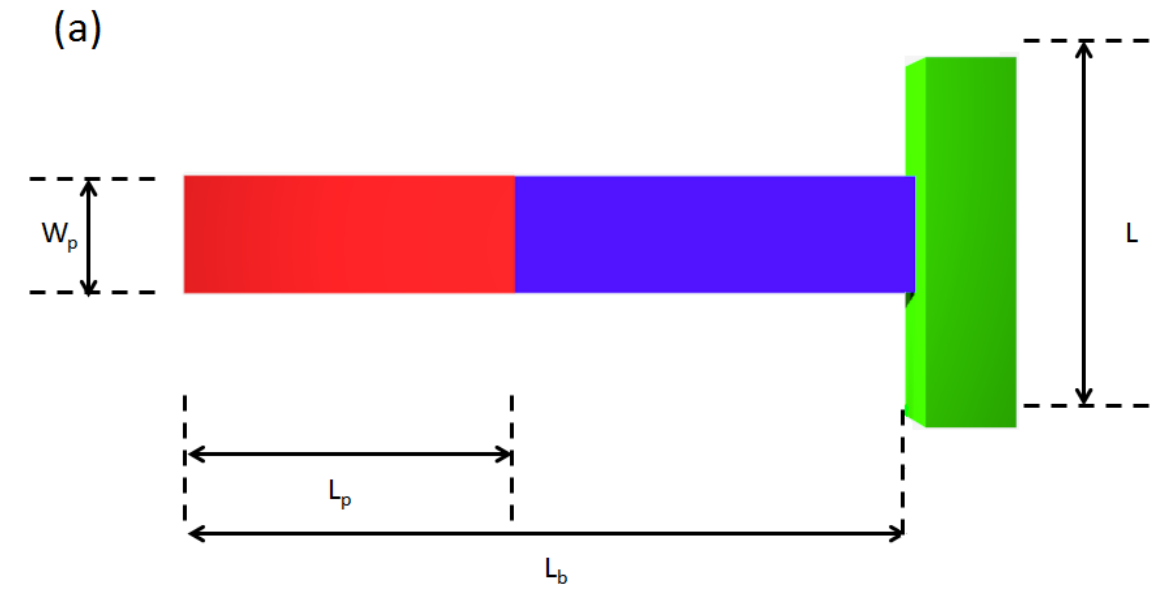

(b)

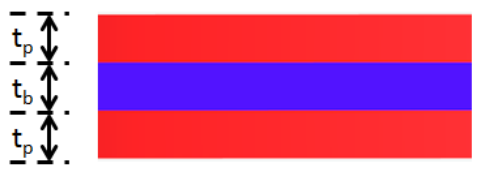

Figure 4.10: (a) Side view of the galloping prism attached to a cantilever beam with piezoelectric patches. (b) Detail of the thickness of the triple-layer piezoelectric mounted on the cantilever beam.

To describe the backlash electromechanical force caused by the effect of the piezoelectric sheets, let us consider a lumped model such as the one developed and tested in references Erkturk (2009), Shu and Lien (2006), Akaydin et al. (2012), Afsharfard et al. (2014) or Wang and Lu (2014). This model considers that a triple-layer bender is mounted on a cantilever beam with polarization poled along the thickness direction, (Fig. 4.10) where the electric field is generated through the thickness of the piezoelectric layers. The cantilever beam has a length $L_{b}$ whereas the piezoelectric sheets which 
are situated on the base have an axial length of $L_{p}$ with the only condition that $L_{p} \leq L_{b}(L$ is the length of the prism). The mathematical model that describes the induced electromechanical backlash force $F_{T}$, per unit length of the prism, and provides the relationship between the main parameters of the piezoelectric sheet and the electrical load is:

$$
\begin{gathered}
C_{p} \frac{d v}{d t}+\frac{v}{R_{L}}+\theta \dot{y}=0, \\
F_{T}=-\frac{\theta v}{L},
\end{gathered}
$$

where $v$ is the voltage across the resistive load $R_{L}$ connected to the piezoelectric sheets. Furthermore, $C_{p}$ is the equivalent capacitance of the piezoelectric layers and $\theta$ is an electomechanical coupling constant which can be easily determined through experiments by measuring the open circuit and short circuit resonant frequencies of the cantilever beam as explained in Zhao et al. (2013). Furthermore, by applying a modal analysis, coefficients $C_{p}$ and $\theta$ can be analytically determined

$$
\begin{gathered}
C_{p}=\kappa_{C_{p}} \frac{W_{p} L_{p}}{t_{p}} \epsilon_{33}^{s}, \\
\theta=\left.\kappa_{\theta} \frac{d \phi}{d x}\right|_{x=L_{p}} W_{p}\left(t_{b}+t_{p}\right) e_{31},
\end{gathered}
$$

in which $e_{31}$ is the piezoelectric stress coefficient and $\epsilon_{33}^{s}$ is the permittivity component at constant strain, $W_{p}$ is the width of the piezoelectric patches, $d \phi /\left.d x\right|_{x=L_{p}}$ is the curvature of the piezoelectric sheets at their end and $t_{p}$ and $t_{b}$ are the thicknesses of the piezoelectric sheets and of the beam respectively. The parameters $\kappa_{C_{p}}$ and $\kappa_{\theta}$ depend on whether the connection between the two piezoelectric layers has been done in series $\left(\kappa_{C_{p}}=1 / 2, \kappa_{\theta}=1\right)$ or in parallel $\left(\kappa_{C_{p}}=2, \kappa_{\theta}=2\right)$ such as presented in Erkturk (2009).

The dynamical equations that model the galloping phenomenon with backlash force $F_{T}$ from the piezoelectric sheets problem (Eqs. 4.2 and 4.43) can be rewritten non-dimensionally with $V=v / V_{c}$ being $V_{c}=m \omega_{N}^{2} D L / \theta$ as

$$
\begin{gathered}
Y^{\prime \prime}+2 \zeta Y^{\prime}+Y=\frac{U^{* 2}}{2 m^{*}}\left(a_{1} \frac{Y^{\prime}}{U^{*}}+a_{3}\left(\frac{Y^{\prime}}{U^{*}}\right)^{3}\right)+V \\
\beta V^{\prime}+V+2 k_{E} \beta Y^{\prime}=0
\end{gathered}
$$

where $\beta=R_{L} C_{p} \omega_{N}$ is the relationship between the characteristic electrical and mechanical times and $k_{E}=\theta^{2} /\left(2 m L C_{p} \omega_{N}^{2}\right)$ is a non-dimensional parameter that refers to the energy production capacity of the piezoelectric sheets for a given prescribed oscillation frequency.

In this case, the power of energy extraction through the piezoelectric sheets can be described as

$$
P_{E}=\frac{1}{T} \int_{0}^{T} \frac{v^{2}}{R_{L}} d t
$$

$T$ being an averaging time. The efficiency of energy extraction through the piezoelectric sheets $\left(\eta_{E}\right)$ is defined equivalently as in Eq. 4.17, $\eta_{E}=2 P_{E} / \rho U^{3} D L$.

The system given by Eqs. 4.45 and 4.46 allows to obtain the galloping response and the power dissipated at $R_{L}$ as a function of the mechanical parameters $\left(m^{*}, \zeta, \omega_{N}\right)$, geometrical and flow parameters $\left(U^{*}, a_{1}, a_{3}, D\right)$, and parameters of the piezoelectric generator $\left(k_{E}, \beta\right)$. 


\subsubsection{Galloping response and energy efficiency}

As aforementioned, let us try to achieve an analytical approximation of Eqs. 4.45a and 4.45b which can be solved approximately when the nonlinear terms are small compared to the linear ones $\left(m^{*} U^{*} \gg 1\right)$, which typically happens when the fluid in consideration is air. In this case, one may assume that the steady state motion is sinusoidal, that is: $Y=A^{*} \sin \left(\omega^{*} \tau\right)$ and $V=V \cos \left(\omega^{*} \tau+\varphi\right)$, where $\omega^{*}=\omega / \omega_{N}, A^{*}, V$, and $\varphi$ have to be solved. Substituting this into Eq. 4.45a and taking into account that $\left(A^{*} \omega^{*} \sin \left(\omega^{*} \tau\right)\right)^{3} \approx 3 A^{* 3} \omega^{* 3} \sin \left(\omega^{*} \tau\right) / 4$, and after some algebra one gets

$$
\begin{gathered}
2 \zeta A \omega^{*}=\frac{U^{* 2}}{2 m^{*}}\left(a_{1} \frac{A \omega^{*}}{U^{*}}+\frac{3}{4} a_{3} \frac{A^{3} \omega^{* 3}}{U^{* 3}}\right)+V \cos \varphi, \\
A\left(1-\omega^{* 2}\right)=-V \sin \varphi
\end{gathered}
$$

Equating sine and cosine terms from Eq. $4.45 \mathrm{~b}$, it yields

$$
\begin{gathered}
-\beta \omega^{*} V \cos \varphi-V \sin \varphi=0 \\
-\beta \omega^{*} V \sin \varphi+V \cos \varphi+2 k_{E} \beta A \omega^{*}=0 .
\end{gathered}
$$

The non-dimensional galloping oscillation frequency can be obtained by solving Eqs. 4.47b and 4.48a, yielding

$$
\omega^{* 2}=\frac{-\left(1-\beta^{2}-2 k_{E} \beta^{2}\right)+\sqrt{\left(1-\beta^{2}-2 k_{E} \beta^{2}\right)^{2}+4 \beta^{2}}}{2 \beta^{2}},
$$

This means that within the assumed hypothesis (the quasi-steady motion and small nonlinearities), the non-dimensional oscillating frequency of the system $\omega^{*}$ depends on the piezoelectric parameters $k_{E}$ and $\beta$ only as fluid forces are in phase with the velocity terms. Furthermore, when $k_{E}$ is very small (which is typical for small commercial piezoelectric energy sheets, for example, from Table 4.3, experiments carried out by Zhao et al. (2013), yield a value of $k_{E} \simeq 6.2 \cdot 10^{-3}$ ), the oscillating frequency of the prism is close to one for all values of $\beta$, and therefore the oscillating frequency may be approximated by

$$
\omega^{* 2}=1+\frac{2 \beta^{2}}{1+\beta^{2}} k_{E},
$$

and thus, the variation of the frequency is very small for all values of $\beta$.

The phase difference between the oscillation of the galloping prism and the voltage of the electric circuit due to the piezoelectric beam is derived from Eq. $4.47 \mathrm{~b}$

$$
\varphi=\tan ^{-1}\left(-\beta \omega^{*}\right)
$$

The ratio between the non-dimensional voltage and the non-dimensional amplitude of oscillation of the prism is (as obtained from Eq. 4.48b)

$$
\frac{V}{A^{*}}=\frac{2 k_{E} \beta \omega^{*}}{\beta \omega^{*} \sin \varphi-\cos \varphi} .
$$

Finally, the non-dimensional amplitude of the prism can be solved from Eq. 4.47b

$$
A^{* 2}=\frac{4 U^{*}}{3 a_{3} \omega^{* 2}}\left(4 m^{*}\left(\zeta+\frac{k_{E} \beta}{1+\beta^{2} \omega^{* 2}}\right)-a_{1} U^{*}\right) .
$$

A piezoelectric equivalent damping $\left(\zeta_{p}=k_{E} \beta /\left(1+\beta^{2} \omega^{* 2}\right)\right)$ can be defined, which depends on the piezoelectric sheets $\left(k_{E}\right)$, the non-dimensional load resistance $(\beta)$ and the reduced frequency of oscil- 
lation $\left(\omega^{*}\right)$. It is important to note that an initial galloping velocity can be defined from Eq. 4.53. $A^{*}$ has physical meaning $\left(A^{*}>0\right)$ when the reduced velocity exceeds a critical value $U_{g}^{*}$, which is the reduced velocity at which the system starts galloping, meaning that the destabilizing effect of the fluid force equals the stabilizing effect of both the mechanical damping and the piezoelectric equivalent damping.

$$
U_{g}^{*}=\frac{4 m^{*}}{a_{1}}\left(\zeta+\zeta_{p}\right)
$$

Note the dependence with $\beta$ (via $\zeta_{p}$ ), and thus the galloping velocity $U_{g}^{*}$ depends on the electrical load chosen $R_{L}(\beta)$. From Eq. 4.54, and assuming $\omega^{*} \simeq 1$, it follows

$$
\frac{\partial U_{g}^{*}}{\partial \beta} \propto \frac{1}{\beta^{2}+1}-\frac{2 \beta^{2}}{\left(\beta^{2}+1\right)^{2}},
$$

which means that $\partial U_{g}^{*} / \partial \beta>0$ for $\beta<1, \partial U_{g}^{*} / \partial \beta=0$ for $\beta=1$ and $\partial U_{g}^{*} / \beta<0$ for $\beta<1$. This results agrees with numerical simulations given in Abdelkefi et al. (2013c) and experimental results in Zhao et al. (2013).

Once the solution for the dynamical response of the system has been derived, from Eq. 4.46 the power extracted through the galloping phenomenon of the piezoelectric beam in the electrical load is

$$
P_{E}=\frac{1}{T} \int_{0}^{T} \frac{v^{2}}{R_{L}}=\frac{2 V_{c}^{2}}{R_{L}} \frac{k_{E}^{2} \beta^{2}}{1+\omega^{*} 2 \beta^{2}} A^{* 2} \omega^{* 2} .
$$

With regard to the efficiency of energy extraction $\eta_{E}$,

$$
\eta_{E}=\frac{8 m^{*} k_{E} \beta}{\left(1+\beta^{2} \omega^{* 2}\right) 3 a_{3} U^{* 2}}\left(4 m^{*}\left(\zeta+\zeta_{p}\right)-a_{1} U^{*}\right),
$$

which states the dependence of the efficiency with the geometry of the cross-section of the prism $\left(a_{1}\right.$, $\left.a_{3}\right)$, mechanical parameters $\left(m^{*}, \zeta\right)$, piezoelectric parameters $\left(k_{E}, \beta, \zeta_{p}\right)$, and flow speed $\left(U^{*}\right)$.

\subsubsection{Role of $U^{*}$ and $\beta$ on the efficiency of the piezoelectric power har- vester.}

In this section, the efficiency of the energy harvesting as defined by Eq. 4.57 is discussed. From the practical side, it is of interest to design a control law for $R_{L}$ so as to extract the maximum power even in varying flow speed $\left(U^{*}\right)$. To better discuss this question, the efficiency dependence with the electrical load and the reduced velocity will be studied keeping the other variables constant.

\subsubsection{Maximum efficiency achievable for fixed mechanical/piezoelectric configuration}

In first place, let us consider $\beta$ to be constant and fixed, then, the maximum value of the efficiency is achieved when $\partial \eta / \partial U^{*}=0$. By solving the previous equation, it is obtained that the maximum efficiency for constant $\beta$ always happens at twice the initial galloping velocity $U_{g}^{*}$

$$
U_{\eta_{E}^{\max }}^{*}=2 U_{g}^{*}=\frac{8 m^{*}\left(\zeta+\zeta_{p}\right)}{a_{1}}
$$

Substituting the value of the reduced velocity at which the maximum efficiency happens (Eq. 4.58) into Eq. 4.57, yields that the maximum efficiency for each fixed $\beta$ is

$$
\eta_{E}^{\max }=-\frac{a_{1}^{2}}{6 a_{3}}\left(\frac{1}{1+\zeta / \zeta_{p}}\right)=\eta_{F}\left(\frac{1}{1+\zeta / \zeta_{p}}\right)
$$


which relates the maximum achievable efficiency with the cross-section geometry of the prism $\left(a_{1}\right.$ and $a_{3}$ ), namely $\eta_{F}$ as in 4.12 and 4.25 , and the ratio between the mechanical damping and the piezoelectric equivalent damping $\zeta / \zeta_{p}$ (which accounts for losses as parasitic damping).

\subsubsection{Optimal electrical load for each $U^{*}$}

From the practical side, as already anticipated, it would be desirable to design a control law for the electrical resistance load in which the power harnessed at each velocity of the incident would be maximized. Such control law can be directly obtained by setting $\partial \eta / \partial \beta=0$. Solution of the previous equation yields the optimal value of $\beta$ (i.e. the load resistance) that maximizes the power harnessed for a given reduced flow velocity $U^{*}$, which is convenient when dealing with actual flows in which the reduced velocity changes. This concept is similar to a tracking-point strategy for variable flow conditions.

An analytical solution of the $\partial \eta / \partial \beta=0$ condition requires a further level of simplification for the frequency oscillation (Eq. 4.49) since it depends on the electrical load resistance, by considering $\omega^{*}=1$ for all values of $\beta$. Such simplification is reasonable as the variation of the frequency for short circuit $(\beta=0)$ and open circuit $(\beta=\infty)$ is very small for typical applications of piezoelectric patches (as discussed in Eq. 4.50). Using such simplification, the values of the optima for $\beta$ as a function of $U^{*}$ may be obtained

$$
\begin{gathered}
\beta_{o p 1}=\frac{4 m^{*} k_{E}-\left(-U^{* 2} a_{1}^{2}+8 U^{*} a_{1} m^{*} \zeta+16 k_{E}^{2} m^{* 2}-16 m^{* 2} \zeta^{2}\right)^{1 / 2}}{U^{*} a_{1}-4 m^{*} \zeta}, \\
\beta_{o p 2}=\frac{4 m^{*} k_{E}+\left(-U^{* 2} a_{1}^{2}+8 U^{*} a_{1} m^{*} \zeta+16 k_{E}^{2} m^{* 2}-16 m^{* 2} \zeta^{2}\right)^{1 / 2}}{U^{*} a_{1}-4 m^{*} \zeta} \\
\beta_{o p 3}=1 .
\end{gathered}
$$

It can be proven that $\beta_{o p 1}$ and $\beta_{o p 2}$ only exist for reduced velocities smaller than a characteristic one we shall call $U_{\text {joint }}^{*}\left(U^{*}<U_{\text {joint }}^{*}\right)$ and that they are local maxima; $\beta_{o p 3}$ is a local minimum for $U^{*}<U_{\text {joint }}^{*}$ and a local maximum for $U^{*}>U_{\text {joint }}^{*}$. The value of $U_{\text {joint }}^{*}$ can be obtained by equating $\beta_{o p 1}$ and $\beta_{o p 2}$. By doing so,

$$
U_{\text {joint }}^{*}=\frac{4 m^{*}\left(\zeta+k_{E}\right)}{a_{1}}
$$

Eq. 4.60 represents an analytical expression for the control law which maximizes the power harnessed and broadens the efficiency curve over a range of velocities. In the following sections we will return to this questions with specific examples.

\subsubsection{Global maximum efficiency}

Finally, we would like to determine the values of $\beta$ and $U^{*}$ for which the global maximum efficiency is obtained. This takes place when both $\partial \eta / \partial U^{*}=0$ and $\partial \eta / \partial \beta=0$. The values of the electrical load and the reduced incident velocity that fulfill both equations are

$$
\begin{gathered}
\beta_{\max }^{G}=1, \\
U_{\max }^{* G}=\frac{4 m^{*}\left(2 \zeta+k_{E}\right)}{a_{1}},
\end{gathered}
$$

which yields a maximum global efficiency of

$$
\eta_{E}^{G, \max }=\frac{-a_{1}^{2}}{6 a_{3}} \frac{1}{1+2 \zeta / k_{E}} .
$$


Note that superscript $G$ stands for "global". It is interesting to note that the difference in reduced velocity between $U_{\max }^{* G}$ and $U_{\text {joint }}^{*}$ is

$$
\Delta U^{*}=\frac{4 m^{*} \zeta}{a_{1}}
$$

which becomes smaller as $m^{*} \zeta$ diminishes and $a_{1}$ increases.

Practical consequences may be derived from Eq. 4.64. We should design our energy harvester depending on the site and the velocity of the incoming flow to adjust the reduced velocity so as to be at $U^{*}=U_{\max }^{* G}$ as a design point. In order to be at such point, given a physical incoming flow velocity, we will have to correctly choose $\omega_{N}, D, m^{*}, \zeta, k_{E}$, or $a_{1}$. If $U^{*}$ changes from its nominal value (typical behavior when involving actual flows), $\beta$, and therefore $R_{L}$, will have to be modified accordingly using Eqs. 4.60 so as to be at the maximum possible efficiency for each $U^{*}$. Another important issue is related with the global maximum efficiency that can be achieved. From Eq. 4.64, we can divide the efficiency into two factors

$$
\eta_{\max }^{G}=\eta_{\mathrm{F}} \eta_{\text {piezo }}=\frac{-a_{1}^{2}}{6 a_{3}} \frac{1}{1+2 \zeta / k_{E}}
$$

where $\eta_{\mathrm{F}}=-a_{1}^{2} /\left(6 a_{3}\right)$ only depends on the $C_{y}$ dependence with the angle of attack $\alpha_{F}$, which in turn depends on the cross-section shape of the prism mainly, and $\eta_{\text {piezo }}$ compares the energy dissipated at the electrical load connected to the piezoelectric sheets with the losses as parasitic damping.

It is worth mentioning that the best cross-sections are those that have large values of $a_{1}$ and small values of $a_{3}$ (in absolute terms) (see Table 4.1). On the other hand, to obtain acceptable values of $\eta_{\text {piezo }}$, which relates the energy harvested to the power lost as parasitic damping, it is essential to obtain high values of $k_{E} / \zeta$ (see Eq. 4.66). The non-dimensional parameter $k_{E}$ relates the capacity of the piezoelectric sheets of producing energy for a given prescribed velocity of oscillation and $\zeta$ is the non-dimensional damping coefficient associated to mechanical dissipation. Additionally, it may be rewritten as

$$
\frac{k_{E}}{\zeta}=\frac{\theta^{2}}{C_{p} c L \omega_{N}}=\frac{\kappa_{\theta}^{2}}{\kappa_{C_{p}}} \frac{\phi^{\prime}\left(L_{p}\right)^{2}}{L_{p}} \frac{e_{31}^{2}}{\epsilon_{33}^{s}} \frac{W_{p}\left(t_{b}+t_{p}\right)^{2} t_{p}}{c L \omega_{N}},
$$

where $\phi^{\prime}\left(L_{p}\right)=\left.(d \phi / d x)\right|_{x=L_{p}}$. In this equation, different parameters are related and should be designed in such a way to maximize $k_{E} / \zeta$. The effect of the electrical connectivity, which for the study case of two piezoelectric sheets can be done in series or in parallel, is recovered in the parameter $k_{\theta}^{2} / k_{C_{P}}$ which takes a constant value of 2 for both types of connection and thus the optimization of $\eta_{\text {piezo }}$ does not depend on the type of connection used for the piezoelectric sheets. Concerning the strain distribution on the piezoelectric sheet, an interesting question is related to the length of the piezoelectric sheets $\left(L_{p}\right)$. As a first approximation, if it can be considered that the effect of the piezoelectric sheets on the stiffness of the cantilever beam and on its deformation mode is negligible, then the optimal length of the piezoelectric sheets, can be obtained by differentiating $\phi^{\prime}\left(L_{p}\right)^{2} / L_{p}$ and equating to zero. Taking an Euler-Bernoulli approximation, it is seen by modal analysis that the first mode of deformation of the beam is given by $\phi(x)=1 / 2\left\{\cosh \left(\beta_{1} x\right)-\cos \left(\beta_{1} x\right)-k_{1}\left(\sinh \left(\beta_{1} x\right)-\sin \left(\beta_{1} x\right)\right)\right\}$ where $k_{1}=0.7341$, $\beta_{1}=1.8751 / L_{b}$ and $x$ is the axial coordinate Arfsharfard and Farshidianfar (2014). Thus, by taking into account the condition of maximum, the optimal length for the piezoelectric sheets is found to be $L_{p}^{o p}=0.51 L_{b}$. This value emerges as a compromise between maximizing the curvature and minimizing the length of the sheets and thus the length of the piezoelectric sheets should be chosen accordingly. Nevertheless, note that other possibilities are also possible to enhance $\phi^{\prime}\left(L_{p}\right)$. For example, in Zhao and Yang (2015) a beam stiffener has been proposed to magnify the slope of the mode shape at the end of the piezoelectric sheets $\left(x=L_{p}\right)$. 


\begin{tabular}{cccccccccc}
\hline Variable & Value & \multicolumn{10}{c}{} \\
\hline$m$ & $k$ & $a_{1}$ & $a_{3}$ & $\mathrm{D}$ & $\mathrm{L}$ & $\rho$ & $\zeta$ & $C_{p}$ & $\theta$ \\
$0.207 \mathrm{~kg} / \mathrm{m}$ & $381.6 \mathrm{~N} / \mathrm{m}$ & 2.3 & -18 & $0.04 \mathrm{~m}$ & $0.15 \mathrm{~m}$ & $1.2 \mathrm{~kg} / \mathrm{m}^{3}$ & 0.005 & $180 \mathrm{nF}$ & $3.73 \cdot 10^{-4} \mathrm{~N} / \mathrm{V}$ \\
\hline
\end{tabular}

Table 4.3: Physical properties considered in the example for the galloping body and the piezoelectric beam as obtained from Zhao et al. (2013). Values of the coefficients $a_{1}$ and $a_{3}$ are taken from Table 4.1 for the square section.

\subsubsection{Model validation with experimental results}

Experiments found in the literature typically consider short cantilevered beams in which the dynamics differ from the pure transverse galloping and thus, for the sake of completeness, the effect of the cantilevered beam on the dynamic response should be included in the analysis. When it cannot be assumed that the length of the cantilever beam is much longer than the diameter of the galloping prism, a correction has to be implemented into the dynamics equation. The analytical treatment for short cantilever beams is done in Appendix A, where a correction to the oscillation frequency and oscillation amplitude is obtained.

A comprehensive set of experiments of transverse galloping based piezoelectric energy harvesting has been published in Zhao et al. (2013). Table 4.3 lists the mechanical and piezoelectric parameters in their experiments. It is noteworthy that $L_{b} / D=3.75$ and thus, the effect of the cantilever beam cannot be neglected, and so, correcting factors (as derived in Appendix A) are needed in the model to predict the power extracted for each configuration.

In Fig. 4.11, a comparison between experimental results and analytical results (Appendix A) for the power extraction is made for different incident velocities ( $U=4,5$ and $6 \mathrm{~m} / \mathrm{s}$ respectively). In Fig. 4.12a, for fixed electric load $R_{L}=105 \mathrm{k} \Omega$, the power output is given as a function of the air velocity. In Fig. 4.12b, the efficiency of the power extraction has been presented (Eq. A.11). The analytical model predicts the efficiency and the location of the maximum (obtained from Fig. A.1 and Eq. 4.54, with a value of the physical incident velocity of $U=3.94 \mathrm{~m} / \mathrm{s}$ ) with good agreement. In Fig. 4.12c the relation between the efficiency from pure transverse galloping and the cantilever one is compared. In dashed line the numerically computed relation is presented whereas the solid one is obtained by considering that $U^{* 2} \delta^{2} / m^{*} \ll 1$ (see Appendix A), and when the condition is fulfilled, the approximation considered is correct. Basically, using short cantilever beams translates into being able to harness less power. Also, the maximum efficiency appears for lower incident velocity of the airflow. Therefore, if possible, when designing a practical application, it is convenient to use long cantilever beams. Analytical results compare well with the experimental tests carried out, validating the theoretical model as long as the hypothesis considered hold.

\subsubsection{Practical design application}

In this section a practical design application is presented for a small portable energy harvester using a long enough cantilever beam similar to the one presented in Fig. 4.9. For this particular case, an isosceles triangle (Alonso et al., 2007) is used as the main galloping prism. From Table 4.1, the coefficients for the transverse force coefficient are $a_{1}=2.9$ and $a_{3}=-6.2$ leading to a maximum achievable efficiency for the given cross-section geometry of $\eta_{F}=0.23$ (see Eq. 4.12).

The device has been designed to work at an airspeed of $9 \mathrm{~m} / \mathrm{s}$ (design point). The energy harvester is made completely in aluminum 6061-T6 except the piezoelectric sheets that are made of P-876.A11 which are commercially available from PI Ceramic GmbH.

With respect to the galloping prism, its cross-section's diameter is $D=0.06 \mathrm{~m}$, being its length $L=0.2$ and mass $M_{\text {prism }}=0.243 \mathrm{~kg}$. The main design parameter variables for the cantilever beam's have been selected as follows. In first place, its length has to be chosen so that the effect of the 

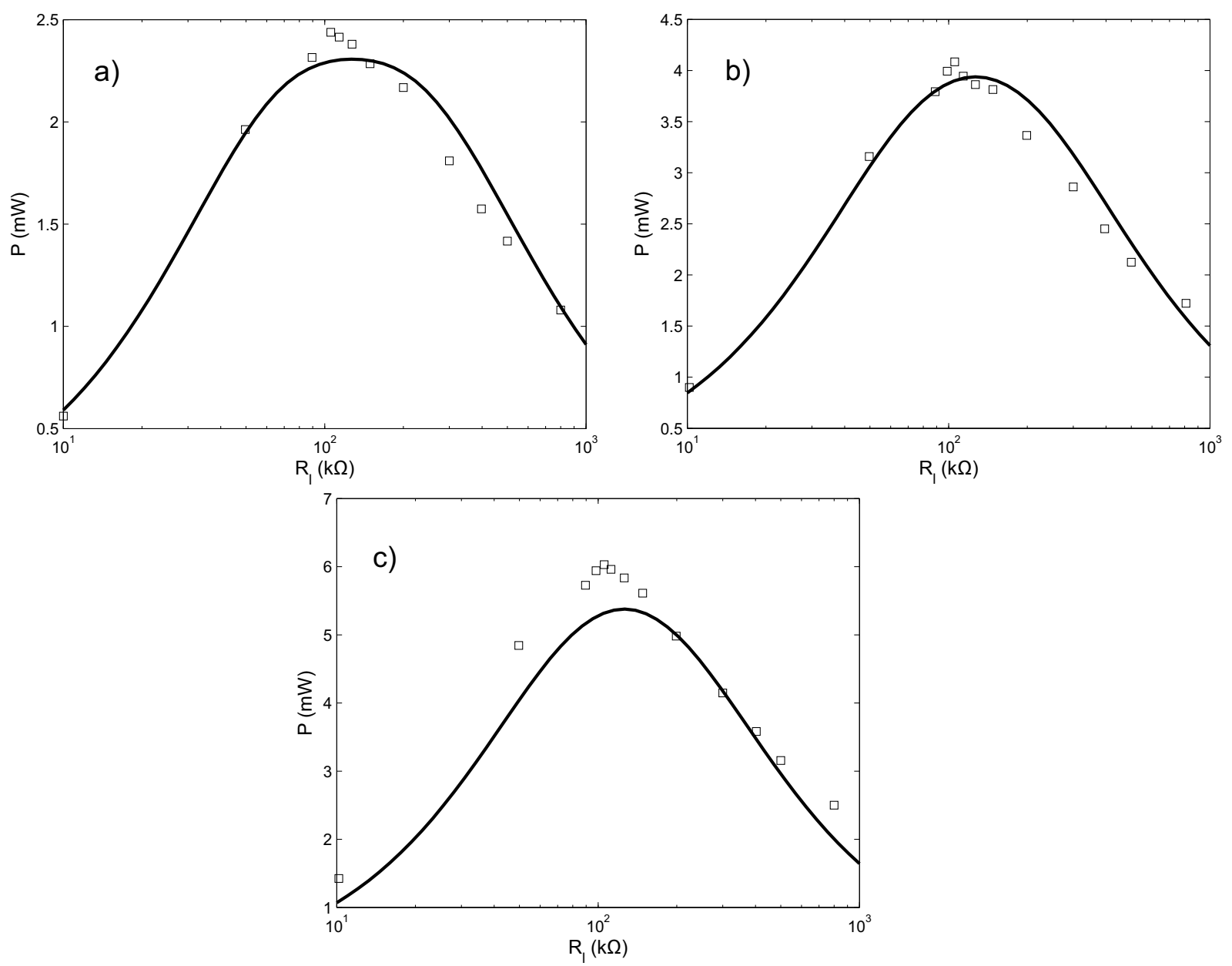

Figure 4.11: Power harnessed for three different incident velocities of the airflow. Open squares correspond to experiments from Zhao et al. (2013), solid lines represent power harnessed taking into account corrections due to the deflection of the cantilever beam's tip.

deflection at the tip is negligible, thus it has been chosen to be $L_{b}=0.6 \mathrm{~m}$. The thickness of the cantilever beam has been chosen to be $t_{b}=0.003 \mathrm{~m}$ and its width $W_{b}=0.08 \mathrm{~m}$. Being Young's modulus $70 \mathrm{GPa}$ for the aluminum selected, the stiffness of the beam is $K=252 \mathrm{~N} / \mathrm{m}$ if the effect of the piezoelectric patches is considered negligible. For the piezoelectric sheets' parameters chosen, it has been used the P-876.A11 from PI Ceramic GmbH as a basis. Its thickness will be $t_{p}=4 \cdot 10^{-4} \mathrm{~m}$ and correspondingly, the width and length of the piezoelectric sheets will be $W_{p}=0.08 \mathrm{~m}$ and $L_{b}=0.3$ $\mathrm{m}$. The piezoelectric sheets mass is $0.037 \mathrm{~kg}$, with a value of $C_{p}=1.37 \cdot 10^{-6} \mathrm{~F}$ and $\theta=9 \cdot 10^{-4}$ $\mathrm{N} / \mathrm{V}$. The mechanical damping of the complete system should be obtained from free oscillation decay experiments of the cantilever in question. However, we propose a typical value of mechanical damping for similar systems from the literature $\mathrm{Xu}-\mathrm{Xu}$ et al. (2015), and thus, $c=4 \cdot 10^{-3} \mathrm{~N} \mathrm{~s} \mathrm{~m}-1$

Fig. 4.13 shows the contour plots for the power's harnessed efficiency as a function of the electric load and the velocity of the air current as obtained from Eq. 4.57. In dashed lines, the optimal electric load $\left(R_{L}\right)$ is plotted as a function of the incident airspeed, indicating the maximum power that can be harnessed at each incident velocity. The solid circle indicates the point at which the maximum efficiency is obtained. The maximum efficiency happens at an incident velocity of $U=9.2 \mathrm{~m} / \mathrm{s}$ for an electric load of $28755 \Omega(\beta=1)$ with a peak efficiency of $\eta_{\text {max }}^{G}=0.17$ (which corresponds to a power of $770 \mathrm{~mW}$ ). As described through Eq. $4.66 \eta_{\text {piezo }}=0.74$ which accounts for losses in the conversion into electric power. Fig. 4.13b, on the other hand, shows the contour plots for the power harnessed 

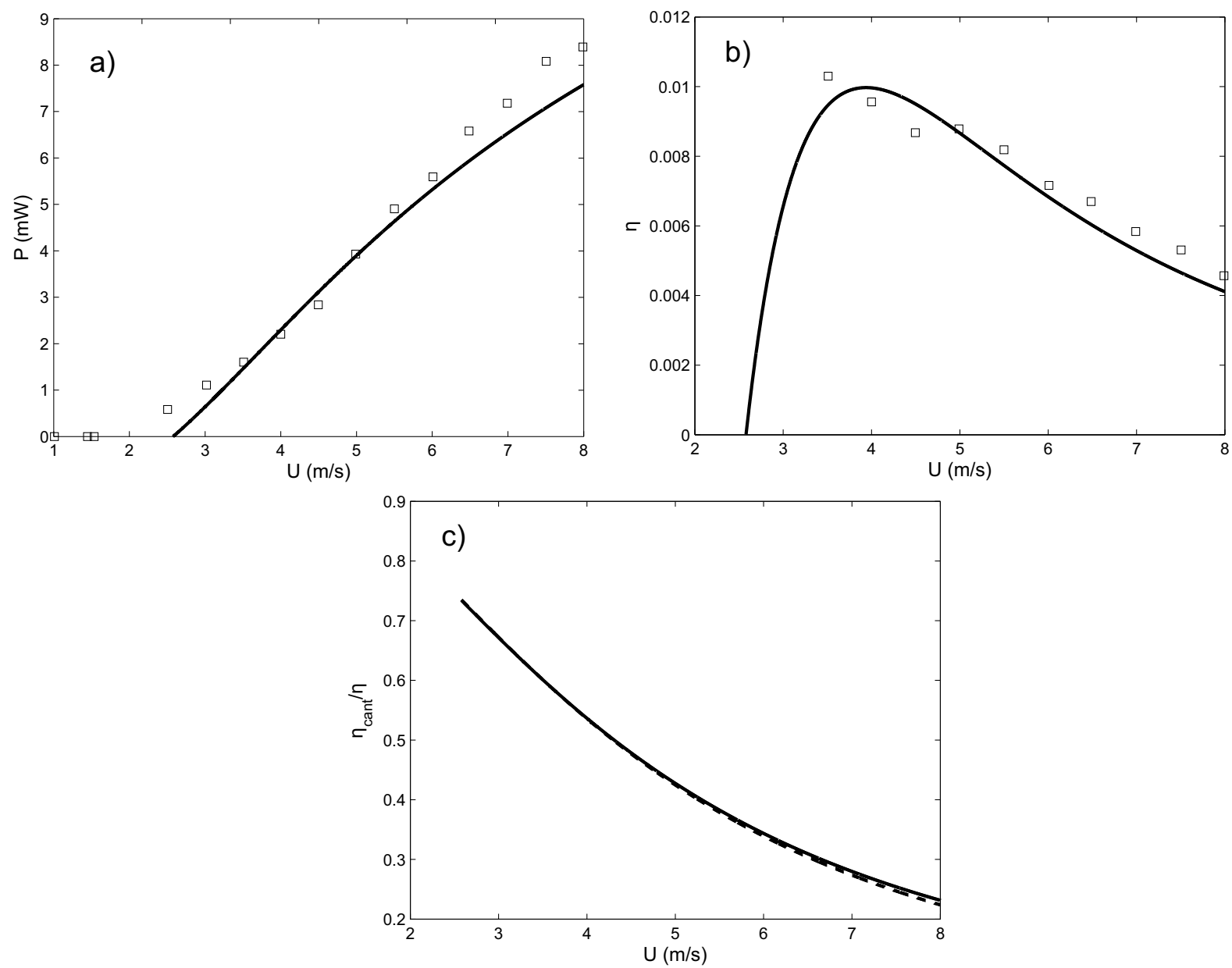

Figure 4.12: (a) Power harnessed for constant electric load $\left(R_{l}=105 \mathrm{k} \Omega\right)$ over a range of incident velocities of the airflow.(b) Energy efficiency conversion for constant electric load. Open squares correspond to experiments from Zhao et al. (2013), solid lines represent power harnessed taking into account corrections due to the deflection of the cantilever beam's tip. (c) Relation between the efficiency of power harnessed taking into account the effect of the deflection at the tip of the cantilever beam with respect to that of pure transverse galloping.

given in miliwatts as a function of the incident velocity and the electric load chosen. The harnessable power increases with the incoming velocity, however, the efficiency at which this extraction occurs diminishes after the incident velocity is larger than a threshold value.

\subsubsection{Concluding remarks}

A theoretical model has been proposed for the coupling between a transverse galloping oscillator and a piezoelectric generator which has been validated with experimental results. This model helps to give some insight in the design process of the energy harvester to maximize the power harnessing efficiency for a given site. In this sense, it is important to select appropriately the shape of the cross-section of the prism as it has straight influence on the amount of energy that can be transferred from the airflow to the harvester (the efficiency of power harvesting). If $a_{1}$ and $a_{3}$ are the linear and cubic coefficients of the polynomial fitting of the aerodynamic force, the efficiency depends on the factor $a_{1} / a_{3}^{3}$. Additionally, $a_{1}$ governs the airstream velocity at which the galloping phenomenon starts, being inversely proportional. If the typical velocity of the airflow is known for a given site, the speed at which the harvester starts to suffer galloping oscillations should be half of the typical velocity of 

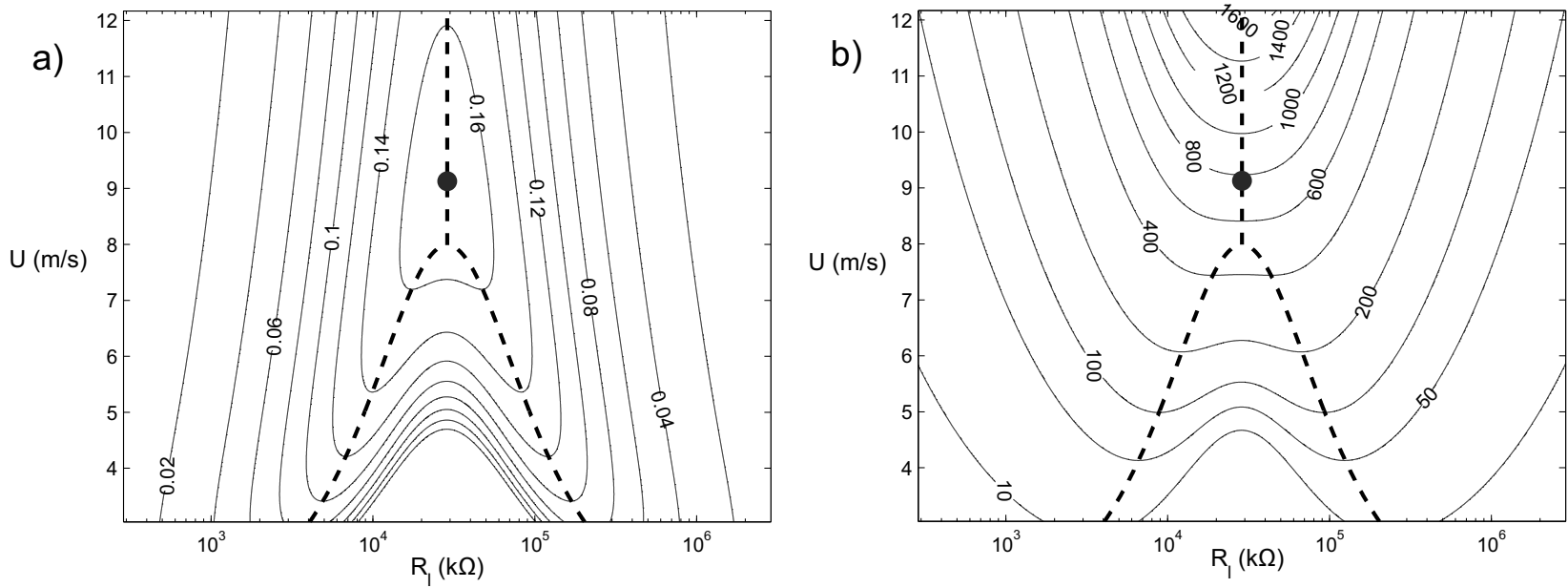

Figure 4.13: (a) Contour map of the efficiency of power harnessed as a function of the incident velocity $U(\mathrm{~m} / \mathrm{s})$ and of the electric load $(\Omega)$. (b) Power harnessed given in miliWatts as a function of the incident velocity and the electric load selected. In dashed lines Eq. 4.60 is plotted which yields the maximum efficiency $\eta_{E}$ for each value of $U^{*}$. Highlighted as a black dot is given the maximum global efficiency $\eta_{E}^{G, \text { max }}$ which is obtained at a given value of $\beta_{\max }^{G}$ and $U_{\text {max }}^{* G}$ as given by Eqs. 4.62 and 4.63 respectively.

the site (for an electric load of $R_{L}=1 /\left(C_{p} \omega_{N}\right)$ ). This will yield the maximum power efficiency at such velocity. Taking this into account, the mass of the prism, its size and the oscillating frequency should be chosen accordingly. Finally, regarding the losses as parasitic damping, the ratio between the mechanical damping $\zeta$ and the electrical damping $k_{E}$ should be small to minimize the mechanical dissipation. When dealing with piezoelectric energy harvesters that take advantage of transverse galloping phenomenons, one of the main concerns is that for typical applications the value of $k_{E}$ is small and thus, the efficiency may be affected importantly if parasitic damping is not minimized.

Also, to assure a broadband character and optimize the power harvested at any value of airspeed, the electric load should be adapted continuously. Modifying the electric load resistance, the power harvested will always be maximum for each condition of the incident flow velocity. 


\subsection{Enhancing mechanical energy extraction using a dual mass system}

The use of a dual mass system to enhance the energy extraction from vibration sources was first proposed by Tang and Zuo (2011). They considered the case of a linear vibrating system where one of the masses was excited by an external oscillating force. Using the concept of a dual mass system, Nishi (2013) proposed to enhance the power extraction from vortex-induced vibrations carrying out a theoretical study to examine the energy extraction through VIV from a dual mass system. VIV fluid forces on the main mass were described by a wake oscillator model finding enhancement of power extraction by the dual mass system for appropriate combination of the dual mass parameters.

Here the possibility of enhancing the energy extraction is discussed as well as its efficiency using transverse galloping by means of a dual mass system. As it will be shown in detail later, in the dual mass system a secondary mass (not exposed to the flow) is elastically connected to the galloping body whose oscillation causes the secondary mass to oscillate. By correctly matching the secondary mass and the stiffness of the elastic connection between both masses, additional energy can be harvested from the flow; thereby increasing the overall system efficiency. Three possible dual-mass configurations will be studied hereafter. They are characterized by the location of the generator used to extract energy. In the first configuration (C1), the generator was placed between the secondary mass and a fixed wall; in the second configuration $(\mathrm{C} 2)$, the generator was located in between the main and the secondary masses. The third configuration (C3) situated the generator in between a fixed wall and the primary mass (see Fig. 4.14).

The main goal of this study is to investigate the mechanical configuration of the dual-mass system that maximizes the energy harvested of the incoming flow. To this end, a simplified two-degree-offreedom mathematical model is introduced, where fluid forces are described taking into account the quasi-steady hypothesis, as suggested by Parkinson (1989). The mathematical model is approximately solved by applying the standard Harmonic Balance Method, and analyzed in detail. As a novelty, the analysis is mainly focused on the impact of the dual-mass parameters (those related to the mass of the secondary mass and the stiffness of the elastic connection between the galloping body and the secondary mass) on the efficiency of energy harvested. Results indicate that there are dual-mass configurations which improve the energy harvested with respect to that of the single mass system as well as the existence of configurations in which the efficiency of the energy harvested maximized across in a broader amount of values of the incident flow velocity. In this sense, the system becomes more broadband which is an interesting result when involving real flows which may have varying incident velocities.

Results in this Section 4.5 have been published in Enhanced mechanical energy extraction from transverse galloping using a dual mass system, Journal of Sound and Vibration Volume 339, 17 March 2015, Pages 290-303.

\subsubsection{Mathematical model study for TG using a dual mass system for configuration $\mathrm{C} 1$}

A simplified dynamical system is considered, consisting of a spring-mounted prismatic body (galloping body) of mass $m_{1}$ immersed in an incoming flow and a secondary elastically supported mass $m_{2}$ (dualmass) which is not exposed to the aforementioned flow. They are linked by two linear springs, $k_{1}$ which connects the galloping body to a fix wall and $k_{2}$, which is situated between the galloping body and the dual-mass (see Fig. 4.14a, C1 configuration). The system is completed with a viscous damper, $c_{1}$, which is set between the fixed wall and the galloping body and an electrical generator which is represented, for simplicity, as another viscous damper $c_{E}$ located between the wall and the dual-mass. 


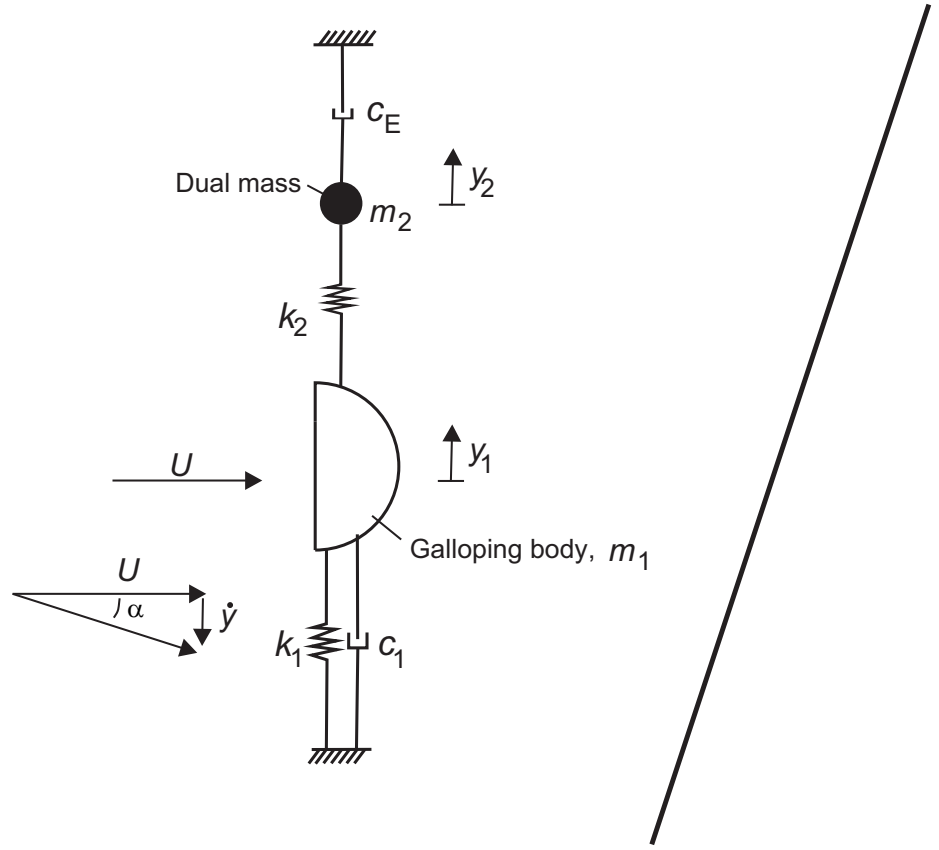

CONFIGURATION C1

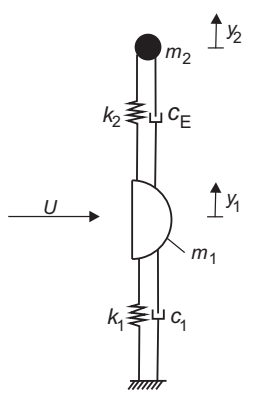

CONFIGURATION C2

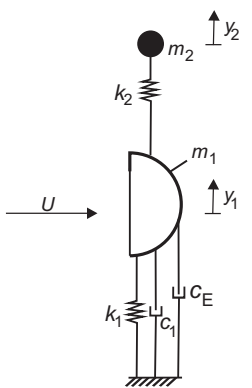

CONFIGURATION C3

Figure 4.14: Defining sketch of the three possible dual-mass configurations which are differentiated by the situation of the harvesting damper.

This configuration can be understood as a two degree of freedom system where the prismatic body (galloping body) is subject to the influence of the incident flow and is prone to transverse galloping whereas the secondary mass (dual-mass) does not suffer such influence and its vibrations are caused by its connection to the galloping body. All previous mechanical properties are defined per unit length of the galloping body.

\subsubsection{Mathematical model}

Balance between inertia, damping, stiffness, and fluid forces in the system yield the following system of two ordinary differential equations:

$$
\begin{gathered}
m_{1} \ddot{y}_{1}+c_{1} \dot{y}_{1}+k_{1} y_{1}+k_{2}\left(y_{1}-y_{2}\right)=\frac{1}{2} \rho U^{2} D C_{y}, \\
m_{2} \ddot{y}_{2}+k_{2}\left(y_{2}-y_{1}\right)+c_{E} \dot{y}_{2}=0,
\end{gathered}
$$

where $y_{1}$ denotes the position of galloping body, $y_{2}$ the position of dual-mass, with respect to the zero equilibrium position of the system, $\rho$ is the fluid density, $U$ is the undisturbed velocity of the incident flow, $D$ is the characteristic dimension of the cylinder prone to gallop normal to the flow, $C_{y}$ is the instantaneous fluid force coefficient on the galloping body in the transverse direction to the incident flow and finally, the dot symbol stands for differentiation with respect to physical time $t$.

Introducing the following dimensionless variables, $Y_{1}=y_{1} / D, Y_{2}=y_{2} / D, \omega_{1}=\left(k_{1} / m_{1}\right)^{\frac{1}{2}}$, frequency ratio $\Omega=\left(k_{2} / k_{1}\right)^{\frac{1}{2}}$, dimensionless parasitic damping coefficient $\zeta_{1}=c_{1} /\left(2 m_{1} \omega_{1}\right)$ dimensionless damping coefficient of the generator $\zeta_{E}=c_{E} /\left(2 m_{1} \omega_{1}\right)$, mass ratio of the primary mass $m^{*}=m_{1} /\left(\rho D^{2}\right)$, ratio between masses $\mu=m_{2} / m_{1}$, dimensionless time $\tau=\omega_{1} t$, and reduced velocity $U^{*}=U /\left(\omega_{1} D\right)$ it is possible to write the dimensionless equations for the system dynamics:

$$
Y_{1}^{\prime \prime}+2 \zeta_{1} Y_{1}^{\prime}+Y_{1}+\Omega^{2}\left(Y_{1}-Y_{2}\right)=\frac{U^{* 2}}{2 m^{*}}\left(a_{1} \frac{Y_{1}^{\prime}}{U^{*}}+a_{3}\left(\frac{Y_{1}^{\prime}}{U^{*}}\right)^{3}\right)
$$




$$
\mu Y_{2}^{\prime \prime}+\Omega^{2}\left(Y_{2}-Y_{1}\right)+2 \zeta_{E} Y_{2}^{\prime}=0,
$$

where in this case the prime represents differentiation with respect to the dimensionless time. Note that the only external force exerted on the system is the fluid dynamic force.

\subsubsection{Galloping response}

Eqs. 4.69a and 4.69b contain six parameters $\left(\zeta_{1}, \zeta_{E}, \mu, \Omega, m^{*}\right.$ and $\left.U^{*}\right)$, meaning that the solution manifold exists inside a 6 -dimensional space. To gather a better understanding of the system behavior in this 6-dimensional space, the use of theoretical analysis able to yield analytical solutions (valid within the set of prescribed hypothesis) is deemed to be beneficial because it facilitates the visualization of the global trends. If it is assumed that the nonlinear term of the fluid force is small $\left(m^{*} U^{*} \gg 1\right)$ and that values of $\zeta_{E}$ are moderate, the Harmonic Balance Method of first order (HBM) can be employed assuming that the steady response is harmonical, solutions have the following form

$$
Y_{1}=A^{*} \sin \left(\omega^{*} \tau\right), \quad Y_{2}=B^{*} \sin \left(\omega^{*} \tau+\phi\right),
$$

where the four unknowns, $\omega^{*}=\omega / \omega_{1}, A^{*}, B^{*}$ and $\phi$ have to be solved.

From Eq. $4.69 \mathrm{~b}$, it is possible to obtain the relation between dual-mass and galloping body amplitudes, as a linear two degrees of freedom response:

$$
K^{*}=\frac{B^{*}}{A^{*}}=\frac{\Omega^{2}}{\sqrt{\left(-\mu \omega^{* 2}+\Omega^{2}\right)^{2}+4 \zeta_{E}^{2} \omega^{* 2}}} .
$$

The phase delay $\phi$ of the harmonic response between the dual-mass and the galloping body is given by

$$
\begin{aligned}
& \cos \phi=\frac{-\mu \omega^{* 2}+\Omega^{2}}{\sqrt{\left(-\mu \omega^{* 2}+\Omega^{2}\right)^{2}+4 \zeta_{E}^{2} \omega^{* 2}}}, \\
& \sin \phi=\frac{-2 \zeta_{E} \omega^{*}}{\sqrt{\left(-\mu \omega^{* 2}+\Omega^{2}\right)^{2}+4 \zeta_{E}^{2} \omega^{* 2}}} .
\end{aligned}
$$

All parameters involved for the determination of $K^{*}$ and $\phi$ depend only on mechanical properties $\left(\Omega, \mu, \zeta_{1}\right.$ and $\left.\zeta_{E}\right)$ which can be defined a priori at the design stage; the only unknown parameter is the dimensionless oscillating frequency $\omega^{*}$ which is obtained after applying HBM to Eq. 4.69a and equating sine terms

$$
D\left(\omega^{*}\right)=-\omega^{* 2}+1+\Omega^{2}\left(1-K^{*} \cos \phi\right)=0 .
$$

Eq. 4.73 can be solved numerically along with Eqs. 4.71 and 4.72 , yielding $\omega^{*}$, which may have two solutions as it corresponds to a linear 2 degree of freedom system obtaining for each case different values of $K^{*}$ and $\phi$. In a steady state solution, the system may only vibrate at one of the previous frequencies depending on the initial conditions of the system and in concordance, different galloping response as well as different values of energy harvesting efficiency may be obtained (thus yielding two modes of vibration). Equating cosine terms in Eq. 4.69a and truncating cubic cosine terms $\left[\cos \left(\omega^{*} \tau\right)^{3} \approx 3 / 4 \cos \left(\omega^{*} \tau\right)\right]$ the amplitude response $A^{*}$ is

$$
A^{*}=\left(\frac{4 U^{*}}{3 a_{3} \omega^{* 2}}\left(4 m^{*} \zeta_{T}-a_{1} U^{*}\right)\right)^{1 / 2},
$$

where $\zeta_{T}$ stands for

$$
\zeta_{T}=\zeta_{1}+\zeta_{E} K^{* 2},
$$


which is very useful in the ongoing discussion as it represents the equivalent damping of the whole Dual Mass system. $A^{*}$ depends on the reduced flow velocity $U^{*}$, the galloping body's geometry $\left(a_{1}\right.$ and $\left.a_{3}\right)$, the mechanical properties $\left(m^{*}, \mu, \Omega, \zeta_{1}\right)$, on the generator $\left(\zeta_{E}\right)$ and whether the system is vibrating at one or the other mode. In addition, note that $A^{*}$ has physical sense (i.e $A^{*}>0$ ) when the reduced velocity exceeds a critical value $U_{g}^{* D M}$ which is the reduced velocity at which the system starts galloping meaning that the destabilizing effect of the fluid force equals the stabilizing effect of mechanical damping

$$
U_{g}^{* D M}=\frac{4 m^{*} \zeta_{T}}{a_{1}}
$$

For fixed mechanical (or design) parameters, $\omega^{*}, K^{*}$ and $\phi$ are obtained numerically from Eqs. 4.71, 4.72 and 4.73. Eq. 4.74 yields $A^{*}$, thus solving analytically the model of Dual Mass energy harvester. It is is important to keep in mind that this solution is valid only whenever the quasi-steady hypothesis holds, when the effective reduced velocity $V^{*}=U^{*} / \omega^{*}$ is high enough. It has been checked that HBM approximation is quite robust, and comparisons with numerical solutions of Eqs. 4.69 have been quite close even for values of $m^{*} U^{*}$ of order unity.

\subsubsection{Energy harvesting efficiency}

Once the solution of Eq. 4.69 has been derived, a conversion factor (or efficiency) is defined as the time-averaged power $P_{E}$ dissipated at $c_{E}$ divided by the flux of energy across the section of the prism:

$$
\eta^{D M}=\frac{P_{E}}{\frac{1}{2} \rho U^{3} D},
$$

where $P_{E}$ is

$$
P_{E}=\frac{1}{T} \int_{0}^{T} c_{E} \dot{y}_{2}^{2} d t=2 m_{1} \omega_{1} \zeta_{E}\left(\omega_{1} D\right)^{2}\left\langle\dot{Y}_{2}^{2}\right\rangle,
$$

and $T$ and $<$. $>$ mean respectively the time period of oscillation of the dual-mass system and averaged value. It is possible to rewrite the efficiency $\eta^{D M}$ as:

$$
\eta^{D M}=\frac{4 m^{*} \zeta_{E}\left\langle\dot{Y}_{2}^{2}\right\rangle}{U^{* 3}}
$$

and from Eqs. 4.78 and 4.79 one gets $\eta^{D M}$ by substituting the averaged value of $\left\langle\dot{Y}_{2}^{2}\right\rangle$

$$
\eta^{D M}=\frac{2 m^{*} \zeta_{E} B^{* 2} \omega^{* 2}}{U^{* 3}}
$$

Substituting $B^{*}$ (from Eqs. 4.71 and 4.74) into Eq. 4.80 an analytical expression for the efficiency as a function of the mechanical properties $\left(\mu, \Omega, \zeta_{1}, \zeta_{E}\right)$, the geometry of the galloping body $\left(a_{1}, a_{3}\right)$ and the reduced velocity $U^{*}$ is obtained,

$$
\eta^{D M}=\frac{8 m^{*} \zeta_{E}\left(4 m^{*} \zeta_{T}-a_{1} U^{*}\right) \Omega^{4}}{a_{3} U^{*^{2}}\left(\left(-\mu \omega^{* 2}+\Omega^{2}\right)^{2}+4 \zeta_{E}^{2} \omega^{* 2}\right)} .
$$

Differentiating Eq. 4.80 or Eq. 4.81 with respect to $U^{*}$ and equating to zero, the maximum efficiency is obtained at twice the critical velocity when the system starts galloping $\left(U_{\eta_{\max }^{* D M}}^{*}=2 U_{g}^{* D M}\right)$.

$$
U_{\eta_{\max }^{* D}}^{* D}=\frac{8 m^{*} \zeta_{T}}{a_{1}}
$$

Introducing $U_{\eta_{\max }^{* D M}}^{* D}$ into Eq. 4.81, it yields that the maximum efficiency that can be obtained is 


$$
\eta_{\max }^{D M}=-\frac{a_{1}^{2}}{6 a_{3}} \frac{\zeta_{E}}{\zeta_{T}} K^{* 2}
$$

A universal curve for the efficiency can be introduced using two new normalized variables, $\tilde{\eta}=$ $\eta^{D M} / \eta_{\max }^{D M}$ and $\tilde{U}=U^{*} / U_{g}^{* D M}$ and substituting them into Eq. 4.81

$$
\tilde{\eta}=-\frac{4(1-\tilde{U})}{\tilde{U}^{2}}
$$

With these renormalized variables, it is possible to collapse the efficiency curves for all mechanical configurations into a single curve (Eq. 4.84) providing information about the transverse galloping response over a range of reduced incident velocities. From Fig. 4.15, once galloping is started $(\tilde{U}=1)$, the normalized efficiency increases rapidly with the normalized reduced velocity until a value of $\tilde{U}=2$ is reached obtaining a value of the normalized efficiency of $\tilde{\eta}=1$, then the normalized efficiency diminishes slowly with $\tilde{U}$.

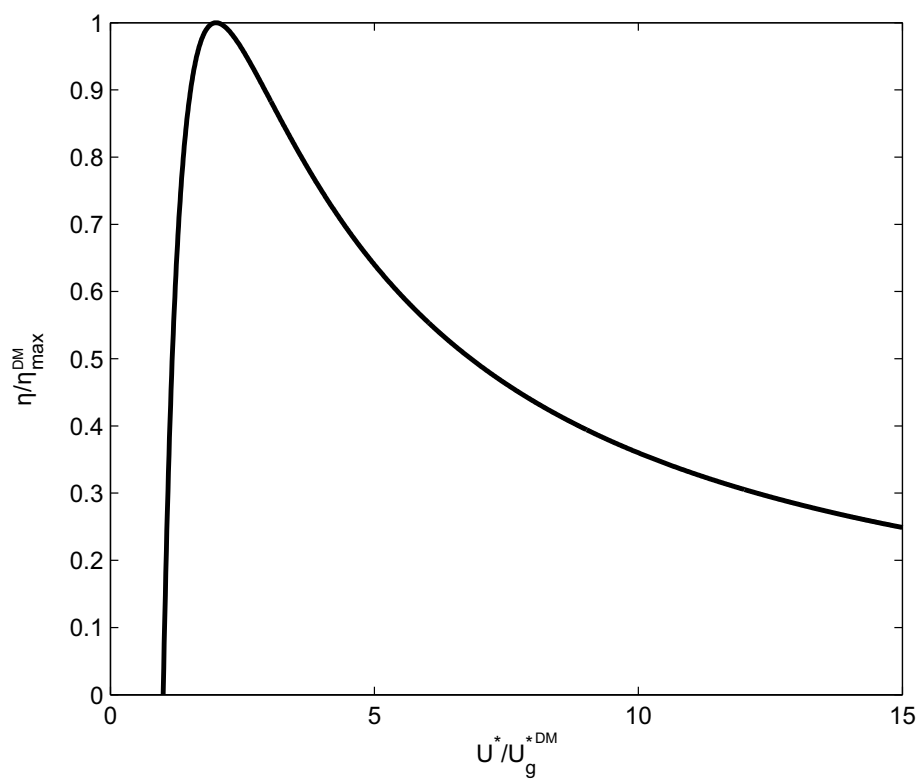

Figure 4.15: Universal efficiency curve valid for all mechanical configurations. $\tilde{\eta}=\eta^{D M} / \eta_{\max }^{D M}$ and $\tilde{U}=U^{*} / U_{g}^{* D M}$.

When the dual mass effect is minimized, for example making $\Omega=\infty, \mu=0$, the solution for the single mass configuration presented in Barrero-Gil et al., 2010 is recovered from Eqs. 4.71 to 4.74 and Eq. 4.83

$$
\begin{gathered}
K^{*}=\frac{B^{*}}{A^{*}}=1, \\
\phi=0, \\
\omega_{S M}^{*}=1, \\
A_{S M}^{*}=\left(\frac{4 U^{*}}{3 a_{3}}\left(4 m^{*}\left(\zeta_{1}+\zeta_{E}\right)-a_{1} U^{*}\right)\right)^{1 / 2}, \\
\eta_{\max }^{S M}=-\frac{a_{1}^{2}}{6 a_{3}} \frac{\zeta_{E}}{\zeta_{1}+\zeta_{E}},
\end{gathered}
$$

In the ongoing discussion, the dual-mass system is compared to the single mass counterpart as defined by Eq. 19. in order to find mechanical configurations of $\mu$ and $\Omega$ that yield better energy 
harvesting efficiency.

\subsubsection{Dual Mass effect}

From the practical side, so as to harness the maximum amount of energy, one would be interested in having (i) $\eta_{\max }^{D M}$ as high as possible (maximum efficiency) and having (ii) $U_{g}^{* D M}$ large in order to have a broadband character of efficiency dependence with the reduced velocity. Thus, two differentiated strategies exist for the design of the Dual Mass system.

\subsection{Looking for high $\eta_{\max }^{D M}$}

If there is no parasitic damping $\left(\zeta_{1}=0\right)$, from Eqs. 4.83 and 4.85 e one may see that the maximum available efficiency for the dual-mass and single mass systems is,

$$
\eta_{\max }^{D M}=\eta_{\max }^{S M}=-\frac{a_{1}^{2}}{6 a_{3}}=\eta_{F}
$$

where $\eta_{F}$ stands for the hydrodynamic efficiency which only depends on the geometry of the galloping body and therefore, when there is no parasitic damping, the maximum efficiency is $\eta_{F}$ for all possible mechanical configuration. In other words, it is not possible to extract power ideally $\left(\zeta_{1}=0\right)$, from the incoming flow, with better efficiency than for the single mass system using a dual-mass system. However, $\eta_{\max }^{D M}$ and $\eta_{\max }^{S M}$ cannot be (in general) as large as $\eta_{F}$ since the maximum power harnessable is less due to the dissipation of power as parasitic damping. This parasitic damping $\left(\zeta_{1}\right)$ can have relative importance (compared to $\zeta_{E}$ ) when building real transverse galloping energy harvesters. From Eqs. 4.83 and $4.85 \mathrm{e}$ a harnessable factor that relates the hydrodynamic efficiency with the maximum harnessable efficiency for both single mass and dual-mass systems can be defined.

$$
\begin{gathered}
\gamma_{D M}=\frac{\eta_{\max }^{D M}}{\eta_{F}}=\frac{\zeta_{E} K^{* 2}}{\zeta_{T}}=\frac{\frac{\zeta_{E}}{\zeta_{1}} K^{* 2}}{1+\frac{\zeta_{E}}{\zeta_{1}} K^{* 2}}, \\
\gamma_{S M}=\frac{\eta_{\max }^{S M}}{\eta_{F}}=\frac{\frac{\zeta_{E}}{\zeta_{1}}}{1+\frac{\zeta_{E}}{\zeta_{1}}} .
\end{gathered}
$$

The goal of the ongoing discussion is to show that it is possible to enhance the dual-mass maximum efficiency compared to the single mass one when parasitic damping exists $\left(\zeta_{1} \neq 0\right)$. Thus, it is convenient to introduce a dual mass factor that relates the maximum efficiency of both Dual and Single Mass systems

$$
\psi_{D M}=\frac{\eta_{\max }^{D M}}{\eta_{\max }^{S M}}=\frac{\gamma_{D M}}{\gamma_{S M}}=\frac{\left(1+\frac{\zeta_{E}}{\zeta_{1}}\right) K^{* 2}}{1+\frac{\zeta_{E}}{\zeta_{1}} K^{* 2}} .
$$

Observe that $\psi_{D M}$ is not dependent on the geometry of the galloping body since this dependence is fully collected in $\eta_{F}$. If $\psi_{D M}$ were larger than one then the corresponding dual-mass system would present a better maximum efficiency. From Eq. 4.89, the maximum value of $\psi_{D M}^{\max }$ that can be obtained is

$$
\psi_{D M}^{\max }=\gamma_{S M}^{-1}=1+\frac{\zeta_{1}}{\zeta_{E}},
$$

which is the asymptotic value of Eq. 4.89 when $K^{*} \gg 1$. As it can be seen in Eq. 4.89, the improvement due to the dual-mass effect (that is $\psi_{D M}>1$ ) comes from the fact that $K^{*}$ can be augmented beyond 1. As both masses move at the same frequency, the power dissipated at each damper is proportional to $\zeta_{E} B^{* 2}$ and to $\zeta_{1} A^{* 2}$ respectively, and the system is capable of dissipating the same global power as in the single mass configuration, but proportionally the power dissipated 
in $\zeta_{E}$ has been enhanced as long as $K^{*}>1$. From Eq. 4.90, the potential improvement is larger as $\zeta_{1} / \zeta_{E}$ is increased, therefore the dual-mass configuration is indicated for situations in which $\zeta_{1} / \zeta_{E}$ is at least of the same order.

\subsection{Looking for a broadband-type response}

Real flows usually involve variations in the incident flow velocity. From a practical viewpoint it is interesting to have a broadband-type response with respect to variations of the incoming flow speed. As seen from the universal efficiency curve (see Fig. 4.15), high efficiency values are given around $\tilde{U}=2$. If it is desirable to have a normalized efficiency superior to a given one, for example, $\tilde{\eta}>x$ then the normalized range of reduced velocities where this happens is $\Delta \tilde{U}=\Delta U^{*} / U_{g}^{* D M}=4 x^{-1}(1-x)^{1 / 2}$ as obtained from Eq. 4.84. To analyze the broadband behavior of the dual-mass configuration, a new variable relating the critical galloping velocity of dual-mass and single mass systems is introduced

$$
\chi_{D M}=\frac{U_{g}^{* D M}}{U_{g}^{* S M}}=\frac{1+\frac{\zeta_{E}}{\zeta_{1}} K^{* 2}}{1+\frac{\zeta_{E}}{\zeta_{1}}},
$$

which is only dependent on the mechanical parameters of the dual-mass system. Then the reduced galloping velocities that fulfill the prescribed condition for the efficiency is

$$
\Delta U^{*}=4 x^{-1}(1-x)^{1 / 2} U_{g}^{* D M}=4 x^{-1}(1-x)^{1 / 2} U_{g}^{* S M} \chi_{D M}
$$

This last equation shows that when $\chi_{D M}>1$, the range of reduced velocities fulfilling the requested condition for the efficiency has been enlarged proportional to $\chi_{D M}$ with respect to the single mass configuration and thus the dual-mass response is more broadband.

\subsubsection{5 $\psi_{D M}$ and $\chi_{D M}$ dependence on the mechanical parameters of the dual-mass sys- tem}

$\psi_{D M}$ and $\chi_{D M}$ give information about the properties of the dual-mass configuration in comparison to the original Single Mass system. They depend directly on the ratio of amplitudes between the dual-mass and the galloping body $\left(K^{*}\right)$, on the parasitic damping $\left(\zeta_{1}\right)$ and on the generator $\left(\zeta_{E}\right)$. $K^{*}$, on the other hand, depends on $\mu, \Omega$ and $\zeta_{E}$ as well as the frequency $\left(\omega^{*}\right)$ at which the system is vibrating given by solving Eq. 4.73 from the two possible solutions (modes). Interesting configurations for $\mu$ and $\Omega$ are those that provide values of $\psi_{D M}$ and $\chi_{D M}$ larger than one, since it is desirable to enhance the maximum efficiency $\left(\psi_{D M}>1\right)$ as well as to provide better efficiency for more values of the reduced velocity $\left(\chi_{D M}>1\right)$. Fig. 4.16 shows contour plots for $\psi_{D M}$ as a function of $\Omega$ and $\mu$ for given values of $\zeta_{E}=0.05$ and $\zeta_{1}=0.0125$ and for each of the two possible modes. From Fig. 4.16 a region can be observed in the parameter space $(\mu-\Omega)$ where $\psi_{D M}$ is higher than one, meaning that the dual mass system tuned in that way increases the maximum efficiency achievable; note that values of $\psi_{D M}$ under 1 means that the dual-mass systems introduce additional damping and behave like a traditional Tuned Mass Damper (Abdelkefi et al., 2013c). Values of $\zeta_{E}$ and $\zeta_{1}$ don't change the qualitative character of the results, only the maximum achievable values.

With regard to $\chi_{D M}$ behaviour, from Fig. 4.17 it can be seen that there is also a region where $\chi_{D M}$ is larger than one and thus the system is more broadband. On the other hand, $\chi_{D M}<1$ means it is less broadband and therefore, for small changes in the reduced velocity from the optimal obtained at $U^{*}=2 U_{g}^{* D M}$ leads rapidly to worse values of the efficiency.

$\psi_{D M}$ and $\chi_{D M}$ behavior can be explained with the aid of Eqs. 4.89 and 4.91 since a causal relation between $\psi_{D M}$ and $\chi_{D M}$ with $K^{*}$ is derived

$$
\psi_{D M} \chi_{D M}=K^{* 2} .
$$


(a)

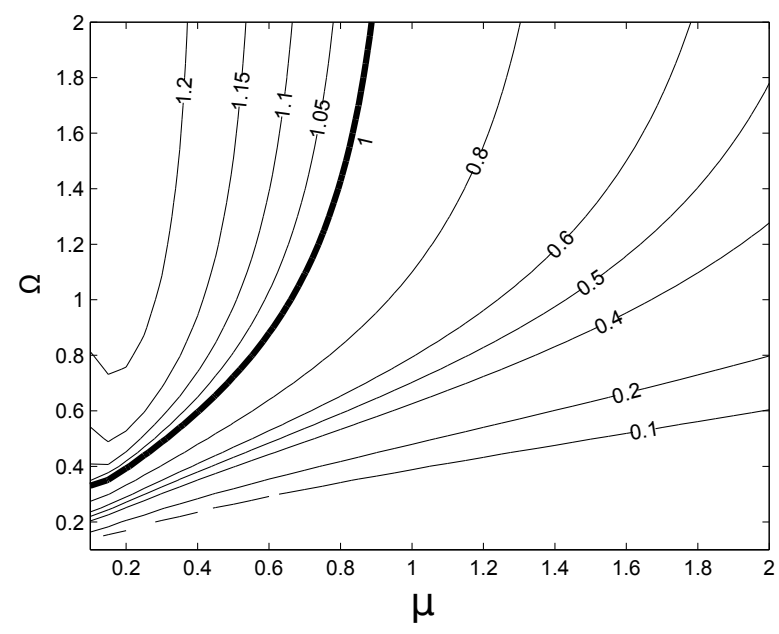

(b)

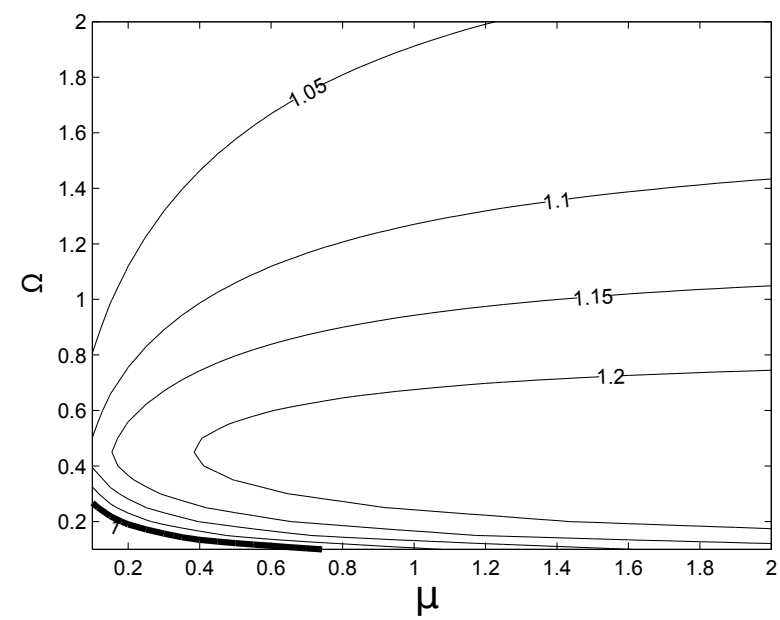

Figure 4.16: $\psi_{D M}$ dependence with mechanical properties of the dual-mass systems $(\Omega, \mu)$ for the first (a) and second (b) mode. The first mode always involves values of $\omega^{*}>1$ whereas the second one involves values of $\omega^{*}<1$.

(a)

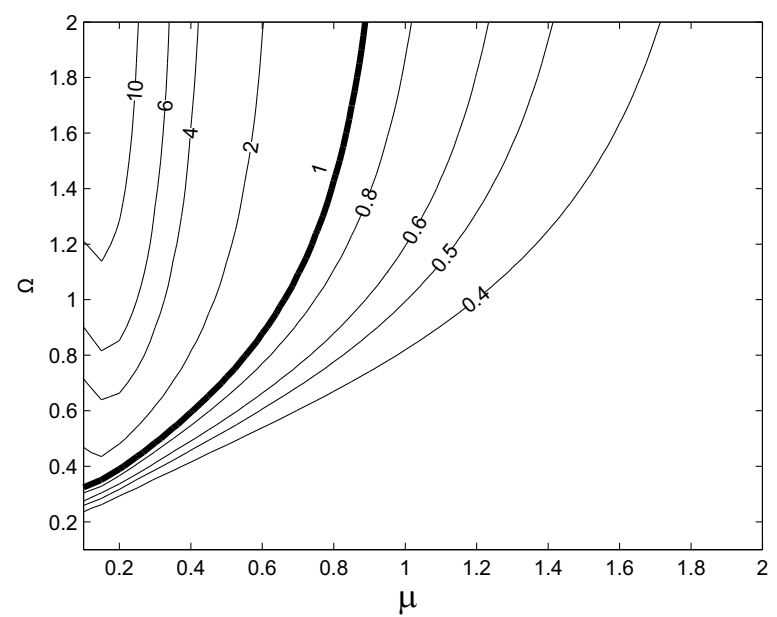

(b)

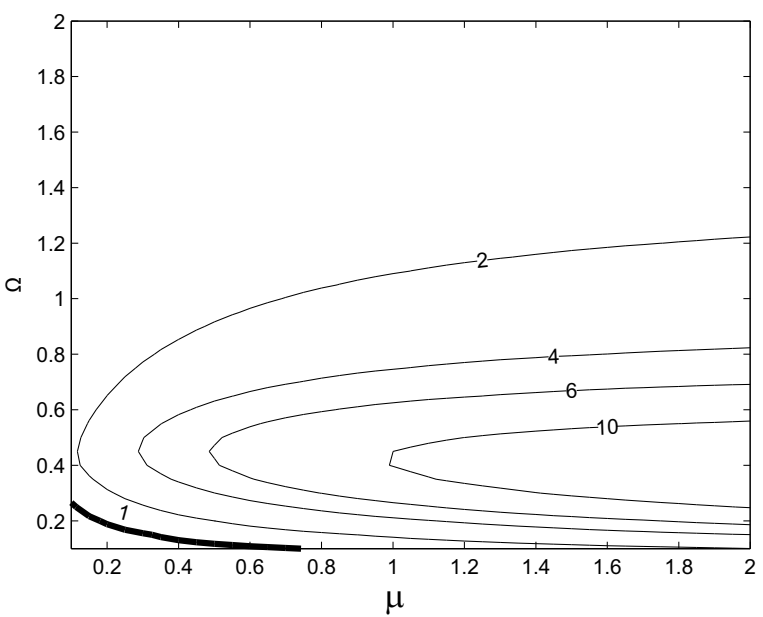

Figure 4.17: $\chi_{D M}$ dependence with mechanical properties of the dual-mass systems $(\Omega, \mu)$ for the first mode $\omega^{*}>1$ (a) and for the second mode $\omega^{*}<1$ (b).

If $K^{*}>1$, then $\psi_{D M}>1$ and $\chi_{D M}>1$, whereas if $K^{*}<1, \psi_{D M}$ and $\chi_{D M}$ will also be smaller than one. Therefore, there are only two possible regions concerning $K^{*}$ as the trend of $\psi_{D M}$ and $\chi_{D M}$ only depend on this amplitude ratio.

The possibility of implementing more than two oscillating masses in the system has been analyzed using the same methodology (details are not described here for the sake of brevity). The conclusion, regarding its spectral behavior, is that, if a realistic set of design parameters is considered, the broadband character of the system response cannot be improved significantly when more than two masses are used. In any case, these marginal gains would be offset by the increase in the system mechanical complexity. Regarding the peak efficiency, it is the same as the dual-mass system and the same as the single-mass system when no parasitic damping exists. 


\begin{tabular}{|c|c|}
\hline SINGLE MASS & DUAL MASS \\
\hline \hline$m^{*}=10$ & $m^{*}=10$ \\
$\zeta_{1}=0.0125$ & $\zeta_{1}=0.0125$ \\
$\zeta_{E}=0.05$ & $\zeta_{E}=0.05$ \\
- & $\mu=0.45$ \\
- & $\Omega=0.9$ \\
$a_{1}=0.79$ & $a_{1}=0.79$ \\
$a_{3}=-0.19$ & $a_{3}=-0.19$ \\
$D=0.05 m$ & $D=0.05 m$ \\
$L=1 \mathrm{~m}$ & $L=1 \mathrm{~m}$ \\
$\rho=1000 \mathrm{~kg} / \mathrm{m}^{3}$ & $\rho=1000 \mathrm{~kg} / \mathrm{m}^{3}$ \\
$\omega_{1}=2.5 \mathrm{rad} / \mathrm{s}$ & $\omega_{1}=2.5 \mathrm{rad} / \mathrm{s}$ \\
\hline
\end{tabular}

Table 4.4: Physical parameters for the single mass and dual-mass systems considered in the presented practical example.

\subsubsection{Practical example}

For illustrative purposes, a practical example is shown here. As it will be seen, thanks to the dual-mass arrangement, the maximum efficiency and the broadband character response with respect to a baseline single mass configuration is enhanced. Also, a comparison between the HBM method proposed and numerical solutions has been made. For this particular example, the single mass energy harvester used as a model has the following parameters, $m^{*}=10, \zeta_{1}=0.0125$ and $\zeta_{E}=0.05$ (for the whole definition of the system, see Table 4.4). This system could resemble a TG energy harvester immersed in water, as the mean density of the galloping body is slightly superior to the density of the water. The design criteria for the dual-mass $\mathrm{C} 1$ configuration has been that $\chi_{D M} \approx 2$ and therefore, $U_{g}^{* D M} \approx 2 U_{g}^{* S M}$. With the previous condition, values of $\mu=0.45$ and $\Omega=0.9$ are situated near the isoline for the value $\chi_{D M}$ selected (see Fig. 4.17). With the chosen values of the mechanical parameters a value of $\psi_{D M}=1.122$ is reached (see Fig. 4.16).

(a)

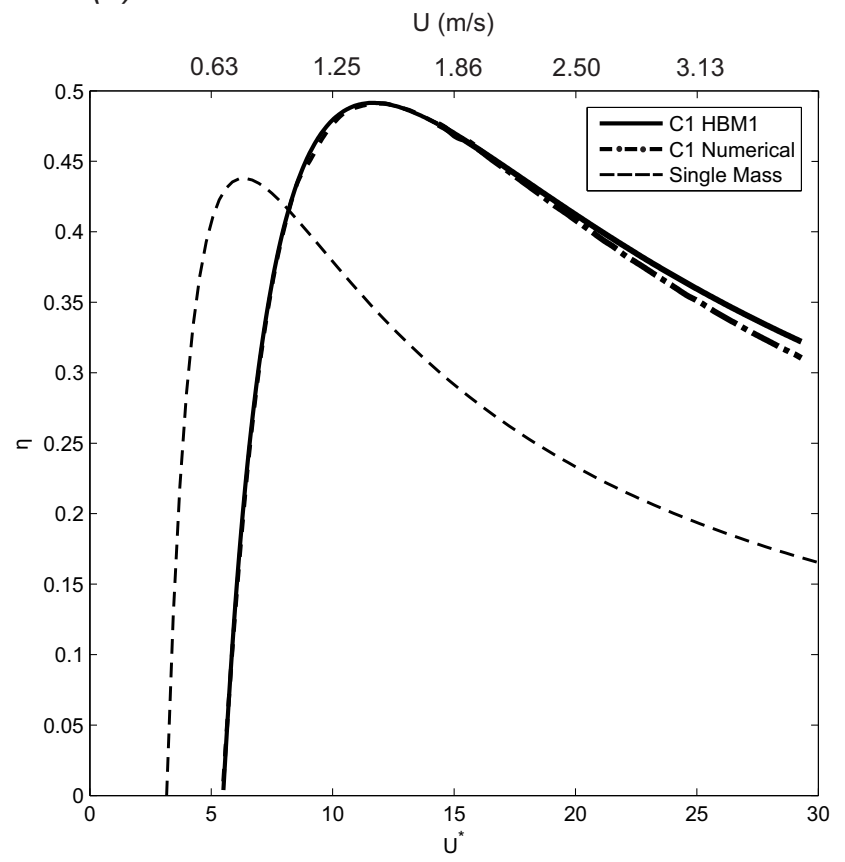

(b)

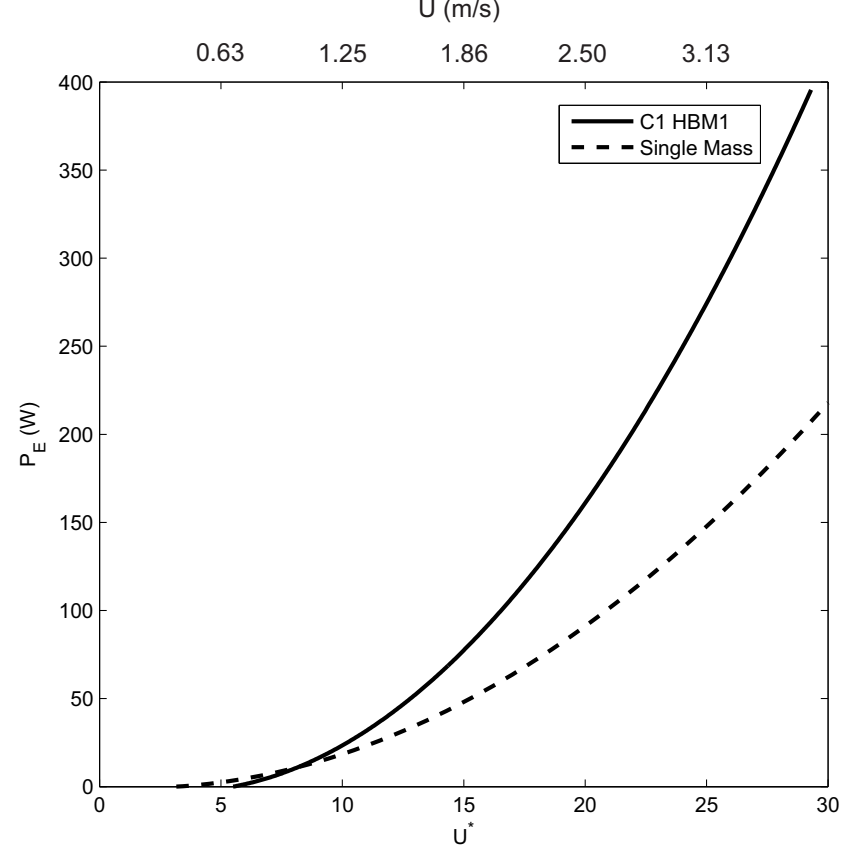

Figure 4.18: Left: Efficiency variation with the reduced velocity for the single mass system with the dual-mass system and dual mass-system (HBM and numerical solution). Right: power extracted for the single mass and dual-mass configurations. 
In Fig. 4.18a, the efficiency variation with the reduced velocity (flow speed) is plotted for the previous C1 configuration; this variation has been obtained with the HBM method as well as by numerical integration of Eq. 4.69. Both solutions are close to each other and only a slight divergence between solutions can be observed for large values of $U^{*}$ which shows that the HBM method gives good approximations even when $m^{*} \sim 1$. Comparing the $\mathrm{C} 1$ dual-mass system to the original Single Mass one, the maximum efficiency has been improved by $12.2 \%$. In addition, the efficiency dependance with the reduced velocity has been reduced in the sense that the system is more efficient in a larger range of reduced velocities; for example, in the single mass configuration $\eta$ is higher than 0.4 in the range $5<U^{*}<9$ approximately, whereas for the dual-mass system the range of reduced velocities where $\eta>0.4$ is $8<U^{*}<21$ approximately. In Fig. $4.18 \mathrm{~b}$ the dimensional power harvested is plotted as a function of the reduced velocity (flow speed). As it can observed, the power extracted is always superior (except for the initial velocities) for the dual-mass configuration. This example shows that it is possible to enhance the power extraction by changing the dual-mass parameters.

For illustration purposes and to gain better physical insight, a potential practical implementation of a dual-mass system is shown in Fig. 4.19. Note that the system displayed in the figure corresponds to the first configuration (C1). In operation the system would work as follows: under the action of the fluid flow, the galloping body oscillates perpendicular to the direction of the flow and drives the secondary mass (or dual mass). This secondary mass then drives the permanent magnet of a linear electromagnetic generator (note that for simplicity, the electromagnetic generator has been considered as a linear damper in the mathematical model). Relative motion between the magnet and the coil of the generator produces electrical current. Note that a dual-mass system can be implemented in a previously built galloping energy harvester with small changes (which only includes the introduction of a secondary mass and reorganizing the elements that had already been installed in the original single mass system). Then, it is possible to improve the maximum efficiency that can be harvested as well as to broaden the range of reduced velocities where such efficiency is kept high. Thus, the dual-mass concept can theoretically improve the overall performance of the galloping energy harvester with minor modifications to the installation.

(a)

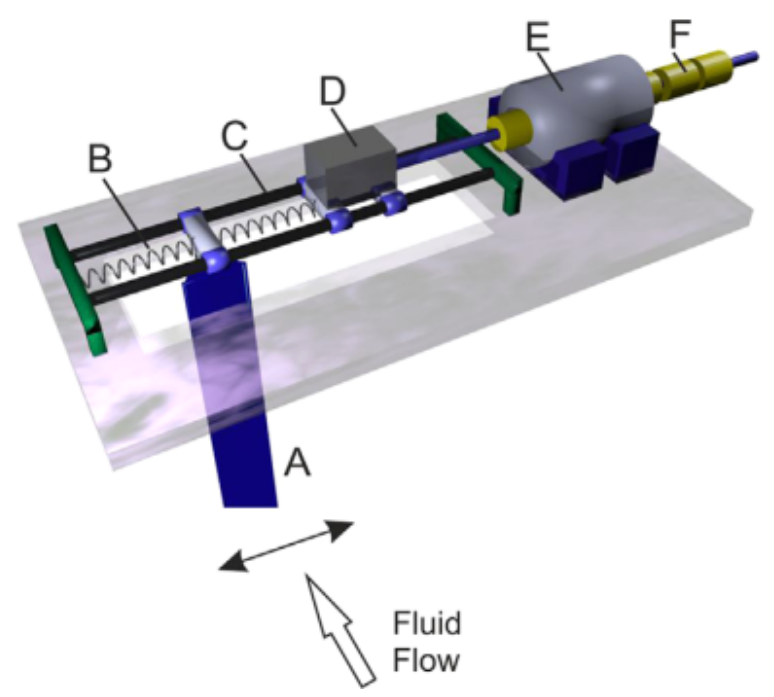

(b)
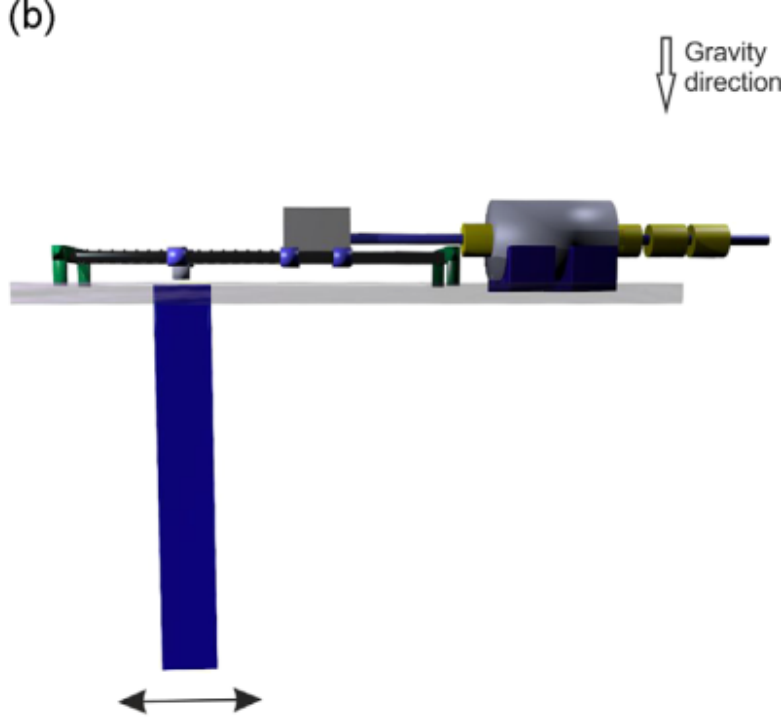

Figure 4.19: Sketch showing a potential practical realization, which includes a galloping prism (A), springs (B), a linear guide (C) transverse to the flow direction, the secondary mass (D), the stator (coil) part of the electromagnetic generator, and a permanent magnet array $(\mathrm{F})$. A 3D view is shown in (a) whereas a front view is shown in (b). 


\subsubsection{Mathematical model study for TG using a dual mass system for configuration C2 and C3}

\subsubsection{C2 Configuration}

In this section the $C 2$ configuration is presented as well as its most important results. For this case, the harvester damper $c_{E}$ is situated between the primary galloping body and the secondary mass (dual-mass) (Fig. 4.14b). Having these changes present, the non-dimensional equations for the $C 2$ configuration stands as such:

$$
\begin{gathered}
Y_{1}^{\prime \prime}+2 \zeta_{1} Y_{1}^{\prime}+Y_{1}+2 \zeta_{E}\left(Y_{1}^{\prime}-Y_{2}^{\prime}\right)+\Omega^{2}\left(Y_{1}-Y_{2}\right)=\frac{U^{* 2}}{2 m^{*}}\left(a_{1} \frac{Y_{1}^{\prime}}{U^{*}}+a_{3}\left(\frac{Y_{1}^{\prime}}{U^{*}}\right)^{3}\right), \\
\mu Y_{2}^{\prime \prime}+2 \zeta_{E}\left(Y_{2}^{\prime}-Y_{1}^{\prime}\right)+\Omega^{2}\left(Y_{2}-Y_{1}\right)=0
\end{gathered}
$$

Applying the HBM method supposing harmonic solutions such as those of Eq. 4.70 the following solutions are obtained for Eq. 4.94. Solving in first place Eq. 4.94b,

$$
\begin{gathered}
K^{*}=\frac{B^{*}}{A^{*}}=\frac{\sqrt{\Omega^{4}+4 \zeta_{E}^{2} \omega^{* 2}}}{\sqrt{\left(-\mu \omega^{* 2}+\Omega^{2}\right)^{2}+4 \zeta_{E}^{2} \omega^{* 2}}}, \\
\sin \phi=-\frac{2 \zeta_{E} \mu \omega^{* 3}}{\left(\Omega^{4}+4 \zeta_{E}^{2} \omega^{* 2}\right)^{1 / 2}\left(\left(\Omega^{2}-\mu \omega^{* 2}\right)^{2}+4 \zeta_{E} \omega^{* 2}\right)^{1 / 2}}, \\
\cos \phi=-\frac{\Omega^{2}\left(\Omega^{2}-\mu \omega^{* 2}\right)-4 \omega^{* 2} \zeta_{E}^{2}}{\left(\Omega^{4}+4 \zeta_{E}^{2} \omega^{* 2}\right)^{1 / 2}\left(\left(\Omega^{2}-\mu \omega^{* 2}\right)^{2}+4 \zeta_{E} \omega^{* 2}\right)^{1 / 2}},
\end{gathered}
$$

$K^{*}$ and $\phi$ are known for given $\zeta_{1}, \zeta_{E}, \mu$ and $\Omega$, except for $\omega^{*}$. Applying HBM to Eq. 4.94a and equating sine terms,

$$
D\left(\omega^{*}\right)=-\omega^{* 2}+1+\Omega^{2}\left(1-K^{*} \cos \phi\right)+2 \zeta_{E} K^{*} \sin \phi=0,
$$

Eq. 4.96 is an implicit function for $\omega^{*}$, which has two solutions corresponding to each of the possible modes. Equating cosine terms of $4.94 \mathrm{a}$ and truncating cubic terms as previously done,

$$
A^{*}=\left(\frac{4 U^{*}}{3 a_{3} \omega^{* 2}}\left(4 m^{*} \zeta_{T}-a_{1} U^{*}\right)\right)^{1 / 2} .
$$

The system starts to oscillate when the reduced velocity surpasses the critical galloping reduced velocity,

$$
U_{g}^{* D M}=\frac{4 m^{*} \zeta_{T}}{a_{1}} .
$$

expressions of Eqs. 4.97 and 4.98 are the same as for the $\mathrm{C} 1$ configuration. Only the value of $\zeta_{T}$ changes from the $\mathrm{C} 1$ to the $\mathrm{C} 2$ configuration, for this case

$$
\zeta_{T}=\zeta_{1}+\zeta_{E}\left(1+K^{* 2}-2 K^{*} \cos \phi\right) .
$$

\subsubsection{Energy harvesting efficiency}

The mean power $P_{E}$ dissipated at $c_{E}$ (now situated between the galloping body and the dual-mass) is

$$
P_{E}=\frac{1}{T} \int_{0}^{T} c_{E}\left(\dot{y}_{1}-\dot{y}_{2}\right)^{2} d t=2 m_{1} \omega_{1} \zeta_{E}\left(\omega_{1} D\right)^{2}\left\langle\left(\dot{Y}_{1}-\dot{Y}_{2}\right)^{2}\right\rangle,
$$


where $<$. $>$ means averaged value, the efficiency is then given as

$$
\eta^{D M}=\frac{2 m^{*} \zeta_{E} A^{* 2}\left(1+K^{* 2}-2 K^{*} \cos \phi\right)}{U^{* 3}},
$$

The maximum efficiency occurs again at twice the critical velocity when the system starts galloping $\left(U_{\eta_{\max }^{* D M}}^{* D}=2 U_{g}^{* D M}\right)$. Introducing the definition of critical velocity into Eq. 4.101, it yields that the maximum efficiency that can be obtained is

$$
\eta_{\max }^{D M}=-\frac{a_{1}^{2}}{6 a_{3}} \frac{\zeta_{E}}{\zeta_{T}}\left(1+K^{* 2}-2 K^{*} \cos \phi\right) .
$$

The discussion of the maximum harnessable energy is the same as for the $\mathrm{C} 1$ configuration. If there is no parasitic damping $\left(\zeta_{1}=0\right)$, then the maximum efficiency is:

$$
\eta_{\max }^{D M}=\eta_{\max }^{S M}=-\frac{a_{1}^{2}}{6 a_{3}}=\eta_{F}
$$

whereas if $\zeta_{1} \neq 0$, then the maximum harnessable power will be less due to the dissipation of power in the parasitic damping. Dual-mass factors $\left(\psi_{D M}, \chi_{D M}\right)$ relating the maximum efficiency and the critical galloping velocity of the C2 configuration to the Single Mass harvester are also given

$$
\begin{aligned}
& \psi_{D M}=\frac{\gamma_{D M}}{\gamma_{S M}}=\frac{\left(\zeta_{1}+\zeta_{E}\right)\left(1+K^{* 2}-2 K^{*} \cos \phi\right)}{\zeta_{1}+\zeta_{E}\left(1+K^{* 2}-2 K^{*} \cos \phi\right)}, \\
& \chi_{D M}=\frac{U_{g}^{* D M}}{U_{g}^{* S M}}=\frac{\zeta_{1}+\zeta_{E}\left(1+K^{* 2}-2 K^{*} \cos \phi\right)}{\left(\zeta_{1}+\zeta_{E}\right)},
\end{aligned}
$$

and the relationship between $\chi_{D M}$ and $\psi_{D M}$ is

$$
\psi_{D M} \chi_{D M}=1+K^{* 2}-2 K^{*} \cos \phi
$$

Eqs. 4.104 and 4.105 are of the same type as Eqs. 4.89 and 4.91, the only difference is that for the C1 configuration, the trend of $\psi_{D M}$ and $\chi_{D M}$ was given by $K^{*}$, whereas for the $\mathrm{C} 2$ configuration this trend is given now by $1+K^{* 2}-2 K^{* 2} \cos \phi$. If this parameter is bigger than unity, then both $\psi_{D M}$ and $\chi_{D M}$ are larger than unity, otherwise, they will be less than unity. Therefore, the C2 configuration yields the same qualitative behavior as for the C1 configuration. In other words, if $\psi_{D M}^{C 1}=\psi_{D M}^{C 2}$ then $\chi_{D M}^{C 1}=\chi_{D M}^{C 2}$. The main difference is that the previous values are obtained at different parameters of $\mu-\Omega$ at each configuration. The improvement in the $\mathrm{C} 2$ configuration's energy harvesting (as for the C1) comes from the fact that the power dissipated in $\zeta_{E}$ can be enhanced in detriment of $\zeta_{1}$ for correct values of $\mu$ and $\Omega$. In Figs. 4.20 and 4.21, contour plots are given as a function of $\mu$ and $\omega$ for values of $\zeta_{1}=0.0125$ and $\zeta_{E}=0.05$ for $\psi_{D M}$ and $\chi_{D M}$.

\subsubsection{C3 configuration}

The third configuration consists of the same elements as for the previous configurations, except that the generator represented by the damper $c_{E}$ is situated between a fixed wall and the galloping body in parallel to $c_{1}$ (see Fig. 4.14). This configuration is not of practical interest as it only yields similar results to the Single Mass configuration. As studied for the C1 and C2 configurations, the maximum efficiency cannot be enhanced beyond $\eta_{\max }^{D M}=\eta_{F}$, only a distribution of where the energy is dissipated between the parasitic damping or the generator can be obtained, and as $c_{1}$ and $c_{E}$ are situated in parallel, then for all mechanical configurations

$$
\gamma_{D M}=\gamma_{S M}=\frac{\zeta_{E}}{\zeta_{1}+\zeta_{E}} \Rightarrow \psi_{D M}=1
$$


(a)

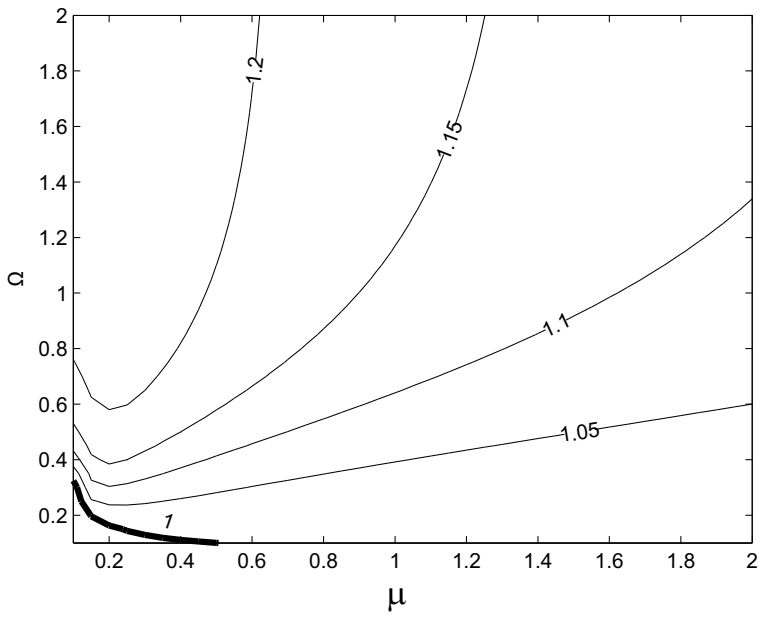

(b)

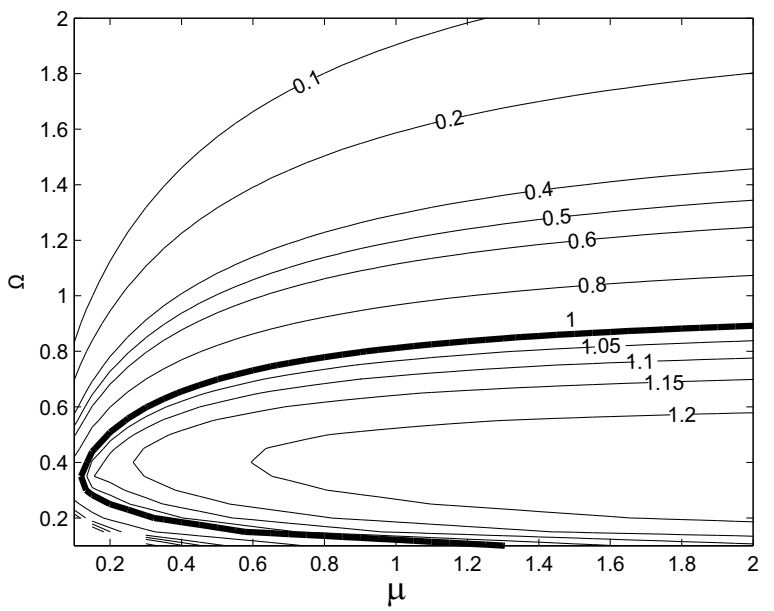

Figure 4.20: (a) Variation of $\psi_{D M}$ with $\Omega$ and $\mu$ for the $\mathrm{C} 2$ configuration ( $\omega^{*}>1$ mode). (b) Variation of $\psi_{D M}$ with $\Omega$ and $\mu$ for the $\mathrm{C} 2$ configuration ( $\omega^{*}<1$ mode).

(a)

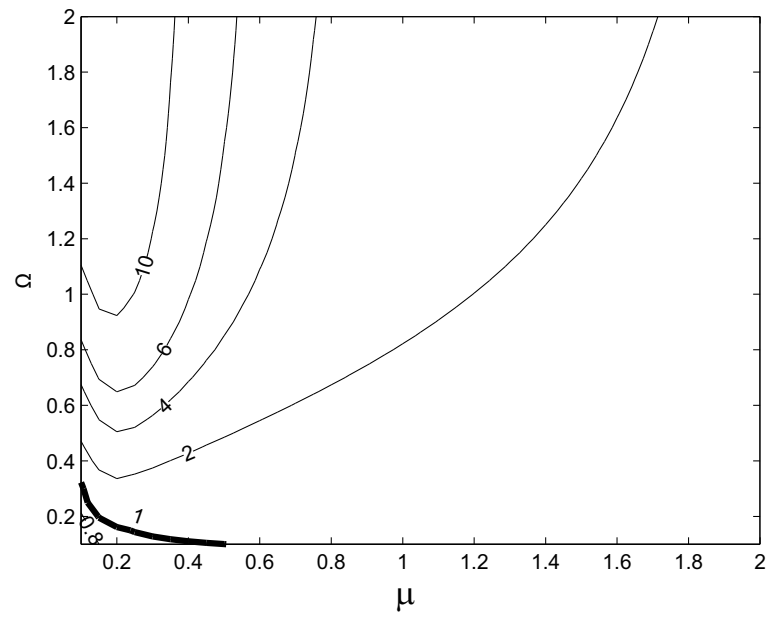

(b)

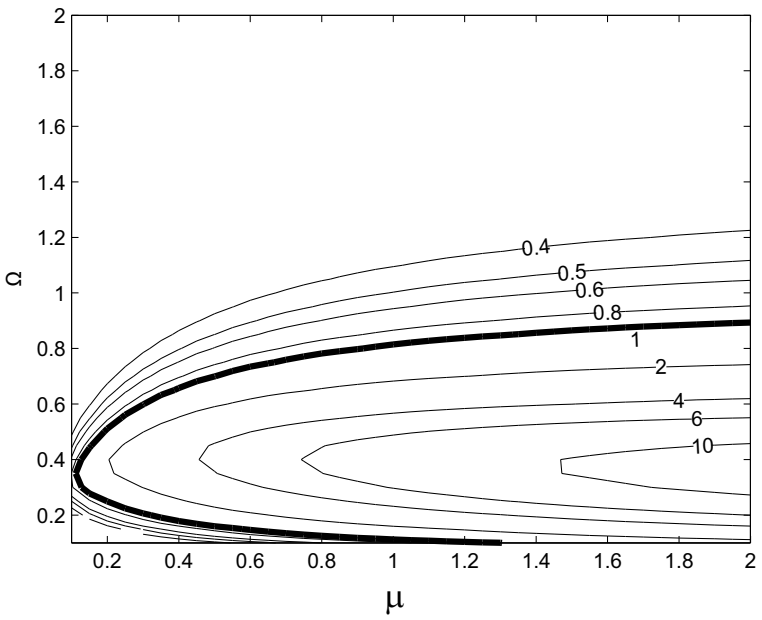

Figure 4.21: Variation of $\chi_{D M}$ with $\Omega$ and $\mu$ for the $\mathrm{C} 2$ configuration ( $\omega^{*}>1$ mode) . (b) Variation of $\chi_{D M}$ with $\Omega$ and $\mu$ for the $\mathrm{C} 2$ configuration ( $\omega^{*}<1$ mode).

$$
U_{g}^{* D M}=U_{g}^{* S M} \Rightarrow \chi_{D M}=1 .
$$

\subsubsection{Concluding remarks}

A dual-mass energy harvester has been considered in order to take advantage of transverse galloping oscillations from a fluid flow. An analytical model has been derived in order to comprehend its potential advantages. The solution exists in a 6 -dimensional space which includes parameters such as the reduced flow velocity $U^{*}$, mass ratio $m^{*}$, parasitic damping $\zeta_{1}$, the harvesting generator $\zeta_{E}$ and two dual mass mechanical parameters, the frequency ratio $\Omega$ and the masses ratio $\mu$. Such a model sheds physical insight to the problem in order to comprehend its potential advantages compared to a single mass case. Some important results have been obtained, the maximum efficiency for each configuration always happens at twice the initial galloping velocity and the maximum efficiency is equivalent to the original single mass system multiplied by a factor that depends solely on $\mu, \Omega$ and $\zeta_{E}$ and a universal curve valid for all dual-mass configurations as well as for the single mass systems has been presented. Three possible configurations have been analyzed obtaining that the maximum 
efficiency cannot surpass that of the single mass system when there is no parasitic damping, but when there is parasitic damping, the efficiency can be enhanced for $\mathrm{C} 1$ and $\mathrm{C} 2$ configurations importantly if the value of the parasitic damping is similar to the harvesting damper. To this end, mechanical parameters of the dual-mass system, namely the frequency ratio $\Omega$ and the masses ratio $\mu$ have to be chosen appropriately. In this sense, a dual-mass system can be considered to be useful to improve the behavior of an already existing installation $\left(m_{1}, k_{1}, c_{1}\right.$ and $\left.c_{E}\right)$ by adding a secondary mass.

An important issue when dealing with real flows, is the variation of the flow velocity and how it affects to the efficiency achievable. In this sense, for the $\mathrm{C} 1$ and $\mathrm{C} 2$ configurations, it has been obtained that it is possible to broaden the values of the incident velocities at which the efficiency has been kept higher, making the efficiency achievable less dependent on flow variations. It must be noted that the analysis carried out is an approximation to the real problem, nonetheless this paper's results are of interest in order to design real devices which have parasitic losses and to extract energy from transverse galloping in an efficient way for real varying flows. 


\subsection{Enhancing mechanical energy extraction by actively ro- tating the galloping body}

Up to now in this chapter, optimal configurations have been studied for energy harvesting from transverse galloping with a focus either on the transduction system (electromagnetic/piezoelectric) or in the elastic system (dual-mass setup). The other place to look for is about the fluid flow around the body; i.e. in the fluid to solid coupling part. According to the quasi-steady theory we can see that a maximum transverse fluid force on the oscillation galloping body is achieved at a certain induced angle of attack (see Fig. 4.2b for different cross-section geometries). From the perspective of energy extraction the most interesting is to have both (i) high $F_{y}$ and (ii) high damping. This suggests the idea to look for the optimal angle of attack at each instant in order to have high $F_{y}$ during most of the time of the oscillation cycle. To this end, let us consider to change continuously the attitude of the body with respect to the incoming cross-flow by actively rotating the body around its axis. In the following, strategies will be proposed to increase the ideal maximum efficiency $\eta_{F}$ that can be harnessed from the current for a given cross-section by modifying the angle of attack appropriately. As will be shown, by doing so it is possible to modify the wake around the galloping prism in such a way that the transverse force coefficient in phase with the velocity on the galloping body is increased. To validate the analytical method that will be proposed, results obtained through the quasi-steady model will be compared to numerical simulations carried out by LBM computations (see Chapter 2 for further details on the Lattice Boltzmann Method employed). It is important to name here the work by Kimsey and Dumas (2008) where the feasibility of extracting energy from a fluid flow using a pitching and heaving airfoil was explored.

\subsubsection{Mathematical model}

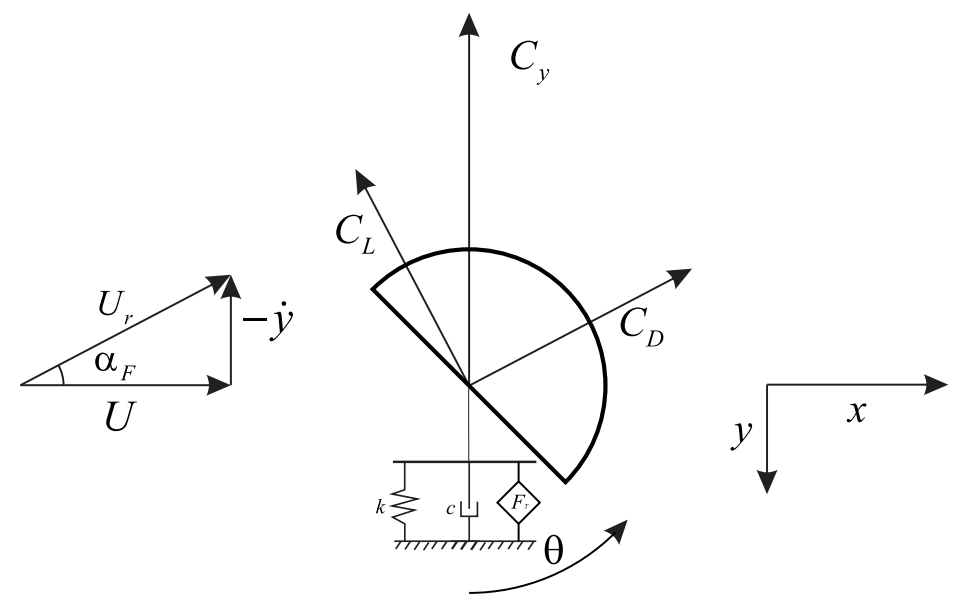

Figure 4.22: Schematics of the forces acting on the galloping prism in a quasi-steady situation.

The simplified dynamical system presented in Section 4.2, which consists of a spring-mounted prismatic body, prone to galloping, in an incoming flow is now actively forced to rotate along its axis with any specified rotation law (see Fig. 4.22). If it can be considered that the rate of rotation is much smaller than the characteristic timescale of convection, $\dot{\theta} \ll U / D$, then the fluid-dynamic force can be evaluated through the quasi-steady model previously presented. For simplicity (and to highlight the effect of actively rotating the prism), a generic viscous damper will be considered as a mean to extract useful power from the current $\left(F_{T}=c \dot{y}\right)$. For this case, the dynamical equation that models 
this configuration in non-dimensional variables can be expressed as

$$
Y^{\prime \prime}+2 \zeta Y^{\prime}+Y=\frac{U^{* 2}}{2 m^{*}} \tilde{C}_{y}
$$

where $\tilde{C}_{y}$ is the modified fluid-dynamic force (due to the rotation of the prism). $\tilde{C}_{y}$ (through a quasisteady analysis) can be determined through the drag $C_{D}$ and lift $C_{L}$ coefficients from static tests as

$$
\begin{aligned}
\tilde{C}_{y} & =\frac{U_{r}}{U}\left(-C_{L}\left(\alpha_{F}+\theta\right) \cos \left(\alpha_{F}\right)-C_{D}\left(\alpha_{F}+\theta\right) \sin \left(\alpha_{F}\right)\right) \\
& =-\frac{C_{L}\left(\alpha_{F}+\theta\right)}{\cos \left(\alpha_{F}\right)}-C_{D}\left(\alpha_{F}+\theta\right) \frac{\tan \left(\alpha_{F}\right)}{\cos \left(\alpha_{F}\right)}
\end{aligned}
$$

where $\alpha_{F}$ is the instantaneous angle of attack, $\tan \left(\alpha_{F}\right)=-Y^{\prime} / U^{*}, \cos \left(\alpha_{F}\right)=U^{*} / U_{r}^{*}, U_{r}^{*}$ is the relative incident flow speed $\left(U_{r}^{*}=\sqrt{U^{*}+Y^{\prime 2}}\right), \theta$ is the rotation applied to the galloping prism and $C_{L}$ and $C_{D}$ are, respectively, the lift and drag fluid force coefficients, which can be obtained through static experiments or numerical simulations as a function of the angle of attack. Hence, $\tilde{C}_{y}$ is mainly a function of the angle of attack $\alpha_{F}$, the rotation applied to the prism $\theta$ and the Reynolds number and cross-section of the galloping prism (through $C_{D}$ and $C_{L}$ ).

To validate the model proposed, as well as to obtain some specific conclusions, a D-section at Reynolds number 100 will be evaluated in the following. Fig. 4.23 shows the dependence of the lift and drag coefficients with the angle of attack $\alpha_{F}$ from numerical simulations carried via LBM computations of a D-section at $R e=100$ (see Chapter 2 for more information about the LBM method used). Also, results from Bhinder et al. (2012) are presented in order to compare and validate present results with results in the literature available.

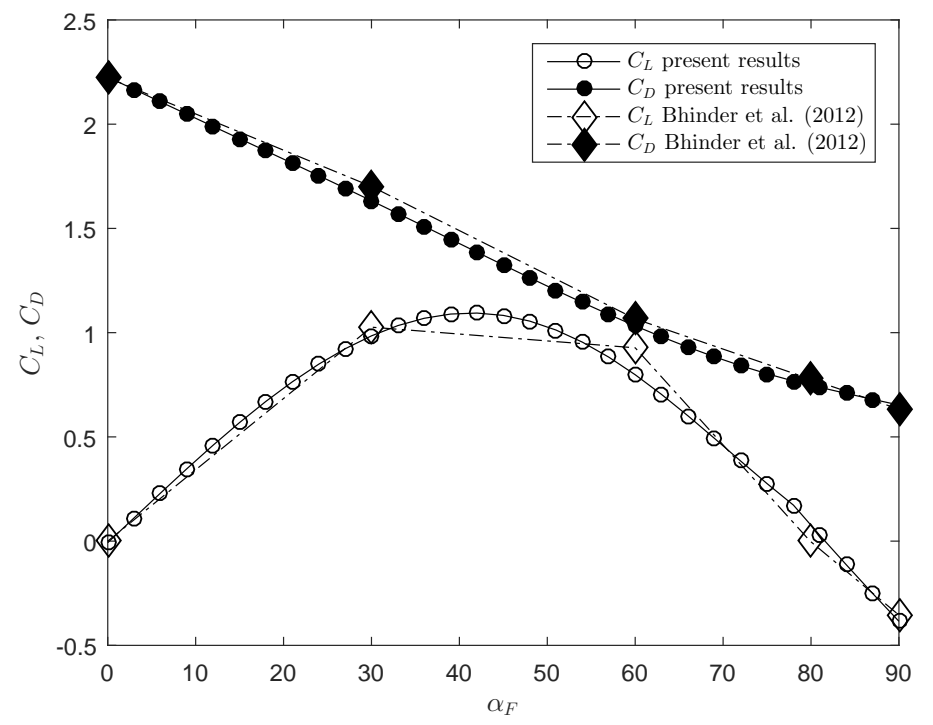

Figure 4.23: $C_{D}$ and $C_{L}$ static static coefficients dependent on the angle of attack computed through numerical LBM computations for a D-section at $R e=100$. For comparison purposes, results from Bhinder et al. (2012) are presented.

In general, with the model proposed, any rotation law ( $\theta$ being a function of the time $)$ that complies with the quasi-steady hypothesis (namely, $\dot{\theta} \ll U / D$ ) can be evaluated analytically with a simple mathematical treatment. Therefore, many rotation laws can be analytically investigated with the purpose of finding those that lead to a significant increase of the maximum efficiency of energy 
harvesting with only input data needed from static experiments of $C_{D}$ and $C_{L}$ for the cross-section under consideration.

For illustration purposes, two rotation laws will be presented and investigated. First, the prism is rotated proportionally to the angle of attack $\alpha_{F}$, and secondly, the prism is rotated proportionally to the displacement of the prism $Y$.

\subsubsection{Rotation of the prism proportional to the angle of attack $\alpha_{F}$}

The study of the transverse galloping phenomenon with actively rotation of the prism proportional to $\alpha_{F}$ will be done in two steps. In first place, the case where the prism is rotated as

$$
\theta=K_{1} \alpha_{F}=K_{1} \tan ^{-1}\left(\frac{Y^{\prime}}{U^{*}}\right),
$$

will be presented in order to discuss the main properties that can be derived; $K_{1}$ is a constant of proportionality. After this rotation law is investigated, secondly, a generalization of Eq. 4.110 will be discussed in order to investigate if there are other rotating laws that can be more interesting and to find rotating laws with higher efficiency.

Introducing the rotation law considered (Eq. 4.110) into Eq. 4.109, then, the modified fluid transverse force coefficient can be expressed as

$$
\tilde{C}_{y}=-\frac{C_{L}\left(\alpha_{F}+K_{1} \alpha_{F}\right)}{\cos \left(\alpha_{F}\right)}-C_{D}\left(\alpha_{F}+K_{1} \alpha_{F}\right) \frac{\tan \left(\alpha_{F}\right)}{\cos \left(\alpha_{F}\right)},
$$

thus, the modified transverse force coefficient is only a function of the angle of attack for each value of $K_{1}$. In Fig. 4.24, $\tilde{C}_{y}$ has been plotted as a function of $\alpha_{F}$ for different values of $K_{1}$. Taking into

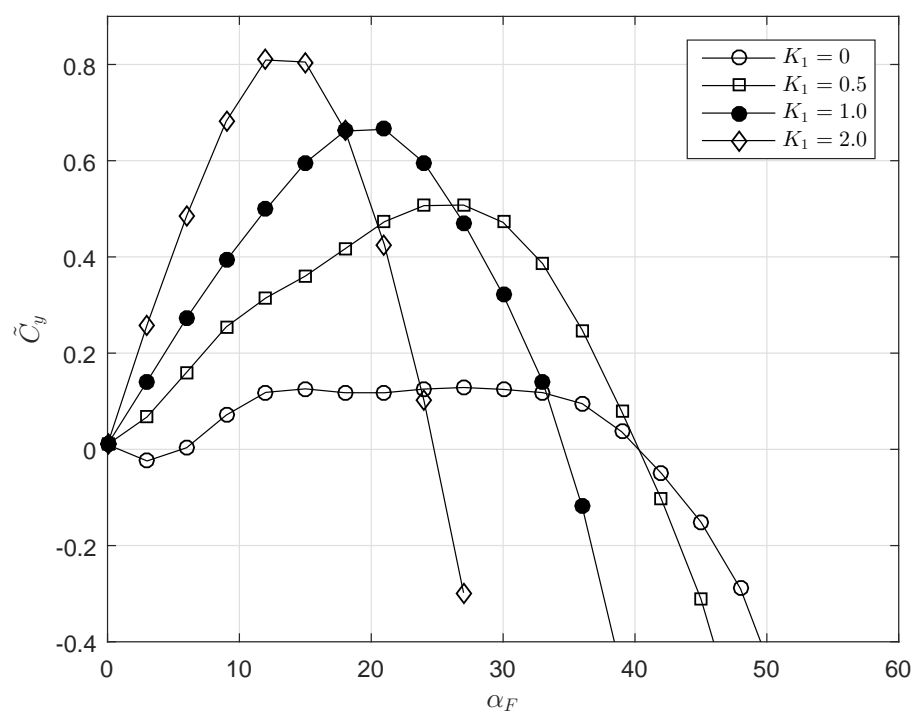

Figure 4.24: Transverse force coefficient $\tilde{C}_{y}$ as a function of $\alpha_{F}$ for different values of $K_{1}$.

account that the $\tilde{C}_{y}$ curves obtained in Fig. 4.24 are for a D-section at Reynolds number 100, it can be seen that as $K_{1}$ is increased, so does the maximum value of $\tilde{C}_{y}$ but such maximum occurs for lower values of $\alpha_{F}$. In addition, increasing $K_{1}$ makes the curve of $\tilde{C}_{y}$ with respect to $\alpha_{F}$ narrower and the cut of the curve with $\tilde{C}_{y}=0$ occurs for lower values of $\alpha_{F}$, except for a small range between $0<K_{1}<0.3$. From Fig. 4.24 , it follows that $\tilde{C}_{y}$ can be approximated by a third order expansion of $\alpha_{F}$. 


$$
\tilde{C}_{y}=\tilde{a}_{1}\left(K_{1}\right) Y^{\prime} / U^{*}+\tilde{a}_{3}\left(K_{1}\right)\left(Y^{\prime} / U^{*}\right)^{3},
$$

where $\tilde{a}_{1}$ and $\tilde{a}_{3}$ are a function of $K_{1}$ and will be obtained through a best fit through the MoorePenrose pseudo-inverse. In Fig. 4.25, the variation of $\tilde{a}_{1}$ and $\tilde{a}_{3}$ with $K_{1}$ is represented. Interestingly, with the rotation law proposed, $\tilde{a}_{1}$ increases nearly linearly with $K_{1}$ whereas $\tilde{a}_{3}$ diminishes rapidly. Note that for values of $K_{1}<-0.2, \tilde{a}_{1}$ has a negative value, thus, galloping oscillations are not possible.

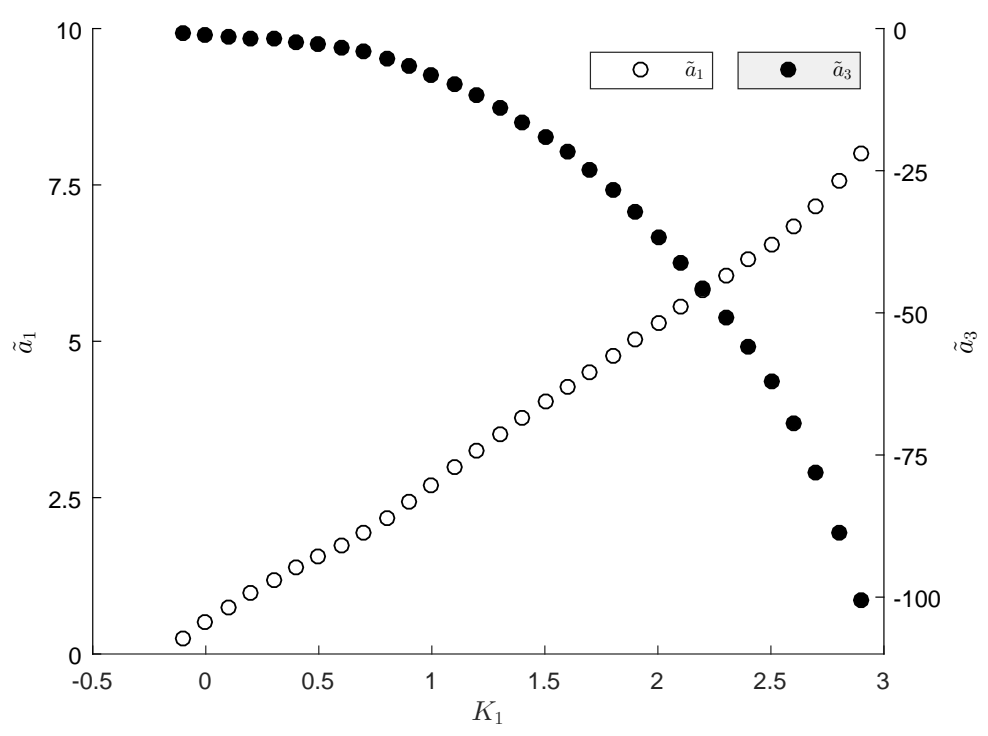

Figure 4.25: Variation of $\tilde{a}_{1}$ and $\tilde{a}_{3}$ as a function of $K_{1}$.

\subsection{Dynamical response and validation}

Solving Eq. 4.108 along with Eq. 4.112 with the Harmonic Balance Method, the dynamical behavior of the prism can be obtained (namely the non-dimensional amplitude $A^{*}$ and the non-dimensional frequency of oscillation $\left.\omega^{*}\right)$ as

$$
\begin{gathered}
A^{*}=\left(\frac{4 U^{*}}{3 \tilde{a}_{3}}\left(4 m^{*} \zeta-\tilde{a}_{1} U^{*}\right)\right)^{1 / 2}, \\
\omega^{*}=1 .
\end{gathered}
$$

As the modified transverse force coefficient is in phase with the velocity, there is no inertia-force term that modifies the frequency of oscillation $\omega^{*}$. Note that $A^{*}$ and $\omega^{*}$ are the same as obtained in Section 4.2, and the only difference is that $\tilde{a}_{1}$ and $\tilde{a}_{3}$ have been modified (as a function of $K_{1}$ ). Oscillations are only possible when the transverse force coefficient overcomes the damping term, thus the initial reduced velocity at which galloping oscillations are expected is recovered

$$
U_{g}^{*}=\frac{4 m^{*} \zeta}{\tilde{a}_{1}},
$$

As $\tilde{a}_{1}$ increases for larger values of $K_{1}$, the initial reduced velocity where galloping starts is reduced as $K_{1}$ is increased.

For validation purposes, in Fig. 4.26 steady-state solution for $A^{*}$ (Eq. 4.113a) against the reduced velocity $U^{*}$ is presented for a D-section prism with reduced mass $m^{*}=10$ for three values of $K_{1}$ and three different values of the damping term $\zeta$. The quasi-steady model presented is capable of 
predicting correctly the amplitude of oscillation as well as the initial galloping reduced velocity $U_{g}^{*}$ for the values of $K_{1}$ and $\zeta$ investigated when compared to the full fluid-structure-interaction numerical simulations conducted via LBM (see Chapter 2).
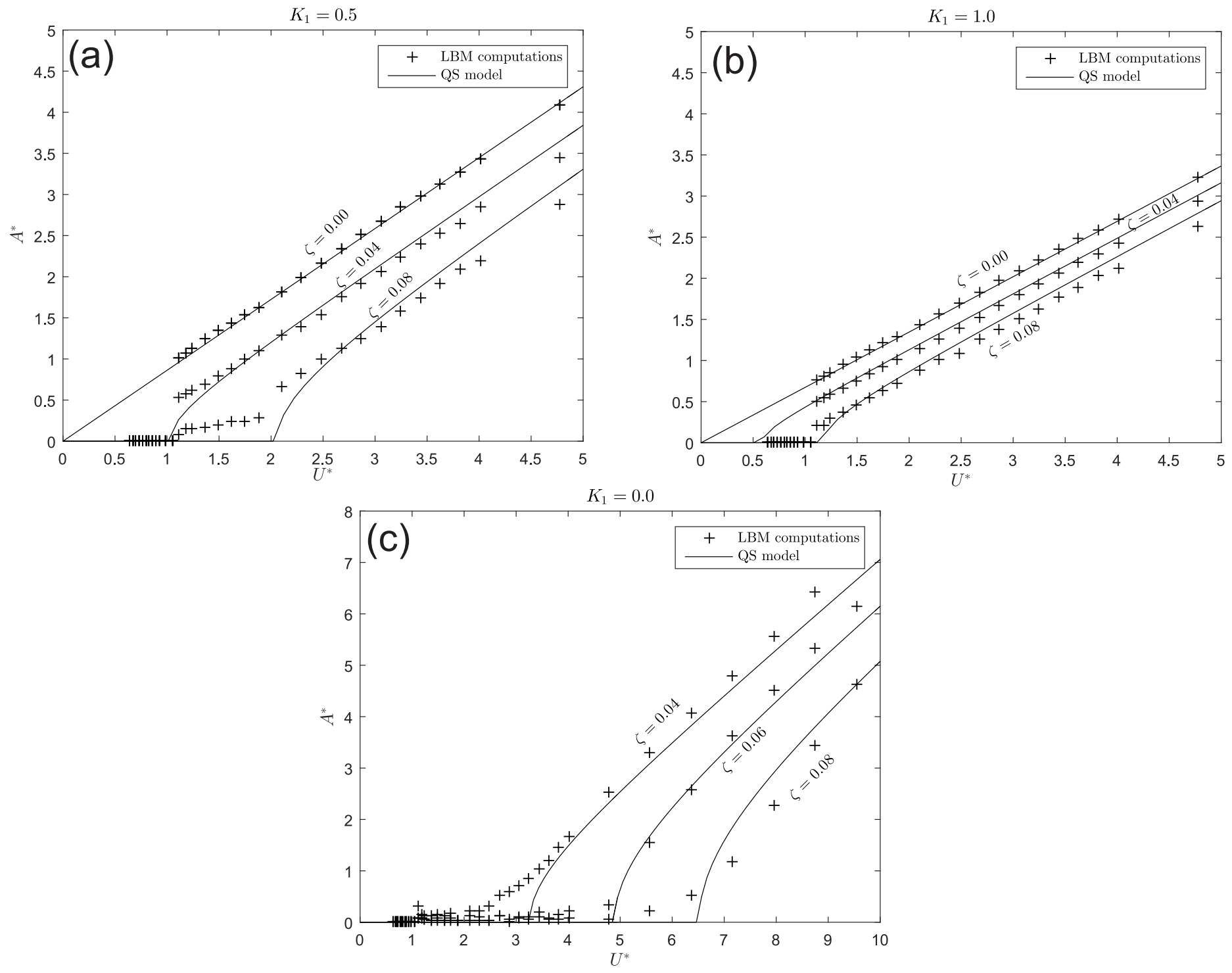

Figure 4.26: Comparison of the amplitude of oscillation $A^{*}$ as a function of the reduced velocity for different values of $K_{1}$ and $\zeta$.

An important parameter is the asymptotic slope of $A^{*}$ for large enough $U^{*}$, obtained by considering $4 m^{*} \zeta$ to be much smaller than $\tilde{a}_{1} U^{*}$ which yields

$$
\frac{A_{\infty}^{*}}{U^{*}}=\sqrt{-\frac{4 \tilde{a}_{1}}{3 \tilde{a}_{3}}}
$$

In Fig. 4.27, the asymptotic slope for $A^{*}$ is plotted as a function of $K_{1}$. For validation purposes, the slope computed from LBM calculations are superimposed. As can be seen, the model is capable of predicting that for small $K_{1}$, the slope increases but right afterwards, the asymptotic slope of the amplitude of oscillation decreases with $K_{1}$. Note that, the asymptotic solution for the slope $A_{\infty}^{*} / U^{*}$ is related to the value of $\alpha_{F}$ where $\tilde{C}_{y}=0$ (from Eq. 4.112 and equating for $\tilde{C}_{y}=0$ ).

In order to compute how a simplified transducer affects the dynamical behavior of the oscillator, 


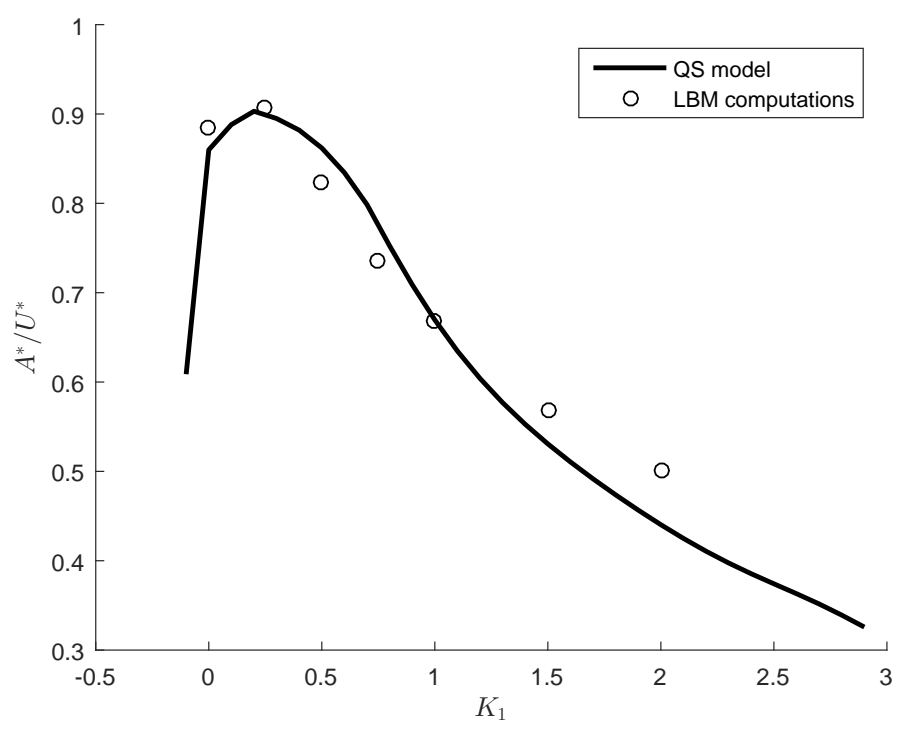

Figure 4.27: Slope of $A^{*}$ for high enough values of $U^{*}$ as a function of $K_{1}$ calculated from the quasisteady model. Superimposed are given the slope obtained through LBM computations.

let us calculate the variation of the amplitude of oscillation with respect to $\zeta$

$$
\frac{\partial A^{*}}{\partial \zeta}=\frac{8 m^{*}}{\sqrt{-3 \tilde{a}_{1} \tilde{a}_{3}}},
$$

which is valid for $\tilde{a}_{1} U^{*} \gg 4 m^{*} \zeta$. Thus, as $K_{1}$ grows, both $\tilde{a}_{1}$ and $\tilde{a}_{3}$ increase (in absolute value) and then, the dependence of the amplitude of oscillation with the damping term is reduced. Interestingly, this dependence is related to the maximum value of $\tilde{C}_{y}$ (see Eq. 4.112), which grows, as seen in Fig. 4.24, when $K_{1}$ is increased. This can also be observed in Fig. 4.26, where the separation between the asymptotic solution is lesser for $K_{1}=1.0$.

\subsection{Energy harvesting efficiency}

As a first approximation it has been considered that the losses due to the power spent to rotate the prism are negligible. The power applied to rotate the prism is

$$
P_{T}=\frac{1}{T} \int_{0}^{T}\left(T_{I}+T_{F}\right) \dot{\theta} d t
$$

where $T_{I}$ refers to the inertial torque and $T_{F}$ is the torque that appears due to the fluid momentum that appears due to the action of the cross-flow. $T_{I}$ is proportional to $\ddot{\theta}$, thus for close-to-sinusoidal actuation, the contribution to Eq. 4.117 is negligible. Also, $T_{F}$ in a quasi-steady manner is proportional to $\theta$, hence, for near sinusoidal rotations, this term can also be neglected resulting in an overall null power consumption.

With respect to the efficiency of energy harvesting, defined equivalently to Section 4.2, Eq. 4.9, one obtains

$$
\eta_{E}=\frac{8 m^{*} \zeta}{3 \tilde{a}_{3} U^{* 2}}\left(4 m^{*} \zeta-\tilde{a}_{1} U^{*}\right),
$$

which is the same topological result as discussed in Section 4.2 , only $\tilde{a}_{1}$ and $\tilde{a}_{3}$ are modified. The 
maximum efficiency (obtained by differentiating Eq. 4.118 with respect to $U^{*}$ and equating to zero) is

$$
\eta_{F}=-\frac{\tilde{a}_{1}^{2}}{6 \tilde{a}_{3}},
$$

which happens at a reduced velocity of $U^{*}=2 U_{g}^{*}$. Note that $\eta_{F}$ is related to the area underneath the force coefficient $\tilde{C}_{y}$ (integrating Eq. 4.112). Thus, in order to maximize the energy harvesting efficiency, it is necessary to look for the rotating law which maximizes the area under $\tilde{C}_{y}$ curve for a given crosssection. For the case in question, Fig. 4.28 plots the maximum efficiency as a function of $K_{1}$ (with superimposed solutions from LBM computations). As seen, for $K_{1}=0$, galloping solution without rotation, the maximum efficiency is approximately $\eta_{F}\left(K_{1}=0\right)=0.042$. As $K_{1}$ is augmented, the efficiency increases rapidly with $K_{1}$ until a maximum efficiency of $\eta_{F}=0.155$ is obtained at $K_{1}=0.8$. Further increasing $K_{1}$ leads to a slow reduction of the maximum efficiency achievable. Through the rotation law investigated (and for a D-section at Reynolds 100), it is possible to increase the maximum achievable efficiency by a factor of approximately 3.7 times. Noteworthy, the low efficiency for $K_{1}=0.0$ (compared to the efficiency described for the same cross-section evaluated in Section 4.2) is mainly due to the Reynolds number (as it has a major effect on the detachment of the flow) and thus, as seen in Fig. 4.1 the transverse force coefficient for the D-section at higher Reynolds number is larger than for the case under consideration (for $K_{1}=0$ ). In Table 4.1, the maximum achievable efficiency for a D-section at Reynolds number $10^{5}$ is $\eta_{F}=0.54$ from experimental tests carried by Novak and Tanaka (1974). ${ }^{4}$

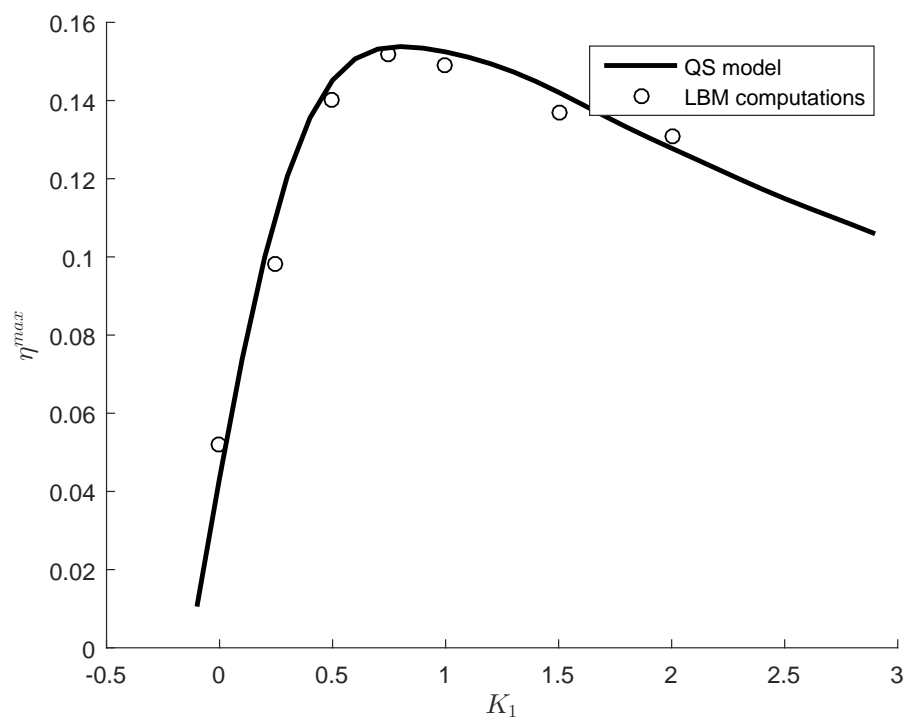

Figure 4.28: Maximum efficiency of energy harvesting as a function of $K_{1}$.

\subsubsection{Generalization $\theta=K_{1}\left(\alpha_{F}\right)^{n}$}

A generalization for the previous rotation law is presented for completeness. In this case, the cylinder is rotated as

$$
\theta=K_{1}\left(\alpha_{F}\right)^{n}
$$

\footnotetext{
${ }^{4}$ Experiments have been carried out with the current rotating law in the recirculating hydrodynamic channel (see Chapter 3 for a detailed description of the setup) for a C-section at $R e \approx 10000$, which yielded an increase in the efficiency of energy extraction from $\eta_{F}\left(K_{1}=0.0\right)=0.6$ to a maximum value of $\eta_{F}=1.2$ at $K_{1}=0.5$ which leaded an increase in efficiency of a factor of 2 .
} 
where $K_{1}$ and $n$ are constants to be varied. Previous discussion is valid, however, as $n$ is modified, the transverse force coefficient changes, inducing changes in $\tilde{a}_{1}$ and $\tilde{a}_{3}$.

Fig. 4.29, represents the asymptotic slope for $A^{*}$ as a function of $K_{1}$ for different values of $n$. For $n<1.0$, the asymptotic slope of $A^{*}$ diminishes more pronouncedly with $K_{1}$ while on the other hand, for $n>1.0$, the asymptotic slope of $A^{*}$ with $K_{1}$ is less dependent and diminishes with $K_{1}$ slower.

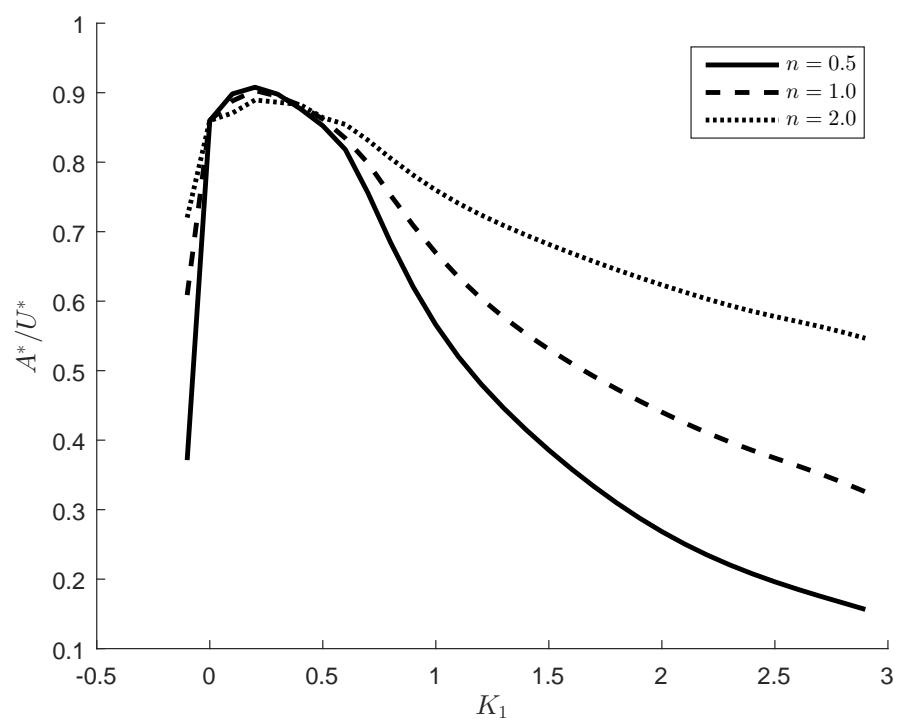

Figure 4.29: Slope of $A^{*}$ for high enough values of $U^{*}$ as a function of $K_{1}$ for different values of $n$.

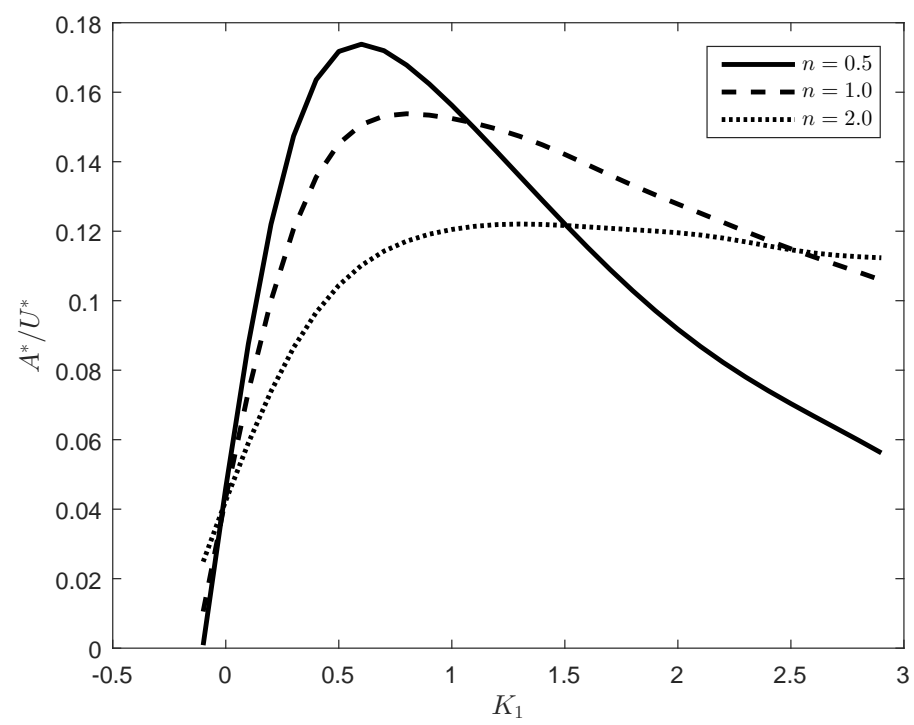

Figure 4.30: Maximum efficiency of energy harvesting as a function of $K_{1}$ for different values of $n$.

In Fig. 4.30 the energy harvesting efficiency $\eta_{F}$ is plotted as a function of $K_{1}$ for different values of $n$. For the values of $n$ investigated, maximum value of $\eta_{F}$ is increased for lower values of $n$, in particular, $\eta_{F}(n=0.5)=0.175$ for $n=0.5$ and $K_{1}=0.6$ but it decreases with increasing $K_{1}$ faster. Also, the value of $K_{1}$ at which the maximum efficiency is obtained is lower. On the other hand, for $n>1.0, \eta_{F}$ is lowered $\eta_{F}(n=2.0)=0.12$ for a value of $K_{1}=1.2$, but it is much less dependent on $K_{1}$ (more broad-band) and the value of $K_{1}$ at which the maximum efficiency is achieved is larger. As expected, efficiency varies importantly depending on the values of $K_{1}$ and $n$ chosen, and results 
should be further validated either numerically or experimentally.

\subsubsection{Comparison of the wake after the galloping body}

In the following, for the sake of completeness, the wake around the galloping body will be presented for the case without actuation $\left(K_{1}=0\right)$ and for a particular case with actuation $\left(K_{1}=1\right.$ and $\left.n=1\right)$ for a D-section at Reynolds 100 for $\zeta=0.04$ at a reduced velocity of $U^{*}=4$.

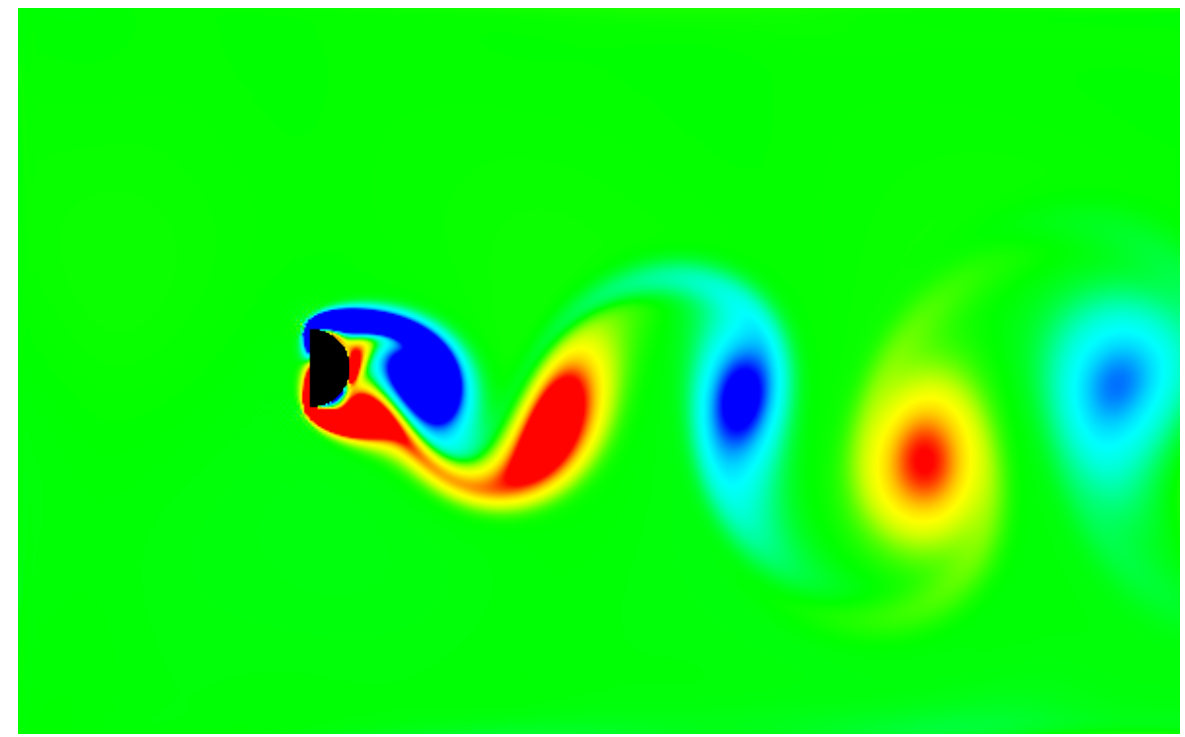

Figure 4.31: Vorticity contour for a D-section at $R e=100$ without actuation for a reduced velocity of $U^{*}=4$, a reduced mass $m^{*}=10$ and a non-dimensional damping parameter of $\zeta=0.04$.

Noticeable, in Fig. 4.31 the wake structure after the body without actuation exhibits characteristic vortices being shed independently from the body's oscillation at its own frequency. Thus, the wake pattern resembles that of a wave as each vortex is shed at different points of the cycle of oscillation following the Strouhal frequency of vortex emission $\left(f_{S t} / f_{N}=\operatorname{St} U^{*}\right)$ for the body at rest, thus, there is no synchronization between vortex emission and oscillation.

On the other hand, in Fig. 4.32 the wake after the body with actuation $\left(K_{1}=1\right.$ and $\left.n=1\right)$ does not exhibit strong vortices being shed. Only independent vortices are formed at its maximum amplitude when the body changes direction (maximum acceleration). Through orientating the body conveniently, the wake behind the body shows bounded vorticity which only brakes into an independent vortex downstream. This could be related to the fact that through orientating the galloping body, it becomes much less bluff and the flow remains further attached.

\subsubsection{Rotation of the prism proportional to the displacement $\theta=K_{2} Y / U^{*}$}

Many other rotation laws are possible and could be evaluated in a similar manner. In particular, it has been chosen to rotate the galloping prism proportionally to the displacement of the prism since passive means can easily be implemented to obtain the desired rotation.

$$
\theta=K_{2} \frac{Y}{U^{*}} .
$$

Particularizing Eq. 4.121 into Eq. 4.109, the transverse force coefficient $\tilde{C}_{y}$ can be expressed as

$$
\tilde{C}_{y}=-\frac{C_{L}\left(\alpha_{F}+K_{2} Y / U^{*}\right)}{\cos \left(\alpha_{F}\right)}-C_{D}\left(\alpha_{F}+K_{2} Y / U^{*}\right) \frac{\tan \left(\alpha_{F}\right)}{\cos \left(\alpha_{F}\right)} .
$$




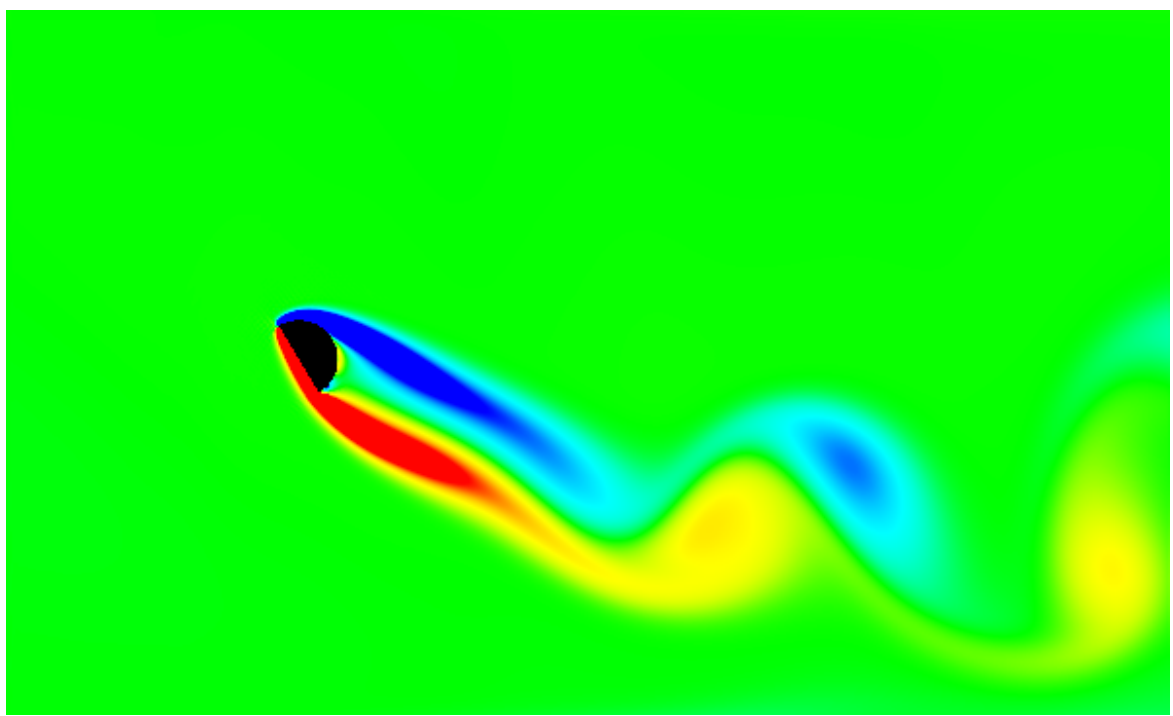

Figure 4.32: Vorticity contour for a D-section at $R e=100$ with actuation $\left(K_{1}=1, n=1\right)$ for a reduced velocity of $U^{*}=4$, a reduced mass $m^{*}=10$ and a non-dimensional damping parameter of $\zeta=0.04$.

The transverse coefficient under evaluation presents terms that are in phase with the position of displacement $Y$ (stiffness effect) and with the velocity of displacement $Y^{\prime}$ (excitation/damping effect). In first place, it is going to be assumed that the transverse coefficient can be written as a series expansion of the type

$$
\tilde{C}_{y}=\tilde{a}_{1} \frac{Y^{\prime}}{U^{*}}+\tilde{a}_{3}\left(\frac{Y^{\prime}}{U^{*}}\right)^{3}+\tilde{b}_{1} \frac{Y}{U^{*}}+\tilde{b}_{3}\left(\frac{Y}{U^{*}}\right)^{3} .
$$

In Fig. 4.33, the coefficients $\tilde{a}_{1}, \tilde{a}_{3}, \tilde{b}_{1}$ and $\tilde{b}_{3}$ have been plotted as a function of $K_{2}$. As can be seen, coefficients in phase with the velocity of oscillation $\left(\tilde{a}_{1}\right.$ and $\left.\tilde{a}_{3}\right)$ are symmetrical with respect to $K_{2}$ and their value is reduced as $K_{2}$ is increased. With regard to the terms of the transverse force coefficient that are in phase with the displacement (which will lead necessarily to a variation in the frequency of oscillation), note that the terms $\tilde{b}_{1}$ and $\tilde{b}_{3}$ are asymmetrical. From Fig. 4.33b, positive values of $K_{2}$ will lead to a reduction in $\omega^{*}$, whereas negative values of $K_{2}$ induces an increase in $\omega^{*}$.
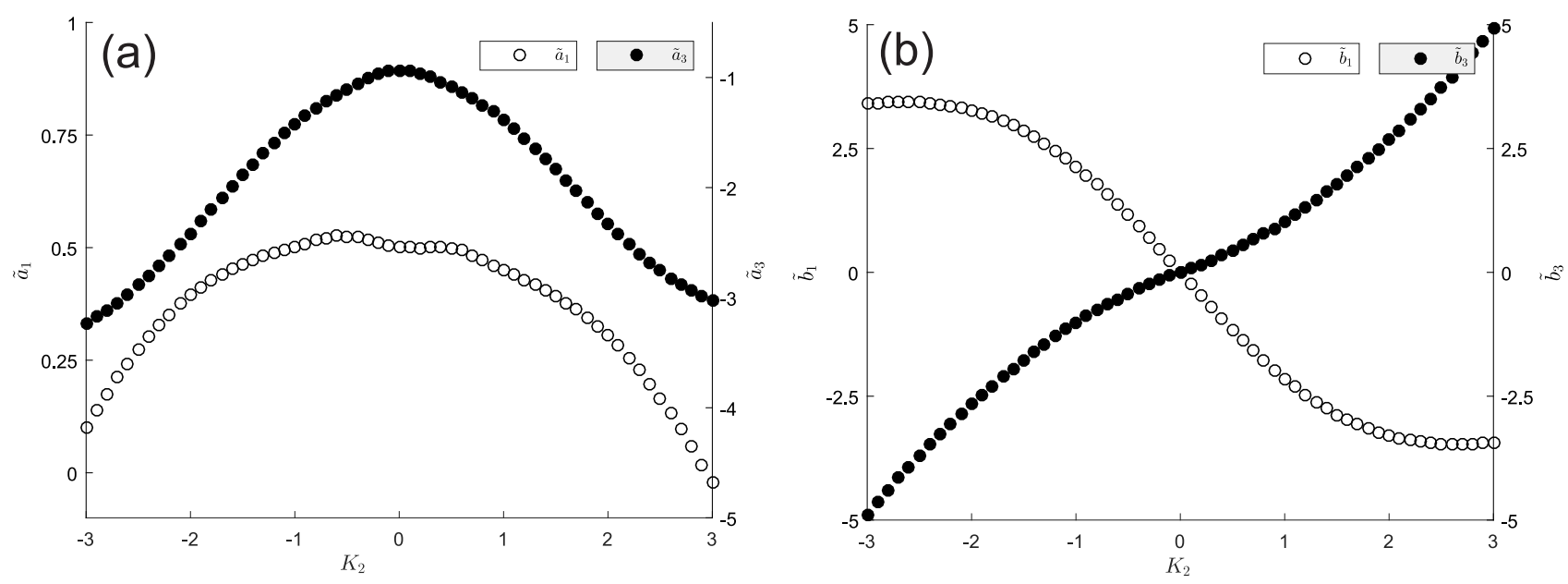

Figure 4.33: Variation of $\tilde{a}_{1}, \tilde{a}_{3}, \tilde{b}_{1}$ and $\tilde{b}_{3}$ as a function of $K_{2}$.

From the dynamical equation (Eq. 4.108) and the transverse force coefficient (Eq. 4.123), one 
obtains after applying HBM

$$
\begin{aligned}
& A^{*} \omega^{*}=\left(\frac{4 U^{*}}{3 \tilde{a}_{3}}\left(4 m^{*} \zeta-\tilde{a}_{1} U^{*}\right)\right)^{1 / 2}, \\
& \omega^{* 2}-1=\frac{U^{* 2}}{2 m^{*}}\left(\tilde{b}_{1} \frac{1}{U^{*}}+\frac{3}{4} \tilde{b}_{3} \frac{A^{* 2}}{U^{* 3}}\right),
\end{aligned}
$$

Eqs. 4.124a and 4.124b have to be solved to obtain the amplitude of oscillation $A^{*}$ and frequency of oscillation $\omega^{*}$, which is not the scope of this article, where the maximization of the energy harvesting is sought. However, some information can be extracted. In particular, the critical velocity where galloping oscillations occur is $U_{g}^{*}=4 m^{*} \zeta / \tilde{a}_{1}$, thus, as $K_{2}$ is increased, it induces that $U_{g}^{*}$ increases, therefore, requiring higher velocity in order to obtain galloping oscillations. Also, generally speaking, amplitude of oscillations is reduced importantly as $\tilde{a}_{1}$ is reduced and the absolute value of $\tilde{a}_{3}$ is increased. Finally, only harmonic solutions will be expected for those values of $K_{2}$ which yield real values of $\omega^{*}$. If only linear terms are considered, the non-dimensional frequency of oscillation can be obtained from Eq. $4.124 \mathrm{~b}$ as $\omega^{* 2}=1+\frac{U^{*}}{2 m^{*}} \tilde{b}_{1}$. If $\tilde{b}_{1}<-\frac{2 m^{*}}{U^{*}}$ (negative values of $\tilde{b}_{1}$ are obtained for positive values of $K_{2}$, see Fig. 4.33), then imaginary solutions for the frequency of oscillation are obtained leading to a divergence-type of solution instead of harmonic oscillations.

However, in order to determine the efficiency of energy harvesting (for harmonic solutions), only Eq. 4.124a is necessary as the efficiency is a function of the product of the amplitude and frequency of oscillation (Eq. 4.118). Note that efficiency of power extraction is only dependent on $\tilde{a}_{1}$ and $\tilde{a}_{3}$ (as for the case when the rotation is proportional to $\alpha_{F}$ ), therefore Eq. 4.119 is applicable. In Fig. 4.34,

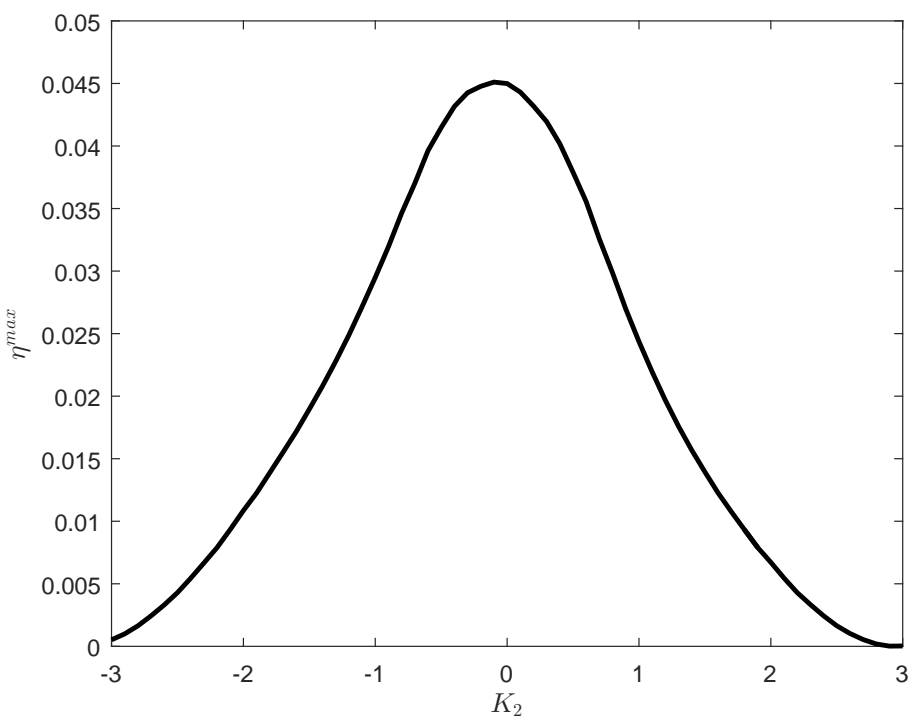

Figure 4.34: Maximum efficiency of energy harvesting as a function of $K_{1}$ for different values of $n$.

the maximum efficiency $\eta_{F}$ is plotted as a function of $K_{2}$. As can be seen, the maximum value of $\eta_{F}$ happens for $K_{2}=0$, and the efficiency diminishes rapidly for positive (and negative values) of $K_{2}$. Thus, this method of rotating the cylinder is not convenient from the point of increasing the efficiency of extraction and should only be considered in cases where a variation of the frequency of oscillation is needed or in order to reduce oscillations caused by the galloping phenomenon (even galloping oscillations can be avoided if $\tilde{a}_{1}$ is reduced enough that the critical velocity $U_{g}^{*}$ is not reached) with a passive method. 


\subsubsection{Concluding remarks}

Inspired by the idea to follow continuously the angle of attack at which quasi-setady transverse fluid force is maximum it is proposed to rotate the galloping body actively. A quasi-steady analysis to evaluate the dynamical behavior and the maximum efficiency of energy harvesting from transverse galloping with active pitch control has been analyzed and presented. The method proposed requires that the characteristic timescale of oscillation $\omega_{N}^{-1}$ and of rotation $\dot{\theta}^{-1}$ has to be much larger than the characteristic timescale of convection $D / U$. The present model is capable of correctly predicting the dynamics of oscillation as well as the energy extracted from the flow with good results and a validation has been presented through LBM computations with the full fluid-solid-interaction simulated for a D-section at Reynolds number 100. With the analytical model proposed it is possible to examine in a simple manner many different rotating strategies. In particular, results have been shown for two strategies:

- Rotation proportional to the angle of attack. A rotation law of the type $\theta=K_{1} \alpha_{F}^{n}$ has been tested. It has been found that it is possible to increase importantly the maximum efficiency of energy harvesting (within the range of parameters tested), in particular, $\eta_{F}$ has been increased for over 4.1 times for $K_{1}=0.6$ and $n=0.5$ with respect without active rotation. Notoriously, the same strategy has been tested experimentally in the water channel inside the GALOPE project (Barrero-Gil et al. UPM P120130288, and collected in patent EP2932091 and extended to China, Canada and USA) for a C-section at $R e \approx 10000$ and the maximum efficiency was increased by 2 times, from $\eta_{F}=0.6$ without actuation up to $\eta_{F}=1.2$ with $n=1$ and $K_{1}=0.5$. In recent years, there have been proposals to increase energy extraction efficiency with large pitch variation of aerodynamic profiles, but pitch control law was not linked with the body's dynamics (position and/or velocity).

- Rotation proportional to the displacement of the prism, $\theta=K_{2} Y / U^{*}$. This method is interesting from the point of view that it can be implemented passively. However, efficiencywise, this strategy reduces the efficiency of energy extraction for all values of $K_{2}$. It also reduces the amplitude of oscillation. On the other hand it increases the critical velocity at which galloping starts. Therefore, it could be considered to reduce the effect of transverse galloping. This strategy induces forces on the prism that are proportional to the displacement $Y$, hence, modifying the frequency of oscillation. 


\section{Chapter 5}

\section{Conclusions and future research}

The present dissertation has been centered on the discussion of the optimization of energy harvesting from two FIV phenomena to gain insight in the role of the different governing parameters. In particular, Vortex-Induced Vibrations and transverse galloping have been taken into consideration as both constitute the basis for the analysis of many related problems. For each of them original contributions have been made which will be hereinafter enlisted. Broadly speaking, the work has been divided into two major segments:

- In Chapters 2 and 3, the possibility of enhancing (or reducing) amplitude of oscillations of a spring-mounted circular cylinder undergoing VIV oscillations with rotation actuation around its axis proportionally to its own dynamics (namely, transverse displacement and velocity) has been investigated. To our knowledge, this is the first time that rotating laws linked to the cylinder's dynamics have been proposed, either for enhancing oscillations of reducing them. The characteristic convective time and oscillation time are of the same order, which results in analytical oversimplified treatments not being reliable to predict the required dynamics. Therefore, the problem has been investigated through numerical simulations and experiments carried out in the free-surface recirculating water channel "Antonio Barrero Ripoll". Numerical simulations of the problem have been carried out implementing a 2D Lattice Boltzmann Method at Reynolds number 100. To date, not many publications exist that use LBM to numerically solve FIV problems so, in some sense, it is of value to develop the code itself and validate it against previous existing numerical approaches for FIV problems.

We believe that LBM method may present very interesting capacities when compared to other numerical schemes. Compared to other traditional approaches, say finite differences, finite element and finite volume, the LBM method presents the following advantages: (i) natural parallel performance. Due to its local nature, in LBM method, each core independently computes the collision step and the moving process of the streaming process, so the parallel performance of LBM method is very good. According to tests performed in our code, it was almost a linear speedup, (ii) simple boundary conditions handling, especially for moving boundaries; (iii) remeshing is not necessary for moving boundaries. The mesh is independent of the body shape and motion.

In Chapter 3, regarding the experimental campaign, all aspects needed to perform FIV experiments in our free-surface water channel have been developed and tuned: namely, water inflow conditions have been taken into account and analyzed, measurement instrumentation has been tested and cross-checked, PIV measurements of the near-wake have been performed, and control instrumentation has been used for real time control of the prism's actuation with minimum lag 
possible. The good agreement of our results of VIV of a circular cylinder with classical data from other leading research groups claim the validity of the installation in consideration.

The main goal was to determine the possibility of enhancing (or reducing) the overall amplitude of oscillation through the strategy proposed, however, an effort has been made to understand the causes of the cylinder's response by analyzing the near-wake through PIV measurements and numerical verification of the fluid flow. Even at this complex flow it has been found that when the characteristic vortex shedding time is larger to the oscillation time (only twice as large) a quasi-steady model is able to capture the main trends of the cylinder's response.

- In Chapter 4, exploiting the disparity of the above commented timescales of the transverse galloping phenomenon allows to introduce simplifying hypothesis which endorses an affordable analytical treatment of the problem. Through this simplified treatment, it has been possible to analyze optimal configurations for the fully-coupled (including electrical generator) energy extraction problem from transverse galloping oscillations. Firstly two models for the transducer have been presented (electromagnetic generator and piezoelectric generator). It has been correctly predicted how different parameters affect the generation of electrical power. Secondly it has been sought if a modified elastic system could help to improve energy extraction by introducing a secondary mass following the early studies by Tang et al. (2011) and Nishi (2013). It has been found that the efficiency is equivalent to the single mass case if no losses as parasitic structural damping is considered. If there is structural parasitic damping, it is possible to obtain mechanical configurations which diminish possible losses in parasitic damping. Finally, inside the quasi-steady framework, it has been considered the rotation of the galloping body in order to increase the maximum available efficiency with different control laws. In particular, proportional to the induced angle of attack where analytical and numerical simulations lead to a significant increase in the efficiency of energy harvesting for a D-section at $R e=100$. No energy input for the rotation law were taken into account.

In coming years, leveraging the numerical capacities developed and experimental facilities available, the knowledge arisen from work at the research group, future short term research efforts should continue to focus on the enhancement of energy harvesting from fluid flows through FIV phenomena. There are several questions that could be addressed, like:

- Non-linear structural dynamics. We, so far, have focused on linear models of the body's mechanism (namely, damping and stiffness), leading to quite periodic oscillatory responses. Numerical simulation or experiments at water channels could be done with non-linear structural dynamics. Complex non-periodic or even chaotic regimes may take place.

Inducing non-linear body dynamics is very easy in numerical simulations. To do that in experiments is more complex, but in our laboratory (not for this thesis) an actuator system able to introduce forces on the body (e.g. the circular cylinder in Chapter 3) in real time has been developed (a time delay correction method was developed to avoid phase lags introduced by the sensor, actuator, filtering the input signals to say a few).

- Non-isolated bodies. In all cases studied, a single isolated body has been considered in an unbounded (or close to unbounded) uniform flow. The behavior in confined flows or with several bodies should be investigated. For example, it could occur that under some cooperative configurations, two bodies extract more energy from a flow than those two same bodies working separately. Some initial experiments have already been carried out, yielding increases of up to $50 \%$ in the amplitude of oscillation of a galloping body situated in certain configurations 
downstream the wake of the primary body (with respect to an equivalent isolated galloping body). In addition, in real environments the incoming flow is expected to be non-uniform and its role should be addressed. In particular, shear layers are not uncommon (in shallow waters) with non-stationary mean velocity of the flow with medium to high levels of turbulence which have big influence on the dynamical response of FIV.

- New experimental and numerical runs. In order to provide a more detailed description of the problems posed at Chapters 2 and 3 it would be convenient to extend parametric space investigated as well as investigating parameters that were left constant (say Reynolds number, mass ratio, damping, reduced velocity, different rotatory laws, geometries different to the circular cylinder, etc.).

To better understand the modes of vortex shedding obtained by actively rotating the circular cylinder it is necessary to study the near wake in more detail, including the study of the stagnation point (which may yield an equivalent angle of attack), the detachment points and their temporal evolution along the wall during the oscillations.

- Unsteady effects on transverse galloping. For low values of the reduced velocity, quasisteady hypothesis might not hold and thus predictions made might be biased. Time-delayed models for the wake and the transverse forces (already developed by the research group) can be introduced into the fully-coupled model of the energy harvesting system in order to enhance the predictive capacities of analytical models proposed for a wider range of reduced velocities as well as reduced masses.

- 3D simulations. The Lattice Boltzmann Method has provided a flexible tool to simulate FIV phenomena (mainly due to easy mesh generation and moving boundaries treatment) providing reliable results compared to existing literature. Nowadays, 3D simulations of FIV phenomena at medium Reynolds numbers are becoming increasingly common. Thus, it would be interesting to increase the capabilities of the software developed to be able to introduce $3 \mathrm{D}$ effects or to simulate a full 3D fluid-solid interaction.

- Transducer models. In the current research, the power has been dissipated on purely resistive electrical resistances. The complexity level in the transducer models may be increased and other ways (like active switching techniques) may be investigated closing the gap with practical engineering applications.

- FIV experiments of a rotating galloping body (C/D-type cross-section). Following the analytical and numerical results presented at the end of Chapter 4, experiments at water channel could be carried out to further study this question. In fact, this work currently under development with promising results.

- Improving PIV resolution. As has been already presented, the temporal and spatial resolution of the PIV system employed was not as good as one may desire in order to obtain reliable quantitative information from the wake around the body in consideration. A method to increase the resolution of the PIV system could be considered by incorporating a hybrid numerical/experimental method. In this case, taking advantage from the local nature of the LBM method, it is possible to feed the numerical method with information coming from PIV measurements of experiments. Then through numerical simulations the flow is propagated until new measurements from PIV are available. Similar attempts have already been made through Reduced Order Models of HOSVD (High Order Singular Value Decomposition) of the PIV measurements (Ma et al., 2003). 


\section{Appendix A}

\section{Effect of cantilevered beams}

The effect of short cantilever beams in the prism dynamics and energy efficiency is studied herein. The main effect is caused by the deflection of the beam at the free end which induces an additional variation of the angle of attack as

$$
\tan \alpha=\frac{Y^{\prime}}{U^{*}}+\delta Y
$$

where $\delta=(3 D) /\left(2 L_{b}\right)$, and the deflection at the free end of the beam is $\theta=(3 y) /(2 L)$ as derived in [22]. It is important to note that this is a mere approximation as further effects derived from the rotation of the beam (such as the effect of the aerodynamic torque) are not considered.

Introducing the modified angle of attack into the aerodynamic force model

$$
c_{y}=a_{1}\left(\frac{\dot{Y}}{U}+\delta Y\right)+a_{3}\left(\frac{\dot{Y}}{U}+\delta Y\right)^{3} \simeq a_{1}\left(\frac{\dot{Y}}{U}+\delta Y\right)+a_{3}\left(\left(\frac{\dot{Y}}{U}\right)^{3}+3 \frac{\dot{Y}}{U} \delta^{2} Y^{2}\right)
$$

in which nonlinear stiffness terms are neglected. Introducing the modified aerodynamic model force into the dynamics equation (Eq. 5a), an analytical solution is obtained valid for small nonlinearities $\left(m^{*} \gg 1\right)$. Operating as in Section 3, the dynamical response of the cantilever beam with an attached galloping prism is

$$
\begin{gathered}
\omega_{\text {cant }}^{* 2}=\frac{-\left(c_{1}-\beta^{2}-2 k_{E} \beta^{2}\right)+\left(\left(c_{1}-\beta^{2}-2 k_{E} \beta^{2}\right)^{2}+4 \beta^{2}\right)^{1 / 2}}{2 \beta^{2}}, \\
A_{\text {cant }}^{* 2}=c_{2} \frac{4 U^{*}}{3 a_{3} \omega_{\text {cant }}^{* 2}}\left(4 m^{*}\left(\zeta+\zeta_{p}\right)-a_{1} U^{*}\right), \\
\varphi_{\text {cant }}=\tan \left(-\beta \omega_{\text {cant }}^{*},\right. \\
\frac{V}{A_{\text {cant }}^{*}}=\frac{2 k_{E} \beta \omega_{\text {cant }}^{*}}{\beta \omega_{\text {cant }}^{*} \sin \varphi_{\text {cant }}-\cos \varphi_{\text {cant }}} .
\end{gathered}
$$

Where "cant" stands for cantilever and $c_{1}$ and $c_{2}$ are correcting terms due to the cantilever effect that take a value of

$$
\begin{gathered}
c_{1}=1+\frac{a_{1} U^{* 2} \delta}{2 m^{*}} \\
c_{2}=\left(1+\frac{a_{1} U^{* 2} \delta}{2 m^{*}}+U^{* 2} \delta^{2}\right)^{-1} .
\end{gathered}
$$

From Eq. A.4, the reduced velocity at which the galloping instability happens is not affected by such modification and therefore $U_{g}^{*}$ is directly obtained from Eq. 15. For long cantilever beams $(\delta \ll 1)$ both factors $\left(c_{1}\right.$ and $\left.c_{2}\right)$ tend to 1 , thus recovering the solution for pure transverse galloping discussed 
in Section 3.

The power extracted (as in Eq. 7) is

$$
P_{E}=\frac{2 V_{c}^{2}}{R_{L}} \frac{k_{E}^{2} \beta^{2}}{1+\omega^{*} 2_{\text {cant }} \beta^{2}} A_{\text {cant }}^{* 2} \omega_{\text {cant }}^{* 2},
$$

and its efficiency, which compares the power output of electricity dissipated in an equivalent electric load to the kinetic power of the airstream in a section of the dimension of the galloping prism is

$$
\eta_{\text {cant }}=c_{2} \frac{8 m^{*} k_{e} \beta}{\left(1+\beta^{2} \omega_{\text {cant }}^{* 2}\right) 3 a_{3} U^{* 2}}\left(4 m^{*}\left(\zeta+\zeta_{p}\right)-a_{1} U^{*}\right) .
$$

The relation between $\eta_{\text {cant }}$ and the efficiency $\eta$ of the pure galloping systems:

$$
\eta_{\text {cant }}=c_{2} \frac{1+\beta^{2} \omega_{\text {cant }}^{* 2}}{1+\beta^{2} \omega^{* 2}} \eta
$$

where it can be observed that $\eta_{c a n} l \eta$ for all conditions. When $U^{* 2} \delta / m^{*} \ll 1$, a typical condition for transverse galloping in an airstream and thus $m^{*} \gg 1, c_{1} \rightarrow 1$ and $c_{2} \rightarrow\left(1+U^{* 2} \delta^{2}\right)^{-1}$ and thus the efficiency can be simplified as $\eta_{\text {cant }}=\eta /\left(1+U^{* 2} \delta^{2}\right)$. The decrease in the efficiency for cantilevered beams only depends on $U^{*} \delta$. On the other hand, the ratio $\eta_{\text {cant }} / \eta$ does not depend on the electrical load $\beta$.

To characterize the efficiency map as a function of $U^{*}$ and $\beta$, a similar study to the one carried out in Section 4 is presented. In first place, the reduced velocity at which the maximum efficiency happens for constant electric load occurs when the condition $\partial \eta_{\text {cant }} / \partial U^{*}=0$ is fulfilled. It is proven analytically that such maximum is dependent on the product $U_{g}^{*} \delta$. In Fig. A.1, the value $U_{\eta^{\max }}^{*}$ at which the maximum efficiency occurs is plotted as a function of $U_{g}^{*} \delta$. If $U_{g}^{*} \delta \ll 1$, then the pure transverse galloping solution is recovered where $U_{\eta^{\max }}^{*}=2 U_{g}^{*}$, whereas if $U_{g}^{*} \delta \gg 1$, the value of the incident velocity at which the maximum efficiency occurs tends asymptotically to $U_{\eta^{\max }}^{*}=4 / 3 U_{g}^{*}$. Thus, for all conditions of the cantilever beam's length, the maximum efficiency occurs for $4 / 3<U_{\eta^{\max }}^{*}(\delta) / U_{g}^{*}<2$. This result is important to design the piezoelectric harvester so as to be close to the maximum efficiency for all conditions.

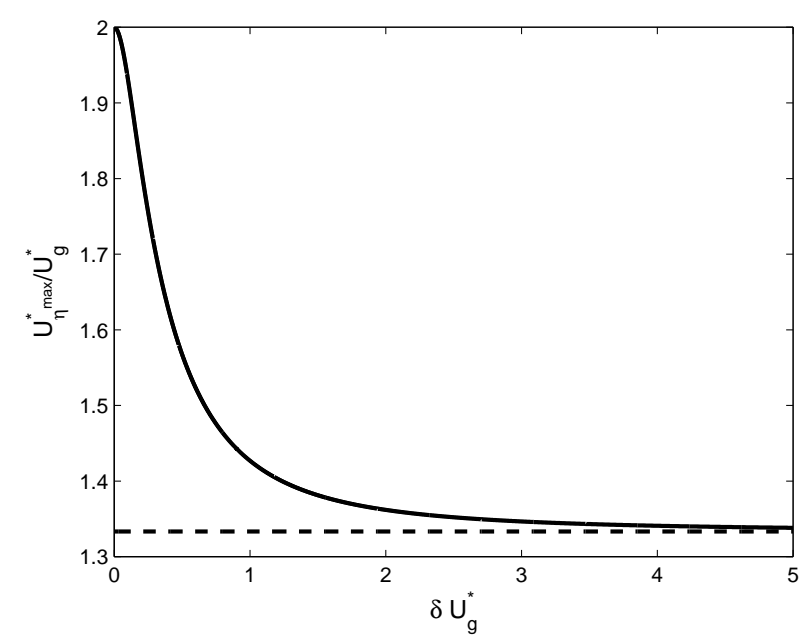

Figure A.1: Reduced velocity at which the maximum efficiency is obtained as a function of $\delta$ times the initial reduced galloping velocity. Dashed line represents the asymptotic solution for $\delta U_{g}^{*} \gg 1$.

With regard to the electric load that maximizes the efficiency for each value of $U^{*}$, it is possible to obtain the law for the optimum electric load by solving $\partial \eta / \partial \beta=0$. Operating in the same way as 
in Section 4, yields

$$
\begin{gathered}
\beta_{o p 1}=\frac{4 m^{*} k_{E}-\left(-U^{* 2} a_{1}^{2} c_{1}+8 U^{*} a_{1} m^{*} \zeta c_{1}+16 k_{E}^{2} m^{* 2}-16 m^{* 2} \zeta^{2} c_{1}\right)^{1 / 2}}{c_{1}\left(U^{*} a_{1}-4 m^{*} \zeta\right)}, \\
\beta_{o p 2}=\frac{4 m^{*} k_{E}+\left(-U^{* 2} a_{1}^{2} c_{1}+8 U^{*} a_{1} m^{*} \zeta c_{1}+16 k_{E}^{2} m^{* 2}-16 m^{* 2} \zeta^{2} c_{1}\right)^{1 / 2}}{c_{1}\left(U^{*} a_{1}-4 m^{*} \zeta\right)}, \\
\beta_{o p 3}=\frac{1}{c 1^{1 / 2}},
\end{gathered}
$$

which is the same result as for the transverse galloping energy harvesting (Eqs. 21) except for the correction introduced by the factor $c_{1}$. If it is considered that $U^{* 2} \delta / m^{*} \ll 1$, then the correcting factor $c_{1} \rightarrow 1$, and therefore the solution is equivalent to that of pure transverse galloping. The reduced incident velocity at which the three different solutions collapse can be obtained by equating $\beta_{o p 1}$ and $\beta_{o p 2}$ as

$$
U_{\text {joint }}^{*}=\frac{4 m^{*}\left(\zeta c_{1}^{1 / 2}+k_{E}\right)}{a_{1} c_{1}^{1 / 2}}
$$




\section{Bibliography}

Abdelkefi, A., Hajj, M. R., Nayfeh, A. H. (2012a). Phenomena and modeling of piezoelectric energy harvesting from freely oscillating cylinders. Nonlinear Dynamics, 70(2), 1377-1388.

Abdelkefi A., Hajj M.R., Nayfeh A.H., (2012b). Power harvesting from transverse galloping of square cylinder, Nonlinear Dynamics 70(2), 1355-1363.

Abdelkefi, A., Scanlon, J. M., McDowell, E., Hajj, M. R. (2013a). Performance enhancement of piezoelectric energy harvesters from wake galloping. Applied Physics Letters, 103(3), 033903.

Abdelkefi, A., Yan, Z., Hajj, M. R. (2013b). Modeling and nonlinear analysis of piezoelectric energy harvesting from transverse galloping. Smart materials and Structures, 22(2), 025016.

Abdelkefi A., Hajj M.R., Nayfeh A.H., (2013c). Piezoelectric energy harvesting from transverse galloping of bluff bodies, Smart Materials and Structures 22(1), 015014.

Adrian, R. J., Westerweel, J. (2011). Particle image velocimetry (No. 30). Cambridge University Press.

Afsharfard, A., Farshidianfar, A. (2014). Application of single unit impact dampers to harvest energy and suppress vibrations. Journal of Intelligent Material Systems and Structures, 1045389X14535012.

Akaydin, H. D., Elvin, N., Andreopoulos, Y. (2012). The performance of a self-excited fluidic energy harvester. Smart Materials and Structures, 21(2), 025007.

Al-Mdallal, Q.M., (2004). Analysis and computation of the cross-flow past an oscillating cylinder with two degrees of freedom. Ph.D.Thesis, Memorial University of Newfoundland.

Allen J.J., Smits A.J., (2001). Energy harvesting eel, Journal of Fluids and Structures 15, 629-640.

Alonso, G., Meseguer, J., Pérez-Grande, I. (2007). Galloping stability of triangular cross-sectional bodies: a systematic approach. Journal of Wind Engineering and Industrial Aerodynamics, 95(9), 928-940.

Assi, G. R. S., Meneghini, J. R., Aranha, J. A. P., Bearman, P. W., Casaprima, E. (2006). Experimental investigation of flow-induced vibration interference between two circular cylinders. Journal of Fluids and Structures, 22(6), 819-827.

Badr, H. M., Coutanceau, M., Dennis, S. C. R., Menard, C. (1990). Unsteady flow past a rotating circular cylinder at Reynolds numbers 103 and 10 4. Journal of Fluid Mechanics, 220, 459-484. 
Bae, J. S., Inman, D. J. (2013). Aeroelastic characteristics of linear and nonlinear piezo-aeroelastic energy harvester. Journal of Intelligent Material Systems and Structures, 1045389X13498312.

Barrero-Gil, A., Sanz-Andrés, A., Alonso, G. (2009). Hysteresis in transverse galloping: the role of the inflection points. Journal of Fluids and Structures, 25(6), 1007-1020.

Barrero-Gil A., Sanz-Andrés A., Alonso G., (2010). Energy Harvesting from Transverse Galloping, Journal of Sound and Vibration 329(14),2873-2883.

Barrero-Gil, A., Pindado, S., Avila, S. (2012). Extracting energy from vortex-induced vibrations: a parametric study. Applied mathematical modelling, 36(7), 3153-3160.

Berkooz, G., Holmes, P., Lumley, J. L. (1993). The proper orthogonal decomposition in the analysis of turbulent flows. Annual review of fluid mechanics, 25(1), 539-575.

Bernitsas M.M., Raghavan K., Ben-Simon Y., Garcia E. M. H., (2008). VIVACE (Vortex Induced Vibration Aquatic Clean Energy): A New Concept in Generation of Clean and Renewable Energy from Fluid, Journal of Offshore Mechanics and Arctic Engineering, ASME Transactions 130(4), 041101-15.

Blackburn, H.M., Elston,J.R., Sheridan,J., (1999). Bluff body propulsion produced by combined rotary and translational oscillation. Physics of Fluids 11 (1), 4-6.

Blevins R., (1990). Flow-induced vibrations, Van Nostrand Reinhold.

Bourguet, R., Jacono, D. L. (2014). Flow-induced vibrations of a rotating cylinder. Journal of Fluid Mechanics, 740, 342-380.

Bradshaw, P., Pankhurst, R. C. (1964). The design of low-speed wind tunnels. Progress in Aerospace Sciences, 5, 1-69.

Brennen, C. E. (1982). A review of added mass and fluid inertial forces.

Brika, D., Laneville, A. (1993). Vortex-induced vibrations of a long flexible circular cylinder. Journal of Fluid Mechanics, 250, 481-481.

Bryant, M., Garcia, E. (2011). Modeling and testing of a novel aeroelastic flutter energy harvester. Journal of vibration and acoustics, 133(1), 011010.

Burton, T., Sharpe, D., Jenkins, N., Bossanyi, E. (2001). Wind energy handbook. John Wiley and Sons.

Caiazzo, A., Junk, M. (2008). Boundary forces in lattice Boltzmann: Analysis of momentum exchange algorithm. Computers and Mathematics with Applications, 55(7), 1415-1423.

Carberry, J., Sheridan, J., Rockwell, D. (2005). Controlled oscillations of a cylinder: forces and wake modes. Journal of Fluid Mechanics, 538, 31-69. 
Chen, S., Doolen, G. D. (1998). Lattice Boltzmann method for fluid flows. Annual review of fluid mechanics, 30(1), 329-364.

De Broe A.M., Drouilhet S., Gevorgian V., 1999. A Peak Power Tracker for Small Wind Turbines in Battery Charging Applications, IEEE Transactions on Energy Conversion, 14, 4, 1630-1635.

De Langre, E. (2008). Effects of wind on plants. Annu. Rev. Fluid Mech., 40, 141-168.

Ding, L., Zhang, L., Wu, C., Mao, X., Jiang, D. (2015). Flow induced motion and energy harvesting of bluff bodies with different cross sections. Energy Conversion and Management, 91, 416-426.

Doaré, O., Michelin, S. (2011). Piezoelectric coupling in energy-harvesting fluttering flexible plates: linear stability analysis and conversion efficiency. Journal of Fluids and Structures, 27(8), 1357-1375.

Dunnmon, J. A., Stanton, S. C., Mann, B. P., Dowell, E. H. (2011). Power extraction from aeroelastic limit cycle oscillations. Journal of Fluids and Structures, 27(8), 1182-1198.

El-Hami, M., Glynne-Jones, P., White, N. M., Hill, M., Beeby, S., James, E., Ross, J. N. (2001). Design and fabrication of a new vibration-based electromechanical power generator. Sensors and Actuators A: Physical, 92(1), 335-342.

Erturk, A. (2009). Electromechanical modeling of piezoelectric energy harvesters (Doctoral dissertation, Virginia Polytechnic Institute and State University).

Gabbai, R. D., Benaroya, H. (2005). An overview of modeling and experiments of vortex-induced vibration of circular cylinders. Journal of Sound and Vibration, 282(3), 575-616.

Govardhan, R., Williamson, C. H. K. (2000). Modes of vortex formation and frequency response of a freely vibrating cylinder. Journal of Fluid Mechanics, 420, 85-130.

Govardhan, R., Williamson, C. H. K. (2002). Resonance forever: existence of a critical mass and an infinite regime of resonance in vortex-induced vibration. Journal of Fluid Mechanics, 473, 147-166.

Govardhan, R. N., Williamson, C. H. K. (2006). Defining the modified Griffin plot in vortexinduced vibration: revealing the effect of Reynolds number using controlled damping. Journal of Fluid Mechanics, 561, 147-180.

Grouthier, C., Michelin, S., de Langre, E. (2013, June). Energy harvesting by vortex-induced vibrations in slender structures. In ASME 2013 32nd International Conference on Ocean, Offshore and Arctic Engineering (pp. V007T08A013-V007T08A013). American Society of Mechanical Engineers.

Higuera, F. J. (1989). Boltzmann approach to lattice gas simulations. EPL (Europhysics Letters), $9(7), 663$.

Inamuro, T., Maeba, K., Ogino, F. (2000). Flow between parallel walls containing the lines of neutrally buoyant circular cylinders. International Journal of Multiphase Flow, 26(12), 1981-2004.

Jiang, X., Andreopoulos, Y., Lee, T., Wang, Z. (2016). Numerical investigations on the vortexinduced vibration of moving square cylinder by using incompressible lattice Boltzmann method. Com- 
puters and Fluids, 124, 270-277.

Jung, H. J., Lee, S. W. (2011). The experimental validation of a new energy harvesting system based on the wake galloping phenomenon. Smart Materials and Structures, 20(5), 055022.

Karabelas, S. J., Koumroglou, B. C., Argyropoulos, C. D., Markatos, N. C. (2012). High Reynolds number turbulent flow past a rotating cylinder. Applied mathematical modelling, 36(1), 379-398.

Khalak, A., Williamson, C. H. K. (1999). Motions, forces and mode transitions in vortex-induced vibrations at low mass-damping. Journal of fluids and Structures, 13(7), 813-851.

Kinsey, T., Dumas, G. (2008). Parametric study of an oscillating airfoil in a power-extraction regime. AIAA journal, 46(6), 1318-1330.

Klamo, J. T. (2007). Effects of damping and Reynolds number on vortex-induced vibrations (Doctoral dissertation, California Institute of Technology).

Kluger, J. M., Moon, F. C., Rand, R. H. (2013). Shape optimization of a blunt body vibro-wind galloping oscillator. Journal of Fluids and Structures, 40, 185-200.

Kocabiyik, S., Al-Mdallal, Q.M., (2005). Bluff-body flow created by combined rotary and translational oscillation. Fluid Structure Interaction and Moving Boundary Problems, vol 84. WIT Press, Southampton, UK, pp.195-203.

Koutroulis E., Kalaitzakis K., (2006). Design of a Maximum Power Tracking System for WindEnergy-Conversion Applications, IEEE Transactions on Industrial Electronics 53, 2, 486-494.

$\mathrm{Ku}$, X., Lin, J. (2005). Numerical simulation of the flows over two tandem cylinders by lattice Boltzmann method. Modern Physics Letters B, 19(28n29), 1551-1554.

Lallemand, P., Luo, L. S. (2003). Lattice Boltzmann method for moving boundaries. Journal of Computational Physics, 184(2), 406-421.

Lee, J. H., Xiros, N., Bernitsas, M. M. (2011). Virtual damper-spring system for VIV experiments and hydrokinetic energy conversion. Ocean Engineering, 38(5), 732-747.

Luo, S. C., Chew, Y. T., Lee, T. S., Yazdani, M. G. (1998). Stability to translational galloping vibration of cylinders at different mean angles of attack. Journal of Sound and Vibration, 215(5), 1183-1194.

Ma, X., Karniadakis, G. E., Park, H., Gharib, M. (2003). DPIV-driven flow simulation: a new computational paradigm. In Proceedings of the Royal Society of London A: Mathematical, Physical and Engineering Sciences (Vol. 459, No. 2031, pp. 547-565). The Royal Society.

Mackowski, A. W., Williamson, C. H. K. (2013). An experimental investigation of vortex-induced vibration with nonlinear restoring forces. Physics of Fluids (1994-present), 25(8), 087101.

Maxey, M. R., Riley, J. J. (1983). Equation of motion for a small rigid sphere in a nonuniform flow. The Physics of Fluids, 26(4), 883-889. 
Mei, R., Luo, L. S., Shyy, W. (1999). An accurate curved boundary treatment in the lattice Boltzmann method. Journal of computational physics, 155(2), 307-330.

Mittal, S., Kumar, B. (2003). Flow past a rotating cylinder. Journal of Fluid Mechanics, 476, 303-334.

Mehmood, A., Abdelkefi, A., Hajj, M. R., Nayfeh, A. H., Akhtar, I., Nuhait, A. O. (2013). Piezoelectric energy harvesting from vortex-induced vibrations of circular cylinder. Journal of Sound and Vibration, 332(19), 4656-4667.

Moor G.D., Beukes H.J., 2004. Maximum Power Point Trackers for Wind Turbines, 35th Annual IEEE Power Electronics Specialists Conference, 2044-2049.

Morse, T. L., Govardhan, R. N., Williamson, C. H. K. (2008). The effect of end conditions on the vortex-induced vibration of cylinders. Journal of Fluids and Structures, 24(8), 1227-1239.

Morse, T. L., Williamson, C. H. K. (2009). Fluid forcing, wake modes, and transitions for a cylinder undergoing controlled oscillations. Journal of Fluids and Structures, 25(4), 697-712.

Mussa, A., Asinari, P., Luo, L. S. (2009). Lattice Boltzmann simulations of 2D laminar flows past two tandem cylinders. Journal of computational physics, 228(4), 983-999.

Naudascher E., Rockwell D, 1994. Flow-Induced Vibrations: An Engineering Guide. A.A. Balkema, Rotterdam.

Nazarinia, M.,Lo Jacono, D., Thompson, M.C., Sheridan, J., (2009a). Flow behind a cylinder forced by a combination of oscillatory translational and rotational motions. Physics of Fluids 21(5), 051701.

Nazarinia, M., Lo Jacono, D., Thompson, M.C., Sheridan, J., (2009b). The three-dimensional wake of a cylinder undergoing a combination of translational and rotational oscillation in a quiescent fluid. Physics of Fluids 21(6),064101.

Nishi, Y. (2013). Power extraction from vortex-induced vibration of dual mass system. Journal of Sound and Vibration, 332(1), 199-212.

Nishi, Y., Ueno, Y., Nishio, M., Quadrante, L. A. R., Kokubun, K. (2014). Power extraction using flow-induced vibration of a circular cylinder placed near another fixed cylinder. Journal of Sound and Vibration, 333(10), 2863-2880.

Novak, M., Tanaka, H. (1974). Effect of turbulence on galloping instability. Journal of the Engineering Mechanics Division, 100(1), 27-47.

Paidoussis M.P., Stuart J.P., DE Langre E., (2011). Fluid-Structure Interactions, Cambridge University Press.

Park, G., Rosing, T., Todd, M. D., Farrar, C. R., Hodgkiss, W. (2008). Energy harvesting for structural health monitoring sensor networks. Journal of Infrastructure Systems, 14(1), 64-79. 
Parkinson G, (1989). Phenomena and modelling of flow-induced vibrations of bluff bodies, Progress in Aerospace Sciences 26 (2), 169-224

Parkinson, G. V., Smith, J. D. (1964). The square prism as an aeroelastic non-linear oscillator. The Quarterly Journal of Mechanics and Applied Mathematics, 17(2), 225-239.

Radi, A., Jacono, D. L., Sheridan, J. (2014). A device to achieve low Reynolds numbers in an open surface water channel. Experiments in Fluids, 55(5), 1-8.

Rawlins, C. B. (1979). Galloping conductors, Chapter 4. EPRI Transmission Line Reference Book: Wind-Induced Conductor Motion.

Raghavan K., (2007). Energy extraction from a steady flow using vortex induced vibration, University of Michigan, Thesis Dissertation.

Raghavan, K., Bernitsas, M. M., Maroulis, D. (2007, July). Effect of Reynolds number on vortex induced vibrations. In Proceedings of the IUTAM Symposium (pp. 23-26).

Rizzoni G., Hartley T.T., (2000). Principles and applications of electrical engineering, Mcgraw Hill.

Sanchez-Sanz M., Fernandez B., Velazquez, A., (2009). Energy-harvesting micro-resonator based on the forces generated by the Karman Street around a rectangular prism, Journal of Microelectromechanical systems 18, 449-457.

Sarpkaya, T. (2004). A critical review of the intrinsic nature of vortex-induced vibrations. Journal of Fluids and Structures, 19(4), 389-447.

Sarpkaya, T. (2010). Wave forces on offshore structures. Cambridge University Press.

Serrano, J. S. (2013). Experimental studies on transitional separated boundary layers.

Seyed-Aghazadeh, B., Modarres-Sadeghi, Y. (2015). An experimental investigation of vortexinduced vibration of a rotating circular cylinder in the crossflow direction. Physics of Fluids (1994present), 27(6), 067101.

Shen, L., Chan, E. S., Lin, P. (2009). Calculation of hydrodynamic forces acting on a submerged moving object using immersed boundary method. Computers and Fluids, 38(3), 691-702.

Shiels, D., Leonard, A., Roshko, A. (2001). Flow-induced vibration of a circular cylinder at limiting structural parameters. Journal of Fluids and Structures, 15(1), 3-21.

Shu, Y. C., Lien, I. C. (2006). Analysis of power output for piezoelectric energy harvesting systems. Smart materials and structures, 15(6), 1499.

Simpson, A. (1972). Determination of the natural frequencies of multiconductor overhead transmission lines. Journal of Sound and Vibration, 20(4), 417-449. 
Singh, S. P., Mittal, S. (2005). Vortex-induced oscillations at low Reynolds numbers: hysteresis and vortex-shedding modes. Journal of Fluids and Structures, 20(8), 1085-1104.

Singh, K., Michelin, S., De Langre, E. (2012). Energy harvesting from axial fluid-elastic instabilities of a cylinder. Journal of Fluids and Structures, 30, 159-172.

Sirohi, J., Mahadik, R. (2011). Piezoelectric wind energy harvester for low-power sensors. Journal of Intelligent Material Systems and Structures, 1045389X11428366.

Sirohi J., Mahadik R., (2012). Harvesting Wind Energy Using a Galloping Piezoelectric Beam, Journal of Vibration and Acoustics 134(1), 011009.

Skop, R. A., Balasubramanian, S. (1997). A new twist on an old model for vortex-excited vibrations. Journal of Fluids and Structures, 11(4), 395-412.

Stansby, P. K., Rainey, R. C. T. (2001). On the orbital response of a rotating cylinder in a current. Journal of Fluid Mechanics, 439, 87-108.

Stephen, N. G. (2006). On energy harvesting from ambient vibration. Journal of sound and vibration, 293(1), 409-425.

Stojković, D., Breuer, M., Durst, F. (2002). Effect of high rotation rates on the laminar flow around a circular cylinder. Physics of Fluids (1994-present), 14(9), 3160-3178.

Tang, L., Païdoussis, M. P., Jiang, J. (2009). Cantilevered flexible plates in axial flow: energy transfer and the concept of flutter-mill. Journal of Sound and Vibration, 326(1), 263-276.

Tang, X., Zuo, L. (2011). Enhanced vibration energy harvesting using dual-mass systems. Journal of Sound and Vibration, 330(21), 5199-5209.

Taylor, G. W., Burns, J. R., Kammann, S. A., Powers, W. B., Welsh, T. R. (2001). The energy harvesting eel: a small subsurface ocean/river power generator. IEEE journal of oceanic engineering, 26(4), 539-547.

Vicente-Ludlam, D., Barrero-Gil, A., Velazquez, A. (2014). Optimal electromagnetic energy extraction from transverse galloping. Journal of Fluids and Structures, 51, 281-291.

Vicente-Ludlam, D., Barrero-Gil, A., Velazquez, A. (2015). Enhanced mechanical energy extraction from transverse galloping using a dual mass system. Journal of Sound and Vibration, 339, 290-303.

Wang, G. Q., Lu, Y. M. (2014). An improved lumped parameter model for a piezoelectric energy harvester in transverse vibration. Shock and Vibration, 2014.

Williamson, C. H. K., Govardhan, R. (2004). Vortex-induced vibrations. Annu. Rev. Fluid Mech., $36,413-455$.

Williamson, C. H. K., Roshko, A. (1988). Vortex formation in the wake of an oscillating cylinder. Journal of fluids and structures, 2(4), 355-381. 
Xu-Xu, J., Barrero-Gil, A., Velazquez, A. (2015). A theoretical study of the coupling between a vortex-induced vibration cylindrical resonator and an electromagnetic energy harvester. Smart Materials and Structures, 24(11), 115009.

Xu-Xu, J., Barrero-Gil, A., Velazquez, A. (2016a). Experimental study on transverse flow-induced oscillations of a square-section cylinder at low mass ratio and low damping. Experimental Thermal and Fluid Science, 74, 286-295.

$\mathrm{Xu}-\mathrm{Xu}$, J., Vicente-Ludlam, D., Barrero-Gil, A. (2016b). Theoretical study of the energy harvesting of a cantilever with attached prism under aeroelastic galloping. European Journal of MechanicsB/Fluids, 60, 189-195.

Yang, Y., Zhao, L., Tang, L. (2013). Comparative study of tip cross-sections for efficient galloping energy harvesting. Applied Physics Letters, 102(6), 064105.

Yu, D., Mei, R., Luo, L. S., Shyy, W. (2003). Viscous flow computations with the method of lattice Boltzmann equation. Progress in Aerospace Sciences, 39(5), 329-367.

Zhao, L., Tang, L., Yang, Y. (2012). Small wind energy harvesting from galloping using piezoelectric materials. In ASME 2012 Conference on Smart Materials, Adaptive Structures and Intelligent Systems (pp. 919-927). American Society of Mechanical Engineers.

Zhao, L., Tang, L., Yang, Y. (2013). Comparison of modeling methods and parametric study for a piezoelectric wind energy harvester. Smart materials and Structures, 22(12), 125003.

Zhao, L., Yang, Y. (2015). Enhanced aeroelastic energy harvesting with a beam stiffener. Smart Materials and Structures, 24(3), 032001.

Zhu, Q., Haase, M., Wu, C. H. (2009). Modeling the capacity of a novel flow-energy harvester. Applied Mathematical Modelling, 33(5), 2207-2217.

Zhu, Q., Peng, Z. (2009). Mode coupling and flow energy harvesting by a flapping foil. Physics of Fluids (1994-present), 21(3), 033601.

Zou, Q., He, X. (1997). On pressure and velocity boundary conditions for the lattice Boltzmann BGK model. Physics of Fluids (1994-present), 9(6), 1591-1598. 